Ecophysiology and environmental distribution of organohalide-respiring bacteria

Yue Lu 


\section{Thesis committee}

\section{Promotor}

Prof. Dr Hauke Smidt

Personal chair at the Laboratory of Microbiology

Wageningen University

\section{Co-promotor}

Dr Siavash Atashgahi

Post-Doc, Laboratory of Microbiology

Wageningen University

\section{Other members}

Prof. Dr Huub Rijnaarts, Wageningen University

Dr Jan Dolfing, Newcastle University, United Kingdom

Dr Jan Gerritse, Deltares, Utrecht, The Netherlands

Dr Ivonne Nijenhuis, Helmholtz Centre for Environmental Research - UFZ, Leipzig, Germany

This research was conducted under the auspices of the Graduate School for Socio-Economic and Natural Sciences of the Environment (SENSE) 


\title{
Ecophysiology and environmental distribution of organohalide-respiring bacteria
}

\author{
Yue Lu
}

Thesis

submitted in fulfillment of the requirement for the degree of doctor at Wageningen University

by the authority of the Rector Magnificus

Prof. Dr A.P.J. Mol, in the presence of the

Thesis Committee appointed by the Academic Board

to be defended in public

on Tuesday 13 September 2016

at 4 p.m. in the Aula. 
Yue $\mathrm{Lu}$

Ecophysiology and environmental distribution of organohaliderespiring bacteria

240 pages.

$\mathrm{PhD}$ thesis, Wageningen University, Wageningen, NL (2016)

With references, with summary in English

ISBN 978-94-6257-841-8

DOI $10.18174 / 384760$ 


\section{Contents}

Chapter 1 General introduction and outline.

Chapter 2 Overview of known organohalide-respiring bacteria - phylogenetic diversity and environmental distribution

Chapter 3 Primers that target functional genes of organohalide-respiring bacteria

Chapter 4 Functional genomics of corrinoid starvation in the organohalide-respiring bacterium Dehalobacter restrictus strain PER-K23

Chapter 5 Dehalogenation of three tetrachlorobenzene isomers by contaminated harbor

sludge-derived enrichment cultures follows thermodynamically favorable reactions

Chapter 6 Geochemical and microbial analysis of groundwater at a site with biostimulated reductive dechlorination

Chapter 7 Organohalide respiration in pristine hypersaline lakes....

Chapter 8 General discussion

References

Summary

Appendices 



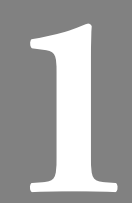

General introduction and outline 


\section{Organohalides}

Organohalides are halogenated organic compounds containing one or more carbon-halogen bonds in their structure. With different halogens, i.e. fluorine, chlorine, bromine and iodine, substituting hydrogen atoms in aliphatic and aromatic hydrocarbons, diverse organohalides are formed and referred to as organofluorine, organochlorine, organobromine and organoiodine compounds.

The organohalides found in the environment are of natural as well as anthropogenic origin. Natural sources include geogenic processes (forest fires, meteorites, volcanoes and other geothermal activities) and biosynthesis of organohalides by a broad range of different (micro)organisms, plants and animals $[1,2]$. Additionally, enormous quantities of organohalides have been produced via anthropogenic activities [3]. Due to their extensive structural, chemical and physical variety and desirable industrial properties, organohalides have been manufactured for a wide range of industrial and agricultural applications, including fire extinguishants, solvents used e.g. for dry cleaning and degreasing of metal surfaces, refrigerants, pesticides and pharmaceutical agents [3]. As a result of massive use and uncontrolled disposal on one hand and high persistence and bioaccumulation in ecosystems on the other hand, organohalides have become problematic environmental chemicals [4], and some of them are proven to be toxic and threaten the health of humans and other animals [5].

\section{Reductive dehalogenation}

To clean up organohalide pollutants in the environment, many efforts have been made [6]. However, organohalides are found recalcitrant to biodegradation, which is mainly due to a combined influence of site biogeochemical parameters and the lack of (the activity of) degrading microbes [7]. Especially highly halogenated compounds are hardly mineralized under oxic conditions, leading to their (bio)accumulation [8,9]. For their biotransformation and/or detoxification under anoxic conditions, reductive dehalogenation, also termed as organohalide-respiration (OHR) [7], is the only documented microbial process [6] and has been observed in many oxygen-depleted environments such as subsurface soil, wastewater treatment reactors, groundwater, aquifers and marine sediments. During OHR, organohalides are utilized as terminal electron acceptors for anaerobic respiration. Because organohaliderespiring bacteria (OHRB) use organohalides in their energy metabolism, OHR is considered as a feasible process to produce less-halogenated metabolites that can ultimately lead to the complete detoxification of organohalides and/or render them susceptible to further 
downstream (bio)transformations, and therefore contribute to alleviating the environmental problem of organohalide pollutants [10].

\section{Organohalide-respiring bacteria (OHRB)}

OHRB are able to use organohalides as terminal electron acceptors and couple OHR to energy conservation. Since the first organohalide-respiring isolate, Desulfomonile tiedjei DCB1 [11], that was characterized as a 3-chlorobenzoate respirer, a large number of OHRB have been isolated/described. OHRB isolates are reported to utilize various organohalides for growth, including (but not limited to) trichloromethane, trichloroethane, tetra- and tri-chloroethene, chlorinated/brominated phenols and benzenes, dioxins, polychlorinated biphenyls and polybrominated diphenyl ethers [12]. Therefore, these organisms have received considerable scientific/applied interests due to their only recently discovered mode of anaerobic respiration as well as their potential application in organohalide bioremediation [12, 13]. A good example is the acceleration of in situ reductive dehalogenation of chlorinated ethenes by adding electron donors and/or exogenous OHRB containing enrichment cultures via biostimulation and bioaugmentation, respectively [14]. However, the known OHRB are fastidious microbes, and their efficient application in organohalide bioremediation has been held back by lack of thorough understanding of metabolic capabilities, optimized growth conditions, dependency on other microbial guilds for their nutritional requirements as well as interactions with physical chemical parameters and their environmental distribution. To fill this knowledge gap, this dissertation focuses on enhancing our understanding of the growth and physiology of OHRB, improving our knowledge of dehalogenation activity and interactions of OHRB with non-dechlorinating microbes as well as the affecting physical chemical parameters at bench and field scales, and exploring their environmental distribution in contaminated and pristine sites. To this end, a combination of analytical chemistry, physiological approaches and molecular techniques including quantitative PCR, next generation sequencing, transcriptional analysis and shotgun proteomics are applied (Figure 1.1).

\section{Molecular approaches applied to study OHRB and their reductive dehalogenase (RDase) genes}

To investigate potential OHRB in the studies described in this thesis, culture-independent methods based on 16S ribosomal RNA (rRNA) gene amplicon sequencing and quantitative PCR (qPCR) that target phylogenetic markers of OHRB and their genes encoding RDases, the key enzymes catalyzing OHR, are applied. qPCR is a powerful diagnostic technique that has been applied as a fast and sensitive strategy for identifying and monitoring OHRB and RDase 


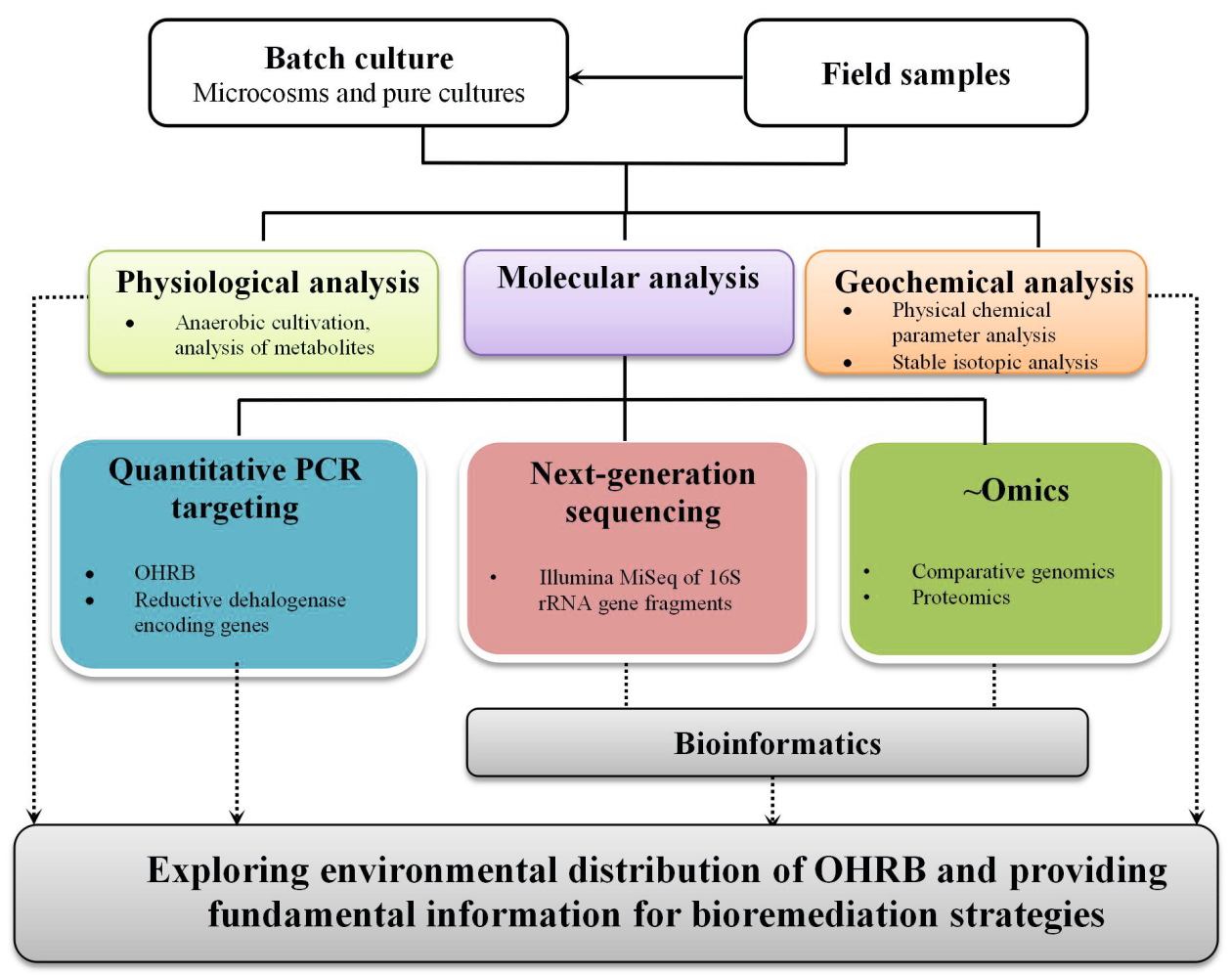

Figure 1.1 Approaches used in this thesis.

genes involved in the biotransformation of organohalides, and it has been wildly used to guide bioremediation efforts [15]. In this study, a series of qPCR assays are applied to target $16 \mathrm{~S}$ rRNA and RDase encoding genes of OHRB to quantify their absolute or relative abundance. To explore broader bacterial community composition based on 16S rRNA gene, highthroughput sequencing is conducted on the Illumina MiSeq platform. Compared to the lowresolution molecular methods that generate a fingerprint of main microbial community members, e.g. a combination of terminal restriction fragment length polymorphism (T-RFLP) or denaturing gradient gel electrophoresis (DGGE) with cloning and Sanger sequencing, next generation technology sequencing platforms such as those developed by Illumina (MiSeq, HiSeq) can provide vast quantities of data and the cost per sequence is much lower $[16,17]$. On the downside, these new sequencing technologies also have their limitations like generally shorter read length and higher error rates than Sanger sequencing [17-19].

To further explore the metabolism of OHRB isolates, omics techniques, including comparative genomics and proteomics analyses, are applied in this thesis. With the increasing 
number of released genomes of OHRB, comparative genomics studies provide the necessary basis for comprehensive understanding of their physiology, metabolism and regulation mechanisms [20-22]. Furthermore, these approaches offer insights into the mechanisms of evolution and can reveal patterns/evidence of acquisition of e.g. RDase encoding genes via horizontal gene transfer [23]. In our study, comparative genomics is applied to investigate the organization of corrinoid operons in six available genomes of Dehalobacter spp. to better understand the corrinoid auxotrophy of Dehalobacter restrictus. Additionally, highthroughput proteomics has been applied in many studies of OHRB isolates [24-29], which aids in functional characterization of OHRB strains at the protein level, and can expand our knowledge of their potential substrate spectrum and regulation of RDases, the electron transport mechanism of OHR, and the metabolism and activity of OHRB. In our study, shotgun proteomics is applied to elucidate the response of $D$. restrictus under partial corrinoid starved conditions and to reveal its corrinoid metabolism and regulation mechanism at the protein level.

\section{Stable isotopic analyses to study OHR(B)}

Stable isotopic analysis has been widely used in the study of OHR(B) at both field and laboratory scales to i) distinguish abiotic and biotic transformation of organohalides [30-33], ii) identify the specific stable isotope fractionation caused by different OHRB and define reaction mechanisms $[30,31,34,35]$, iii) reveal the factors (e.g. growth substrates, transport across membranes, binding to the enzyme) that may control the stable isotope fractionation of different organohalides during OHR [31], iv) and explore the central metabolism [36], such as study the Wood-Ljungdahl pathway in Dehalococcoides mccartyi [37]. Stable isotopic analysis, and in particular the high resolution dual carbon-chlorine isotopic analysis, has been applied as a useful complementary tool to evaluate (bio)remediation of organohalide pollutants [33, 34, 38]. In our study, carbon isotopic signatures of cis-dichloroethene (DCE) and vinyl chloride are investigated during biostimulation of a cis-DCE-contaminated aquifer and applied as an informative line of evidence to build up a conceptual model of metabolic interactions within microbial communities.

\section{Thesis outline}

This dissertation has two sections, i.e. Section one with two review chapters (Chapter 2-3) and Section two with four research chapters (Chapter 4-7). In Chapter 2, phylogeny, genetic characteristics and ecophysiology of the different OHRB known to date are described. Further, important factors impacting the ecology and environmental distribution of OHRB are 
discussed in this chapter. Chapter 3 provides an overview of the primers targeting functional genes of OHRB. Primers that have been developed to target reductive dehalogenase-encoding genes are summarized and their application in the characterization and quantification of OHRB and reductive dehalogenase homologous genes are described in this chapter.

Chapter 4 aims at exploring the impact of corrinoid starvation on Dehalobacter restrictus strain PER-K23, which is restricted to OHR for energy conservation. D. restrictus is known for its corrinoid auxotrophy, and the strategy it develops to satisfy this key nutritional need is studied by combining physiological and molecular techniques including comparative genomics, transcriptional analysis and proteomics.

Chapter 5 reports dechlorination of three tetrachlorobenzene (TeCB) isomers by anoxic microcosms derived from contaminated harbor sludge. The thermodynamically preferred biotransformation pathways of $\mathrm{TeCB}$ isomers are investigated, and molecular tools are utilized to identify microorganisms responsible for $\mathrm{TeCB}$ dechlorination. The aim is to broaden current knowledge of the under-explored non-dechlorination community members involved in chlorobenzene dechlorination and their potential relationship with dechlorinators. Increased data availability on the key microbial players is a priority to boost the applicability of bioremediation.

Chapter 6 reports biostimulation with glycerol to enhance reductive dechlorination in a cisDCE-polluted aquifer and monitoring of geochemical and microbial evolution to reveal subsurface responses to biostimulation. The results identify some common but also some previously overlooked microbial guilds that, intertwined with site geochemical and hydrological parameters, can promote/prohibit enhanced reductive dechlorination.

The research described in Chapter 7 seeks OHR potential from extreme pristine environments, and more specifically in hypersaline lake sediments collected from Western Australia that have recently been reported as sources of biological chloromethane and chloroform emission [39]. The OHR potential is evaluated using microcosms prepared from hypersaline sediment, and microbial community analysis in combination with quantification of the known OHRB and reductive dehalogenase genes are applied. This extends OHR to extreme pristine environments and provides important implications for a local chlorine cycle between biotic chlorinators and dechlorinators. 
Chapter 8 provides a general discussion of the results presented in this dissertation, and concludes with an outlook for future perspectives in the study of ecophysiology and environmental distribution of OHRB, the physical and chemical parameters affecting OHR(B), the interactions of OHRB with other microbial guilds in the community, and the application of this fundamental information for future bioremediation strategies. 


\section{Overview of known organohalide-}

respiring bacteria - phylogenetic diversity

and environmental distribution

A modified version of this chapter has been accepted for publication:

Siavash Atashgahi\#, Yue Lu\# and Hauke Smidt. 2016.

Overview of known organohalide- respiring bacteria - phylogenetic diversity and environmental distribution. In: Organohalidem- Respiring Bacteria. Springer. 


\begin{abstract}
To date, organohalide-respiration (OHR) has been restricted to the bacterial domain of life. Known organohalide-respiring bacteria (OHRB) are spread among several phyla comprising both Gram-positive and Gram-negative bacteria. As a unique trait, OHRB benefit from reductive dehalogenase enzymes enabling them to use different organohalides as terminal electron acceptors and occupy a wide range of terrestrial and aquatic environments. This chapter comprises three sections: Firstly, we give an overview of phylogeny of known OHRB and briefly discuss physiological and genetic characteristics of each group. Secondly, the environmental distribution of OHRB is presented. Owing to the application of molecular diagnostic approaches, OHRB are being increasingly detected not only from organohalidecontaminated groundwaters and sediments but also from pristine environments, including deep oceanic sediments and soils that are ample sources of naturally-occurring organohalides. Finally, we highlight important factors that impact the ecology of OHRB and their interaction with other microbial guilds.
\end{abstract}




\section{Introduction}

The vast functional diversity of microorganisms and their metabolic capabilities have made them successful mediators of electron liberation from the oxidation of inorganic and organic matter coupled to the reduction of a wide array of inorganic and organic electron acceptors including organohalides [7]. Although most well-known representatives of organohalides are considered to be man-made products of industrial origin, thousands of naturally occurring organohalides have been reported from geogenic and biogenic sources [2]. Numerous reports exist on natural production of organohalide compounds from biogenic sources such as a broad range of seaweeds, sponges, terrestrial plants, fungi, as well as through geogenic processes such as volcanic activity, forest fires, and other geothermal processes, some of which predate the industrialization era. Such ancient natural production of organohalides might have contributed to the development of biochemical strategies capable of unlocking the chemically stable carbon-halogen bond in organohalides. This is particularly important for nonoxygenolytic dehalogenation processes that probably have developed in the originally oxygen-free atmosphere on Earth. Taking advantage of organohalides as thermodynamically favourable electron acceptors under anoxic conditions, reductive dehalogenation is used as a terminal electron accepting process by organohalide-respiring bacteria (OHRB). These microbes have greatly contributed to global cycling of halogens by breathing (rather) toxic organohalides and preventing their accumulation in the environment. Exploiting reductive dehalogenases (RDase in case of functionally characterized enzymes, and RdhA for yet uncharacterized reductive dehalogenases predicted from genomes and molecular surveys) dedicated to organohalide respiration (OHR), OHRB occupy a wide range of niches/environments. Hence, understanding factors governing their evolution, distribution and ecology will help to unravel their role in the fate of organohalides. It should be noted that organohalide degradation processes have been described for a wide range of redox conditions (from highly oxidising to strictly reducing), and mediated by a wide variety of (micro)organisms in co-metabolic and/or energy-yielding modes, however, in this chapter we specifically discuss the phylogeny and environmental distribution of OHRB that are known to gain energy and grow using organohalides as electron acceptors.

\section{Phylogeny of OHRB}

Since the description of Desulfomonile tiedjei as the first isolated OHRB [11], numerous bacterial strains capable of OHR have been obtained in axenic culture (Figure 2.1), providing 


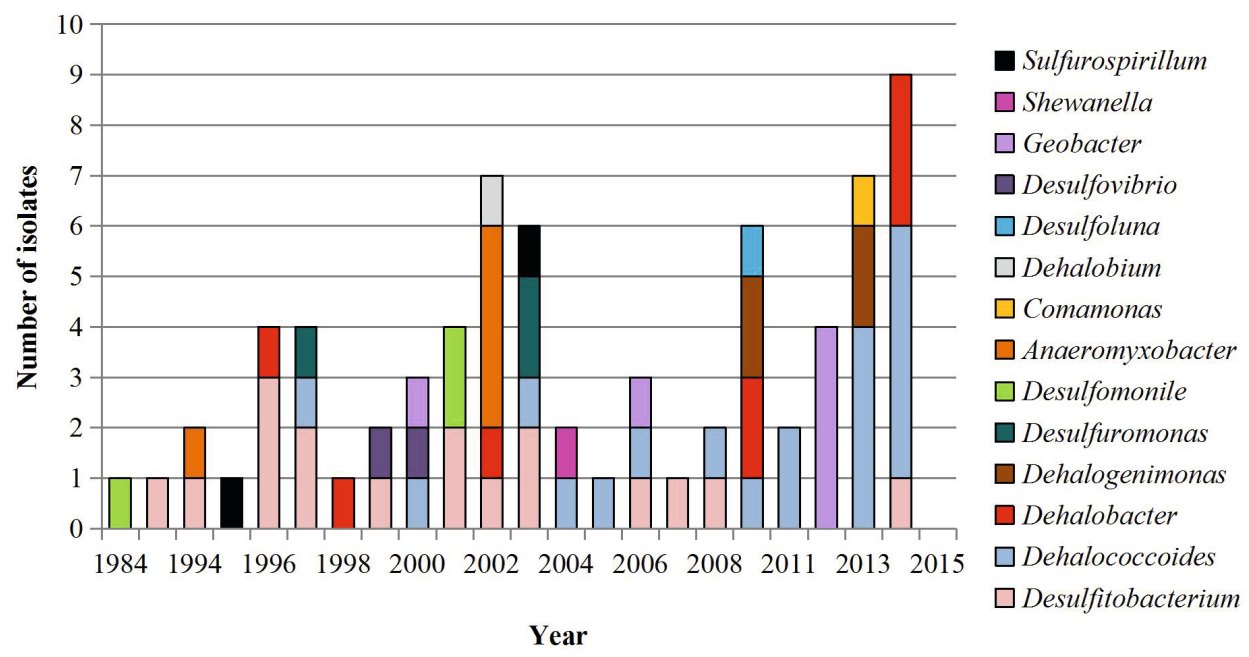

Figure 2.1 Number of OHRB available in axenic culture. The data is based on the number of isolates as of July 2015.

indispensable insights into their phylogenetic, physiological, and biochemical traits. Members of the genus Dehalococcoides comprise the biggest groups of isolates to date (19 isolates) followed by strains of Desulfitobacterium (17 isolates) (Figure 2.1). The known OHRB arespread among several distinct phyla comprising both Gram-positive and Gram-negative bacteria (Figure 2.2). The known isolates can by and large be divided into facultative and obligate groups based on whether OHR is their only energy-gaining metabolism [13]. The members of the facultative OHRB are characterized by a more versatile metabolism, in general have the ability to grow on a wide range of electron acceptors, and include proteobacterial OHRB such as Geobacter, Desulfuromonas, Anaeromyxobacter, Desulfomonile, Desulfovibrio, Desulfoluna, Sulfurospirillum, Comamonas, Shewanella as well as Desulfitobacterium from the phylum Firmicutes. The fact that some facultative OHRB such as Comamonas, Geobacter and Shewanella belong to phylogenetic groups that mostly comprise non - OHRB points towards horizontal acquisition of reductive dehalogenase genes. The obligate OHRB on the other hand are restricted to OHR for energy conservation and growth and include Dehalobacter (phylum Firmicutes) and the OHRB belonging to the Dehalococcoidia class (phylum Chloroflexi) including strains of Dehalococcoides mccartyi, Dehalogenimonas spp. and the single isolate 'Dehalobium chlorocoercia' DF-1[13, 40, 41]. However, recent studies showed fermentative growth of Dehalobacter spp. on chloromethane 
[42, 43], suggesting that at least some of the isolates previously considered obligate OHRB might harbour additional modes of metabolism beyond the canonical OHR. It is also interesting to note that recent single cell genomic studies of marine Dehalococcoidia did not reveal any evidence for catabolic reductive dehalogenation, indicating that microorganisms closely related to known obligate OHRB do not rely on OHR for energy conservation, but rather utilize organic matter degradation pathways [44, 45].

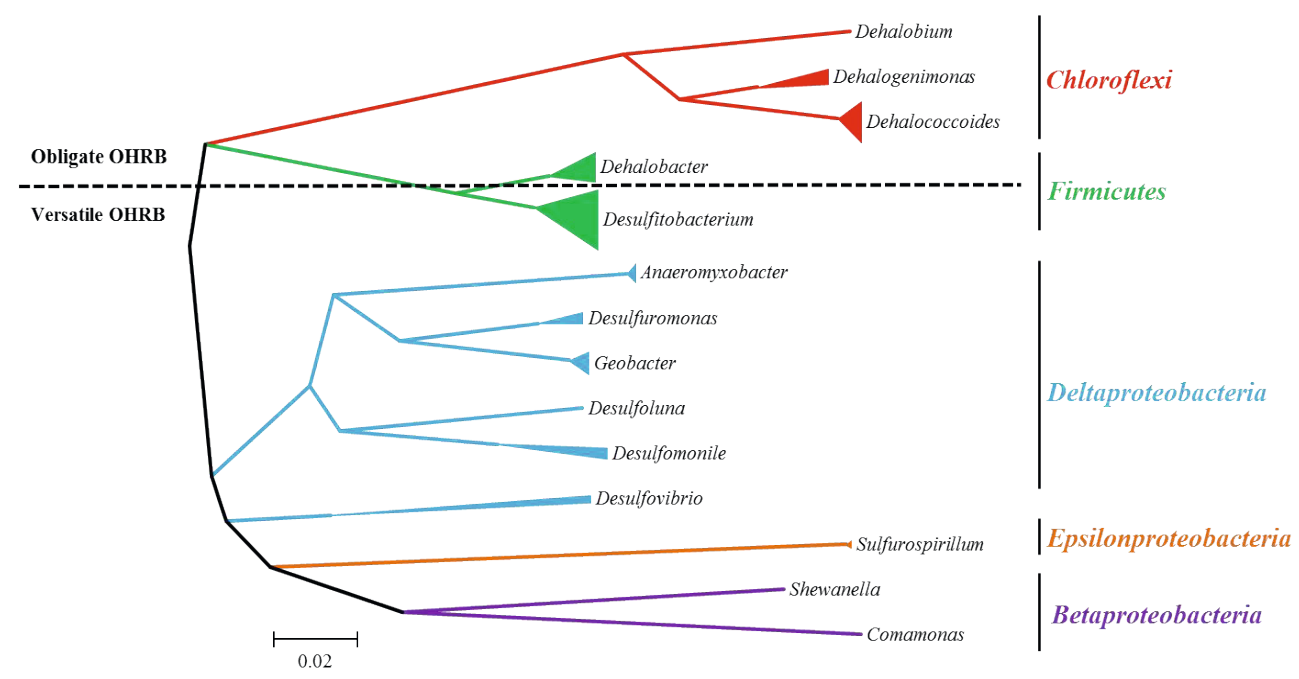

Figure 2.2 Phylogenetic tree of known OHRB based on bacterial 16S rRNA gene sequences. Alignment and phylogenetic analysis were performed with MEGA and the tree was constructed using the neighbour-joining (NJ) method. The reference bar at the bottom indicates the branch length that represents $2 \%$ sequence divergence. Colour Key: Chloroflexi (red), Firmicutes (green), Deltaproteobacteria (blue), Betaproteobacteria (violet), Epsilonproteobacteria (brown).

With few exceptions, OHRB from different phyla, i.e. members of the Firmicutes, Chloroflexi and different classes of Proteobacteria, benefit from similar enzymes/pathways for OHR indicating that these genes could be acquired from common ancestors via transposon mediated dissemination. However, to date little to no correlation could be found between the type of organohalides used as electron acceptor and the phylogenetic affiliation of OHRB, and haloaliphatic and haloaromatic compounds can be dehalogenated by isolates of taxonomically different genera [46]. Furthermore, isolates with similar phylogeny and physiology have been obtained from very different environments. For example, Desulfuromonas michiganensis strain BB1 was isolated from pristine river sediment while the closely related strain BRS1 was obtained from chloroethene-contaminated aquifer material [47]. Similarly, the closely 
related uncultured Chloroflexi Lahn and Tidal Flat Clusters were both capable of dechlorination of perchloroethene (PCE) to trans-DCE while being enriched from sediments from river and marine environments, respectively [48, 49]. Another example is the case of Dehalococcoides mccartyi strains KS and RC. Both bacteria grow with 1,2-dichloropropane (1,2-D) as an electron acceptor in enrichment cultures while being derived from hydrocarboncontaminated and pristine river sediments, respectively $[50,51]$.

In the following, we will separately discuss OHRB from different phyla.

\section{Firmicutes}

\section{Dehalobacter}

Dehalobacter has been first described as a genus in 1998, belonging to the Firmicutes (Phylum), Clostridia (Class), Clostridiales (Order) and Peptococcaceae (Family) [52]. Dehalobacter restrictus PER-K23 ${ }^{\mathrm{T}}$, the first isolate and the type species of this genus, was obtained from an anaerobic PCE-dechlorinating packed-bed column, and was found to be able to couple the reductive dehalogenation of PCE to growth and energy conversation $[52,53]$. Subsequently, additional pure cultures were isolated and characterized from this genus (Figure 2.1) [54-58]. Dehalobacter spp. are known as obligate OHRB with relatively small genomes $(2.60 \mathrm{Mb}-3.09 \mathrm{Mb}$, with exception of Dehalobacter sp. FTH1 with a genome size of $6.33 \mathrm{Mb}$ ), and are restricted to organohalide respiration with $\mathrm{H}_{2}$ as sole electron donor except for two reports on fermentative growth of Dehalobacter spp. with chloromethane [42, 43]. The presence of up to 39 copies of putative RDH catalytic subunit encoding $r d h A$ genes in currently available genomes indicates their potentially broader substrate spectrum. Cultivation and environmental studies also revealed an increasing number of halogenated compounds that can be used by Dehalobacter spp. as electron acceptors including PCE and trichloroethene (TCE) [53, 54, 58], chlorinated ethanes [56, 59], 2,4,6-trichlorophenol $(2,4,6-$ TCP) [58], 2,4,6-tribromophenol (2,4,6-TBP) [60], chloroform [61], chlorinated benzenes [57, 62], $\beta$-hexachlorocyclohexane $(\beta-\mathrm{HCH}$, known as lindane) [63], and phthalide $(4,5,6,7-$ tetrachlorophthalide) [55]. One RDase (PceA) was functionally characterized from Dehalobacter spp. [64], which displayed a corrinoid as essential cofactor. Recent studies showed that most Dehalobacter spp. genomes have a complete anaerobic cobalamin biosynthesis pathway [65], however, no Dehalobacter spp. have been confirmed to biosynthesize cobalamin de novo $[52,55,56,63]$. Salvaging cobinamide from the environment, e.g., from cobalamin-producing non-dehalogenating community members such 
as Sedimentibacter spp. [63, 66] was suggested as the strategy Dehalobacter spp. employ to ensure corrinoids supply for growth and reductive dechlorination [65, 67].

\section{Desulfitobacterium}

Desulfitobacterium is a genus belonging to the Firmicutes (Phylum), Clostridia (Class), Clostridiales (Order) and Peptococcaceae (Family). Desulfitobacterium spp. isolates are among the best characterized facultative OHRB that are known for their versatile metabolism with the capability to use a wide variety of electron donors and acceptors [68]. The first OHRB isolated in this genus was originally related to the genus Clostridium [69]. Strain DCB-2, isolated from municipal sludge, is a Gram-positive, strictly anaerobic, ortho- and meta-chlorophenol-dechlorinating bacterium [69]. Two years later, with the isolation of the monotypic strain Desulfitobacterium dehalogenans JW/IU-DC1 ${ }^{\mathrm{T}}$, the Desulfitobacterium genus was proposed as a new taxon [70]. This genus was designated Desulfitobacterium because strain JW/IU-DC1 ${ }^{\mathrm{T}}$ grows with sulfite, but not with sulfate, as a terminal electron acceptor [70]. This was followed by re-classification of strain DCB-2 as the type strain of Desulfitobacterium hafniense [71]. The species Desulfitobacterium frappieri was proposed with the isolation of strain PCP-1, an anaerobic pentachlorophenol-dechlorinating bacterium isolated from a methanogenic consortium [72]. Based on DNA-DNA hybridization and comparative physiological studies $D$. hafniense, D. frappieri, and the non-OHRB strain GBFH were later united into the species D. hafniense [73]. Other OHRB belonging to the genus Desulfitobacterium isolated in the following years are indicted in Table 2.1.

Completed [20, 74, 75] and ongoing (Kruse et al., in preparation) genome sequencing projects have identified 1-7 copies of RDH encoding genes in the genomes of Desulfitobacterium spp., far less than the closely related dehalogenating clade of Dehalobacter spp. that contain up to 39 copies [23]. The loss of the OHR trait upon growth on alternative electron acceptors further confirmed the physiological opportunism of organohalide-respiring strains of Desulfitobacterium spp., and it was proposed that the RDase genes were acquired by horizontal gene transfer from closely related obligate organohalide-respiring strains of Dehalobacter spp. [76]. Nevertheless, Desulfitobacterium spp. strains are of high interest in the OHR field due to their high growth rate and respiration with a diverse range of organohalides, though none of the isolates were able to dechlorinate PCE past cis-DCE. Diverse RDases were purified and characterized from Desulfitobacterium spp. including chlorophenol RDase [77, 78], PCE RDase [79-82] and dichloroethane (DCA) RDase [83]. 
Additionally, a complete de novo corrinoid synthesis pathway was identified from the genome of D. hafniense Y51 [74].

\section{Chloroflexi}

\section{Dehalococcoides}

Dehalococcoides bacteria belong to Chloroflexi (Phylum), Dehalococcoidia (Class), Dehalococcoidales (Order) and Dehalococcoidaceae (Family) [40], which are well known for their metabolic restriction to OHR. The genus was first established in 1997 with the isolation of D. ethenogenes $195^{\mathrm{T}}$ capable of complete dechlorination of PCE to ethene [84]. In following years, additional isolates of this genus were obtained in axenic culture including CBDB1, BAV1, VS, FL2 and GT strains. These five strains and the type strain $D$. ethenogenes 195 were re-classified as the new genus and species, Dehalococcoides mccartyi [40]. Recently, more strains from this species were isolated including strain JNA [85, 86], CG1 [87, 88], CG4 [87, 88], CG5 [87, 88], SG1 [87, 88], AD14-1 [89] and AD14-2 [89]. Based on an earlier 16S rRNA gene-based classification, Hendrickson, Payne [90] divided isolates and clones available at the time into three phylogenetic subgroups, a classification which has been widely referred to ever since. Accordingly, D. ethenogenes $195^{\mathrm{T}}$ belongs to the 'Cornell' subgroup, strain VS belongs to the 'Victoria' subgroup, and subgroup 'Pinellas' contains most of the cultured strains such as CBDB1, BAV1, FL2 and GT and the majority of environmental sequences. Common traits of D. mccartyi strains are streamlined genomes, small and non-motile coccoid cells, resistant to penicillin-type antibiotics, obligately anaerobic, and restricted to OHR as the only mode of metabolism using $\mathrm{H}_{2}$ as the only electron donor [40]. Similar to the situation described above for Dehalobacter spp., genomic studies of known isolates showed the lack of de novo vitamin B12 synthesis pathways [91, 92] although they are capable of corrinoid salvaging and remodelling from their growth medium [93-95].

In spite of sharing more than $98 \%$ identity with respect to their $16 \mathrm{~S}$ rRNA gene sequences, different strains respire on different ranges of organohalides. For example, vinyl chloride (VC) respiration is limited to strains BAV1, GT and VS while none of them can respire on PCE. This might be due to large diversity of $r d h A$ genes that are present in multiple non-identical copies on individual genomes. However, in spite of multiple homologous RDH genes the number of which currently ranges from 11 copies in strain BAV1 up to 36 copies in strain VS [96], only a limited number of RDases was purified and characterized from D. mccartyi 
strains including PCE RDase [97], TCE RDase [98], trans-DCE producing RDase [99], vinyl chloride RDase [100-102], chlorobenzene RDase [103, 104], dichloropropane RDase [104] and polychlorinated biphenyls (PCBs) RDase [88].

\section{Dehalogenimonas}

The genus Dehalogenimonas belongs to the Chloroflexi (Phylum), Dehalococcoidia (Class), Dehalococcoidales (Order) and Dehalococcoidaceae (Family) [40]. Up to now, two species were described that were isolated from groundwater contaminated with chlorinated alkanes and alkenes. Dehalogenimonas lykanthroporepellens was proposed as a new species in 2009 [105], with the isolation of two strains BL-DC-9T and BL-DC-8, both Gram-negative, nonmotile, non-spore forming, polychlorinated aliphatic alkanes-dechlorinating bacteria [106]. The genome of D. lykanthroporepellens BL-DC-9 has been released recently [107], and multiple $r d h A$ genes were found simultaneously transcribed during OHR with different organohalides as the electron acceptors [108]. D. lykanthroporepellens strains show 10-11\% 16S rRNA gene sequence divergence compared to Dehalococcoides [108], perform dihaloelimination and have a higher tolerance to oxygen exposure [106]. The second species, D. alkenigignens, was proposed in 2013 [109] with the isolation of two strictly anaerobic strains IP $3-3^{\mathrm{T}}$ and SBP-1. Both species (D. alkenigignens and D. lykanthroporepellens) have similar morphological features and substrate spectra with $96.2 \%$ pairwise identity of their $16 \mathrm{~S}$ rRNA gene sequence [109]. 1,2-DCA, 1,2-DCP, 1,2,3-DCP, 1,1,2-TCA and 1,1,2,2-TeCA can serve as electron acceptor with $\mathrm{H}_{2}$ as sole electron donor [105, 106, 109].

\section{Dehalobium}

Next to Dehalogenimonas and Dehalococcoides, the class Dehalococcoidia also contains the genus Dehalobium [40] represented by a single isolate informally named 'Dehalobium chlorocoercia' strain DF-1. This bacterium was highly enriched in a PCB-dechlorinating coculture with a sulfate-reducing Desulfovibrio sp. from estuarine sediment of Charleston Harbor, South Carolina [110, 111]. Dehalobium chlorocoercia DF-1 selectively dechlorinates PCB congeners with double-flanked chlorines with formate or $\mathrm{H}_{2}-\mathrm{CO}_{2}$ as the electron donor and carbon source $[110,111]$. Strain DF-1 is the only reported representative of the genus Dehalobium and the classification as 'Dehalobium chlorocoercia' was first proposed in 2008 [48], however, the current genus and species names have yet to be validated. D. chlorocoercia DF-1 shares $89 \% 16 \mathrm{~S}$ rRNA sequence similarity to the ortho-PCB-dechlorinating bacterium $o$-17 [112] while having $\sim 87.5 \%$ similarity with Dehalococcoides[40]. Unlike $o-17$ and $D$. mccartyi strains, strain DF-1 uses formate as the electron donor, however, it requires 
Desulfovibrio spp. in co-culture or its cell extract for growth with hydrogen and PCB in mineral medium [41]. Other phylotypes phylogenetically related to the D. chlorocoercia DF1/ Bacterium o-17 clade within the Chloroflexi were reported to be able to dechlorinate PCB congeners $[113,114]$. In addition to PCB, D. chlorocoercia DF-1 dechlorinates highly chlorinated benzenes [115] and PCE and TCE producing higher amounts of trans-DCE than cis-DCE compared with canonical PCE/TCE dechlorinators [116]. Based on its unique dechlorinating activity, DF-1 has shown merits in PCBs bioaugmentation in contaminated soil [117] and sediment [118].

\section{Deltaproteobacteria}

Anaeromyxobacter

Anaeromyxobacter dehalogenans belongs to the Proteobacteria (Phylum), Deltaproteobacteria (Class), Myxococcales (Order) and Myxococcaceae (Family). Anaeromyxobacter was established as a genus with the monotypic species Anaeromyxobacter dehalogenans $2 \mathrm{CP}-1^{\mathrm{T}}$ to describe a myxobacterium that can grow without air [119]. This bacterium is a Gram-negative, motile by gliding, spore-forming, bright red pigmentsproducing slime rod that is capable of facultative anaerobic growth, and was isolated from stream sediment near Lansing, MI, USA [120]. Subsequently, another four isolates were obtained from different sources including pond sediment, Cameroon rain forest soil and yard compost with acetate and 2-chlorophenol (2-CP) as electron donor and acceptor, respectively [119]. Halophenols such as 2-CP, 2,6-dichlorophenol (2,6-DCP), 2,5-dichlorophenol (2,5DCP), 2-bromophenol (2-BP) as well as nitrate, fumarate and oxygen can serve as electron acceptors with acetate, $\mathrm{H}_{2}$, succinate, pyruvate, formate, and lactate as electron donors [119, 120]. A. dehalogenans is able to grow microaerophilically $[119,120]$, and the genome analysis of $A$. dehalogenans $2 \mathrm{CP}-\mathrm{C}$ showed that it harboured genotypic characteristics of obligately aerobic myxobacteria as well as anaerobic Deltaproteobacteria [121].

\section{Desulfuromonas}

Desulfuromonas, a genus the name of which refers to an elemental sulfur-reducing bacterium, belongs to the Proteobacteria (Phylum), Deltaproteobacteria (Class), Desulfuromonadales (Order) and Desulfuromonadaceae (Family). To date, two species of Desulfuromonas capable of chloroethenes respiration have been reported [47, 122]. Desulfuromonas chloroethenica strain TT4B was isolated from chloroethene-contaminated freshwater sediments in eastern Massachusetts, USA $[122,123]$. Strain TT4B uses PCE, TCE as well as fumarate, polysulfide 
and $\mathrm{Fe}(\mathrm{III})$ nitriloacetate as electron acceptor with acetate or pyruvate as electron donors [122]. The second species, Desulfuromonas michiganensis, currently comprises two strains, $\mathrm{BB} 1$ and BRS1, which were isolated from pristine river sediment and contaminated aquifer materials, respectively, both collected from the state of Michigan, USA, as indicated in the species name [47]. Compared to D. chloroethenica, strains BB1 and BRS1 use a wider range of electron donors and can grow by fermentation on fumarate or malate with succinate as major end product [47]. Besides, they showed higher growth yield and PCE tolerance [47].

\section{Geobacter}

Geobacter is a genus belonging to the Proteobacteria (Phylum), Deltaproteobacteria (Class), Desulfuromonadales (Order) and Geobacteraceae (Family), which is well-studied for its capabilities to oxidize organic compounds coupled to the reduction of insoluble metal oxides [124]. This genus was established with isolation of the non-OHRB Geobacter metallireducens [125]. The first OHRB characterized in this genus was Geobacter thiogenes strain K1 (formerly described as Trichlorobacter thiogenes), an anaerobic trichloroacetatedechlorinating, sulfur-producing, fast-growing (doubling time $=6 \mathrm{~h}$ ) bacterium isolated from contaminated soil [126]. Acetate and acetoin were used as electron donor for reductive dechlorination of trichloroacetate, and fumarate served as alternative electron acceptor [126]. Subsequently, Geobacter lovleyi was described as a novel species with strain SZ as type strain, isolated from non-contaminated freshwater sediment collected from Su-Zi Creek near Seoul, South Korea [127]. This bacterium couples acetate oxidation to PCE dechlorination with cisDCE as the end product for growth. Strain SZ can use pyruvate and $\mathrm{H}_{2}$ as electron donors with a wide range of electron acceptors including PCE, TCE, Fe(III), Mn(IV), U(VI), malate, nitrate, fumarate and elemental sulfur, and no fermentation metabolism was found [127]. Additionally, 16S rRNA gene amplicons derived from G. lovleyi strain SZ-like organisms were found in uranium- and chlorinated compounds-polluted environmental samples by using strain SZ-specific primers. Recent genomic analysis of strain SZ identified the presence of a $77 \mathrm{kbp}$ plasmid, pSZ77, encoding 15 out of the 24 genes required for de novo cobalamin biosynthesis [128]. Another OHRB belonging to the Geobacter genus is G. lovleyi strain KB1, a PCE-to-cis-DCE-dechlorinating bacterium identified in the PCE to ethene dechlorinating consortium KB-1 [129]. More recently, four G. lovleyi isolates Geo7.1, Geo7.2, Geo7.3, and Geo7.4 were obtained from PCE to cis-DCE dechlorinating microcosms established with soil from Fort Lewis, WA, that share $99-100 \%$ nucleotide identity with strain SZ based on $16 \mathrm{~S}$ rRNA gene sequences [128]. 


\section{Desulfoluna}

The genus Desulfoluna belongs to the Proteobacteria (Phylum), Deltaproteobacteria (Class), Desulfobacterales (Order) and Desulfobacteraceae (Family) [130]. Desulfoluna spongiiphila strain $\mathrm{AA} 1^{\mathrm{T}}$, an anaerobic Gram-negative bromophenol- dehalogenating bacterium, was isolated from the marine sponge Aplysina aerophoba collected from the Mediterranean Sea [131], which is so far the only isolate of the genus Desulfoluna with confirmed ability to grow via $\mathrm{OHR}$ and the only pure OHRB culture isolated from sponge to date (Figure 2.4). To enrich and isolate strain $\mathrm{AAl}^{\mathrm{T}}$, lactate was used as electron donor and carbon source, and 2BP served as sole electron acceptor [131, 132]. Diverse halogenated compounds can be completely dehalogenated by strain $\mathrm{AA} 1^{\mathrm{T}}$ including 2-, 3- and 4-BP, 1,4- and 2,6- 2,6-DBP, 2,4,6-tribromophenol (2,4,5,TBP), 2- and 3-iodophenol, 2-bromo-4-fluorophenol and 3,5dibromo-4-hydroxybenzoate, but not monochlorinated or fluorinated phenols. $\mathrm{NaCl}$ was found essential for growth and no fermentation was observed with lactate, pyruvate, fumarate or malate as carbon source [131].

\section{Desulfomonile}

The genus Desulfomonile belongs to the Proteobacteria (Phylum), Deltaproteobacteria (Class), Syntrophobacterales (Order) and Syntrohaceae (Family) [11]. Two species of Desulfomonile were characterized as strictly anaerobic, 3-chlorobenzoate dechlorinating, sulfidogenic bacteria with special 'collar' girdling cells [11, 133]. Desulfomonile tiedjei was proposed as a novel and type species in 1990 [11] with strain DCB- ${ }^{\mathrm{T}}$, a Gram-negative, nonmotile, non-spore-forming, anaerobic rod-shaped bacterium, which was isolated from a municipal sewage sludge collected from Adrian, MI, USA [134]. Strain DCB-1 is able to reductively dehalogenate meta-halobenzoates [134, 135], and sulfate, sulfite and thiosulfate can also be use as electron acceptors, however, the presence of sulfuroxy anions inhibited dechlorination as well as high concentrations of formate and $\mathrm{H}_{2}$ [135]. Fermentation was observed with pyruvate supplied as sole carbon source [11]. Thiamine, nicotinamide and 1,4naphthoquinone are required in the defined media, and $\mathrm{MoO}_{4}{ }^{2-}, \mathrm{SeO}_{4}{ }^{2-}$, tetracycline, chloramphenicol, kanamycin and streptomycin are potential inhibitors [11]. Additionally, cytochrome $c_{3}$ and desulfoviridin were detected in the growth medium of Desulfomonile tiedjei [11].

Desulfomonile limimaris is another organohalide-respiring species with two strains DCB-M ${ }^{\mathrm{T}}$ and DCB-F that were isolated from marine sediments [56]. These two strains share 99\% similarity based on 16S rRNA gene sequences and are both capable to dechlorinate 3- 
chlorobenzoate $(\mathrm{CB})$ to benzoate with formate and $\mathrm{H}_{2}$ as electron donor. A broad range of electron donors can be used such as benzoate, $\mathrm{H}_{2}$, formate, butyrate, lactate, propionate and pyruvate. Besides 3-CB, sulfite, sulfate, thiosulfate, nitrate, and fumarate can be used as electron acceptors for growth. Compared to Desulfomonile tiedjei DCB-1 [11], these two isolates of Desulfomonile limimaris are not able to dechlorinate meta-halobenzoates using acetate or ferment pyruvate, and $\mathrm{NaCl}(>0.32 \%, w / w)$ is required for their growth [133]. Sulfite and thiosulfate and not sulfate inhibit OHR of Desulfomonile tiedjei DCB- $1^{\mathrm{T}}[11,136]$ and Desulfomonile limimaris $\mathrm{DCB}-\mathrm{M}^{\mathrm{T}}$, which was explained by the competition for electron flow.

\section{Desulfovibrio}

Desulfovibrio is a genus of Gram-negative sulfate-reducing bacteria (SRB) that belongs to the Proteobacteria (Phylum), Deltaproteobacteria (Class), Desulfovibrionales (Order) and Desulfovibrionaceae (Family). Up to now, two isolates of Desulfovibrio spp. were published with OHR capability, and both were isolated from marine sediments with halophenols as electron acceptors. Desulfovibrio sp. TBP-1 was isolated from estuarine sediments of the Arthur Kill in the New York/New Jersey harbor with 2,4,6-TBP as electron acceptor and supplied with hydrogen and acetate [137]. Only ortho-bromophenols and/or bromophenols without adjacent bromines at para- position were dehalogenated with lactate, pyruvate, formate or hydrogen as electron donors. Strain TBP-1 accumulated acetate when lactate and sulfate were provided, which indicated that this microorganism is an incomplete oxidizer and $\mathrm{NaCl}$ is required for its growth [137]. The other isolate is strain $\mathrm{SF}^{\mathrm{T}}$, the type strain of species Desulfovibrio dechloracetivorans, which is able to couple acetate oxidation to OHR for growth [138]. Strain SF3 was isolated from San Francisco Bay sediment with acetate as electron donor and 2-CP as electron acceptor [138]. Several electron donors can be used to couple to OHR for growth, such as acetate, pyruvate, fumarate, propionate, lactate, ethanol and alanine but not with sulfate, and only ortho-chlorophenols like 2-CP and 2,6-DCP were dechlorinated [138].

Besides, Desulfovibrio desulfuricans, a sulfate-reducing bacterium, pre-coated with $\mathrm{Pd}^{0}$ was used as a biomass-supported chemical (bioinorganic) catalyst for dehalogenation of 2-CP and polychlorinated biphenyls with formate or $\mathrm{H}_{2}$ as an electron donor [139]. Enhanced dechlorination was observed using the bioinorganic catalyst while negligible chloride release occurred from organohalides using biomass alone, chemically reduced or commercially available $\mathrm{Pd}^{0}$ [139]. The same concept was used to dechlorinate tris(chloroisopropyl)- 
phosphate (TCPP, a potential flame retardant) in a groundwater sample [94].

\section{Epsilonproteobacteria}

\section{Sulfurospirillum}

Sulfurospirillum, a genus of Gram-negative, elemental sulfur-reducing spirilla, belongs to the Proteobacteria (Phylum), Epsilonproteobacteria (Class), Campylobacterales (Order) and Campylobacteraceae (Family) [140]. The first OHRB characterized in this genus was Dehalospirillum multivorans strain K, a strictly anaerobic PCE-dechlorinating bacterium isolated from activated sludge of a wastewater treatment plant with pyruvate and PCE [141], which was later renamed as Sulfurospirillum multivorans [142]. Additionally, strain PCE-M2 ${ }^{\mathrm{T}}$, an anaerobic bacterium that is also able to reductively dechlorinate PCE to cis-DCE via TCE with lactate as electron donor, was isolated from a site polluted with chlorinated aliphatics in the Netherlands, and was assigned as a novel species Sulfurospirillum halorespirans to the genus Sulfurospirillum [142]. $\mathrm{AsO}_{4}{ }^{3-}, \mathrm{SeO}_{4}{ }^{2-}, \mathrm{PCE}$, nitrate and fumarate can serve as electron acceptors for strains K and PCE-M2 ${ }^{\mathrm{T}}[142,143]$. Strain PCE-M2 ${ }^{\mathrm{T}}$ is able to reduce nitrite while strain $\mathrm{K}$ is not, and the end product of nitrate reduction for strain $\mathrm{PCE}-\mathrm{M} 2^{\mathrm{T}}$ and strain $\mathrm{K}$ is ammonium and nitrite, respectively [142]. Pyruvate, lactate, $\mathrm{H}_{2}$ and formate can be used as electron donors to support PCE dechlorination, and fermentative growth was detected on pyruvate and fumarate [142]. Unlike all described RDases [46], PCE RDase of S. multivorans needs a special corrinoid cofactor (norpseudo-B12) [144] synthesized de novo by the strain itself [145].

\section{Gammaproteobacteria}

\section{Shewanella}

Shewanella belongs to the Proteobacteria (Phylum), Gammaproteobacteria (Class), Alteromonadales (Order) and Shewanellaceae (Family). Shewanella sediminis strain HAW$\mathrm{EB}^{\mathrm{T}}$ is a psychrophilic rod-shaped marine bacterium isolated from Halifax Harbour sediment, Canada $[146,147]$. This strain was originally noted for its ability to degrade hexahydro-1,3,5trinitro-1,3,5-triazine $(\mathrm{RDX})$ [146, 147], and its OHR capacity was later shown for PCE dechlorination to TCE [148]. This strain grows on several carbon sources such as N-acetyl-Dglucosamine, Tween 40, Tween 80, acetate, succinate, butyrate and serine [147]. Bacteria belonging to the Shewanella genus are also known for their versatile electron-accepting capabilities using a complex electron transfer network composed mainly of c-type cytochromes as well as iron-sulfur proteins [149]. To cope with low temperatures of marine 
environments, psychrophilic strains of Shewanella seem to have adapted through a decreased genome $\mathrm{G}+\mathrm{C}$ content and an increased proportion of alanine, proline and arginine in proteins to increase protein structural flexibility [150]. Genomic analysis of strain HAW-EB3 ${ }^{\mathrm{T}}$ revealed the presence of genes encoding five putative RDHs similar to characterized RDases, of which Ssed_3769 was responsible for PCE transformation [148]. The OHR potential of this bacterium was proposed to play a significant role in halogen and carbon cycles as it can uniquely access organic carbon resources locked as organohalogens in sediments that may not be available to non-dehalogenating heterotrophic microbes [148].

\section{Betaproteobacteria}

\section{Comamonas}

Comamonas was defined as a genus belonging to the Proteobacteria (Phylum), Betaproteobacteria (Class), Burkholderiales (Order) and Comamonadaceae (Family). Comamonas sp. 7D-2 is the only isolate capable of OHR. It is a strictly aerobic bacterium that can completely degrade bromoxynil (a widely used brominated aromatic herbicide). This bacterium was recently isolated from a bromoxynil octanoate-contaminated soil [151]. The RDase (BhbA) of stain 7D-2, which catalysed the reductive debromination of 3,5-dibromo-4hydroxybenzoate (DBHB) to 4-hydroxybenzoate (HB), was characterized as the first RDase isolated from strict aerobes, with exclusive substrate specificity for haloaromatic compounds with halogen substitutions at the ortho position of a hydroxyl group [151]. Similar to RDase genes from known OHRB, $b h b A$ encodes two Fe-S cluster-binding motifs and is associated with a potential anchor protein encoding gene, however, unlike canonical RDases, it lacks a twin arginine signal peptide and B12-binding motif [151]. Widespread presence of putative RDHs homologous to BhbA in marine Proteobacteria and the fact that the $b h b A$ gene was located on a large plasmid (pBHB) and surrounded by transposase genes suggested horizontal acquisition of $b h b A$ from aerobic marine Proteobacteria [151]. Furthermore, the crystal structure of a similar aerobic RDase was recently published [152]. These elegant reports point to further catabolic plasticity of OHRB and indicates that the available OHRB represent the tip of the iceberg while a huge diversity awaits discovery. 


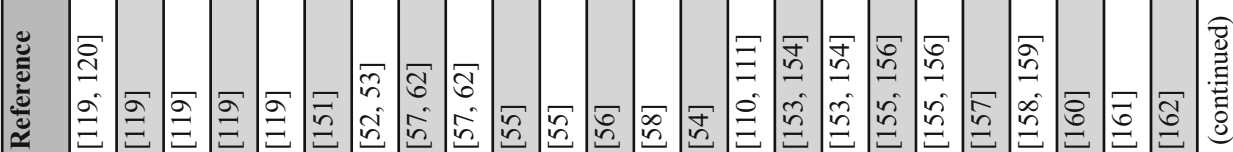

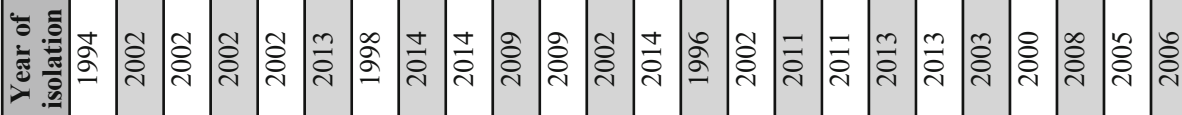

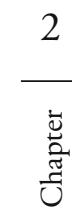

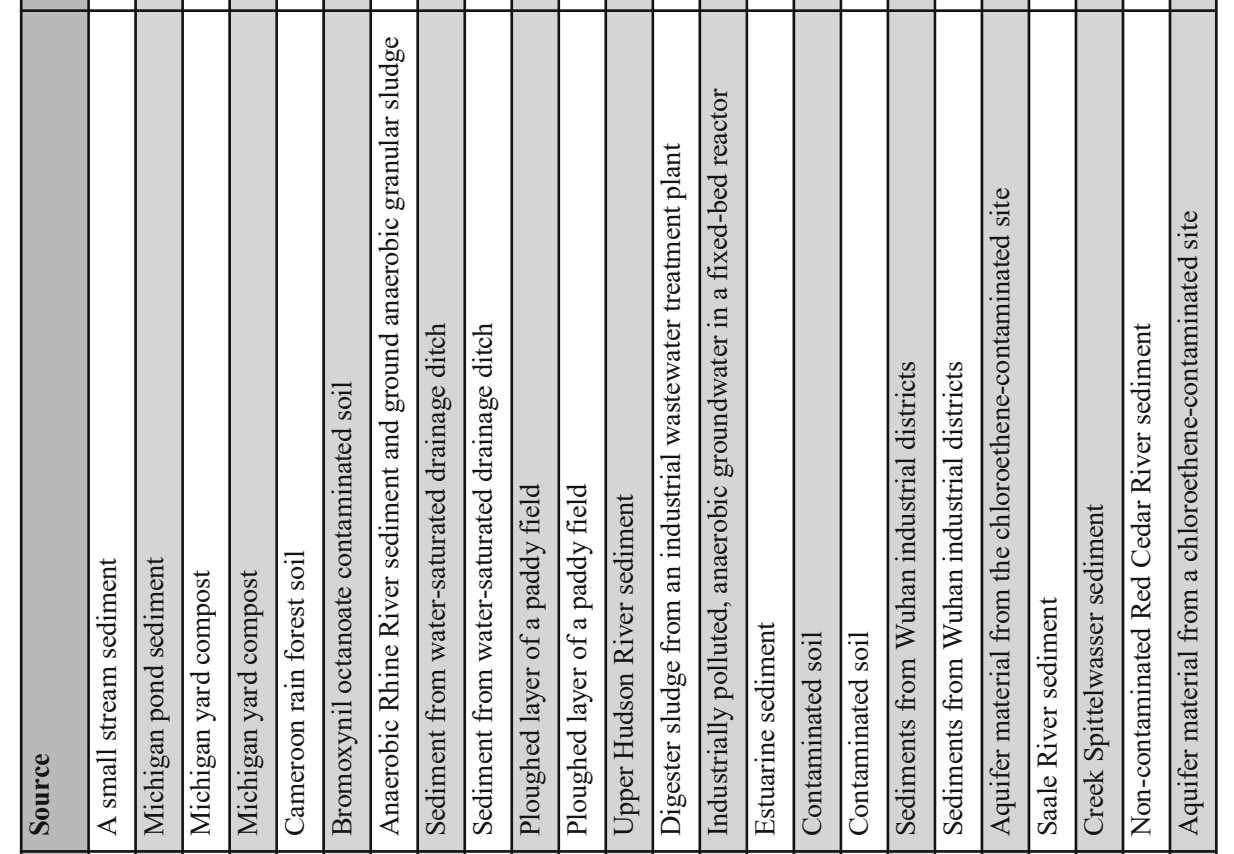

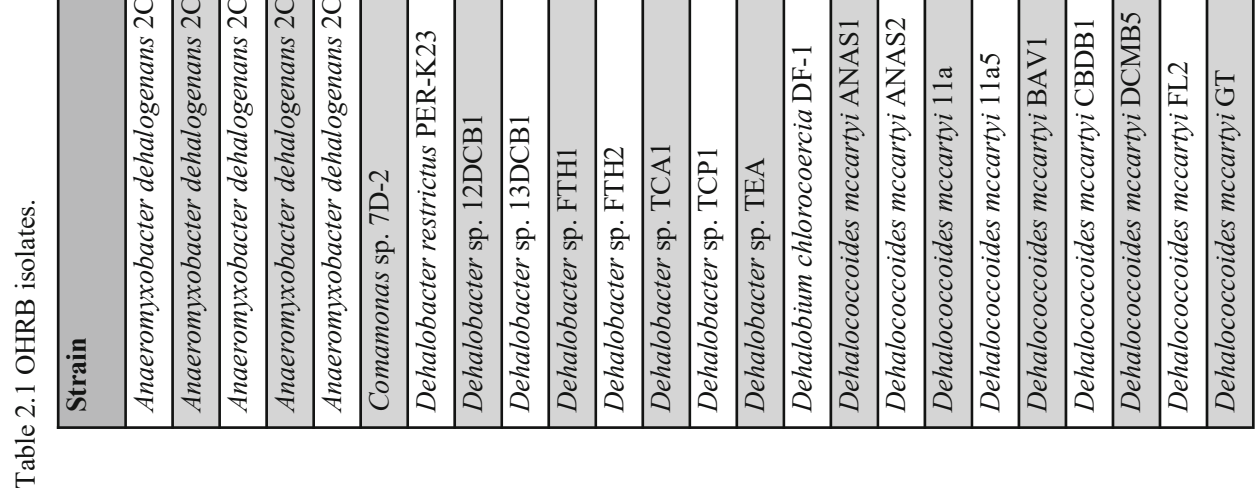

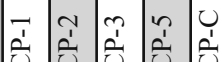

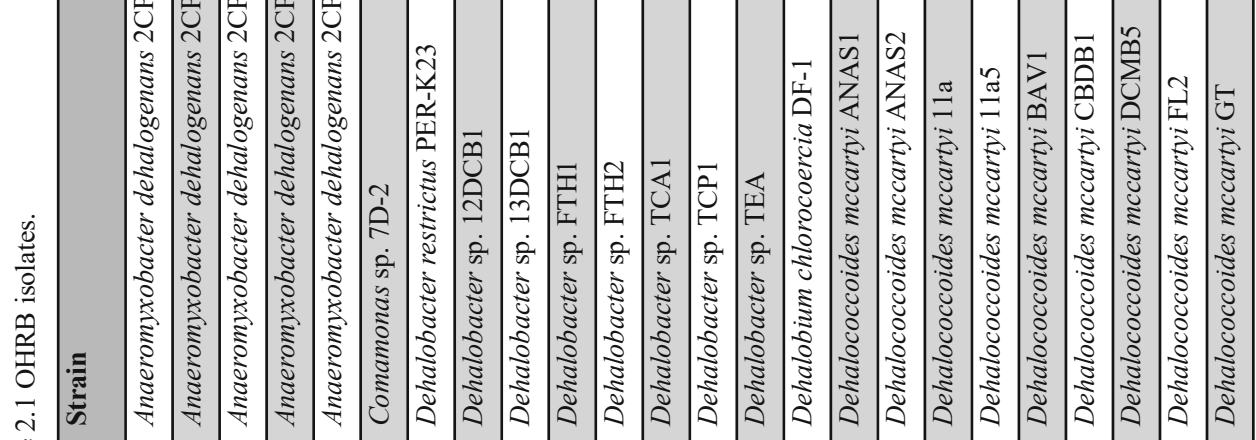




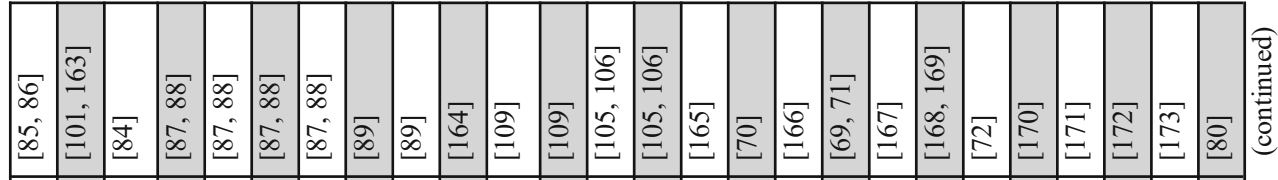

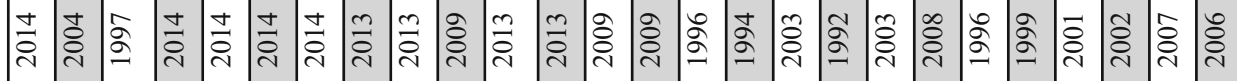

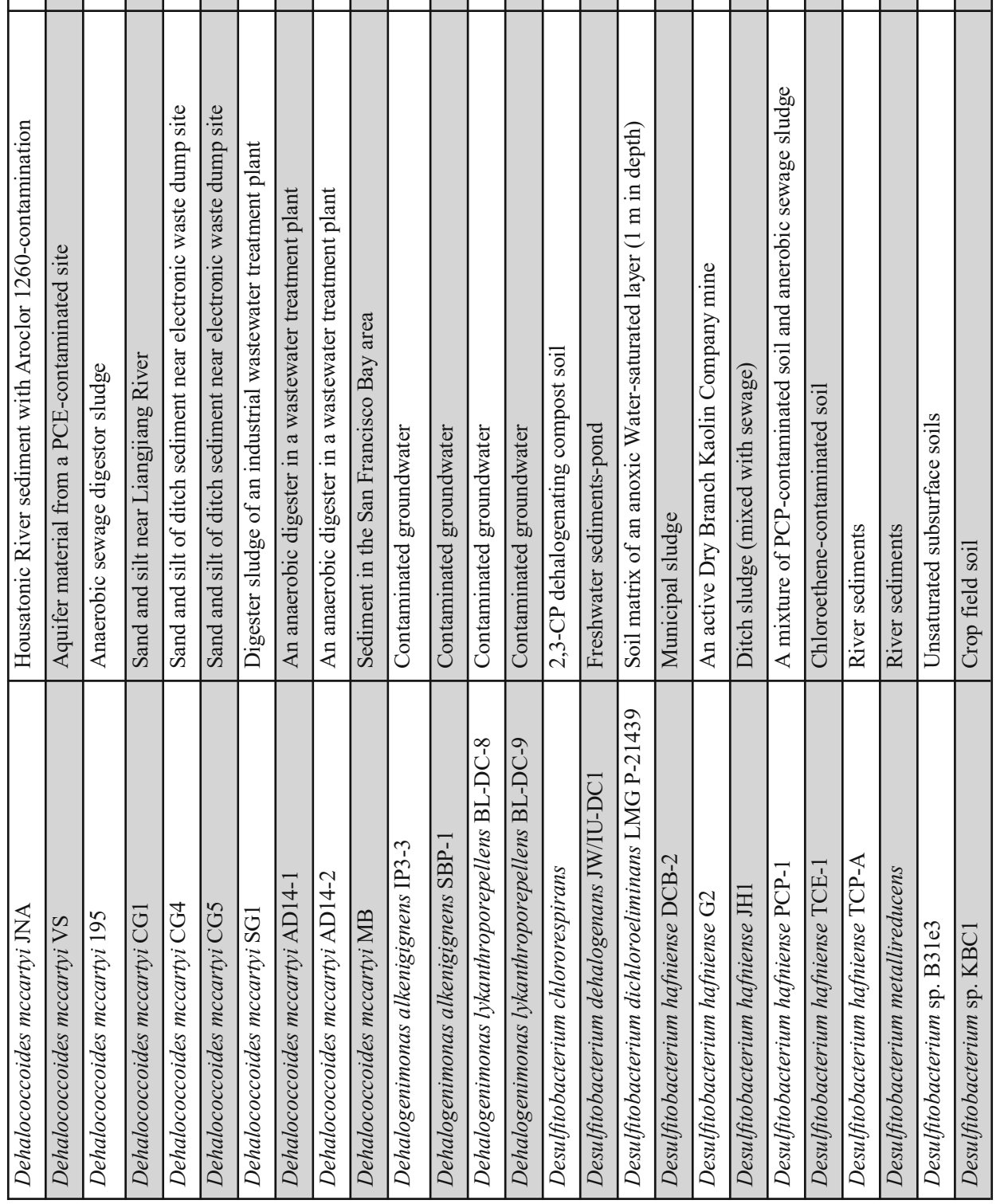




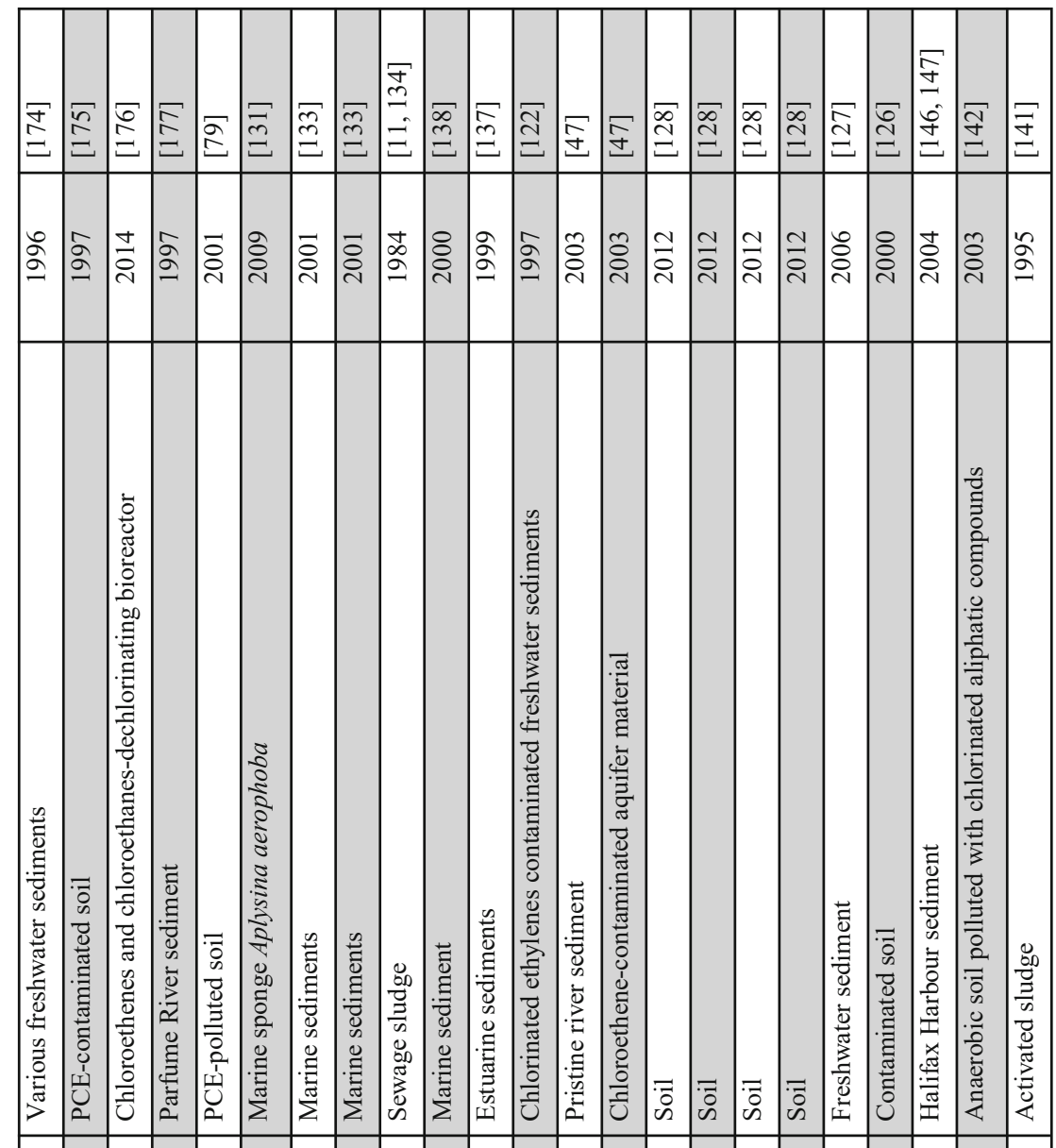

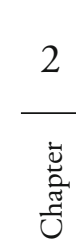




\section{Environmental distribution of OHRB}

Our current understanding of the environmental distribution of OHRB has to some extent been obtained by domestication of microbial communities capable of OHR in the form of laboratory culturing. Furthermore, monitoring of the presence of OHRB at a particular site was mediated by application of a suit of molecular techniques targeting phylogenetic and/or functional gene markers such as 16S rRNA and $r d h A$ genes. Combined with field data and compound specific isotope analysis (CSIA) as a tool for in situ monitoring of OHR, the allied application of these tools has created a complementary toolbox for examining the dehalogenation potential mediated by OHRB. Based on the worldwide detection of OHR potential, a cosmopolitan distribution of OHRB is likely, however, an overview of the geographical distribution of isolated OHRB indicates that nearly all OHRB were isolated from a restricted number of discrete parts of the world (Figure 2.3) though from different source environments (Figure 2.4). Most probably, this is not due to the restricted geographical distribution of OHRB, but rather due to the initiatives taken by a limited number of laboratories to isolate OHRB from these locations. Hence, a great diversity of OHRB awaits discovery that have not been studied from remote/ill-defined geographical locations. This fact is further illuminated in repeated isolation of different OHRB [42, 47, 161] and identification of OHR potential $[48,50,178,179]$ from pristine environments where naturally-occurring organohalides are believed to feed OHRB.

\section{Groundwater and aquifer}

The infamous popularity of OHR studies centred around groundwater and aquifer is due to anthropogenic contamination of these environments with organohalides as a serious threat to aquatic as well as terrestrial ecosystems [180-185].

In an early attempt to detect OHRB from aquifer samples, of the six different chloroethenecontaminated aquifer samples, only one tested positive for the presence of Dehalococcoides, and another tested positive for Desulfuromonas [186]. A similar study on a TCEcontaminated aquifer at Cape Canaveral Air Station, Florida, indicated heterogeneous distribution of dechlorinating activity and a specific dechlorinating organism, Dehalococcoides, at the site where the aquifer in question showed a non-uniform pattern of daughter product concentrations [187]. In a more comprehensive study, distribution of Dehalococcoides species was investigated in 10 groundwater samples collected from very diverse chloroethene-contaminated sites representing different geographical locations and a variety of climatic zones in North America, England, and The Netherlands [90]. 


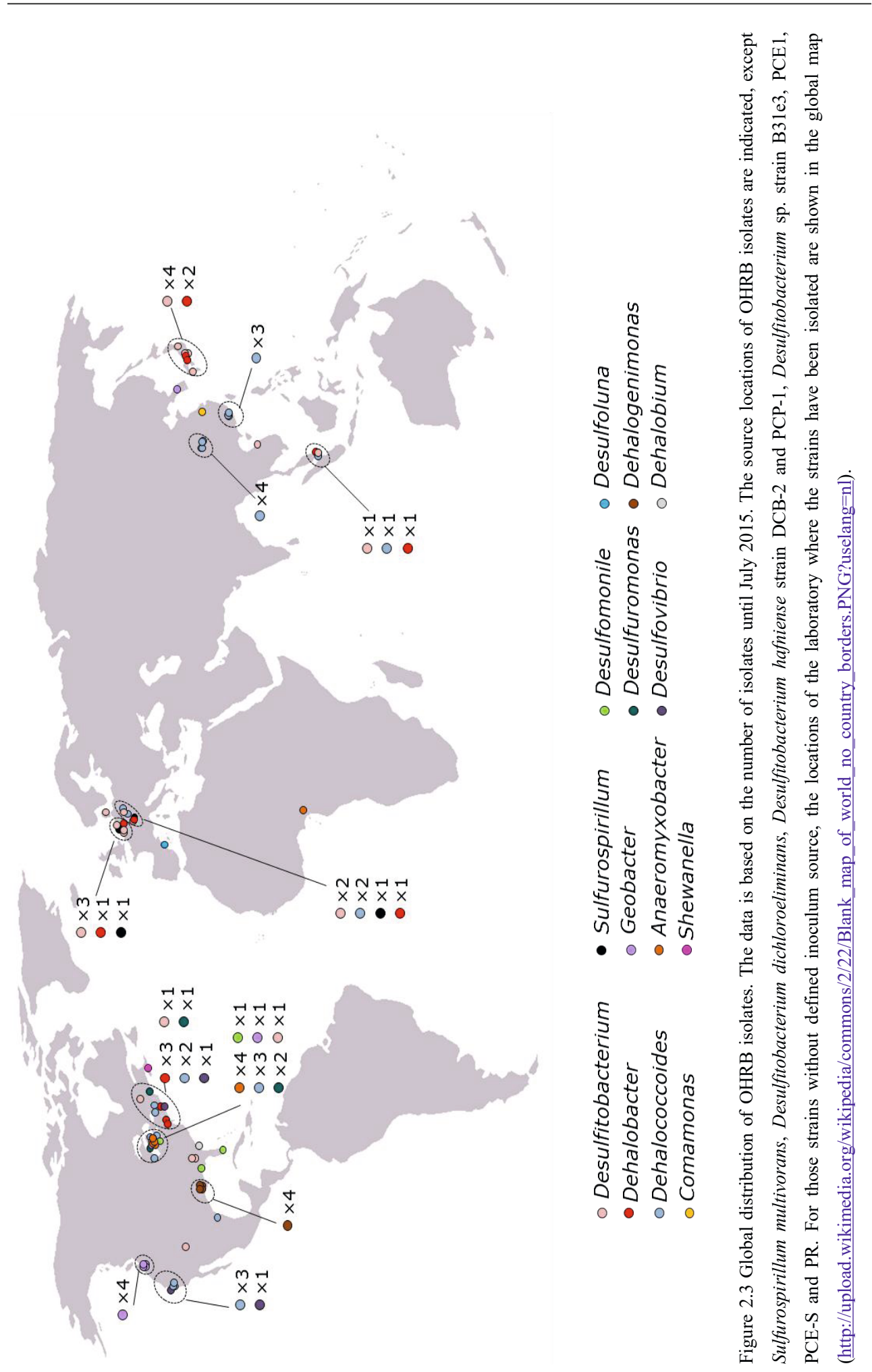




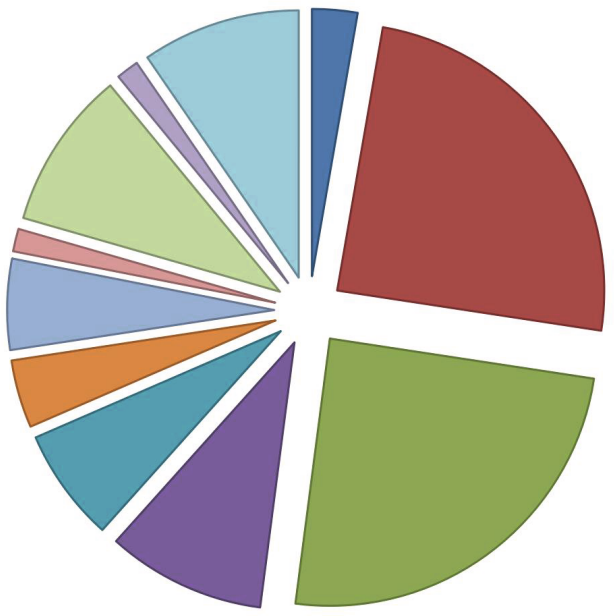

$\square$ mixture of sludge and soil

$\square$ soil

$\square$ terrestrial and freshwater sediment

$\square$ ditch and sludge

$\square$ aquifer material

$\square$ compost

$\square$ groundwater

$\square$ mine

$\square$ marine and estuarine sediment

$\square$ sponge

$\square$ reactor

Figure 2.4 Sources from which OHRB were isolated.

Dehalococcoides sequences were not detected in samples from three sites at which partial dechlorination of chloroethenes occurred [90]. While 16S rRNA gene-based PCR characterization failed to detect Dehalococcoides populations in aquifer samples from the Kelly AFB site (Texas, USA) before bioaugmentation with the KB-1 culture [188], 16S rRNA genes-based qPCR of Dehalococcoides and Desulfuromonas spp. were successfully used to monitor the distribution and abundance of the OHRB from groundwater and aquifer solids in the Bachman Road Residential Wells Site, USA [189]. In a study to track natural attenuation of a chlorinated ethene contaminated aquifer in the Bitterfeld/Wolfen area in Germany, various potential OHRB including Dehalococcoides, Desulfuromonas, Desulfitobacterium and Dehalobacter were detected providing an extra line of evidence for occurrence of natural attenuation [190]. In a wider survey of 34 environmental samples from 18 different sites (including 19 aquifer samples and eight groundwater samples) in different European countries, D. mccartyi and Desulfitobacterium spp. were detected concomitantly in the majority of the samples suggesting either in parallel or stepwise catalysis of complete dehalogenation of PCE by OHR [191].

The environmental distribution of OHRB in samples retrieved from aquifers contaminated with chlorinated ethenes was also shown by tracking the abundance and distribution of RDase genes with assigned functions, i.e. TCE-to-VC RDase gene (tceA) [192] and VC-to-ethene RDase gene ( $v c r A)$ [101]. The recovered $r d h A$ sequences from geographically distinct locations in the US exhibited between $95 \%$ and $98 \%$ identity with known $t c e A$ and $v c r A$ genes, respectively $[101,192,193]$. A similar approach was recently used for the RDase gene $d c p A$ 
mediating 1,2-dichloropropane dichloroelimination to propene [104]. The $d c p A$ gene was detected in all aquifer samples that yielded 1,2-D-dechlorinating microcosms [104], and $d c p A$ sequences retrieved from geographically distinct samples shared $>98 \%$ sequence identity with $d c p A$ sequences from D. mccartyi strains $\mathrm{RC}$ and $\mathrm{KS}[50,51]$ and D. lykanthroporepellens BL-DC-9 [105].

Studying the abundance and environmental distribution of OHRB was propelled by application of DNA/RNA-based quantitative PCR methods [194-196]. Using this method, the $16 \mathrm{~S}$ rRNA and $t c e A, b v c A$ and $v c r A$ genes of $D$. mccartyi were detected in groundwater samples collected from a contaminated site near Montague, MI, USA and a large number of unidentified D. mccartyi $r d h A$ genes were discovered [195]. Analysis of 45 groundwater samples taken from Rugårdsvej 234, Odense, Denmark for D. mccartyi 16S rRNA and vcrA genes indicated the presence of indigenous biomarkers at below detection limit of qPCR [197]. Increases in ethene concentrations after lactate addition were accompanied by a 3-4 order of magnitude increase in concentrations of vcrA gene copies throughout the groundwater monitoring network [197]. The oligotrophic nature of aquifers as one of the main obstacles of subsurface OHR was noted in similar studies [198, 199]. Quantification of transcripts of the same biomarkers in groundwater samples from the Fort Lewis military base in Tacoma, WA, USA also verified an indigenous community of $D$. mccartyi cells carrying the $t c e A, v c r A$, and $b v c A$ genes [194]. A less active physiological state of the $D$. mccartyi cells carrying tce $A$ was noted as opposed to the cells that harbour $v c r A$ and $b v c A$ [194] which was similar to a DNA based survey of an alluvial plain with complex geochemical and hydraulic conditions in France [200]. In an attempt to relate molecular data to geochemical conditions, 150 monitoring wells spread over 11 chlorinated ethene polluted European locations were analysed [201]. The vcrA gene copy numbers correlated most significantly to $\mathrm{VC}$ and chlorinated ethene concentrations while $b v c A$ and especially tce $A$ were more correlated with oxidizing conditions. The results showed geochemical conditions as the driver of geographical distribution of the RDase genes [201]. More recently, Dehalobacter was found to be more abundant than $D$. mccartyi populations in the clay till matrix collected from chlorinated ethenes and ethanes contaminated site in Vadsby, Denmark which corresponded with OHR being stalled at cis-DCE [202].

Cultivation independent approaches extended to subsurface aquifers further enhanced our understanding of metabolic plasticity of Dehalococcoidia-like bacteria. A recent metagenomic analysis from terrestrial aquifer sediment retrieved two Dehalococcoidia related 
pan-genomes ('RBG-2' and 'RBG-1351') [203]. Rather than OHR mediating $r d h A$ genes, versatile heterotrophic life styles were found [203]. These findings indicate that OHR should not be considered as sole mode of metabolism of Dehalococcoidia-like bacteria.

Various OHRB capable of respiration on diverse organohalides were isolated from groundwater and aquifer material as listed in Table 2.1.

\section{Marine and estuarine environments}

Marine and estuarine environments are repositories of numerous chlorinated, brominated and iodinated organic compounds that have been produced and released both from natural and anthropogenic sources. The study of King was the first report on OHR of 2,4-dibromophenol in coastal sediments [204]. Since then, numerous studies reported OHR as an essential step in the marine and estuarine biodegradation of halophenols [132], halogenated ethenes [148, 164], halobenzoates [133], lindane [205] and polychlorinated biphenyls [41, 206, 207].

Of all known OHRB, the organohalide-respiring Chloroflexi seem to play the most prominent role in OHR in marine, estuarine and tidal flat sediments. The ortho-PCB-dechlorinating bacterium $o-17$ was isolated from a $\mathrm{PCB}$ dechlorinating culture enriched from estuarine sediments of Baltimore Harbour (USA) [112]. Similarly, The PCB-dechlorinating Dehalobium chlorocoercia DF-1 is indigenous to estuarine sediment and was isolated from Charleston harbour in South Carolina [111]. An active role of these isolates and other Chloroflexi members that are distinct from D. mccartyi were shown in PCB dechlorination in sediment microcosms from Baltimore Harbour, USA [208]. Similar Chloroflexi phylotypes were reported for PCB dechlorination in enrichment from contaminated marine sediment of the Venice lagoon, Italy [209]. The Tidal Flat Cluster, a deep clade in the phylum Chloroflexi, was characterized form North Sea tidal flat sediment of the German Wadden Sea and was found to be involved in the dechlorination of PCE to trans-DCE as the predominant end product [49]. Molecular analysis of the bacterial community in enrichment cultures obtained from estuarine sediments of the San Diego Bay, USA, indicated involvement of Chloroflexilike microorganisms related to Dehalococcoides in OHR of the model dioxin, 1,2,3,4tetrachlorodibenzo-p-dioxin (TeCDD) [210].

In addition to former studies where detection of organohalide-respiring Chloroflexi was coupled to physiological/biochemical demonstration of OHR potential, there are other reports on abundant presence of bacteria affiliated with the Dehalococcoidia in marine sediments [211-213]. Wasmund and colleagues performed a comprehensive study on distribution and 
diversity of Dehalococcoidia-affiliated bacteria along vertical profiles of various marine sediment cores obtained from Baffin Bay (Greenland), Aarhus Bay (Denmark), and tidal flat sediments of the Wadden Sea (Germany) [214]. qPCR analysis showed that Dehalococcoidiaaffiliated bacteria constituted only low proportions of the total bacterial in shallow sediment and generally became more prevalent in deeper sediments. Pyrosequencing of 16S rRNA genes of different clades revealed diverse and divergent co-occurring Dehalococcoidia populations within single biogeochemical zones. The varying local biogeochemistry was proposed as the driving force for such abrupt shifts. Hence different subgroups of Dehalococcoidia-affiliated bacteria with different metabolic properties likely occupy a wide range of ecological niches over sediment depth [214].

A number of reports exist on distribution and diversity of $r d h A$ genes in marine subsurface sediments. Using an array of degenerate primer sets, 32 putative $r d h A$ phylotypes were detected from marine subsurface sediments collected from the southeast Pacific off the Peruvian coast, the eastern equatorial Pacific, the Juan de Fuca Ridge flank off the coast of Oregon, and the northwest Pacific off Japan down to a depth of $358 \mathrm{~m}$ below the seafloor [178]. OHR was noted using 2,4,6-tribromophenol and trichloroethene in sediment slurry collected from the Nankai Trough [178]. During another study on sediments of the northwest Pacific off the Kii Peninsula of Japan, OHR was restricted to 4.7 metres below the seafloor, despite detection of $r d h A$ genes in deeper sediments [215]. A recent metagenomic survey on deep sub-seafloor further broadened the diversity of currently known $r d h A$ genes [216]. These results are in contrast to single cell genomic studies of marine Dehalococcoidia where no evidence for OHR were found [44, 45]. These studies indicated that microorganisms closely related to known obligate OHRB do not rely on OHR for energy conservation, but rather utilize organic matter degradation pathways which is in line with a similar study from aquifer materials [203]

Marine and estuarine environment has been an important source for the isolation of OHRB given their likely exposure to abundant organohalides and remarkable distribution of $r d h A$ genes. Various OHRB isolates with diverse OHR capabilities were obtained from marine sediment as outlined in section 5.2, and a comprehensive compilation is given in Table 2.1.

\section{Riverbed and lake sediments}

Due to the high diversity of organisms, the high load of organic nutrients and strong reducing power, sediment geochemical conditions strongly differ from those observed in aquifers, and 
thus can support a wide diversity of OHRB. The first study on OHR potential from a lake sediment and sewage sludge was conducted by one of the pioneering labs of the OHR field, Tiedje and co-workers [217]. The observed reductive dehalogenation in the obtained enrichment culture was the first report of OHR as a novel anaerobic respiration pathway that has profoundly altered our perception of biodegradability of organohalides especially under anoxic conditions [217]. The methanogenic consortium was capable of dehalogenating and often mineralizing a variety of halobenzoates to $\mathrm{CH}_{4}$ and $\mathrm{CO}_{2}[217,218]$. Numerous studies reported anaerobic dechlorination of chlorinated ethenes in the groundwater surface water interaction zone from various geographical locations i.e. freshwater tidal wetlands of West Branch Canal Creek, Maryland, USA [219], Lake Michigan, USA [220], as well as streambed sediments in Angus, Ontario, Canada [221] and in Vilvoorde, Brussels, Belgium [222] without identification of responsible microorganisms. With growing interest on environmental distribution of OHRB, several 16S RNA gene-based methods were applied to track their presence in organohalide impacted or pristine sediments [48, 90, 186, 223]. In early attempts to detect OHRB from sediment environments, sediment samples of three pristine Michigan rivers yielded amplification products with Desulfuromonas- and Dehalococcoides-targeted primer sets independent of the sampling season [186]. RNA-based stable isotope probing was applied for detection of novel PCE to cis-DCE respiring OHRB from pristine river sediment of the river Lahn, Marburg, Germany [48]. These novel OHRB within the phylum Chloroflexi were only distantly related to cultivated $D$. mccartyi strains (92-94\% sequence identity) [48]. In a chlorinated ethene impacted streambed in Toronto, Canada, D. mccartyi guilds were detected where soil organic contents were above $2 \% \mathrm{w} / \mathrm{w}$ and where transformation proceeded to ethene [223].

OHR in the riverbed sediments of the Zenne river, Brussels, Belgium impacted with cis-DCE, VC and 1,1-dichloroethane (1,1-DCA) coincided with the presence of Dehalobacter and D. mccartyi guilds [224]. Similar presence of Dehalobacter [225] and D. mccartyi [225, 226] was noticed in a number of follow up studies using microcosms prepared with material from the same site, whereas no Desulfitobacterium was detected. In both microcosm studies, VC RDase encoding $v c r A$, and $b v c A$ genes were the most abundant $r d h \mathrm{~A}$ genes [225, 226]. However, in spite of repeated detection of biomarkers associated with OHRB in the field, microcosm studies with Zenne riverbed sediments [224-226] and high OHR performance in microcosm studies with material from different locations at the Zenne river site [222, 226], high spatial variability of OHR potential was observed in field measurements from the 
riverbed [224]. Hence, rather than the microbial community structure of the Zenne River sediments, the $\mathrm{CAH}$ discharge rate was hypothesized as the limiting factor for OHR in the Zenne sediments [227]. The high discharge rate and hence reduced residence time of discharging contaminated groundwater seems to be an important prerequisite to drive $\mathrm{OHR}$ within riverbed sediments [223, 227].

The composition and abundance of active OHRB was investigated in riverbed sediments collected from the Ebro river in Spain and the Elbe river in Germany [228]. D. mccartyi and Desulfitobacterium 16S rRNA were detected at the sampling locations whereas Dehalobacter rRNA could not be detected. D. mccartyi populations were more active and had a higher diversity in river sediments than in floodplain soils in the Elbe River and in general were more dominant in HCB-polluted locations within river basins than Desulfitobacterium spp. and Dehalobacter spp., albeit with significant temporal and spatial variation [228]. In a recent study 18 sediment samples from three different locations in the Yangtze River, China, were tested for OHR potential [229]. PCE to cis- and trans-DCE dechlorination was concomitant with increasing numbers of Dehalobacter spp., Desulfomonile spp., Desulfitobacterium spp., or D. mccartyi [229]. Krzmarzick and co-workers detected putative organohalide-respiring Chloroflexi in 67 of 68 samples collected from lake sediments across a geographical sulfur gradient. Higher quantities of these bacteria were noted where lower sulfate concentration was recorded, indicating an antagonistic relationship between both functional guilds [229].

\section{Soil}

Ubiquitous distribution and active involvement in halogen cycles was also shown for organohalide-respiring Chloroflexi in soil. An interesting study by Krzmarzick [179] documented widespread distribution of organohalide-respiring Chloroflexi in 116 pristine soil samples collected from different locations in USA where their number of 16S rRNA genes positively correlated with organochlorine]/TOC [179]. Triclosan addition to agricultural soil from Fairfax, Minnesota, USA, without previous exposure to this substrate increased abundance of Dehalococcoides-like Chloroflexi in soil microcosms [230]. Furthermore, recently the enrichment of a TCE respiring consortium from garden soil collected from Cuzdrioara, Cluj County, Romania, was reported [231]. In spite of the presence of $D$. mccartyi and tce A, vcrA and $b v c A$ genes, dechlorination stalled at cis-DCE even after repeated biostimulation with lactate. The authors hypothesized that this might be a consequence of the intrinsic competition for electron donor $\left(\mathrm{H}_{2}\right)$ in soils and sediments, driven by a variety of electron acceptors such as nitrate, Fe (III), sulfate, and bicarbonate [231]. 
Besides the abundant OHRB belonging to Chloroflexi, important roles played by the OHRB belonging to Firmicutes were reported in terrestrial soil environments. Geographic distribution of Desulfitobacterium hafniense PCP-1 and other Desulfitobacterium spp. was studied in 44 soil samples from Quebec, Canada, using a PCR method showing ubiquitous presence of Desulfitobacterium spp. [232, 233]. A survey of 65 soil samples from chloroethene-contaminated and pristine soils in Japan showed PCE to cis-DCE dechlorination in 16 out of 44 chloroethene-contaminated soils and three out of 21 pristine soils [173]. PCE dechlorination was mostly associated with detection of Desulfitobacterium spp. and probably Dehalobacter spp. rather than D. mccartyi [173]. Complete pentachlorophenol (PCP) to phenol degradation was noted in an enrichment culture obtained from paddy field soil located at Yatomi-cho, Aichi prefecture, Japan [234]. The involvement of Gram-positive sporeforming bacteria belonging to the phylum Firmicutes was proposed, although Desulfitobacterium spp. were not detected by PCR using a specific primer set [234]. The same soil samples were later shown to be conducive to dechlorination of fthalide where Dehalobacter sp. strains FTH1 and FTH2 were identified as potential OHRB [55]. Involvement of novel Firmicutes referred to as Gopher group was also proposed in the dechlorination of two chlorinated xanthones, analogues of natural organochlorines in pristine forest soil from New Jersey and pristine sediment samples collected from lake sediment in Minnesota, USA [235]. The Gopher group was shown to be distinct from but related to Dehalobacter and Desulfitobacterium [235].

\section{Factors impacting environmental distribution of OHRB}

Of particular importance for the distribution and fate of OHRB (and hence organohalides) in the environment are redox conditions, availability of micronutrients, the presence of suitable electron donors and acceptors and the interaction with chemical species.

\section{Redox conditions}

With the exception of Comamonas sp. 7D-2 as a strict aerobic bacterium capable of OHR [151] and Anaeromyxobacter dehalogenans as a facultative anaerobe [119], the currently known OHRB are generally strictly anaerobic bacteria and sensitive to oxygen exposure. This is of high importance for the more delicate OHRB such as Dehalococcoides and Dehalobacter spp. that require strict anoxic techniques and presence of reducing chemicals in laboratory cultures. Lacking the genes necessary for direct utilization of oxygen, these OHRB are highly dependent on an array of natural shields in the environment. Recent findings showed that in spite of stringent requirement for anoxic conditions in axenic cultures, 
members of Dehalococcoides might be well protected in their natural habitats such as riverbed sediments [226]. The organic rich matrix of riverbed sediments limits oxygen penetration to the surficial sediment layers. Moreover, sediment aggregates can host anoxic microniches inside sediment-associated biofilms. Presence of facultative aerobic bacteria colonizing the outer layers of sediment biofilms, which rapidly consume oxygen, can protect the strict anaerobes such as OHRB in core microniches [226]. In line with this, a former study on distribution and localization of D. mccartyi 195 and methanogenic populations in an enrichment culture using fluorescence in situ hybridization (FISH) showed that methanogens reside in the exterior layer of bioflocs, whereas D. mccartyi 195 cells were more evenly distributed within bioflocs as well as between biofloc and planktonic phases [236]. A comparative metagenomic study on three different Dehalococcoides-containing dechlorinating microbial consortia indicated the presence of several genes related to oxygen tolerance and scavenging from acetogens, fermenters and methanogens commonly found to co-exist with OHRB in enrichment cultures and natural environments [237]. Activity of such genes in non-dehalogenating community members would enforce oxygen scavenging mechanisms, collaterally protecting the OHRB along-side.

\section{Micronutrients}

OHRB also depend on other non-dehalogenating community members for the supply of electron donor(s) and growth factors such as vitamins. Fermentation intermediates such as acetate, formate, and $\mathrm{H}_{2}$ are common products of anaerobic hydrocarbon degradation [238], and are used by other community members such as iron reducers, SRB, OHRB and methanogens. This overlapping nutritional niche caused controversial discussions in the field of OHR. Early reports considered hydrogenotrophic SRB and methanogens as competitors of OHRB for reducing equivalents [239, 240], however, the (predicted) supply of nutrients by SRB and methanogens [237] points towards interactive networks between these microbial guilds rather than pure competition for the shared resources. Indeed, the importance of symbiotic interactions between SRB and OHRB for effective OHR in their co-cultures was previously reported and was attributed to interspecies $\mathrm{H}_{2}$ transfer [241, 242] or supply of unknown growth factors by the SRB partner [243]. Recent dedicated studies showed that supply of corrinoid cofactors by SRB and methanogens sustained OHR by D. mccartyi 195, although corrinoid modification and remodelling by strain 195 was essential to add 5',6'dimethylbenzimidazole (DMB) as the lower ligand to cobamide precursors and produce cobalamin as the preferred corrinoid cofactor [93-95]. Similarly, Geobacter lovleyi strain SZ 
was shown to fulfil Dehalococcoides' corrinoid requirements by the exchange of DMB [93]. Given the fact that all D. mccartyi strains for which genome sequences are available lack the capability of de novo cobalamin synthesis [96], and all share the same putative genes involved in corrinoid-remodelling pathways [94], it is tempting to assume that OHRB exploited the biotopes shared with non-dechlorinating community members and have lost the costly but still key metabolic trait of de novo cobalamin synthesis. In line with this, recent studies on corrinoid-auxotrophy of Dehalobacter restrictus showed that in spite of presence of both anaerobic cobalamin biosynthesis and cobinamide-salvaging pathways, one important protein, $\mathrm{CbiH}$ (precorrin-3B C17-methyltransferase), was missing from the upper cobalamin biosynthesis pathway, which was believed to be due to frame-shift mutations [65]. Supply of the OHRB with corrinoids in their natural environment or even during long-term enrichments in the lab may enforce such a genetic trade-off that disables the unused trait. This may explain why OHRB and in particular those who rely on OHR as the sole mode of metabolism have shared/overlapping niches with non-dechlorinators that are capable of de novo corrinoid biosynthesis. Experimental evidence also suggest that OHRB are mostly enriched in methanogenic and sulfate-reducing conditions. For example, a recent batch-scale biostimulation of TCE dechlorination showed incomplete TCE dechlorination in microcosms where methanogens were absent [199]. In another study, inhibition of methanogenesis negatively impacted community members such as Desulfovibrio [244] that are known to synthesize corrinoids de novo.

In natural habitats such as cell aggregates and biofilms where cells form close physical association, OHRB and methanogens may play complementary roles in alleviating thermodynamic constraints for other syntrophic microorganisms. The degradation of fatty acids such as propionate and butyrate only takes place in a thermodynamically interdependent lifestyle where degradation intermediates, usually acetate, formate, and $\mathrm{H}_{2}$, are maintained at very low concentrations [238]. Such metabolic/physical networks may also explain why most fastidious OHRB like D. mccartyi strains do not submit readily to growth in the laboratory as they need metabolites, signals and structure provided by the community. Further, the structured associations would be more successful in retaining metabolites such as $\mathrm{H}_{2}$ that would otherwise be lost by diffusion. However, in spite of the central role of microbe-microbe interactions, this subject has received surprisingly little attention in studies involved in OHR. Such information would highly assist in understanding the ecophysiology of OHRB and facilitate design of effective and sustainable bioremediation strategies. 


\section{Electron acceptors}

The presence of the right terminal electron acceptor is vital for the success of this process as not all OHRB are dedicated to OHR. It is not known whether facultative OHRB would prefer other electron acceptors over organohalides in their natural environment although some general assumptions can be made. At higher redox potential the facultative OHRB such as proteobacterial OHRB may prefer electron acceptors like nitrate, $\mathrm{Mn}(\mathrm{IV})$, and $\mathrm{Fe}(\mathrm{III})$. A recent study on correlations between environmental variables and bacterial community structures indicated that both $\mathrm{VC}$ and $\mathrm{Fe}(\mathrm{III})$ reduction were most important but antagonistic terminal electron-accepting processes [245]. Once highly reducing conditions prevail, organohalides may act as electron acceptors. However, the organohalides in natural habitats may not be as readily accessible as in well-homogenised lab cultures. For example, higher degree of halogen substitution can reduce bioavailability of organohalides. This is particularly important in soil and sediment matrices that contain strong sinks of organohalides such as amorphous organic carbon and black carbon [246]. In such environments, highly halogenated and hydrophobic organohalides such as dioxins and PCBs adsorb strongly to organic matter, reducing their bioavailability to OHRB [247]. It should however be noted that such a reduced bioavailability will also lower the acute toxicity of organohalides to OHRB, hence playing a double-edged sword function. Besides, not all types of organohalides are degraded by all OHRB. The facultative OHRB known to date do not dechlorinate PCE past DCE. Even within D. mccartyi strains, except for genomic evidence for the strain BTF08 [248], no other isolate is capable of complete PCE dechlorination [40]. However, multiple OHRB may reside in close proximity and co-exist in the same habitat, which is necessary for their robust OHR activity by complementary dehalogenation activity. For example, the concomitant presence of bacteria belonging to D. mccartyi and Desulfitobacterium groups was previously reported in chloroethene-dechlorinating microbial communities [191]. Another study with an enrichment culture showed that Sulfurospirillum-affiliated bacteria were involved in PCE dechlorination to cis-DCE whereas Dehalococcoides-affiliated bacteria mainly dechlorinated cis-DCE to ethene [249].

\section{Interaction with chemical species}

Interaction of OHRB with abiotic factors is highly important for their fate in environment. The electrophilic nature of organohalides and reductive nature of the OHR process demands efficient electron transfer mechanisms. Naturally occurring electron shuttles and redox mediators that can be reversibly oxidised and reduced have the capacity to serve as external 
electron carriers in redox processes such as OHR [250]. Humic substances and their quinoid analogues are among the best studied natural redox mediators in promoting OHR [251, 252]. Under oxygen-depleted conditions in the subsurface, these redox mediators can be reduced not only by combustion of organic substrates, but also by chemical reactions with reductants common to anaerobic soils and sediments such as Fe(II), sulphide, and cysteine. The electronwithdrawing organohalides can in turn be reduced in subsequent OHR thereby re-oxidising the mediators. Hence, a cocktail of biotic and abiotic reactions could indirectly stimulate OHRB. The organohalides were also shown to be abiotically degraded by naturally-occurring minerals such as pyrite, magnetite and green rusts [253]. Biogenic abiotic catalysts such as palladium nanoparticles can also effectively remove organohalides such as TCE [254]. The abiotic reductive dechlorination reaction could be complementary to OHR at places where the condition is less favourable for OHRB and hence compensate for their drawbacks.

\section{Conclusions}

The currently known OHRB belong to distinct phylogenetic groups of deeply branching Chloroflexi, Gram-negative Proteobacteria and Gram-positive Firmicutes. The ubiquitous documentation of OHR potential in aquatic as well as terrestrial environments indicates that the current collection of OHRB is only the tip of the iceberg. It is likely that a considerable portion of OHRB has to date been ignored during conventional culturing techniques. Recent advances in high-throughput culturing approaches should pave the way for isolation of novel OHRB, bypassing many obstacles of conventional cultivation. Moreover, most studies to date were performed using model chlorinated compounds of different haloaliphatic and haloaromatic classes. This limited spectrum of substrates in part is due to simplicity of handling these rather "classical" organohalides and the fact that most of complex naturally occurring organohalides are not commercially available. The known OHRB show moderate traits with regards to extremes of temperature (except for the psychrophilic marine bacterium, Shewanella sediminis [147]), $\mathrm{pH}$, and salinity and up to now, there has been no report on OHRB isolated from (poly)extreme environments. Moreover, the OHR capacity has been restricted to the bacterial domain of life and no archaeon has been reported to be associated with OHR in axenic cultures. This is despite the fact that they have been repeatedly found in close association with OHRB in enrichment cultures and environmental samples. 


\section{Acknowledgements}

Siavash Atashgahi, Yue Lu and Hauke Smidt thank the BE-Basic Foundation (project

MicroControl (8.004.01)) for financial support. Yue Lu was sponsored by a CSC Fellowship. 


\section{Primers that target functional genes}

\section{of organohalide- respiring bacteria}

This chapter has been published: Yue Lu\#, Siavash Atashgahi\#, Laura Hug, Hauke Smidt. 2015. Primers that target functional genes of organohalide-respiring bacteria. In: Hydrocarbon and Lipid Microbiology Protocols, 


\begin{abstract}
Halogenated organic hydrocarbons are problematic environmental pollutants that can be reductively dehalogenated by organohalide-respiring bacteria (OHRB) in anoxic environments. This energy-conserving process is mediated by reductive dehalogenases (RDase). To amplify the diversity of reductive dehalogenase-encoding genes, degenerate primers have been designed, most of which target the conserved regions of the encoded protein sequences of the catalytic subunit, RdhA. In addition, specific primer sets have been developed and widely used to quantify and characterize OHRB and the reductive dehalogenase homologous $(r d h)$ genes in the environment. The specific primers have been applied to multiple molecular techniques including regular and quantitative PCR, Southern blot hybridization, terminal restriction fragment length polymorphism (T-RFLP), and reverse transcriptase PCR (RT-PCR). The hunt for novel $r d h A$ genes has benefited greatly from next generation sequencing techniques, including primer-dependent amplicon sequencing and primer-independent metagenomic analyses. This chapter provides an overview of most primers targeting RDase-encoding genes described to date and their applications, and it discusses the developing trend of leveraging primer-(in)dependent techniques for better understanding of OHRB and their RDase gene pools.
\end{abstract}




\section{Introduction}

Halogenated organic compounds (organohalides) are problematic environmental chemicals that over the last century have been widely produced and used for industrial applications and chemical manufacturing. As a result of accidental or indiscriminate disposal, organohalides are among the most abundant soil and groundwater contaminants. Microorganisms have evolved different strategies to take advantage of organohalides, greatly impacting the compounds' environmental fates. Under anaerobic conditions, organohalides can be reductively dehalogenated, which, depending on the degree of dehalogenation, can lead to their detoxification or render them susceptible for further (bio)transformation [10]. During this process, known as organohalide respiration (OHR), the organohalides are used as terminal electron acceptors in an energy-conserving respiratory metabolism. The chemically stable halogen-carbon bond is unlocked by replacing the halogen atom with hydrogen and liberating it as a halide [46]. This process is mediated by organohalide-respiring bacteria (OHRB), some of which are extreme niche specialists with OHR as their only metabolism. This group of obligate OHRB comprises the organohalide-respiring members of Chloroflexi (including strains of Dehalococcoides mccartyi, Dehalogenimonas, and Dehalobium) and Firmicutes (strains of Dehalobacter) [13, 46]. It should however be noted that recent studies showed fermentative growth of a few Dehalobacter spp. on chloromethane [42, 43]. Moreover, single cell genomic studies of marine Dehalococcoidetes did not reveal any evidence for catabolic reductive dehalogenation, indicating that microorganisms closely related to known obligate OHRB do not rely on OHR for energy conservation, but rather utilize organic matter degradation pathways [44, 45]. Compared to obligate OHRB, facultative OHRB have versatile metabolisms encoded by relatively large genomes, and are capable of using a broad variety of electron acceptors for respiratory growth that include but are not limited to organohalides. This group comprises phylogenetically diverse proteobacterial OHRB such as members of the genera Geobacter, Desulfuromonas, Anaeromyxobacter, Desulfomonile, Desulfovibrio and Sulfurospirillum, and also the Desulfitobacterium spp. belonging to the Firmicutes $[13,46]$.

The key enzymes of OHR are reductive dehalogenases (RDase). For several of these enzymes the catalytic subunits have been purified and biochemically characterized, which are referred to as RDase catalytic subunit (as opposed to RdhA if only predicted based on sequence homology) [64, 78, 101, 255-259]. These studies, combined with PCR-based [64, 99-101, 260] and genomic analyses [20,92, 261], have revealed a conserved operon structure for RDase- 
encoding genes that consist of $r d h A$, coding for the catalytic subunit RdhA; $r d h B$, coding for a small putative membrane anchor ( $\mathrm{RdhB}$ ) that (presumably) locates the A subunit to the outside of the cytoplasmic membrane; and a variable set of accessory genes, the majority of which has been predicted to code for proteins involved in regulation and maturation of Rdh synthesis [46]. In general, the catalytic subunits (RdhAs) are characterized by two iron sulfurclusters and a cobamide (corrinoid cofactors) as cofactors in the active holoenzyme. As an exception to this rule, the chlorobenzoate RDase of Desulfomonile tiedjei contains a heme cofactor [255], and Sulfospirillum multivorans was shown to specifically require "norpseudo$\mathrm{B}_{12}$ " as the corrinoid cofactor [145]. Another conserved feature among the catalytic subunits is the presence of an $\mathrm{N}$-terminal Tat (twin-arginine translocation) signal peptide. After cofactor incorporation and folding of the cytoplasmic precursors of an RdhA enzyme, the Tat signal peptide is necessary for secretion of the mature protein through the cell membrane where it is located at the exocytoplasmic face of the cytoplasmic membrane [262].

\section{Phylogenetic context of $\operatorname{rdhA}$}

The genomes of OHRB encode either few (1-3) or many (10-40) distinct RDase gene operons. In general, larger numbers of $r d h A$ genes are encoded on genomes of obligate OHRB, with fewer present on genomes of microorganisms with versatile metabolism [13]. The expansion of the gene family through gene duplication has led to multiple non-identical $r d h A$ genes per genome and across diverse, unrelated genera resulting in a complicated evolutionary history for $r d h A$. A tree of RdhA protein sequences from known microorganisms (not including sequences retrieved from environmental samples) shows limited correlation between microorganism taxonomy and RDase phylogeny (Figure 3.1). Most phyla with multiple OHRB do not form monophyletic clades on the RdhA tree, and, even within clades comprised of a single phylum or group, the branching order of the RdhA does not usually follow the corresponding 16S rRNA-based taxonomy tree. In addition, microorganisms within the same genus often do not have the same RdhA homologs in their genomes. Taken together, the evolutionary relationships between the $r d h A$ genes can only be explained using a combination of vertical inheritance, gene duplication, and lateral gene transfer [46].

Most interesting from a functional perspective is the placement of the biochemically characterized RDases, which are distributed across the tree with little apparent relationship between proteins with shared substrate specificities when they are present in different phyla. The five characterized tetrachloroethene-reducing enzymes (PceA) are located in four distinct clades (Figure 3.1), indicating their low sequence similarity despite shared specificity. In 


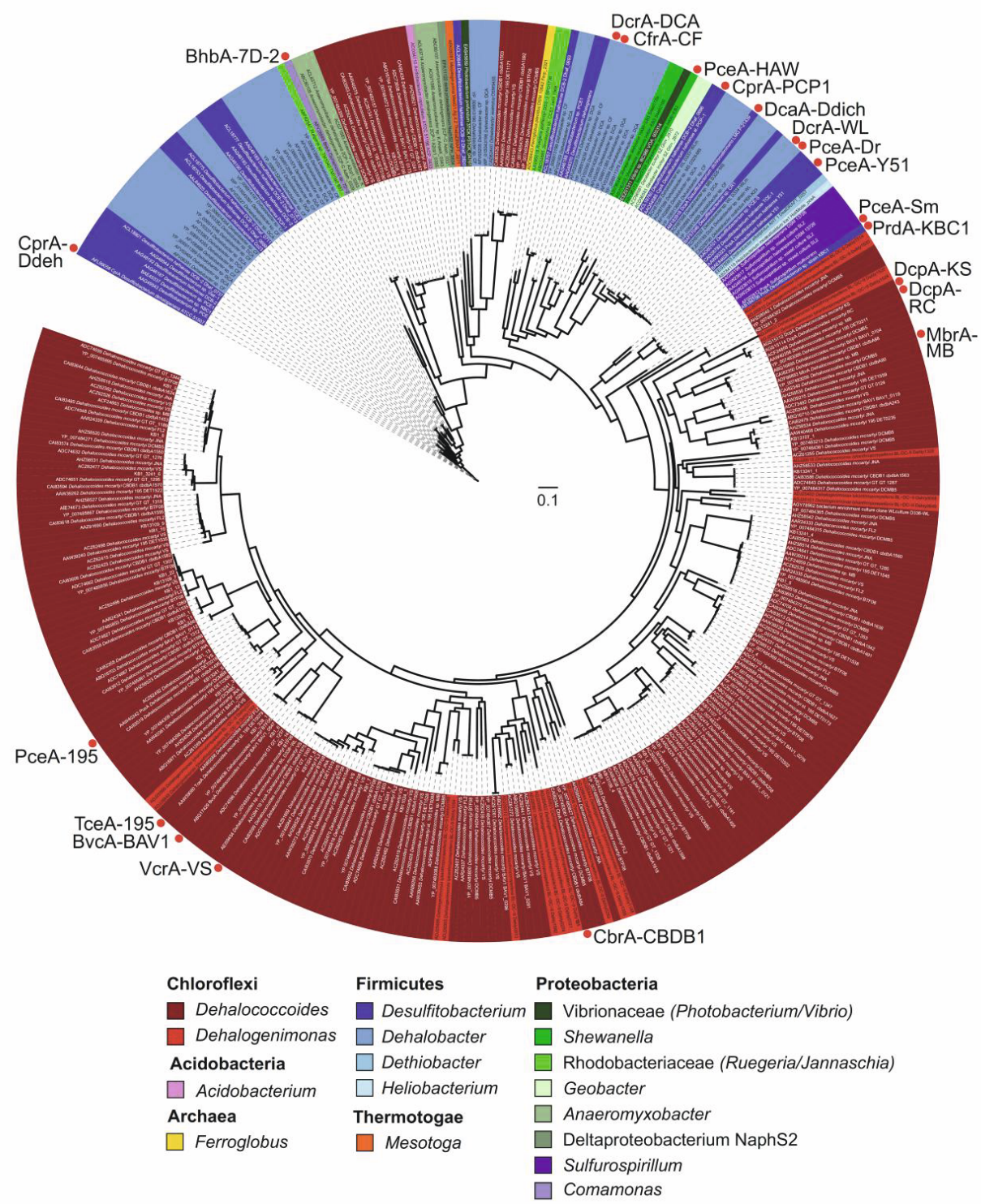

Figure 3.1 Maximum likelihood tree of RdhA amino acid sequences from known organisms. RdhAs are coloured by genus affiliation of the source organism or, in two cases, order affiliation. Functionally characterized RdhA are highlighted with red circles and named according to Table 3.1. The tree was constructed from 354 full-length RdhA sequences. Sequences were aligned using MUSCLE [263], and the alignment was iteratively refined using HMMER 3.0 [264] for ten iterations. Alignment columns were removed if they contained $>90 \%$ gaps, and the ends of the alignment trimmed to remove trailing ends. The tree was generated using RAxML $[265,266]$ under the PROTGAMMAWAG model of evolution and visualized using iTOL $[267,268]$. Microorganism information and protein NCBI accession numbers are included in each RdhA gene name on the tree. 
contrast, dichloroethane RDase DcrA and the chloroform RDase CfrA from Dehalobacter strains DCA and CF, respectively, share extremely high sequence identity (98\%) but do not have overlapping substrate specificities (Figure 3.1) [269]. The lack of correlation between sequence similarity and substrate specificity hinders the ability to predict substrates for novel genes, as well as to design primers targeting specific functions of interest.

\section{Molecular characterisation of RDase encoding genes}

Initial identification of $r d h$ gene sequences was performed by classical reverse genetics approaches based on $\mathrm{N}$-terminal amino acid sequences obtained from purified catalytic subunits [78, 260]. Subsequent availability of RDase encoding gene sequences has made it possible to design degenerate primers for PCR-based $r d h$ sequence retrieval. This has been particularly helpful for strains of $D$. mccartyi whose low biomass yields have hindered

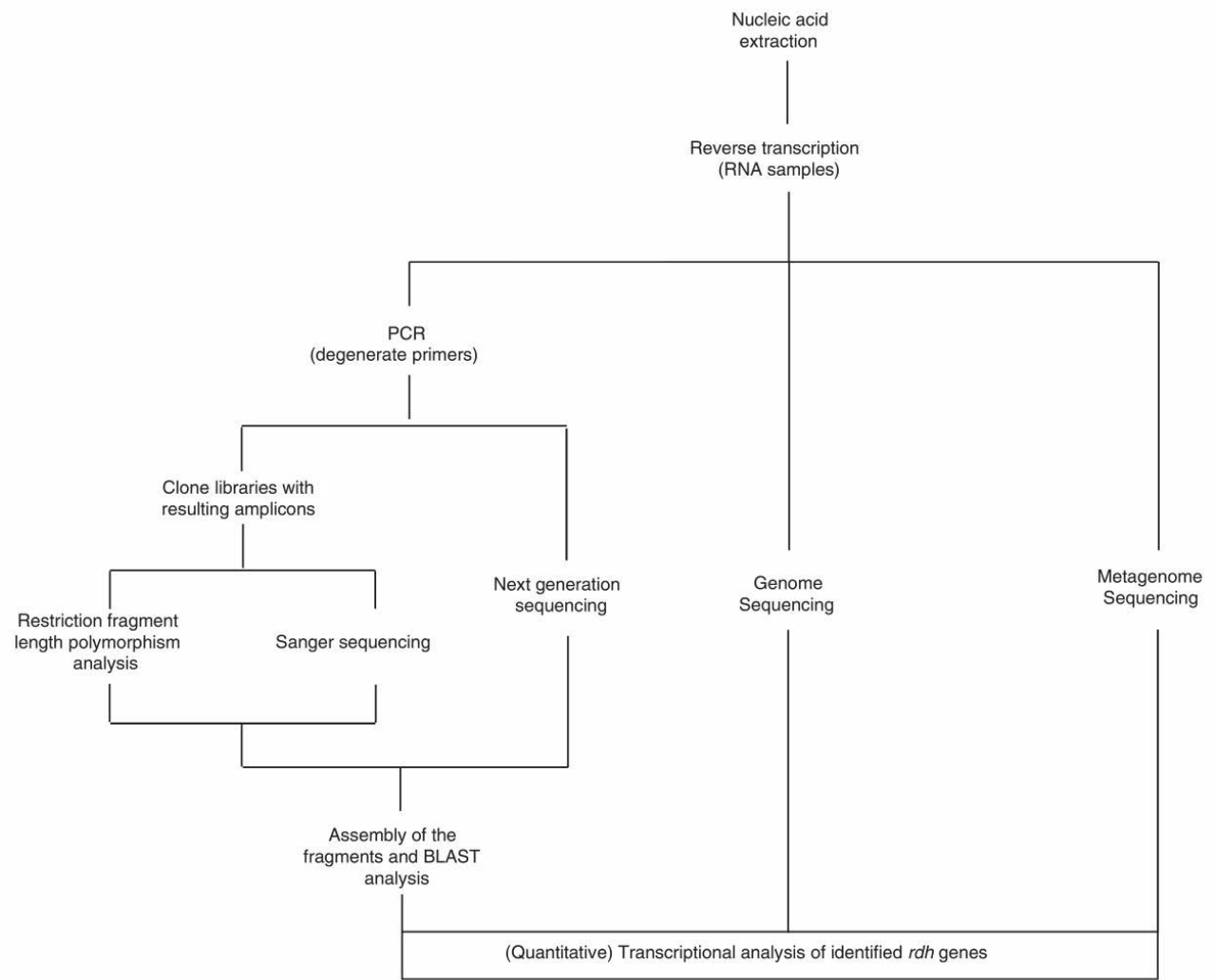

Figure 3.2 Overview of the methods used for molecular characterisation of RDase-encoding genes. 
protein-based identification in the past. In recent years genomic- and metagenomic-based approaches have also been applied for sequence-based retrieval and characterization of $r d h$ genes (Figure 3.2).

\section{Degenerate primers for characterization of RDase genes}

Degenerate primers are primer mixes with degenerate positions instead of a single sequence with specified bases. The primer degeneracy allows amplification of multiple loci simultaneously from different microorganisms, targeting sequences with similar but not identical motifs. Degenerate primers are widely used to amplify $r d h A$ genes, especially when the aim is to amplify genes from uncultivated OHRB for which genomic information is not available. Degenerate primers are usually designed based on conserved regions of RDase catalytic subunit encoding genes by aligning amino acids sequences found in sequence databases like GenBank [270], and using specific approaches including ConsensusDegenerate Hybrid Oligonucleotide Primer (CDHOP) [271]. The first set of degenerate primers (primer pair 1, Table 3.2) for an RDase gene was designed based on internal peptides in 1998, targeting a 1200 bp-long region of the PCE dehalogenase from Dehalospirillum multivorans [260] (later reclassified as Sulfurospirillum multivorans [142] (Table 3.2). In the following years, with increased availability of homologous sequences of RDase encoding genes, multiple degenerate primer sets were designed and applied for $r d h$ detection, mostly targeting conserved sequences of $r d h A[64,79,98,101,258]$ (Figure 3.3, Table 3.2). von Wintzingerode, Schlötelburg [272] developed primers (primer pair 13-16, Table 3.2) binding to sequence motifs encoding the conserved regions of PceA (Sulfurospirillum multivorans) and CprA (Desulfitobacterium dehalogenans) (Figure 3.3), which enabled amplification of cprA-like gene fragments from Desulfitobacterium hafniense, Desulfitobacterium sp. strain PCE1, and Dehalobacter restrictus. Expanding this technique further, Smidt [273] designed multiple sets of highly degenerate oligonucleotide primers (primer pairs 17-20, Table 3.2) based on twin-arginine signal peptides, iron-sulfur clusters, and five additional highly conserved sequence motifs (Figure 3.3), which were used to target $r d h A$ genes from different ortho-chlorophenol- and chloroethene-dechlorinating OHRB. Of all primers, the RRF2 and B1R primers (primer pair 4, Table 3.2) became one of the most popular sets [100, 274]. Originally used to identify the $b v c A B$ gene from $D$. mccartyi BAV1, this primer set targets the RRXFXK motif of the Tat signal peptide (RRF2) (Figure 3.3), and the WYEW motif internal to the downstream associated $r d h B$ gene (B1R), yielding amplicons of 1.5-1.7 bp containing almost complete rdhA genes (Table 3.2). Using this primer set, Krajmalnik-Brown [100] 
Tat signal peptide

(RR)
Iron sulfur clusters

(FeS1, FeS2)

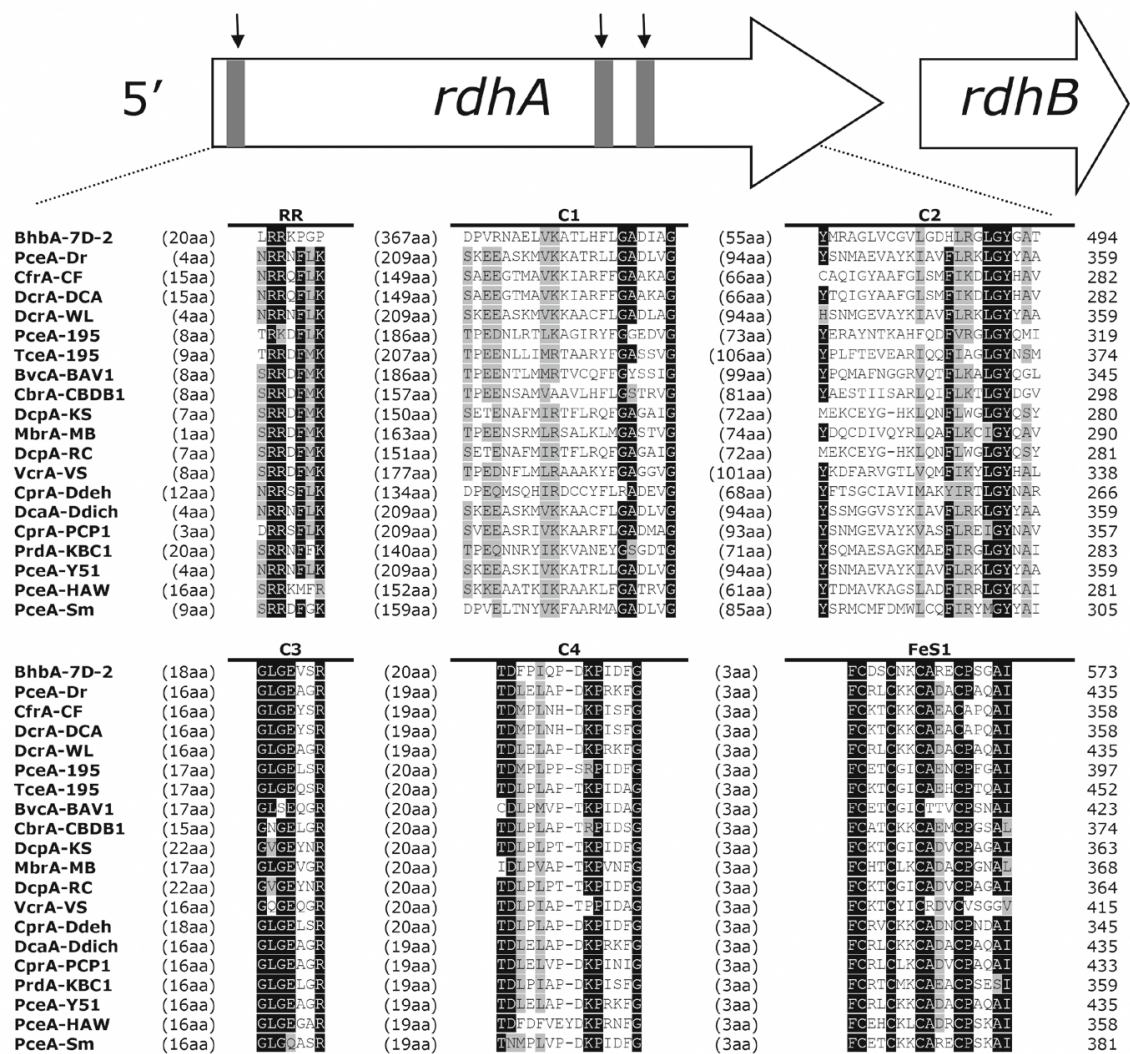

BhbA-7D-2

PceA-Dr
CfrA-CF

CfrA-CF

DCrA-DCA

DCrA-WL

PCeA-195

TCeA-195

CbrA-CBDB1

DCpA-KS

MbrA-MB

DCpA-RC

VcrA-VS

CprA-Ddeh

DcaA-Ddich

CprA-PCP1

PrdA-KBC

PCEA-Y51

PCEA-HAW

(19aa)

(3aa)

494
359

82

59
319

45

80

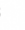

381

PceA-Sm

$(16 a a)$
$(27 a a)$
$(24 a a)$
$(24 a a)$
$(27 a a)$
$(23 a a)$
$(23 a a)$
$(23 a a)$
$(24 a a)$
$(29 a a)$
$(32 a a)$
$(29 a)$
$(24 a a)$
$(17 a a)$
$(27 a a)$
$(27 a a)$
$(24 a a)$
$(27 a a)$
$(33 a a)$
$(23 a a)$

Fes2

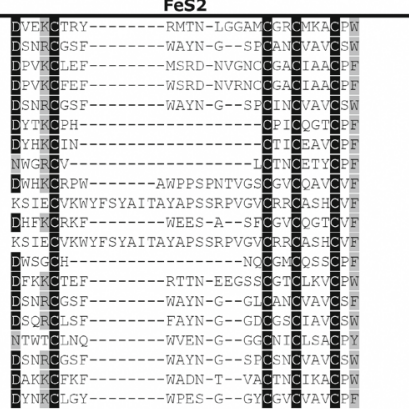

$(27 a a)$
$(26 a a)$
$(26 a)$
$(26 a a)$
$(26 a a)$
$(28 a a)$
$(29 a)$
$(29 a)$
$(28 a)$
$(28 a a)$
$(28 a a)$
$(28 a a)$
$(31 a a)$
$(28 a a)$
$(26 a a)$
$(26 a a)$
$(27 a a)$
$(26 a a)$
$(28 a a)$
$(27 a a)$

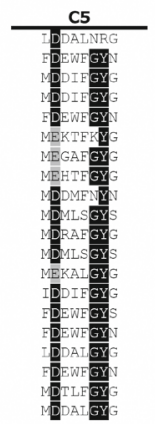

$\begin{array}{cc}\text { (420aa) } & 1071 \\ (30 a a) & 551 \\ (13 a a) & 456 \\ (13 a a) & 456 \\ (30 a a) & 551 \\ (22 a a) & 495 \\ (25 a a) & 554 \\ (16 a a) & 516 \\ (25 a a) & 488 \\ (19 a a) & 483 \\ (27 a a) & 488 \\ (19 a a) & 484 \\ (23 a a) & 519 \\ (22 a a) & 447 \\ (30 a a) & 551 \\ (29 a a) & 548 \\ (20 a a) & 463 \\ (30 a a) & 551 \\ (16 a a) & 468 \\ (37 a a) & 501\end{array}$

Figure 3.3 Physical map of $\operatorname{rdh} A B$ operon (top) and primary sequence alignment of known RdhAs (bottom). Note that in some cases, the order of genes in the operon is reversed (i.e. $\operatorname{rdh} B A$, rather than $\operatorname{rdh} A B$ ). Clustal $\mathrm{W}$ [275] multiple sequence alignment was conducted by using BioEdit version 7.2.5 (http://bioedit.software.informer.com/). The conserved sequence motifs among experimentally characterized reductive dehalogenases (RR, two Fe-S and $\mathrm{C} 1-\mathrm{C} 5$ ) 
are indicated with black backgrounds. Functionally characterized RdhAs are named according to Table 3.1.

Table 3.1 Functionally characterized RDase catalytic subunits.

\begin{tabular}{|l|l|l|l|l|}
\hline Code & Protein & Microorganism & $\begin{array}{l}\text { Protein NCBI } \\
\text { accession } \\
\text { numbers }\end{array}$ & Reference \\
\hline BhbA-7D-2 & BhbA & Comamonas sp. 7D-2 & AFV28965.1 & {$[151]$} \\
\hline PceA-Dr & PceA & Dehalobacter restrictus PER-K23 & CAD28790.2 & {$[64]$} \\
\hline CfrA-CF & CfrA & Dehalobacter sp. strain CF & AFQ20272.1 & {$[269]$} \\
\hline DcrA-DCA & DcrA & Dehalobacter sp. strain DCA & AFQ20273.1 & {$[269]$} \\
\hline DcrA-WL & DcrA & Dehalobacter sp. strain WL & ACH87594.1 & {$[276]$} \\
\hline PceA-195 & PceA & Dehalococcoides mccartyi 195 & AAW40342.1 & {$[97]$} \\
\hline TceA-195 & TceA & Dehalococcoides mccartyi 195 & AAF73916.1 & {$[98]$} \\
\hline CbrA-CBDB1 & CbrA & Dehalococcoides mccartyi CBDB1 & CAI82345.1 & {$[103]$} \\
\hline DcpA-KS & DcpA & Dehalococcoides mccartyi KS & AGS15112.1 & {$[104]$} \\
\hline MbrA-MB & MbrA & Dehalococcoides mccartyi MB & ADF96893.1 & {$[99]$} \\
\hline DcpA-RC & CbrA & Dehalococcoides mccartyi RC & AGS15114.1 & {$[104]$} \\
\hline VcrA-VS & VcrA & Dehalococcoides mccartyi VS & AAQ94119.1 & {$[101]$} \\
\hline CprA-Ddeh & CprA & Desulfitobacterium dehalogenans JW/IU-DC1 & AAD44542.1 & {$[78,97]$} \\
\hline DcaA-Ddich & DcaA & Desulfitobacterium dichloroeliminans DCA1 & CAJ75430.1 & {$[83]$} \\
\hline CprA-PCP1 & CprA & Desulfitobacterium hafniense sp. PCP-1 & AAQ54585.2 & {$[77]$} \\
\hline PrdA-KBC1 & PrdA & Desulfitobacterium sp. strain KBC1 & BAE45338.1 & {$[80]$} \\
\hline PceA-Y51 & PceA & Desulfitobacterium sp. strain Y51 & AAW80323.1 & {$[79]$} \\
\hline PceA-HAW & PceA & Shewanella sediminis HAW-EB3 & ABV38373.1 & {$[277]$} \\
\hline PceA-Sm & PceA & Sulfurospirillum multivorans DSM 12446 & AAC60788.1 & {$[260]$} \\
\hline
\end{tabular}

found seven $r d h A$ genes in D. mccartyi BAV1. Later, the RR2F/B1R pair was widely used to detect $r d h A$ 's from $D$. mccartyi isolates and enrichments, including $13 r d h$ loci in strain CBDB1 [274], 14 in strain FL2, 14 in the mixed culture KB1 [278], 8 in the enrichment culture TUT2264 [279] and 4 from TCEcontaminated groundwater samples [194]. Although the RR2F/B1R primer set was designed based on $17 \mathrm{rdh} A B$ genes in the genome of D. mccartyi 195 [100], the primer set fails to amplify tceA-like genes [99]. Furthermore, it should be noted that the $r d h-A$ and $-B$ genes are not always oriented in an A-B direction in the $r d h$ operon [96]. To circumvent this shortcoming, Chow [99] designed a 
complimentary primer set RDH F1C/RDH R1C (primer pair 7, Table 3.2) targeting the coding region of the twin arginine motif and conserved PIDDG motif, respectively (Figure 3.3, Table 3.2). With a product size of $1200 \mathrm{bp}$, this primer pair targeted only the catabolic subunit gene $(r d h A)$, and (at the time of design) could cover $\sim 90 \%$ of the known $r d h A s$ in D. mccartyi populations. Using this primer set, the authors detected seven putative $r d h A$ genes from $D$. mccartyi strain MB [99]. Another potential problem with the RR2F/B1R primer pair is that, although the N-terminal Tat signal peptide motif is highly conserved among RdhAs, it is also found in numerous other exported proteins, and as such is not unique to this class of proteins [262]. Meanwhile, Regeard [271] had designed seven degenerate primers (primer pairs 23-28, Table 3.2) based on four conserved regions of chloroethene RDase catalytic subunit encoding amino acid sequences from Desulfitobacterium sp. PCE-1, S. multivorans and D. restrictus, and chlorophenol RDase catalytic subunit encoding amino acid sequences from Desulfitobacterium dehalogenans, D. hafniense strain DCB-2 and Desulfitobacterium chlororespirans (Table 3.2). This suite of degenerate primers did not utilize the Tat signal sequence, reducing non-specific amplification, but also resulted in shorter amplicons than the primers developed by Chow [99]. The primers were originally used to estimate the diversity of $r d h A$ from tetra- and trichloroethene dechlorinating enrichments [271] and later to detect $r d h A$ from Desulfitobacterium dichloroeliminans DCA1 [83, 280], Dehalobacter sp. WL [276] and sub-seafloor sediments[178].

It is evident from the alignment of $r d h A$ genes that one primer pair cannot cover all possible sequences. Accordingly, Futagami [178] used an array of degenerate primer sets designed to target different $r d h A s$ [100, 271, 272, 274]. Using this approach, 32 putative $r d h A$ phylotypes were detected from marine subsurface sediments [178]. In a further step, Wagner et al. (2009) designed 13 degenerate primer pairs to monitor the expression of all $32 \mathrm{rdh} A$ genes present in the genome of D. mccartyi CBDB1 [281]. Although ideal in order to cover a diversity of different $r d h A s$, application of such an array of degenerate primers would dramatically increase the work-load in subsequent clone library construction. Besides, most of the degenerate primers were designed based on the limited number of RDase sequences available at the time, which could potentially overlook distantly related novel $r d h A s$. To adequately cover the full diversity of $r d h A$ homologous sequences displaying lower similarity, a suite of 


\begin{tabular}{|c|c|c|c|c|c|c|c|c|c|c|c|c|c|}
\hline 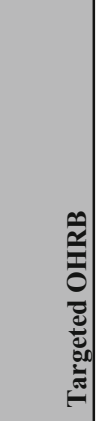 & 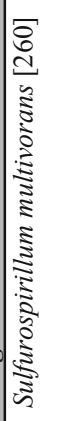 & & 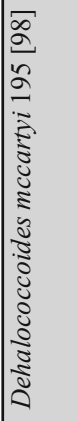 & & 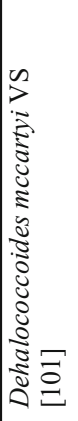 & & 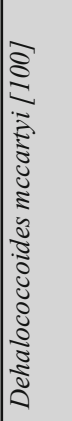 & 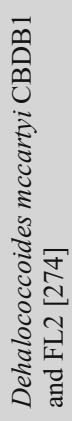 & 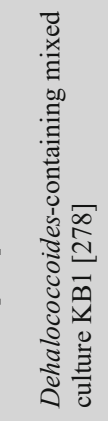 & 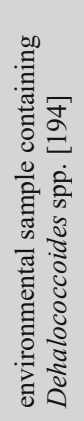 & 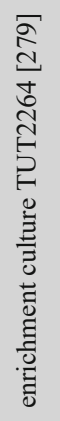 & 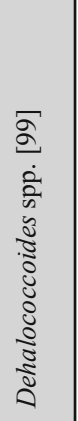 & 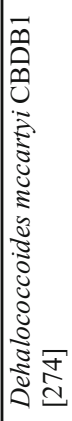 \\
\hline के है & 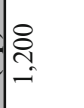 & & $\overline{\vec{q}}$ & & & & $\begin{array}{l}8 \\
0 \\
n \\
-1\end{array}$ & 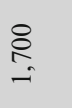 & $\frac{8}{i}$ & $\frac{8}{i}$ & $\begin{array}{c}\stackrel{N}{n} \\
\stackrel{n}{\wedge}\end{array}$ & \begin{tabular}{l}
1 \\
8 \\
0 \\
\hdashline
\end{tabular} & in \\
\hline 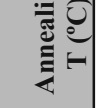 & fo & & 8 & & F & & $\stackrel{\infty}{+}$ & $\stackrel{\infty}{+}$ & $\stackrel{\infty}{+}$ & $\stackrel{\infty}{+}$ & ' & $\stackrel{\infty}{+}$ & |' \\
\hline 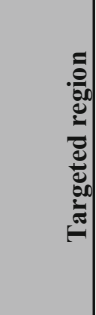 & 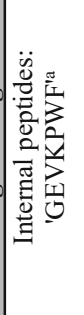 & 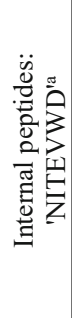 & 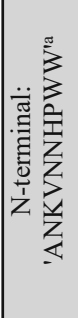 & 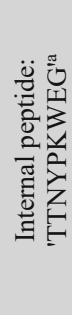 & 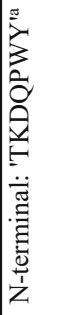 & 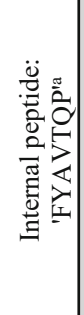 & 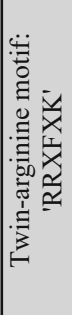 & & 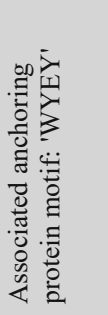 & & & & 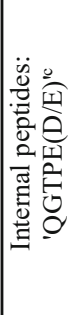 \\
\hline 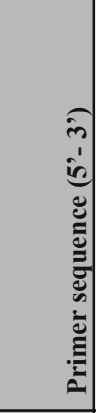 & 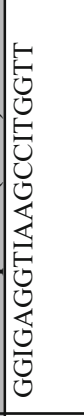 & 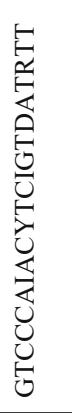 & 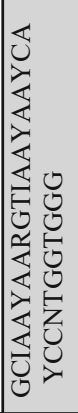 & 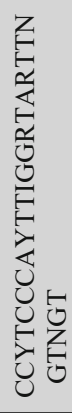 & 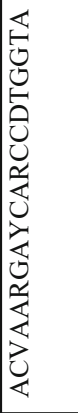 & 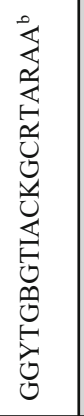 & 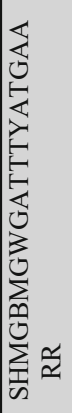 & & 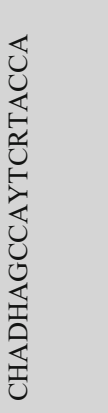 & & & & 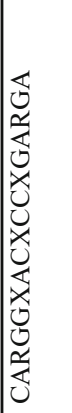 \\
\hline 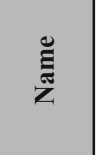 & 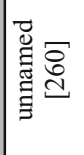 & 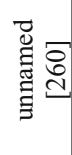 & ${ }_{\substack{\alpha\\
}}^{\infty}$ & 곱 & 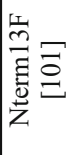 & 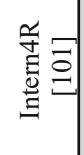 & 我孚 & & $\cong$ & & & & 烝 \\
\hline 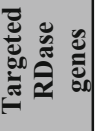 & ¿ूँ & & I & & $\underset{\Xi}{\pi}$ & & $\begin{array}{l}5 \\
\vdots \\
\vdots\end{array}$ & & $\begin{array}{l}0 \\
\vdots \\
\vdots\end{array}$ & & & & $\underset{\mathbb{Z}}{\mathbb{Z}}$ \\
\hline 离 & - & & N & & $m$ & & t & & & & & & in \\
\hline
\end{tabular}




\begin{tabular}{|c|c|c|c|c|c|c|c|c|c|c|c|c|}
\hline & 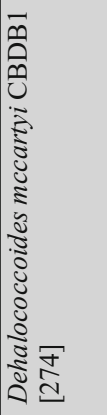 & & 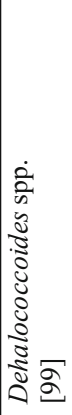 & & 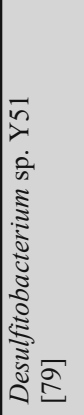 & & \multicolumn{2}{|c|}{ 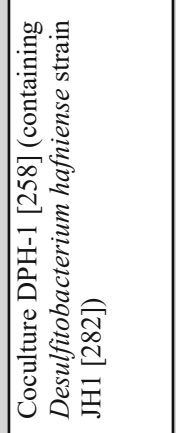 } & 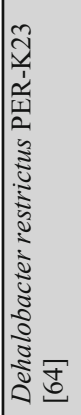 & & \multicolumn{2}{|l|}{ 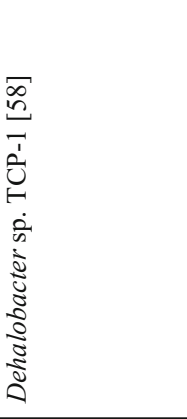 } \\
\hline & $\frac{8}{2}$ & & 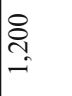 & & 8 & & $\infty$ & & 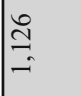 & & $\frac{8}{\stackrel{8}{i}}$ & \\
\hline & ' & & f & & \& & & $\mathscr{F}$ & & in & & ' & \\
\hline 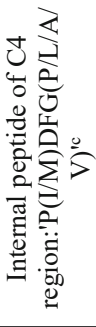 & 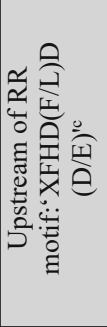 & 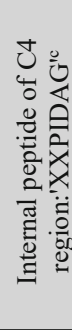 & 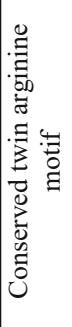 & 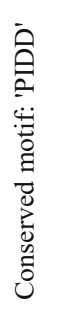 & 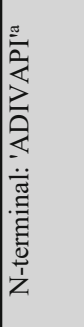 & 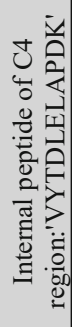 & 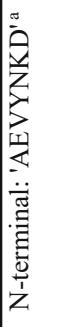 & 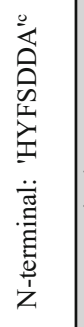 & 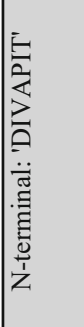 & 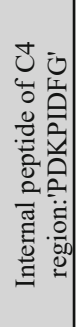 & 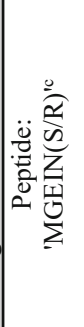 & 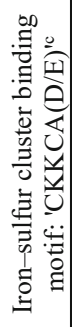 \\
\hline 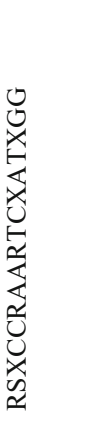 & 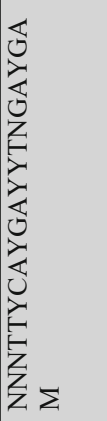 & 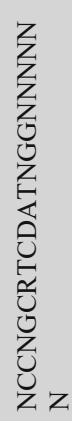 & 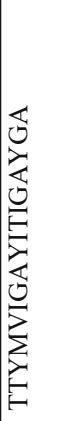 & 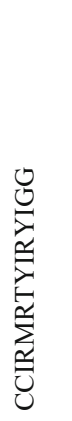 & 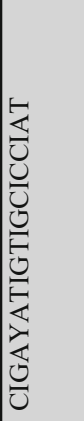 & 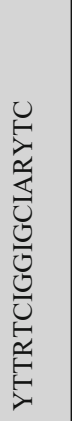 & 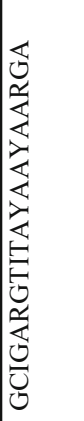 & 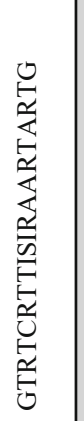 & 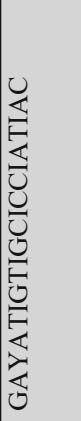 & 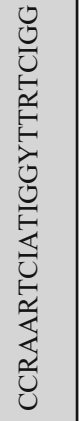 & 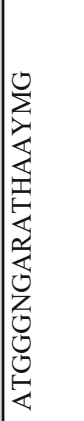 & 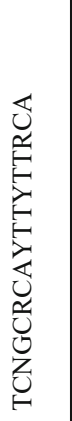 \\
\hline 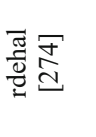 & 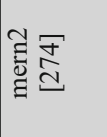 & 气 & 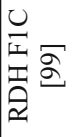 & 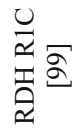 & 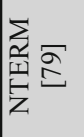 & 吉只 & 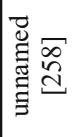 & 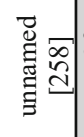 & 峁 & 守吉 & 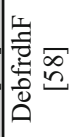 & 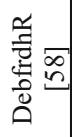 \\
\hline & $\underset{\mathbb{Z}}{\mathbb{Z}}$ & & $\underset{\Xi}{\Xi}$ & & 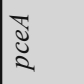 & & $\begin{array}{l}U \\
\Xi \\
\Xi\end{array}$ & & $\begin{array}{l}\mathbb{Z} \\
\stackrel{\Omega}{2}\end{array}$ & & 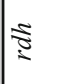 & \\
\hline & 0 & & $r$ & & $\infty$ & & a & & $\stackrel{\varrho}{0}$ & & $\Xi$ & \\
\hline
\end{tabular}




\begin{tabular}{|c|c|c|c|c|c|c|c|c|c|c|c|}
\hline 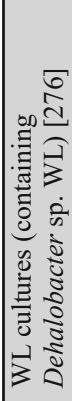 & & \multicolumn{2}{|c|}{ 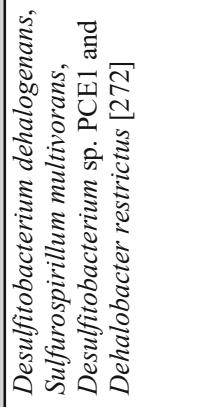 } & \multicolumn{2}{|l|}{ 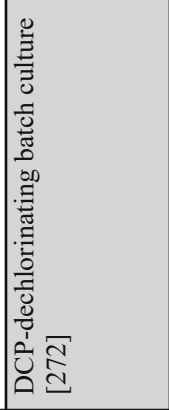 } & \multicolumn{2}{|c|}{ 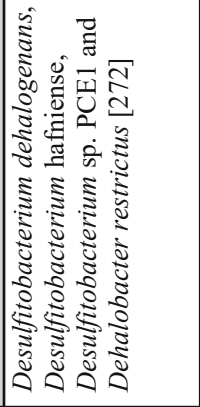 } & \multicolumn{2}{|c|}{ 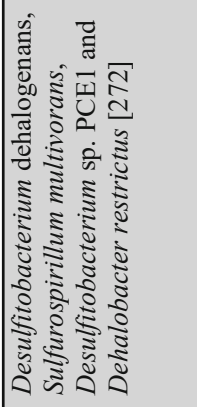 } & \multicolumn{2}{|c|}{ 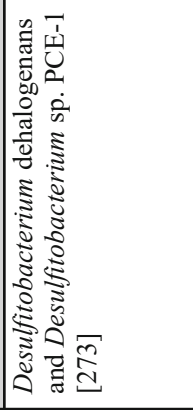 } \\
\hline$\stackrel{8}{8}$ & & : & & in & & $\stackrel{\text { }}{\gamma}$ & & $\begin{array}{l}8 \\
i \\
i\end{array}$ & & ' & \\
\hline$\stackrel{\infty}{+}$ & & in & & in & & in & & in & & $\begin{array}{l}\text { o } \\
\text { ín } \\
\text { in }\end{array}$ & \\
\hline 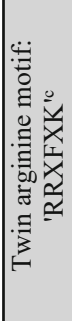 & 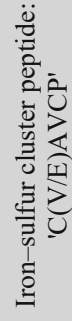 & 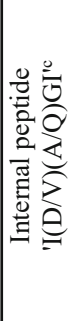 & 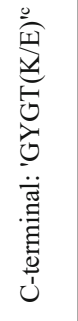 & 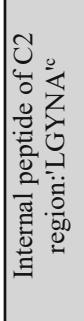 & 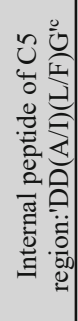 & 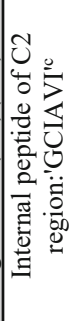 & 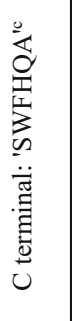 & 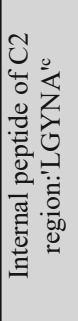 & 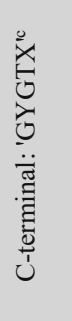 & 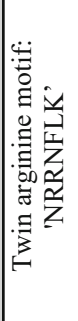 & 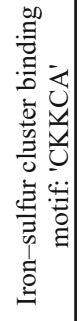 \\
\hline 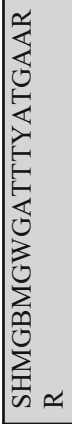 & 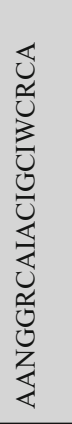 & 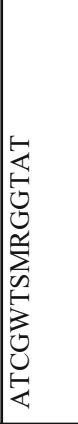 & 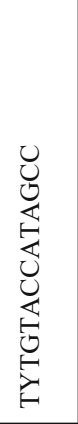 & 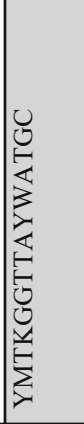 & 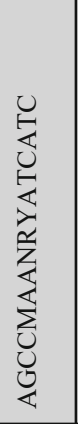 & 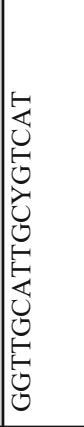 & 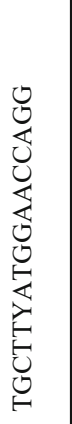 & 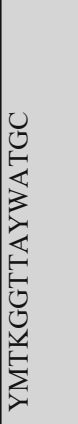 & 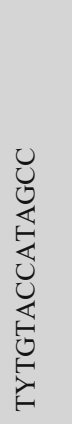 & 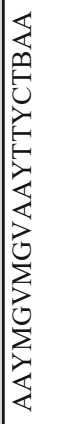 & 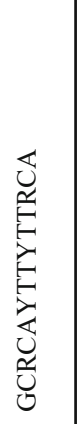 \\
\hline 赵 & ลิ촟 & 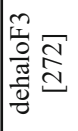 & 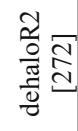 & 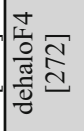 & $\begin{array}{l}\frac{\partial}{0} \\
\frac{N}{\pi} \\
\frac{\pi}{0} \\
\frac{N}{d}\end{array}$ & 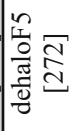 & $\begin{array}{l}\frac{d}{0} \\
\frac{\pi}{0} \\
\frac{\pi}{d} \\
\frac{\pi}{d}\end{array}$ & 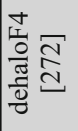 & 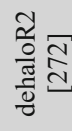 & 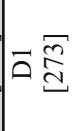 & 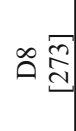 \\
\hline 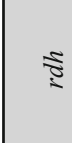 & & 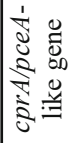 & & 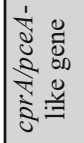 & & 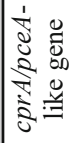 & & 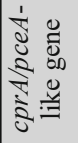 & & $\vec{s}$ & \\
\hline$\simeq$ & & $\because$ & & \pm & & $\cong$ & & $\because$ & & $\beth$ & \\
\hline
\end{tabular}




\begin{tabular}{|c|c|c|c|c|c|c|c|c|c|c|}
\hline \multicolumn{2}{|c|}{ 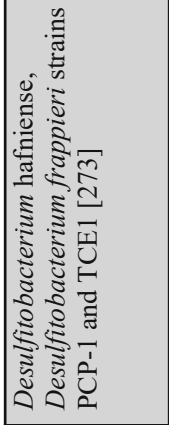 } & \multicolumn{2}{|c|}{ 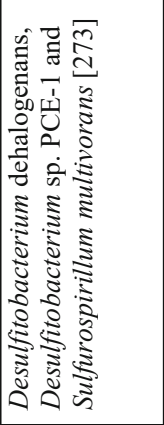 } & \multicolumn{2}{|c|}{ 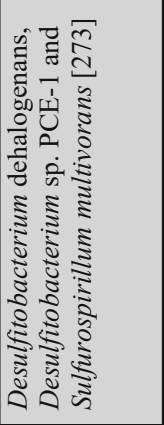 } & \multicolumn{2}{|l|}{ 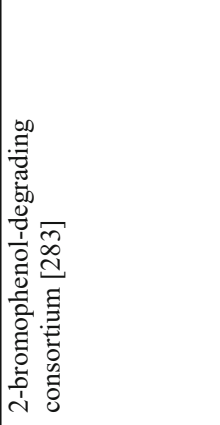 } & 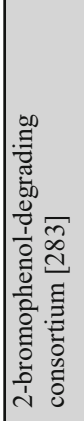 & 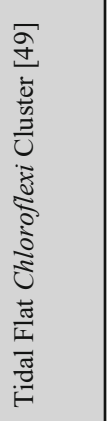 & 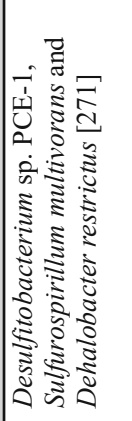 \\
\hline ' & & ' & & 1 & & $\stackrel{n}{\circ}$ & & 尽 & \& : & 횽 \\
\hline 怼 & & $\begin{array}{l}q \\
y \\
2 \\
m\end{array}$ & & ơ & & $n$ & & $\approx n$ & lo & $\mathscr{\gamma}$ \\
\hline 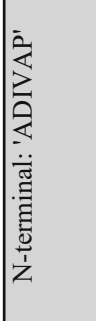 & 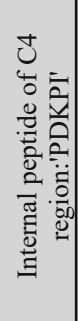 & 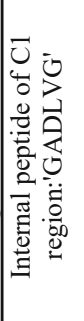 & 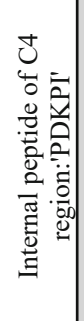 & 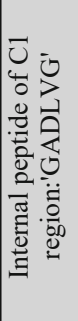 & 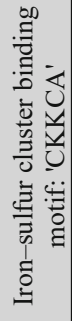 & 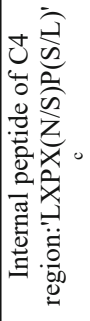 & 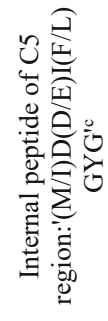 & 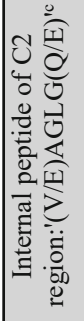 & 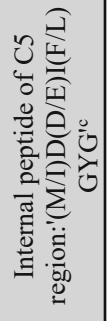 & 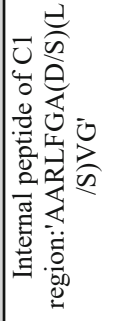 \\
\hline 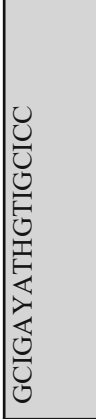 & 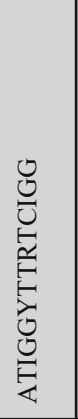 & 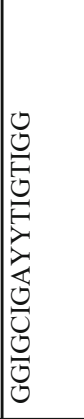 & 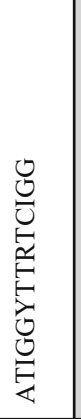 & 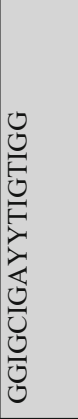 & 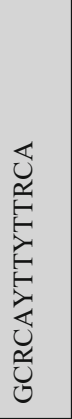 & 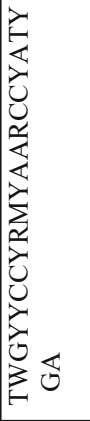 & 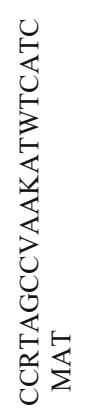 & 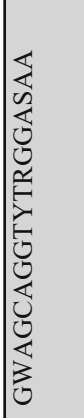 & 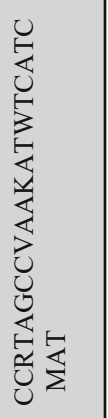 & 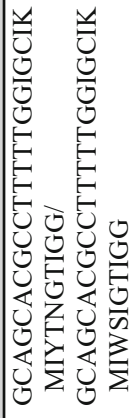 \\
\hline 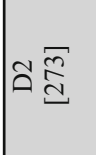 & $\hat{0} \stackrel{\bar{v}}{\hat{v}}$ & $+\stackrel{\sqrt[n]{\Delta}}{\stackrel{\Xi}{\Delta}}$ & 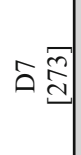 & 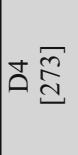 & 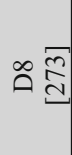 & 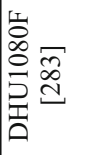 & 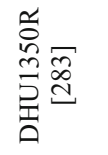 & 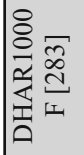 & 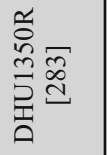 & 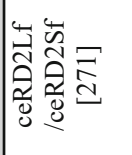 \\
\hline 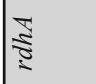 & & $\underset{\Xi}{\beth}$ & & 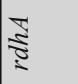 & & $\frac{\pi}{3}$ & & $\frac{\pi}{2}$ & & 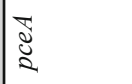 \\
\hline$\stackrel{\infty}{\infty}$ & & 2 & & 요 & & $\vec{\sim}$ & & तี & & $\ddot{\imath}$ \\
\hline
\end{tabular}




\begin{tabular}{|c|c|c|c|c|c|c|c|c|}
\hline 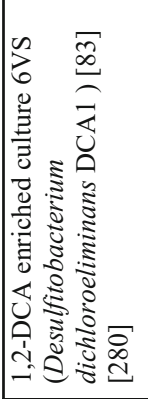 & 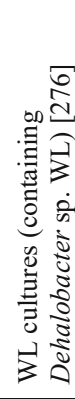 & 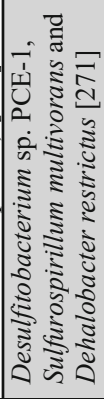 & & 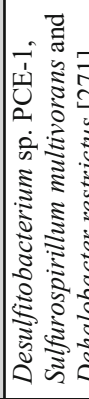 & & 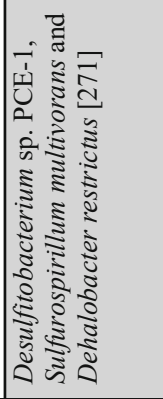 & & 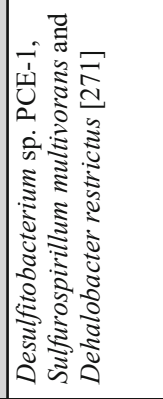 \\
\hline 至 & , & 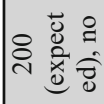 & & 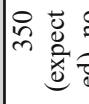 & & 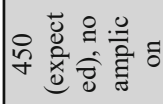 & & 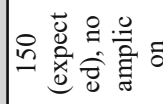 \\
\hline f & $\stackrel{\infty}{+}$ & f & & in & & in & & in \\
\hline 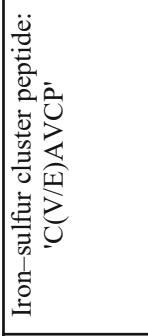 & & 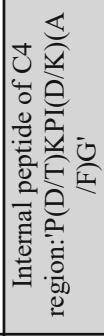 & 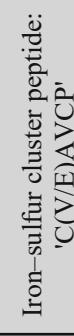 & 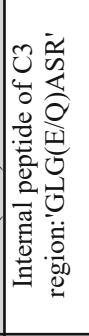 & 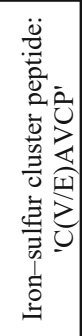 & 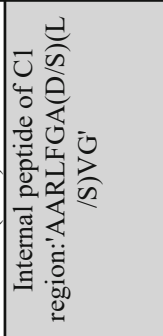 & 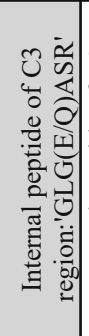 & 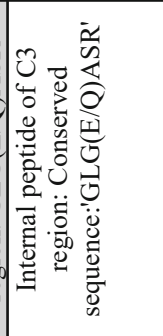 \\
\hline 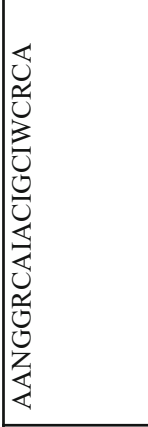 & & 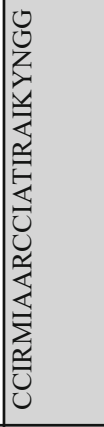 & 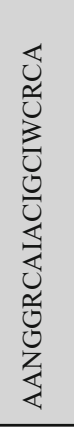 & 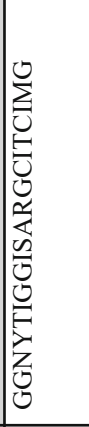 & 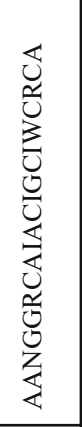 & 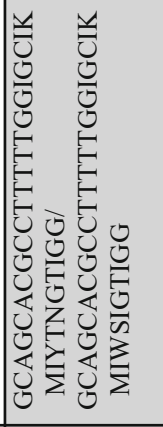 & $\begin{array}{l}0 \\
0 \\
z \\
0 \\
\vdots \\
0 \\
0 \\
0 \\
0 \\
0 \\
0 \\
0 \\
0 \\
0 \\
0\end{array}$ & 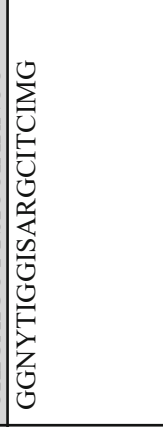 \\
\hline 容胥 & & 峞 & ลิ & 守突 & $\approx$ & 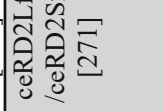 & 亲司 & 守空 \\
\hline & & $\begin{array}{l}\mathbb{Z} \\
\mathscr{Z}\end{array}$ & & $\begin{array}{l}\Xi \\
\Xi \\
\Xi\end{array}$ & & $\begin{array}{l}\Xi \\
\Xi \\
\Xi\end{array}$ & & Zू \\
\hline & & $\underset{\sim}{*}$ & & 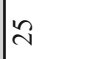 & & | & & $\hat{\imath}$ \\
\hline
\end{tabular}




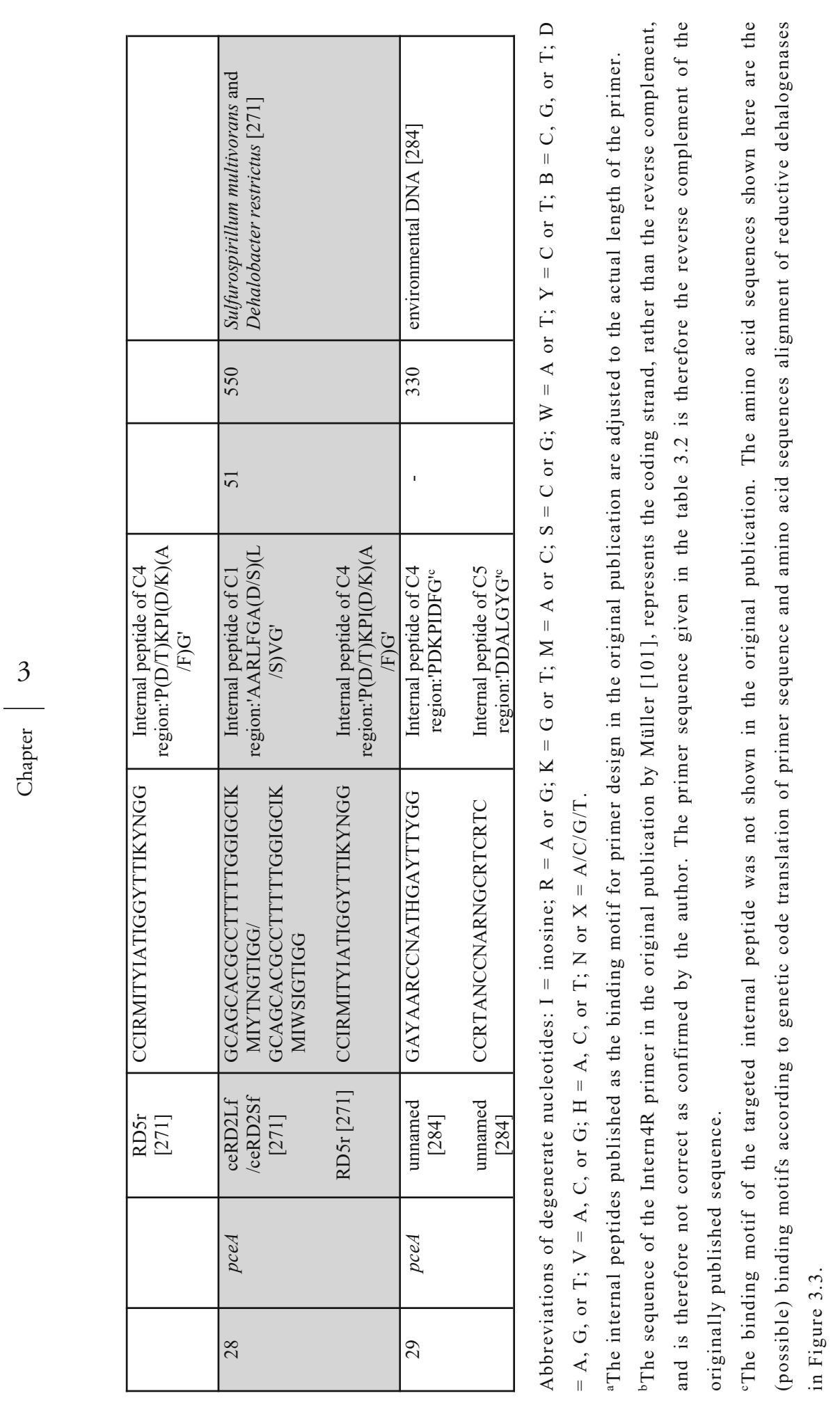


degenerate PCR primers (comprising 44 phylogeny-derived groups) targeting 255 RDase catalytic subunit genes was recently designed and applied to environmental and enrichment culture samples [285]. Subsequent application of Illumina HiSeq next-generation sequencing identified a much broader diversity of $r d h A$ gene sequence than was previously accounted for [285].

\section{Specific primers and application potential}

In addition to degenerate primers, a broad range of specific primers targeting selected RDase genes have been designed (Table 3.3). Usually, specific primers are developed and used for different purposes than the more exploratory degenerate primer sets, including analysis of gene expression in S. multivorans [260] and Desulfitobacterium sp. Y51 [79], preparation of probes for Southern blot hybridization [83], detection of Sulfurospirillum $r d h A$ genes by TRFLP [286], and detection of known chloroethene RDase genes [271]. Additionally, specific primers were used for putative $r d h A$ detection from various OHRB encoding tce $A$ [98], $b v c A$ [100], vcrA [101], cfrA [269], dcrA [269], and $d c p A$ [104](Table 3.3). Some primer sets have been designed to obtain complete $r d h A B$ sequences of specific reductive dehalogenases, including $p c e A B[64,260,273], v c r A B[101]$ and $\operatorname{cpr} A B[77,273]$.

To connect the presence of specific $r d h A$ s with OHR activity, multiple primer sets were developed for $r d h A$ quantification (Table 3.4). For instance, by using D. mccartyi-specific primer sets targeting $b v c A$ (BVC925F/1017R), vcrA (Vcr1022F/1093R), and tce A (TceA1270F/1336R), Ritalahti [195] were able to link abundance and identity of D. mccartyi populations to different organohalide electron acceptors. From this, the authors were able to predict the composition of a $D$. mccartyi community using the quantitative results of $r d h A$ and 16S rRNA gene-targeted quantitative PCR (qPCR). qPCR assays with $r d h A$ targeted primers have also been used for monitoring of bioremediation in polluted sites [83, 104, 195]. Building upon this knowledge, microfluidics-based, moderately to massively parallel qPCR approaches were recently developed for covering much of the known $r d h A$ sequence space. The method was helpful in quantitative analysis of $r d h A$ repertoires and identification of closely related populations of OHRB $[287,288]$. It should be noted that to date all known RDase and 16S rRNA genes identified in the genomes of known D. mccartyi strains occur as single copies (unlike Desulfitobacterium spp. and Dehalobacter spp. strains with multiple copies of $16 \mathrm{~S}$ rRNA genes), suggesting that qPCR data can be converted from gene copy 
$\frac{3}{\text { 苛 }}$

\begin{tabular}{|c|c|c|c|c|c|c|c|c|c|}
\hline 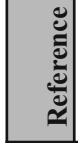 & $\begin{array}{l}0 \\
\text { D } \\
\text { ปे }\end{array}$ & 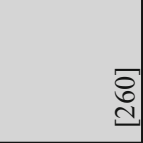 & $\stackrel{\infty}{\sigma}$ & $\stackrel{\vec{\imath}}{\vec{v}}$ & 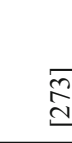 & 인 & t & $\overrightarrow{\mathrm{d}}$ & $\overrightarrow{\widetilde{N}}$ \\
\hline :ै & & & & & & & 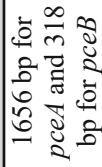 & & \\
\hline 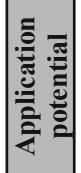 & 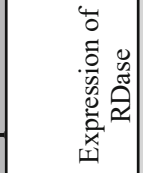 & 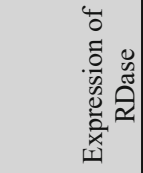 & 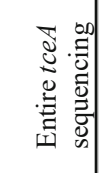 & 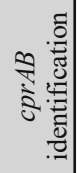 & 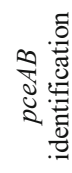 & 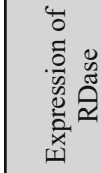 & 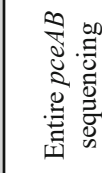 & 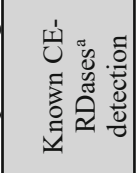 & 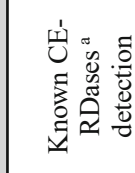 \\
\hline 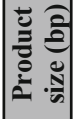 & & & హิ & $\begin{array}{l}+ \\
\infty \\
\infty \\
-1\end{array}$ & $\underset{\infty}{+}$ & $\stackrel{8}{?}$ & ' & $\frac{\infty}{6}$ & ळे \\
\hline 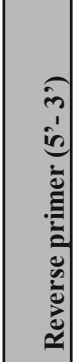 & 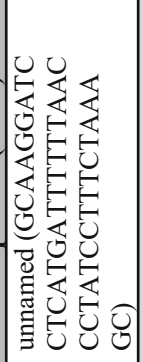 & 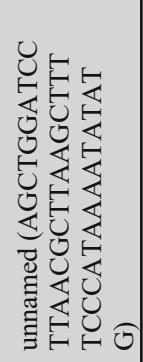 & 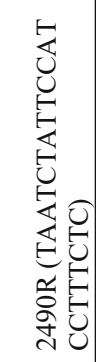 & 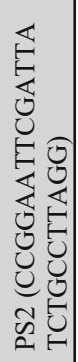 & 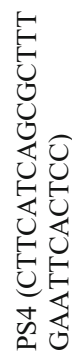 & 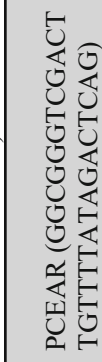 & 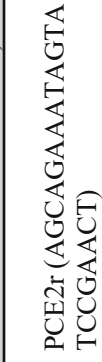 & 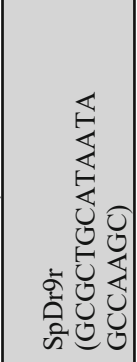 & 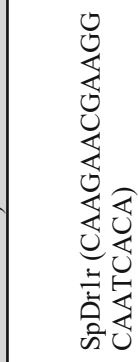 \\
\hline 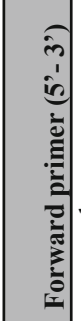 & 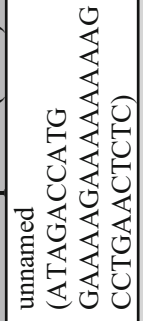 & 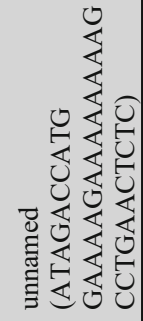 & 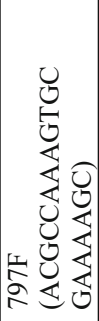 & 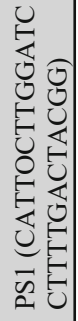 & 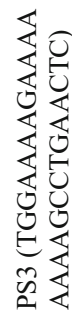 & 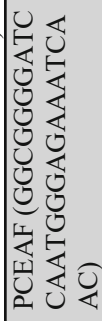 & 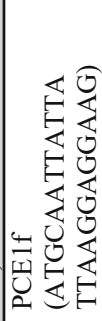 & 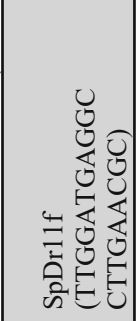 & 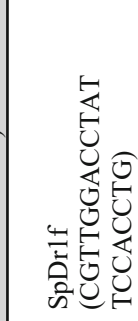 \\
\hline 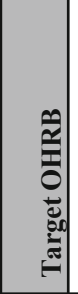 & 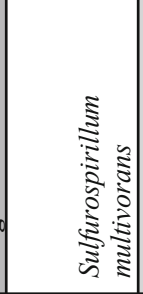 & 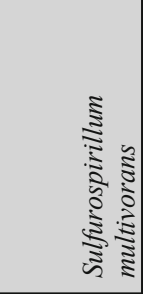 & 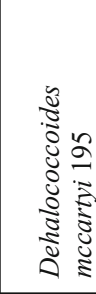 & 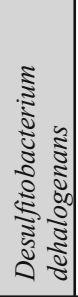 & 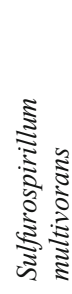 & 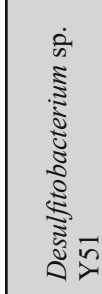 & 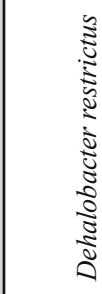 & 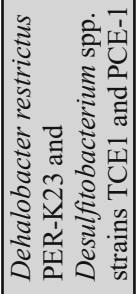 & 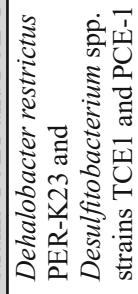 \\
\hline 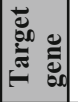 & : & $\check{\Omega}$ & $\approx$ & के & $\mathscr{J}$ & 2 & $\begin{array}{l}\stackrel{0}{\Xi} \\
\Xi \\
\Xi\end{array}$ & 2 & $\begin{array}{l}\Xi \\
\mathscr{8}\end{array}$ \\
\hline
\end{tabular}




\begin{tabular}{|c|c|c|c|c|c|c|c|c|c|}
\hline$\sqrt{3}$ & $\overrightarrow{\mathrm{N}}$ & $\overrightarrow{\mathrm{d}}$ & $\overrightarrow{\widehat{\Delta}}$ & 은 & 8 & 콘 & $\stackrel{\infty}{\triangleq}$ & $\tilde{\infty}$ & $\begin{array}{l}\text { D } \\
\stackrel{\sim}{\sim}\end{array}$ \\
\hline & & & & & 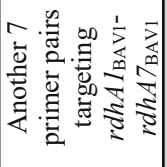 & & 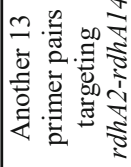 & & \\
\hline 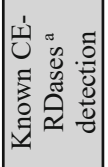 & 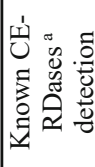 & 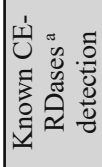 & 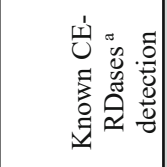 & 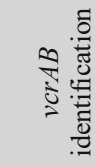 & 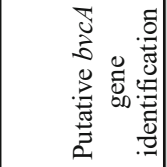 & 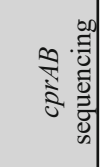 & 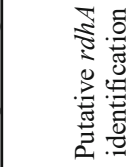 & 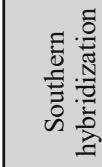 & $\frac{\vec{T}}{\frac{a}{1}}$ \\
\hline$\stackrel{+}{-}$ & $\stackrel{+}{\Xi}$ & ఏ & Әे & ' & ळે & $\begin{array}{l}8 \\
\text { r. } \\
\text { in }\end{array}$ & & $\underset{\sim}{\sim}$ & \\
\hline 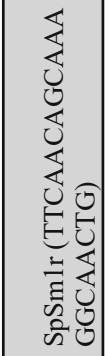 & 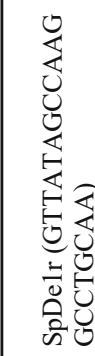 & 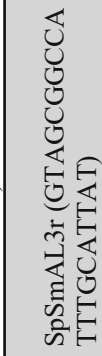 & 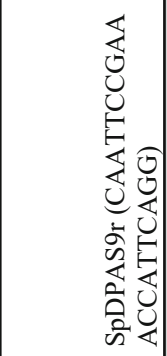 & 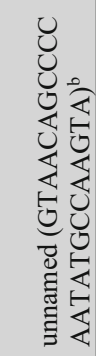 & 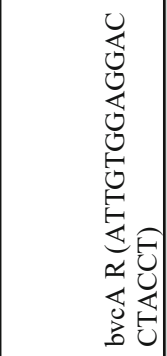 & 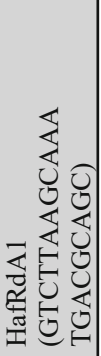 & 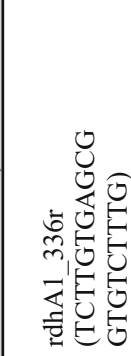 & 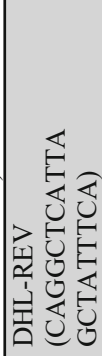 & 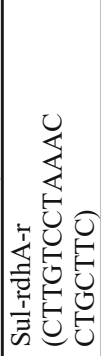 \\
\hline 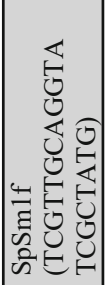 & 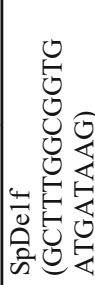 & 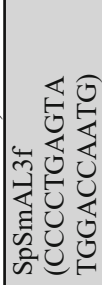 & 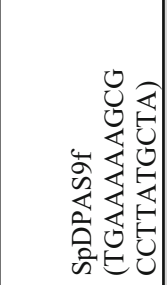 & 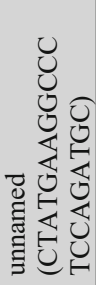 & 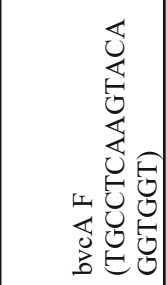 & 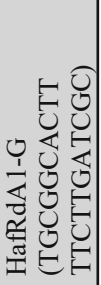 & 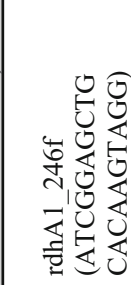 & 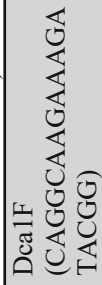 & 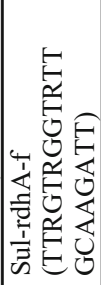 \\
\hline 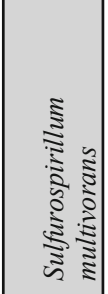 & 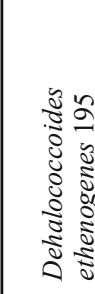 & 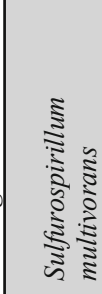 & 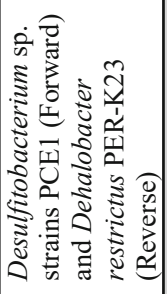 & 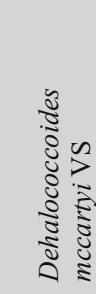 & 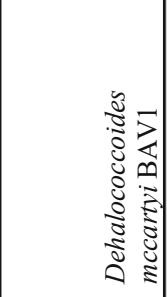 & 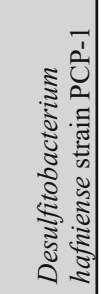 & 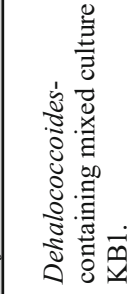 & 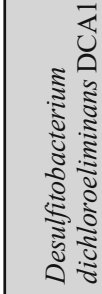 & 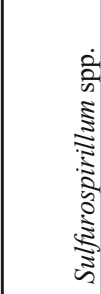 \\
\hline ปั) & $\underset{\Xi}{ }$ & $=$ & $=1$ & $=$ & పे & 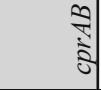 & $\begin{array}{l}\mathbb{Z} \\
\mathbb{Z}\end{array}$ & $\frac{\tilde{d}}{\tilde{d}}$ & $\underset{\$}{\mathbb{2}}$ \\
\hline
\end{tabular}




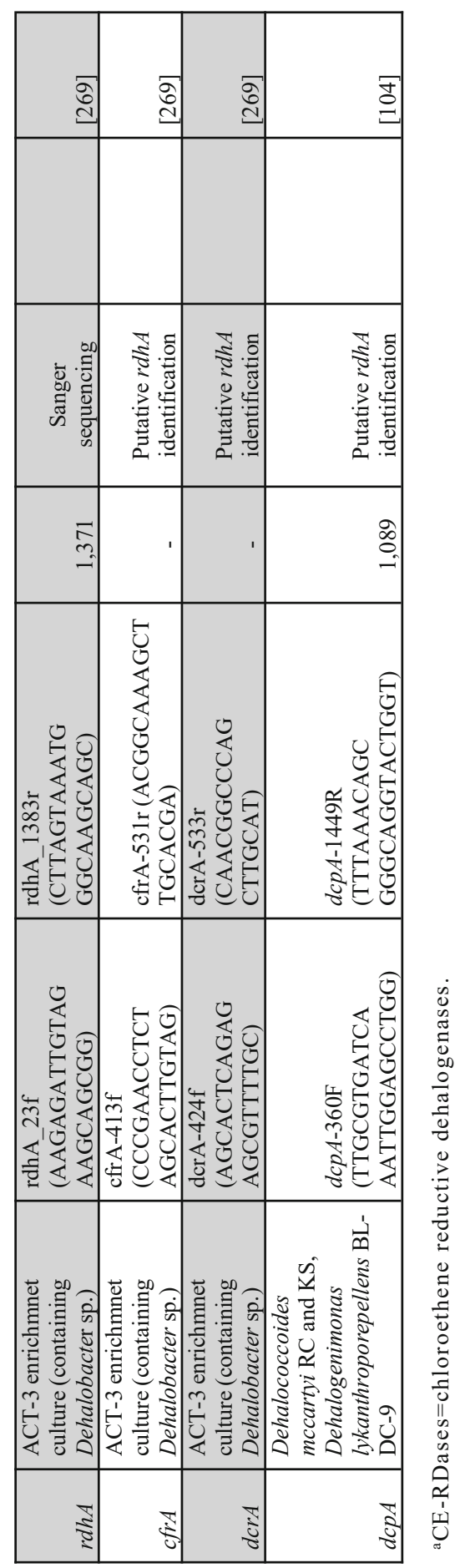




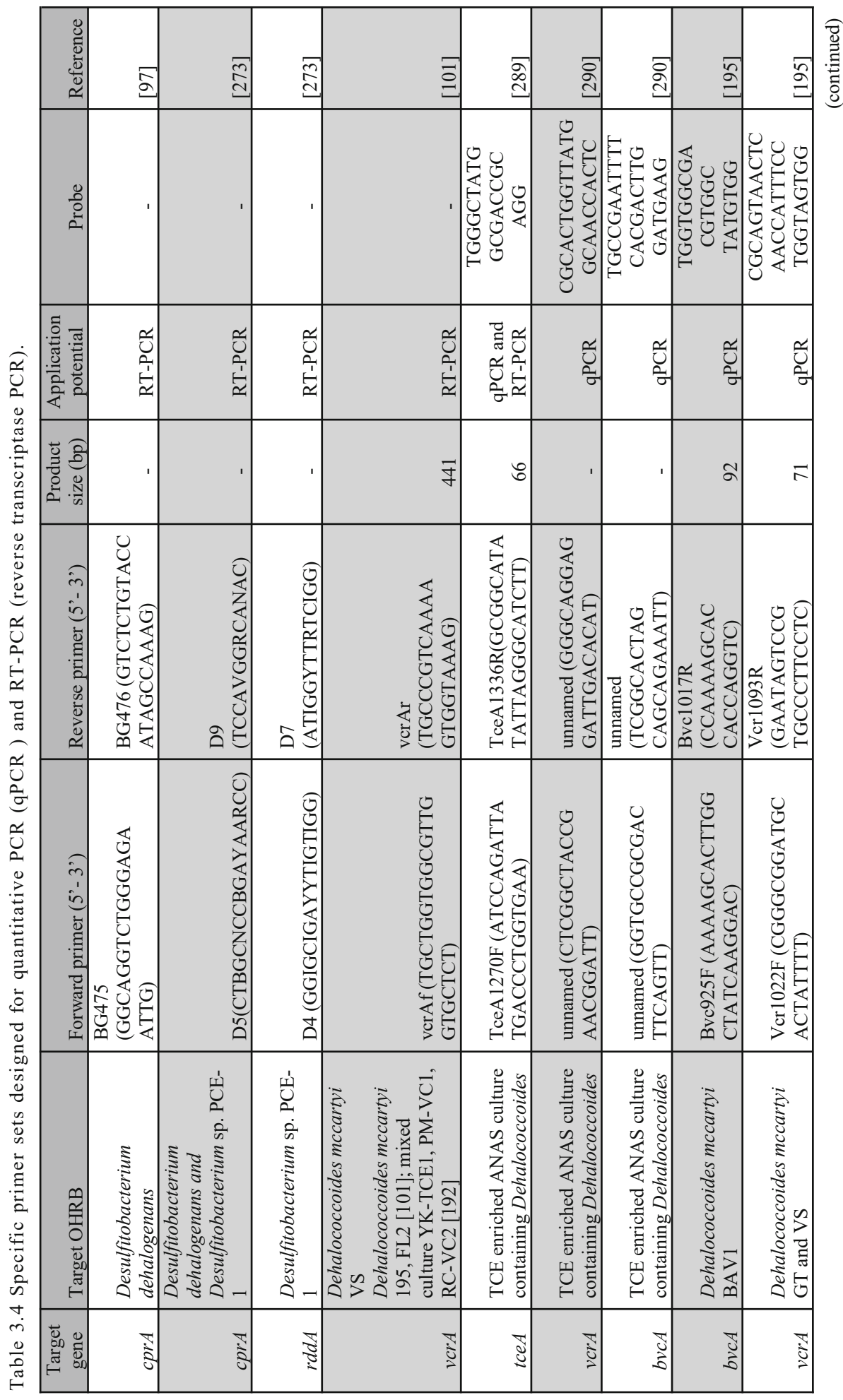




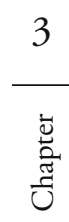

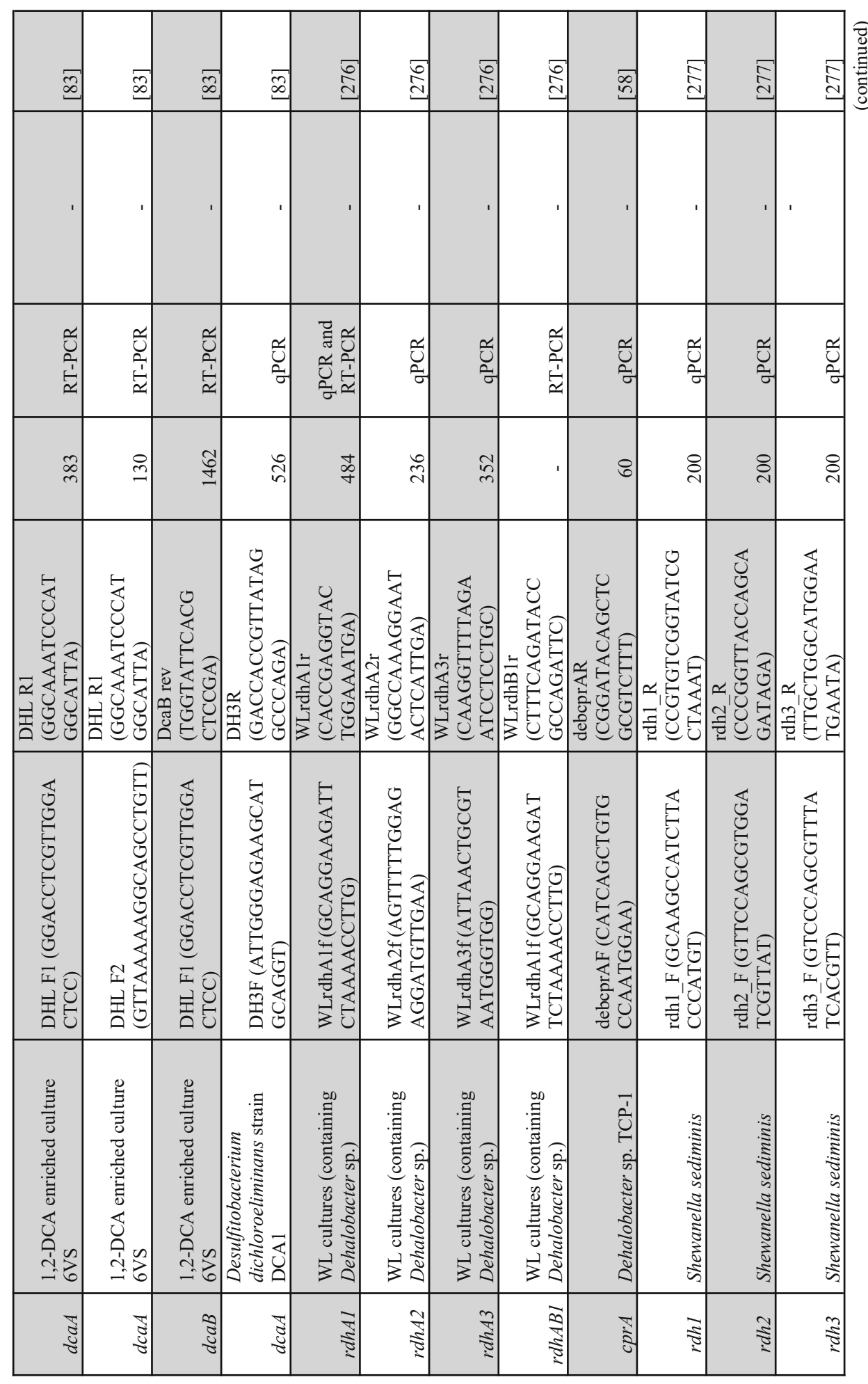




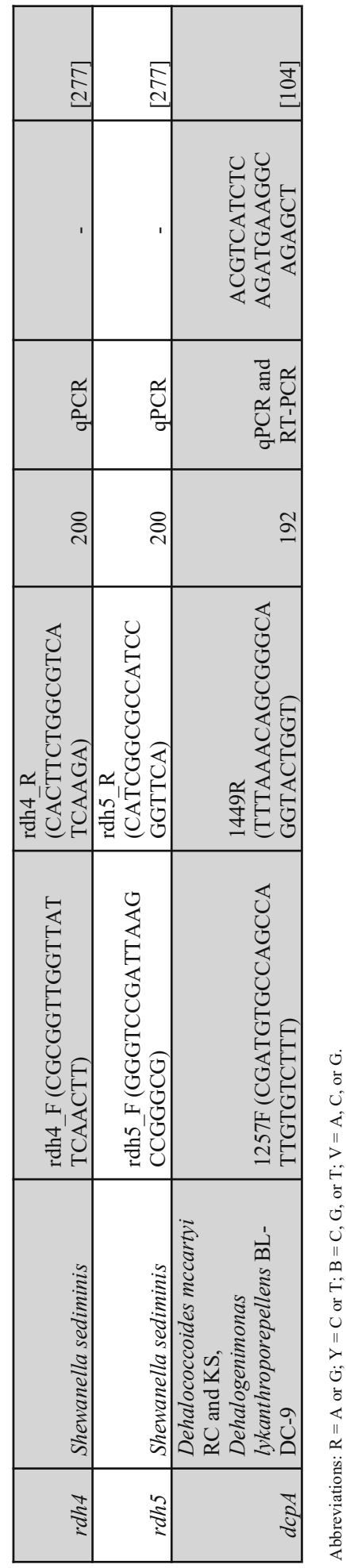


numbers to cell numbers, albeit with the restriction that actively growing cultures might contain multiple genomes per cell.

Primers have additionally been designed for reverse transcription, which, in conjunction with qPCR, was applied to quantify specific $r d h$ transcripts from microbial samples. From this, genes encoding the $\mathrm{A}$ and $\mathrm{B}$ subunits of reductive dehalogenases were found to be cotranscribed [83, 276, 291], confirming previous Northern blot hybridization and genespanning RT-PCR examinations of Desulfitobacterium dehalogenans transcripts [97]. Nevertheless, the accuracy of RT-PCR is limited by inefficiencies in reverse transcription and loss during sample processing. To account for this, Johnson [289] introduced an exogenous internal reference mRNA for normalization of RT-PCR, thereby improving the accuracy of quantification and allowing quantification of mRNA loss during specific steps, including RNA extraction, RT-PCR and qPCR.

\section{Conclusions}

Degenerate primers have been valuable tools for discovery of new $r d h A$ sequences. Their application is not costly and does not need complicated bioinformatics data analysis. Specific primers, on the other hand, have been successfully applied for quantitative analysis of OHRB and their biomarkers and functionality proofs of the identified genes. The hunt for new $r d h A s$ was moved to a high-throughput approach by combined application of primer-dependent analysis and next-generation amplicon sequencing [285]. Additionally, primer-independent metagenomic surveys are expected to further broaden the diversity of currently known $r d h$ genes, as has already been demonstrated in marine subsurface sediments [216]. Combined application of amplicon-based or metagenomic surveys with high-throughput quantitative analysis methods [288] should be instrumental in obtaining a comprehensive understanding of OHRB and their catabolic reductive dehalogenation gene pools.

\section{Acknowledgements}

The authors thank the BE-Basic Foundation (project MicroControl (8.004.01)) for financial support. Yue Lu was sponsored by a CSC Fellowship. 


\title{
Functional genomics of corrinoid
}

\author{
starvation in the organohalide-
}

\section{respiring bacterium Debalobacter}

\section{restrictus strain PER- K23}

This chapter has been published: Aamani Rupakula\#, Yue Lu\#, Thomas Kruse, Sjef Boeren, Christof Holliger, Hauke Smidt, Julien Maillard. 2015. Functional genomics of corrinoid starvation in the organohalide- respiring bacterium Dehalobacter restrictus strain PER-K23. Frontiers in Microbiology 5:751 \#equal contribution

Supplementary materials can be found online at: http://www.frontiersin.org/journal/10.3389/fmicb.2014.00751/abstract 


\begin{abstract}
De novo corrinoid biosynthesis represents one of the most complicated metabolic pathways in nature. Organohalide-respiring bacteria (OHRB) have developed different strategies to deal with their need of corrinoid, as it is an essential cofactor of reductive dehalogenases, the key enzymes in OHR metabolism. In contrast to Dehalococcoides mccartyi, the genome of Dehalobacter restrictus strain PER-K23 contains a complete set of corrinoid biosynthetic genes, of which $c b i H$ appears to be truncated and therefore non-functional, explaining the corrinoid auxotrophy of this obligate OHRB. Comparative genomics within Dehalobacter spp. revealed that one (operon-2) of the five distinct corrinoid biosynthesis associated operons present in the genome of $D$. restrictus appeared to be present only in that particular strain, which encodes multiple members of corrinoid transporters and salvaging enzymes. Operon-2 was highly up-regulated upon corrinoid starvation both at the transcriptional (346-fold) and proteomic level (46-fold on average), in line with the presence of an upstream cobalamin riboswitch. Together, these data highlight the importance of this operon in corrinoid homeostasis in D. restrictus and the augmented salvaging strategy this bacterium adopted to cope with the need for this essential cofactor.
\end{abstract}




\section{Introduction}

Corrinoids are essential cofactors for a wide variety of enzymes that facilitate reactions including rearrangements, methyl group transfers, and reductive dehalogenation [292]. A recent bioinformatic study has revealed that while $76 \%$ of 540 sequenced bacterial genomes contain corrinoid-dependent enzymes, only 39\% of these genomes encode the complete corrinoid biosynthesis pathway, suggesting that the salvage of corrinoids from the environment is an important process for many bacteria [293]. Both aerobic and anaerobic corrinoid biosynthesis pathways have been described showing few but significant differences, notably in tetrapyrrole ring contraction and the step at which cobalt is inserted into the ring [294, 295]. This pathway is complex and consists of approximately 30 reactions (see [294] for a recent review).

Organohalide respiration (OHR) is an anaerobic bacterial respiration process of environmental interest, as many anthropogenic halogenated organic compounds can be used as terminal electron acceptors by organohalide-respiring bacteria (OHRB) [7]. OHRB are capable to remove the halogens and therefore contribute to bioremediation of environments polluted with these compounds [10]. The key enzyme in OHR is the reductive dehalogenase (RDase) [46], which strictly depends on corrinoid cofactors for the dehalogenation reaction. Although the reaction mechanism has not yet been fully understood, RDases represent a particular family of corrinoid enzymes as they catalyze electron transfer rather than methyl transfer. Moreover the absence of corrinoid binding motif in RDase sequences reflects the base-off/his-off conformation of the corrinoid in the enzyme [78, 296]. In recent years, corrinoid biosynthesis and salvaging in OHRB regained substantial interest in the scientific community as exemplified by the following studies: an unusual corrinoid cofactor (norpseudo- $\mathrm{B}_{12}$ ) has been identified in the tetrachloroethene (PCE) RDase of Sulfurospirillum multivorans [297]; the lack of exogenous corrinoid had an effect on the RDase activity of Desulfitobacterium hafniense when cultivated with an alternative electron acceptor [298]; many essential corrinoid biosynthetic genes have been found on a plasmid in Geobacter lovleyi [128]; the involvement of the bacterial community accompanying members of Dehalococcoides mccartyi for corrinoid supply has been highlighted [93, 95, 237, 299]; modifying the lower ligand of the corrinoid had a severe effect on the activity of the PCE RDase of S. multivorans [145]. 
Contrasting situations have been observed regarding the ability of OHRB to produce corrinoid cofactors de novo. Both genome analysis and physiological studies have shown that the obligate OHR D. mccartyi is strictly dependent on exogenous corrinoid supply and that 5,6dimethylbenzimidazole can serve as nucleotide loop in corrinoid cofactors [40, 94, 95, 299, 300]. On the contrary, the facultative OHRB S. multivorans strain K and D. hafniense strains encode the full corrinoid biosynthetic pathway in their genome and have been shown to grow without any supply of corrinoid in the medium [20, 74, 301, 302].

Dehalobacter restrictus strain PER-K23 is an obligate OHRB only able to grow by dechlorinating tetra- and trichloroethene (PCE and TCE, respectively). It was first isolated from Rhine river sediment and since then always cultivated in the presence of exogenous vitamin $\mathrm{B}_{12}$ (cyanocobalamin) [52]. The PCE RDase (PceA) of D. restrictus has been extensively studied and revealed a $60-\mathrm{kDa}$ enzyme containing a corrinoid cofactor and two $4 \mathrm{Fe}-4 \mathrm{~S}$ clusters with estimated redox potential of $-350 \mathrm{mV}\left(\mathrm{Co}^{1+/ 2+}\right)$ and $-480 \mathrm{mV}(4 \mathrm{Fe}-$ $4 \mathrm{~S}^{2+/ 1+}$ ), respectively, and a specific dechlorination activity of $250 \mathrm{nkat} / \mathrm{mg}$. Spectrophotometric analysis of the corrinoid extracted from $D$. restrictus PceA with cyanide has shown a spectrum resembling the one of cyanocobalamin [296], although this method does not allow identifying corrinoid unambiguously. Analysis of the newly published genome of $D$. restrictus revealed the presence of a complete set of corrinoid biosynthetic genes where one gene, $c b i H$, is truncated due to a 101-bp deletion, likely responsible for the corrinoid auxotrophy of $D$. restrictus $[67,261]$ (Figure 4.1).

This present study aims to explore in detail the effect of corrinoid starvation on $D$. restrictus with a combination of comparative genomics, as well as transcription and proteome analysis.

\section{Materials and methods}

\section{Bacteria, plasmids, and growth conditions}

Dehalobacter restrictus strain PER-K23 (DSM 9455) was cultivated as described earlier [52, 64, 67]. Anaerobic serum flasks of $500 \mathrm{~mL}$ were supplemented with hydrogen as electron donor, inoculated with $2 \%(\mathrm{v} / \mathrm{v})$ inoculum, and finally $1 \%(\mathrm{v} / \mathrm{v})$ of $2 \mathrm{M}$ PCE dissolved in hexadecane was added as electron acceptor. Batch cultures of $D$. restrictus were cultivated in $300 \mathrm{~mL}$ medium at $30^{\circ} \mathrm{C}$ under gentle agitation $(100 \mathrm{rpm})$, and chloride release was used as an indicator of growth. Chloride concentration was measured with a Chlor-o-counter (Flohr Instrument, Nieuwegein, The Netherlands) as described earlier [249]. Cultures for proteomic 
analysis were prepared in triplicate with high, mid and low concentration of cyanocobalamin corresponding to 250,50 and $10 \mu \mathrm{g} / \mathrm{L}$, respectively.

Escherichia coli DH5 $\alpha$ was cultivated on liquid or solid LB medium containing $100 \mu \mathrm{g} / \mathrm{L}$ ampicillin after transformation with derivatives of the pGEM-T Easy vector (Promega, Duebendorf, Switzerland).

\section{Sequence retrieval and genome analysis}

All sequences mentioned in this study were taken from the recently published genome of $D$. restrictus strain PER-K23 [261] and from other Dehalobacter spp. genomes including Dehalobacter sp. E1 [66], Dehalobacter sp. DCA and sp. CF [303], Dehalobacter sp. FTH1 (RefSeq PRJNA199134, JGI genome project), Dehalobacter sp. UNSWDHB [304]. The original annotation of $D$. restrictus gene loci obtained in collaboration with the Joint Genome Institute (JGI project \#402027) was used here as the present study is the follow-up study of two previous reports where the JGI annotation was used $[67,261]$. Another version of $D$. restrictus genome was recently annotated by the automatic pipeline of the NCBI database and is available under accession number CP007033. Corresponding loci from both databases are given for the selected corrinoid proteome in Table S4.1.

The annotation of selected genes was verified using a manual search with BLAST [305]. Protein sequences were aligned using ClustalX v.2.0 [306]. Sequence maximum likelihood tree analysis was done with MEGA5 [307]. Cobalamin riboswitches (Cbl-RS) were identified using Rfam [308] and initially aligned using ClustalX and then corrected manually as described earlier [301]. Comparative genome analysis was performed using the Artemis Comparison Tool [309].

\section{Transcription analysis}

RNA was extracted using the TRIzol method according to [310] with the following modification. The DNaseI treatment was stopped by adding the DNase stop solution and incubating for $10 \mathrm{~min}$ at $65^{\circ} \mathrm{C}$. RNA concentration was estimated using the Nanodrop ND1000 spectrophotometer (Thermo Scientific, Ecublens, Switzerland). Reverse transcription was performed as described before [67].

Primers targeting each gene present immediately downstream of the five Cbl-RS of $D$. restrictus were designed. PCRs and cloning using the pGEM-T Easy vector, cloning, clone selection, sequencing and quantitative PCR were performed as described earlier [67]. Primer 
sequences, amplicon sizes, plasmids and quantitative PCR specifications are given in Table S4.2.

\section{Protein extraction and SDS-PAGE}

Cells were harvested by 10 min centrifugation at $12000 \times g$, washed twice with $25 \mathrm{~mL} 20 \mathrm{mM}$ Tris- $\mathrm{HCl}$ (pH 7.5), and then flash-frozen in liquid nitrogen. All biomass samples were stored at $-80^{\circ} \mathrm{C}$ until use. Cell pellets were resuspended in $0.5 \mathrm{~mL}$ lysis buffer $(100 \mathrm{mM}$ Tris/ $\mathrm{HCl}$, $\mathrm{pH} 7.5,4 \%$ sodium dodecyl sulfate, and $0.1 \mathrm{M}$ dithiothreitol) and then transferred to 2-mL protein LoBind tubes (Eppendorf, Hamburg, Germany). Protein extraction was done as described earlier [67]. Protein concentration was determined with the Qubit@ protein assay kit (Invitrogen, Eugene, OR, USA) following the manufacturer's instructions. Protein samples were stored at $-20^{\circ} \mathrm{C}$ until use. SDS-PAGE was done following standard procedures [311]. In brief, $15 \mu \mathrm{g}$ of proteins from each sample were loaded in separate lanes in gels containing $10 \%$ SDS. Gels were stained with Coomassie brilliant blue R250 (Merck, Darmstadt, Germany) and scanned using a GS-800 calibrated densitometer (Bio-Rad, Hercules, CA, USA). The Quantity One basic software package was used to quantify the intensity of lanes. Series of gels were prepared and analyzed until less than $5 \%$ differences in the intensity between any lanes were achieved.

\section{Gel digestion and peptides purification}

In-gel digestion of proteins and purification of peptides were done following a modified version of the protocol described earlier [67]. Disulphide bridges in proteins were reduced by covering whole gels with reducing solution $(10 \mathrm{mM}$ dithiothreitol, $\mathrm{pH} 7.6$, in $50 \mathrm{mM}$ $\mathrm{NH}_{4} \mathrm{HCO}_{3}$ ), and the gels were incubated at $60^{\circ} \mathrm{C}$ for $1 \mathrm{~h}$. Alkylation was performed for $1 \mathrm{~h}$ by adding $25 \mathrm{~mL}$ of iodoacetamide solution $(10 \mathrm{mM}$ iodoacetamide in $100 \mathrm{mM}$ Tris- $\mathrm{HCl}, \mathrm{pH}$ 8.0). Gels were thoroughly rinsed with $\mathrm{dd}_{2} \mathrm{O}$ water in between steps. Each lane of SDSPAGE gels was cut into three equally sized slices, and each slice was cut into approximately 1 $\mathrm{mm}^{3}$ cubes and transferred to separate $0.5 \mathrm{~mL}$ protein LoBind tubes (Eppendorf, Hamburg, Germany). Enzymatic digestion was done by adding $50 \mu \mathrm{L}$ of trypsin solution $(5 \mathrm{ng} / \mu \mathrm{L}$ trypsin in $50 \mathrm{mM} \mathrm{NH}_{4} \mathrm{HCO}_{3}$ ) to each tube, and incubating at room temperature overnight with gentle shaking. Extraction of peptides was performed with manual sonication in an ultrasonic water bath for $1 \mathrm{~s}$ before the supernatant was transferred to a clean protein LoBind tube. Additional peptides were recovered by adding $25 \mu \mathrm{L}$ of $2.5 \%(\mathrm{v} / \mathrm{v})$ trifluoroacetic acid to the gel pieces, which were sonicated for $2 \mathrm{~s}$ before the supernatant was combined with the first supernatant obtained. Peptides were purified with a C18 Empore disk as previously described 
[312]. Acetonitrile in the samples was removed by using a concentrator vacuum centrifuge. Finally, sample volume was adjusted to $50 \mu \mathrm{L}$ with $0.1 \%(\mathrm{v} / \mathrm{v})$ formic acid.

\section{nLC-MS/MS and data analysis}

Peptides derived from extracted and digested proteins were analyzed by nLC-MS/MS (Biqualys, Wageningen, The Netherlands) as described earlier [313]. MaxQuant v.1.3.0.5 [314] with default settings for the Andromeda search engine [314] in the label free quantitation mode was used to analyze MS and MS/MS spectra, except that extra variable modifications were set as described before [67]. A protein database of D. restrictus was generated from the whole genome sequence [261] using the Artemis genome browser (release 15.0.0). Also, a contaminant database including sequences of common contaminants like trypsin, BSA and human keratins $[67,315]$ was used. Further filtering and bioinformatics analysis was performed with Perseus software v. 1.3.0.4 as described before [316]. Also, protein groups with a logarithmic label-free quantitation (LFQ) intensity of zero for all treatments were deleted from the MaxQuant result table. Subsequently, remaining Log LFQ zero values were replaced by 5 (slightly below the lowest value measured) in order to make sensible ratio calculations possible. Students T-test was used to identify significant differences in the proteome when comparing logarithmic LFQ values obtained from two culture conditions.

\section{Results}

Corrinoids are essential as a growth factor for D. restrictus [52]. The corrinoid present in the PCE reductive dehalogenase (PceA) of $D$. restrictus is presumably similar to the type added to the medium, i.e. cobalamin [52]. Detailed analysis of the genome of D. restrictus revealed a seemingly complete corrinoid biosynthesis pathway. Compared to other Dehalobacter genomes, however, a 101-bp fragment was found to be missing in the $c b i H$ gene of $D$. restrictus $[67,261]$ (Figure S4.1). The present study aimed specifically at obtaining a better understanding of the corrinoid metabolism in D. restrictus.

\section{Growth of $D$. restrictus under corrinoid-limiting conditions}

The full corrinoid biosynthetic pathway was described earlier [67]. A modified and extended version of it is depicted in Figure 4.1. Briefly, the pathway can be divided in two branches, namely the upper corrinoid biosynthesis (UCB) and the nucleotide loop assembly (NLA), which are connected at the level of ado-cobyric acid. 

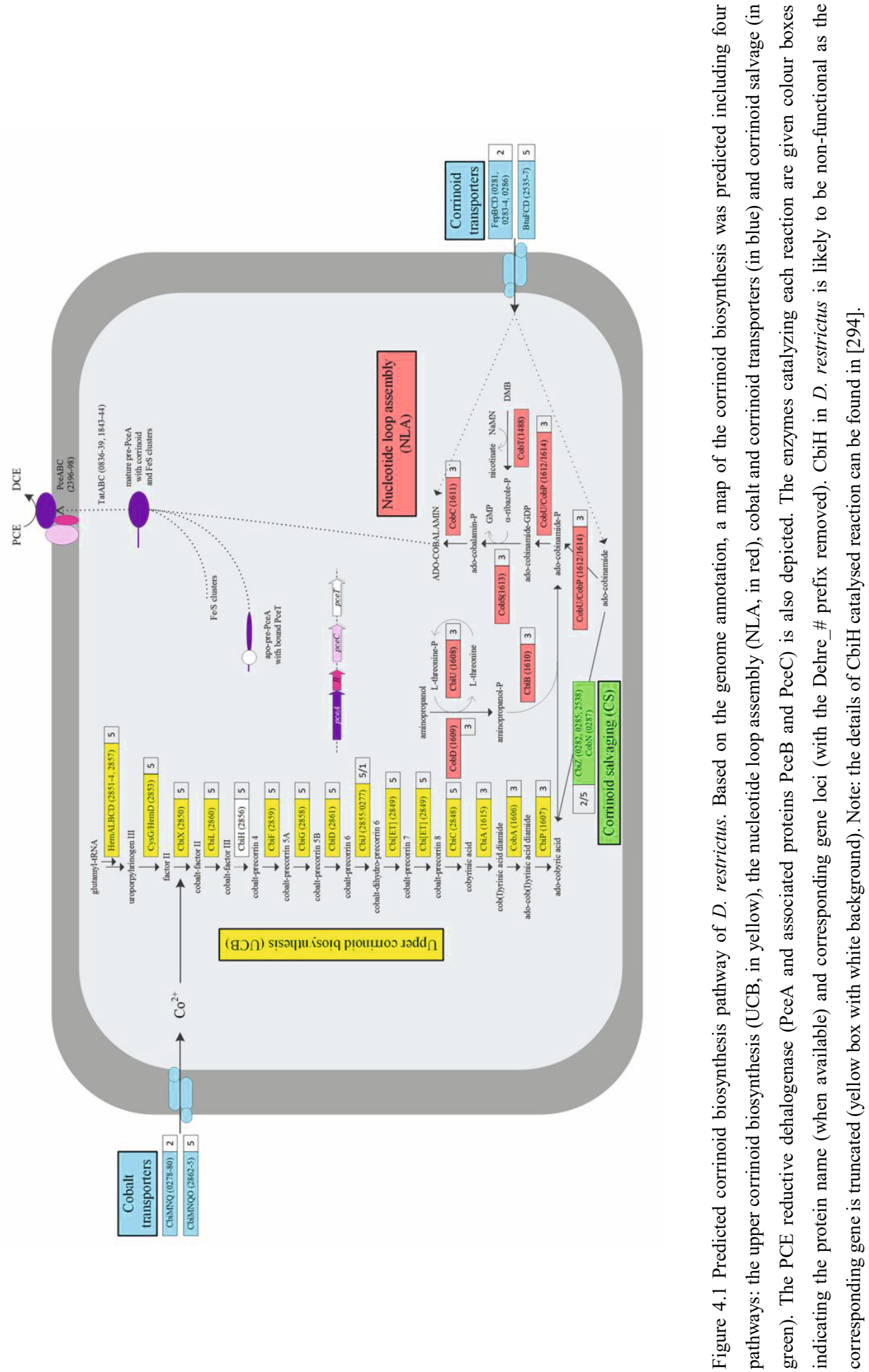
In the present study, batch cultures were cultivated with addition of $250 \mu \mathrm{g} / \mathrm{L}$ cyanocobalamin to the growth media. An experiment was performed to assess the effects of lowering the initial corrinoid concentration in the medium $(250,50,10,1 \mu \mathrm{g} / \mathrm{L}$ and no corrinoid) on dechlorination, which for this obligate OHRB is also a good estimation for growth (Figure 4.2) [52]. The extent of PCE dechlorination was the same in cultures provided with 50 or $250 \mu \mathrm{g} / \mathrm{L}$ corrinoid demonstrating that the former was enough to reach the maximum dechlorination capacity. In contrast, the chloride release was only half of the maximum in cultures supplemented with $10 \mu \mathrm{g} / \mathrm{L}$ corrinoid, implying that availability of corrinoids was a limiting factor. Further lowering the corrinoid concentration to 1 or $0 \mu \mathrm{g} / \mathrm{L}$ resulted in negligible levels of dechlorination, and therefore growth was assumed to be abolished in these cultures.

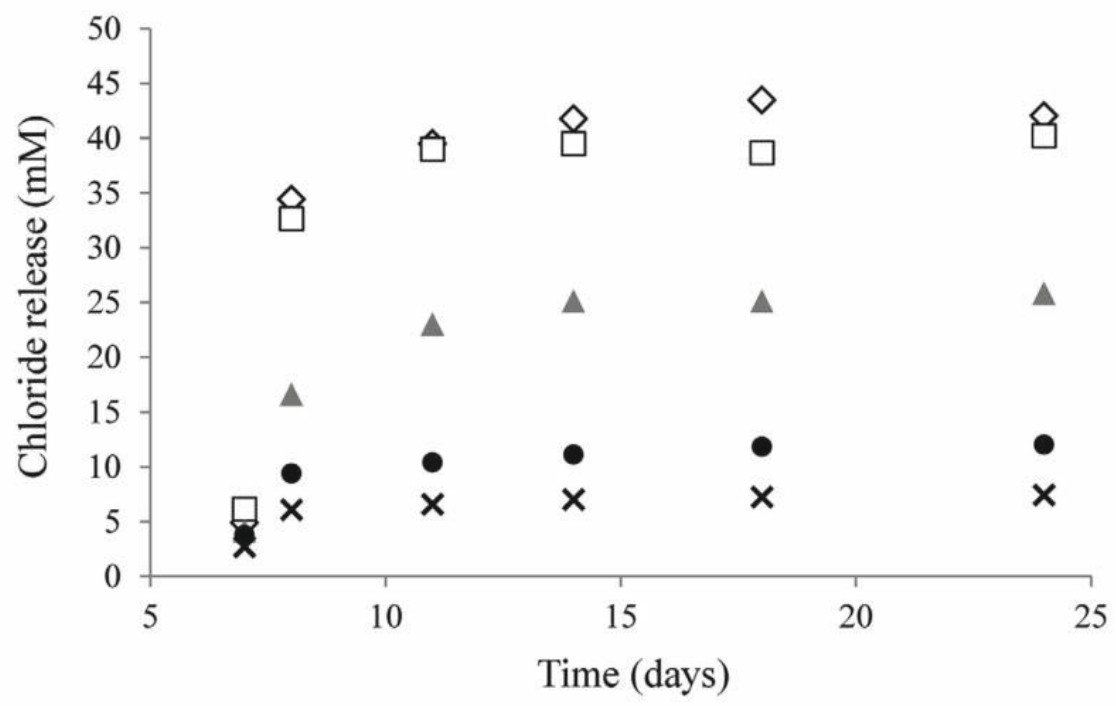

Figure 4.2 Corrinoid starvation effect on PCE dechlorination by D. restrictus by lowering the initial concentration of corrinoid supplemented into the medium. Corrinoid concentration: $250 \mu \mathrm{g} / \mathrm{L}$ (white diamonds), $50 \mu \mathrm{g} / \mathrm{L}$ (white squares), $10 \mu \mathrm{g} / \mathrm{L}$ (grey triangles), $5 \mu \mathrm{g} / \mathrm{L}$ (black circles) and $1 \mu \mathrm{g} / \mathrm{L}$ (black crosses).

\section{Corrinoid metabolic gene arrangement in $D$. restrictus}

The complete corrinoid biosynthetic and uptake pathway is genetically encoded in $D$. restrictus. These genes can be divided into four functional groups depending on the part of the pathway they encode for (Figure 4.1). The first group denoted as the upper corrinoid 
biosynthesis (UCB) pathway genes contains genes required to synthesize ado-cobyric acid. The second group consists of genes required for synthesis and the nucleotide loop assembly (NLA) of corrinoids, and the third functional group comprises the corrinoid salvaging (CS) pathway, i.e. genes involved in remodeling corrinoid intermediates salvaged from the environment into ado-cobyric acid (cbiZ gene family). The fourth group harbors both cobalt and corrinoid transporter encoding genes (CT).

Most genes associated with corrinoid metabolism are arranged in the genome of $D$. restrictus in five gene clusters (referred to as operon-1 to -5 ), which are roughly organized according to the function they play in corrinoid biosynthesis (Figure 4.3). Most proteins involved in the UCB pathway are encoded in operon-5 with the exception of the three last steps that are catalyzed by the product of genes present in operon-3. This latter operon also codes for the enzymes involved in the NLA pathway. Within operon-3, the locus Dehre_1608 was initially annotated as a phosphoglycerate mutase, but shows also sequence similarity with archaealtype homoserine kinase (with conserved domain TIGR02535) involved in the synthesis of threonine. Here, we propose it could act as an L-threonine kinase (in analogy to PduX in Salmonella [317]), which might therefore be involved in the production of aminopropanolphosphate. No $p d u X$ homolog could be identified in D. restrictus, suggesting that this function is fulfilled by the gene product of Dehre_1608. Hence, we propose to name it $c b i U$. Operon-1 contains a homolog of $c b i J$ (besides the cbiJ/cysG gene, Dehre_2855, present in the conserved biosynthesis operon-5), and a set of genes coding for the energy-coupling factor-type CbiMNQ cobalt transporter. An additional, albeit different cbiMNQO gene cluster is also present at the 5'-end of operon-5 together with the genes for the UCB pathway. Operon-2 harbors a combination of genes coding for transporters (with sequence similarity to FepBCD/BtuCDE ABC-type transporters) likely involved in corrinoid transport, the genes for two different salvaging enzyme (CbiZ) paralogues (Dehre_0282 and_0285), a gene cluster encoding the cobaltochelatase CobN (Dehre_0287), and several subunits of a magnesium chelatase complex. All proteins encoded in operon-2 share between 50 and $77 \%$ sequence identity with homologous proteins present in the non-dechlorinating Firmicute Acetobacterium woodii. Finally, operon-4 contains a gene cluster coding for an ABC-type corrinoid transporter (BtuFCD) and another copy of cbiZ (Dehre_2538). Two additional genes potentially involved in corrinoid biosynthesis (cobT/Dehre_1488 and cobB/cobQ, Dehre_2360) are located elsewhere in the genome and not in one of the five operons. 

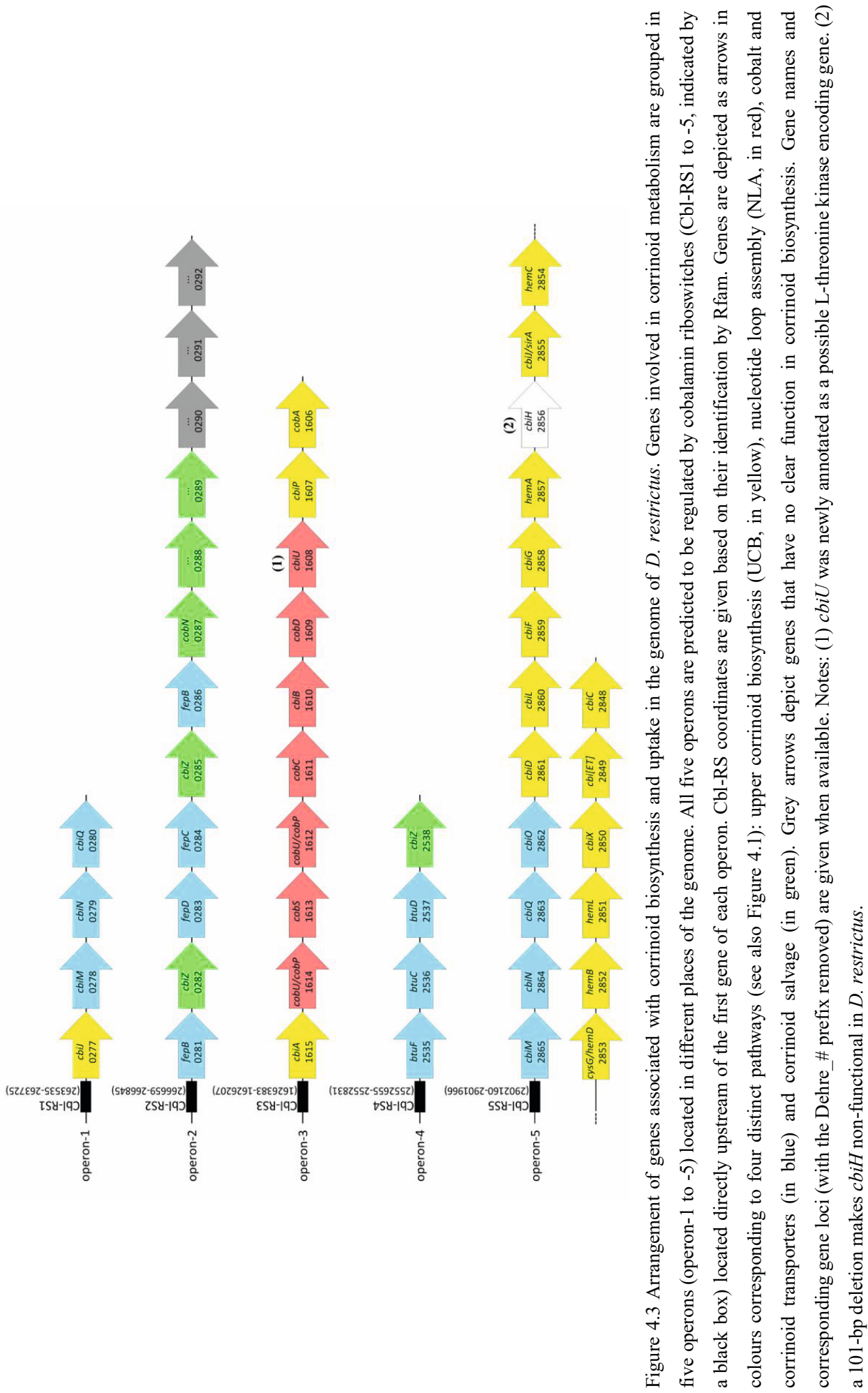


\section{Comparative genomics of corrinoid operons in Dehalobacter spp.}

The genome of $D$. restrictus was compared with newly available genomes of Dehalobacter spp. strains DCA, CF, E1, FTH1, and UNSWDHB with regard to the organization of corrinoid operons (Table 4.1 and Table S4.1). Synteny maps for operon-1 and -2 (Figure S4.2), and for operon-3, -4 and -5 (Figure S4.3 to S4.5, respectively) are given as Supplementary materials. Operon-1 is conserved in D. restrictus and Dehalobacter sp. E1 but absent in all other genomes. Operon-2, which is directly following operon-1 in D. restrictus, is lacking in all other Dehalobacter spp. for which genome sequences are available to date. However, a detailed analysis of Dehalobacter sp. E1 suggests that operon-2 was lost in that strain as the sequence conservation with $D$. restrictus is extended slightly beyond operon-1 but is readily interrupted within the homolog of Dehre_0281 (the first gene of operon-2 in $D$. restrictus). This deletion in strain E1 includes all of the remaining genes operon- 2 and beyond, as a 5'-truncated version of Dehre_0297 is again found in strain E1 (Figure S4.2, panel C). D.restrictus operon-3 to -5 are fully conserved in all Dehalobacter spp. with the exception of another deletion in the proximal region of operon-5 in strain E1 (Figure S4.5).

Table 4.1 Comparative genomics of corrinoid operons in Dehalobacter spp.

\begin{tabular}{|l|c|c|c|c|c|c|}
\hline Operon & $\begin{array}{c}\text { Dehalobacter } \\
\text { restrictus }\end{array}$ & $\begin{array}{c}\text { Dehalobacter } \\
\text { sp. E1 }\end{array}$ & $\begin{array}{c}\text { Dehalobacter } \\
\text { sp. DCA }\end{array}$ & $\begin{array}{c}\text { Dehalobacter } \\
\text { sp. CF }\end{array}$ & $\begin{array}{c}\text { Dehalobacter } \\
\text { sp. FTH1 }\end{array}$ & $\begin{array}{c}\text { Dehalobacter } \\
\text { sp.UNSWDH } \\
\text { B }\end{array}$ \\
\hline 1 & + & + & - & - & - & - \\
\hline 2 & + & - & - & - & - & - \\
\hline 3 & + & + & + & + & + & + \\
\hline 4 & + & + & + & + & + & + \\
\hline 5 & + & partial & + & + & + & + \\
\hline cbiH & deletion & intact & intact & intact & intact & intact \\
\hline
\end{tabular}

In Desulfitobacterium hafniense in contrast, the corrinoid biosynthesis genes are organized in two operons. The major operon (corresponding to DSY4057-4072 in D. hafniense strain Y51) encodes proteins of the UCB pathway and part of the NLA pathway, while a 3-gene operon (DSY2114-2116) encodes for the remaining NLA proteins [74, 301]. Some proteins encoded by $D$. restrictus operon-2 have their counterpart in other OHRB. For example, corrinoid transporters are present in most OHRB, however, corrinoid producers such as Desulfitobacterium hafniense and Sulfurospirillum multivorans do not harbor any cbiZ homologous gene. In Dehalococcoides mccartyi in contrast, multiple cbiZ genes are present in the genomes (Figure S4.6). 
All proteins encoded in operon- 2 of $D$. restrictus share between 50 and $77 \%$ sequence identity with homologous proteins present in the non-dechlorinating Firmicute Acetobacterium woodii. A high level of genetic synteny was further identified between operon-2 of D. restrictus and a part of the genome of $A$. woodii (GenBank NC_016894.1, [318]) (Figure S4.7). Significant sequence similarity of individual proteins of operon-2 was mostly found with homologs of some other members of Chlostridia and a few $\delta$-Proteobacteria (data not shown).

\section{Identification of cobalamin riboswitches in D. restrictus}

Upstream of each of the five corrinoid biosynthesis-related operons in D. restrictus a distinct cobalamin riboswitch (Cbl-RS) was identified using Rfam. These five sequences were manually refined in a similar way as done previously for the Cbl-RS sequences of Desulfitobacterium hafniense [301]. The alignment of structurally conserved regions of $D$. restrictus riboswitches (Cbl-RS01 to -RS05) was compared to E. coli btuB Cbl-RS (Figure S4.7). In contrast to E. coli Cbl-RS, which is regulated at the level of translation [319], all five D. restrictus Cbl-RS sequences end with a predicted transcriptional terminator, suggesting that the regulation operates at the level of transcription.

\section{Transcriptional analysis of corrinoid biosynthesis operons in $D$. restrictus}

The transcription of genes located directly downstream of the Cbl-RS in D. restrictus was analyzed for cells cultivated in the presence of high $(250 \mu \mathrm{g} / \mathrm{L})$ and low $(10 \mu \mathrm{g} / \mathrm{L})$ corrinoid concentration, and after corrinoid replenishment from low to high concentrations (Figure 4.4). Quantitative PCR was applied on complementary DNA targeting the first gene located directly downstream of each cobalamin riboswitch. Analysis of corrinoid-starved D. restrictus RNA revealed a higher transcription level of these genes, confirming an active regulation of the respective riboswitches at transcriptional level. Two hours after corrinoid replenishment, transcription of all selected genes was again repressed to the same level as observed under high corrinoid concentration. However, individual responses were significantly different. Indeed, the most pronounced effect was observed for two genes, namely Dehre_0277 (73-fold repression) and _0281 (346-fold), corresponding to the first genes in operon-1 and -2 in $D$. restrictus, respectively.

\section{Proteome analysis of corrinoid starvation in $D$. restrictus}

Comparative whole-proteome analysis was done on D. restrictus PER-K23 cells cultivated in the presence of 250 (high), 50 (mid) or 10 (low) $\mu \mathrm{g} / \mathrm{L}$ cyanocobalamin. A total of 1195 proteins were detected, corresponding to $42 \%$ of the predicted 2826 proteins encoded on the 


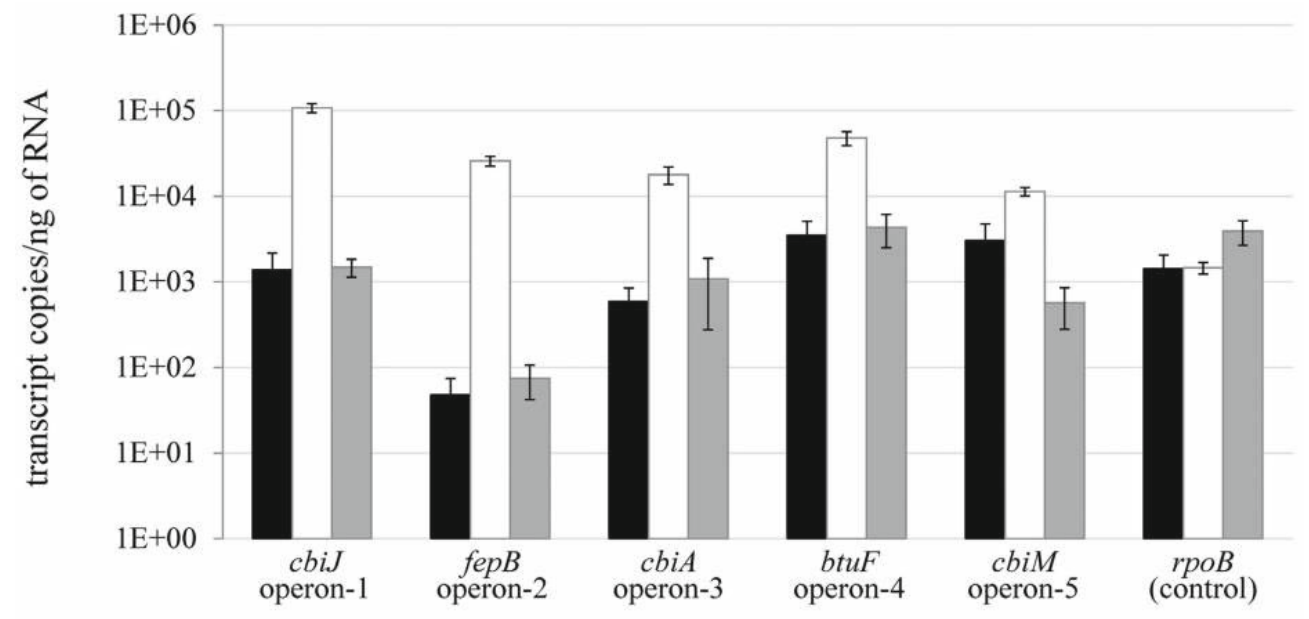

Figure 4.4 Transcriptional analysis of cobalamin riboswitch-dependent genes in D. restrictus. The transcription of the genes located directly downstream of the cobalamin riboswitches in operon-1 to -5 was analyzed. The housekeeping gene $r p o B$ was used as unregulated control. The black bars indicate the transcriptional level under corrinoid standard conditions $(250 \mu \mathrm{g} / \mathrm{L})$, the white bars under corrinoid starvation conditions $(10 \mu \mathrm{g} / \mathrm{L})$, and the grey bars show the transcriptional level $2 \mathrm{~h}$ after replenishment of the latter cultures with $250 \mu \mathrm{g} / \mathrm{L}$ cyanocobalamin. The data show the mean of triplicate cultures with standard deviation.

genome [261]. The majority of the detected proteins (1175) were identified in cells from all the tested cyanocobalamin concentrations (Table S4.4). Normalized LFQ protein intensities were used to compare relative abundances of proteins between different cyanocobalamin treatments. A minimal change of 3-fold in LFQ protein intensity was considered throughout the study. The abundance of 44 proteins showed significant difference $(\mathrm{P}<0.01)$ between high $(250 \mu \mathrm{g} / \mathrm{L})$ and low $(10 \mu \mathrm{g} / \mathrm{L})$ corrinoid concentration, and the relative abundance of another 29 proteins showed more than 10 -fold changes, albeit not significant due to high variation between triplicates (Figure S4.8). The results for protein abundance ratios between high and mid, and between mid and low are in the same range (see Table S4.3).

Proteins associated with cobalamin biosynthesis were further analyzed. A complete de novo corrinoid biosynthesis pathway was predicted in the genome of $D$. restrictus starting from glutamyl-tRNA to cobalamin (Figure 4.1) [67, 261]. All proteins required for biosynthesis of ado-cobyric acid from cobalt-precorrin 5B were identified in proteomic data including $\mathrm{CbiD}$ (cobalamin biosynthesis protein, Dehre_2861), an alternative CbiJ (precorrin-6x reductase, Dehre_0277) and Cbi[ET] (precorrin-6Y methyltransferase, Dehre_2849), which were not dectected in a previously analyzed proteome from D. restrictus [67]. However, CbiH 
(precorrin-3B C17-methyltransferase, Dehre_2856), CbiG (cobalamin biosynthesis protein, Dehre_2858) and CbiJ/CysG (precorrin-6x reductase, Dehre_2855) belonging to the UCB pathway and CobS (cobalamin 5'-phosphate synthase, Dehre_1613) of the NLA pathway were not found in the current proteome analysis. The lack of $\mathrm{CbiH}$ in the proteome is in line with the observation of a 101-bp deletion in $c b i H$ likely leading to a non-functional gene (Figure S4.1), thus likely to explain why D. restrictus requires exogenous corrinoids supply to the growth medium.
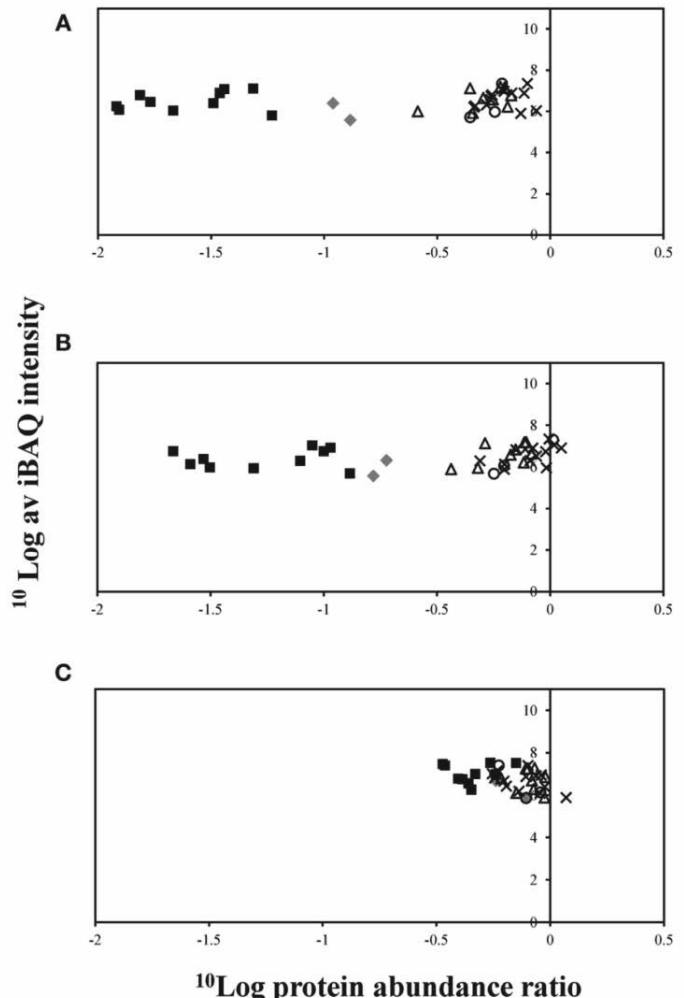

Figure 4.5 Proteins associated with corrinoid biosynthesis and uptake, extracted from D. restrictus cells cultivated in the presence of different cyanocobalamin concentrations. (A) Proteomics analysis of cells cultivated in the presence of high $(250 \mu \mathrm{g} / \mathrm{L}) v s$. low $(10 \mu \mathrm{g} / \mathrm{L})$ corrinoid concentrations; (B) high $(250 \mu \mathrm{g} / \mathrm{L}) v s$. mid $(50$ $\mu \mathrm{g} / \mathrm{L})$ corrinoid concentrations; (C) mid (50 $\mu \mathrm{g} / \mathrm{L}) v s$. low $(10 \mu \mathrm{g} / \mathrm{L})$ corrinoid concentrations. Proteins encoded by corrinoid operon-1 (grey diamonds), operon-2 (black squares), operon-3 (white triangles), operon-4 (white circles) and operon-5 (black crosses) are shown. Logarithmic average of iBAQ (Intensity based absolute quantitation) value is plotted against the $\log$ value of protein abundance ratio based on LFQ value. CbiM (Dehre_0278) was left out because it could not be identified in two replicates of cultures under low corrinoid concentration. 
Previously, the presence of one ABC-type cobalt transporter (Dehre_0850-0852) and two energy-coupling factor-type cobalt transporters (Dehre_0278-0280 and Dehre_2862-2865) was predicted in the genomic study of D. restrictus [67]. Here, we identified CbiM (Dehre_0278), CbiQ (Dehre_0280), CbiO (Dehre_2862) and CbiN (Dehre_2864) in the proteome dataset obtained in this study (Table S4.4).

Relative abundance of proteins associated with corrinoid biosynthesis and salvaging pathways was further analyzed. Interestingly, nearly all proteins associated with corrinoid biosynthesis and salvaging pathways were up-regulated under corrinoid limiting growth conditions (Figure 4.5 and Table S4.5). As expected the overall largest change in the abundance of proteins related to corrinoid biosynthesis and salvaging pathways was observed when comparing the proteome of cells cultivated at high vs. low concentration of cyanocobalamin (Figure 4.5A). The corrinoid metabolism differed more strongly when comparing cells cultivated in the presence of high $v s$. mid than mid $v s$. low concentrations (Figure $4.5 \mathrm{~B}$ and $5 \mathrm{C}$ ).

Proteins encoded by operon-2 showed the largest change in protein abundance ratios with on average 46-fold up-regulation when comparing cells cultivated at low vs. high corrinoid concentrations (Figure 4.5A). Operon-2 encodes proteins predicted to be involved in corrinoid salvaging or corrinoid transport. Among these are two predicted CbiZ proteins, amidohydrolases required for salvaging the corrinoid precursor cobinamide, which were upregulated 80-fold (Dehre_0285) and 58-fold (Dehre_0282) under corrinoid starvation, respectively (Table S4.4). Furthermore, proteins encoded in operon-1 including an energycoupling factor-type cobalt transporter (Dehre_0278-0280) which is likely to be involved in the cobalt uptake process, and a precorrin-6x reductase (Dehre_0277), were on average 8-fold up-regulated when comparing cells cultivated in the presence of low vs. high corrinoid concentrations. Fewer changes were found for proteins encoded by the three remaining corrinoid-related operons under the different corrinoid conditions.

\section{Discussion}

\section{Corrinoid biosynthesis of $D$. restrictus and other OHRB}

In the present study, $\geq 50 \mu \mathrm{g} / \mathrm{L}$ of cyanocobalamin was required for $D$. restrictus to reach its maximum PCE dechlorination, in line with previous observation that this organism depends on externally supplemented corrinoids [52]. The genome of $D$. restrictus encodes a complete set of corrinoid biosynthesis genes, with the exception of a non-functional $c b i H$ gene, suggesting that tetrapyrrole ring contraction does not occur here and represents a dead-end in 
the biosynthesis pathway. Comparative genomic analysis among other Dehalobacter spp. revealed that an intact $c b i H$ gene is present in all other genomes. However, little is known about the capacity of other members of this genus to de novo synthesize corrinoids. Indeed they only have been studied under growth conditions with external addition of cyanocobalamin or in co-cultures $[55,59,61,66,304]$. Similarly, strains of Dehalococcoides mccartyi, which are also obligate OHRB, are corrinoid-auxotroph [40]. Unlike D. restrictus, the corrinoid auxotrophy in D. mccartyi strains is due to the lack of the complete biosynthetic pathway. Instead they rely on uptake of extracellular corrinoids via the salvaging pathway and on remodelling of incomplete or non-functional corrinoids in the presence of appropriate free lower ligands, among which 5,6-dimethlybenzimidazole plays a key role [93, 94, 299, 300, 320]. Interestingly, most facultative OHRB such as Sulfurospirillum multivorans [297], Desulfitobacterium hafniense [74, 301] or Geobacter lovleyi [128] are capable of de novo biosynthesis of corrinoids.

The genome of D. restrictus encodes five well-organized operons containing most of the corrinoid biosynthesis-associated genes. Comparing the genomes of the sequenced Dehalobacter spp. revealed that D. restrictus harbours an extra set of genes (operon-2) coding for putative corrinoid transporters and salvaging enzymes (CbiZ and cobaltochelatases), suggesting an augmented capacity for corrinoid uptake and remodelling compared to other Dehalobacter spp. The importance of cbiZ genes in remodelling corrinoids has been already demonstrated for D. mccartyi [91, 92, 300]. The role of operon-2 in D. restrictus was evidenced by the significant up-regulation of the corresponding enzymes when corrinoid concentration in the medium was lowered. This result clearly showed that D. restrictus has developed a unique strategy to cope, at least partially, with its lack of corrinoid biosynthesis under unfavourable corrinoid conditions. The presence of additional cbiZ genes in operon-2 raises the questions of the functional redundancy vs. specificity of multiple CbiZ proteins within a single strain, and of the origin of the additional cbiZ genes present in D. restrictus. While in vitro biochemical investigations would be required to answer the first question, a detailed analysis of CbiZ sequence homology (Figure S4.6) revealed that the two additional CbiZ proteins in D. restrictus show a high level of sequence identity with CbiZ homologues present in Acetobacterium woodii, a corrinoid-producing bacterium [321], which has been well-characterized for the Wood-Ljungdahl pathway that also requires corrinoids as an essential cofactor [322]. The high degree of genomic synteny identified between the corrinoid operon-2 of $D$. restrictus and $A$. woodii suggests that $D$. restrictus, but not the other members 
of the Dehalobacter genus, most probably acquired operon-2 by horizontal gene transfer and successfully exploited this operon to partically alleviate the loss of a functional $c b i H$ gene.

\section{Effect of corrinoid starvation on $\boldsymbol{D}$. restrictus metabolism}

Reduction of cyanocobalamin amendment in the growth medium strongly inhibited PCE dechlorination by $D$. restrictus. It also had a profound effect on $D$. restrictus corrinoid metabolism both at the level of transcription and at the proteome level. While changing from high, $(250 \mu \mathrm{g} / \mathrm{L})$ corrinoid concentration to an intermediate concentration $(50 \mu \mathrm{g} / \mathrm{L}), D$. restrictus responded by up-regulating proteins associated with corrinoid transport and salvaging pathways encoded in operon- 1 and -2 , allowing the strain to reach the same PCE dechlorination level as observed during high corrinoid concentration. Decreasing the corrinoid concentration even further to $10 \mu \mathrm{g} / \mathrm{L}$ showed, however, that, while the extent of PCE dechlorination was strongly affected, the amount of corrinoid-associated proteins did not notably change when compared to cells cultivated in the presence of $50 \mu \mathrm{g} / \mathrm{L}$. This indicates that at corrinoid concentrations as low as $10 \mu \mathrm{g} / \mathrm{L}, D$. restrictus was not able to compensate the lack of externally provided corrinoids by increased corrinoid transport and salvaging.

The presence of cobalamin riboswitches directly upstream of the five corrinoid operons in $D$. restrictus already suggested an active repression at the level of transcription by cyanocobalamin. Similar to transcriptional studies on D. mccartyi [323] and D. hafniense [301], the cobalamin riboswitches of D. restrictus responded to addition of excess cyanocobalamin, and the level of repression of the gene located directly downstream of the riboswitches correlated well with the proteomic data, showing the strongest effect for cbiJ (Dehre_0277, operon-1) and for fepB (Dehre_0281, operon-2). The sequence of individual cobalamin riboswitches is likely responsible for their differential responsiveness towards cobalamin concentration, as both their affinity to cobalamin and the strength which the expression platform exerts on transcriptional repression are sequence dependent. Such effects have already been shown for a few cobalamin riboswitches in D. hafniense [301],

D. mccartyi strain 195, another corrinoid-auxotroph, requires a concentration of $25 \mu \mathrm{g} / \mathrm{L}$ cyanocobalamin to support optimal TCE dechlorination rates and growth yield [324], a value that is similar to what was observed for D. restrictus. Therefore, and in addition to the ecogenomic biomarkers defined by Maphosa et al. [13], one could consider the physiological threshold of corrinoid concentration as a possible diagnostic tool to delineate the reductive dechlorination potential by corrinoid-auxotrophic OHRB in anaerobic environments. 
Meanwhile, the production of the PCE reductive dehalogenase (PceA, Dehre_2398) in D. restrictus showed no significant change under different corrinoid concentrations, which strongly suggests that the amount of available corrinoid and not of the apo-enzyme represents the main limiting factor for PCE dechlorination.

Taken altogether, our results support the hypothesis that, besides the partial deletion of $c b i H$ in $D$. restrictus $[67,261]$, which already represents a crucial checkpoint in the corrinoid biosynthesis pathway, the energetic cost of de novo corrinoid biosynthesis might explain why $D$. restrictus has developed enhanced corrinoid transport and salvaging strategies. $D$. restrictus corrinoid metabolism represents an intermediate situation between the true corrinoid-auxotrophic and obligate organohalide-respiring D. mccartyi, which lacks the corrinoid biosynthesis pathway completely [93, 300, 324, 325], and the facultative OHRB able to produce corrinoids de novo. 


\section{Acknowledgments}

The Swiss National Science Foundation (SNSF) is acknowledged for support to Aamani Rupakula, Julien Maillard and Christof Holliger in frame of the SNF project No. 31003A_138114.We furthermore thank the European Community program FP7 (grants KBBE-211684; BACSIN, and KBBE-222625; METAEXPLORE) for financial support to

Hauke Smidt and Thomas Kruse, and the China Scholarship Council for support to Yue Lu. 


\title{
Dehalogenation of three
}

\section{tetrachlorobenzene isomers by}

\section{contaminated harbor sludge-derived}

\author{
enrichment cultures follows
}

\section{thermodynamically favorable reactions}

This chapter has been submitted for publication: Yue Lu, Javier Ramiro-Garcia, Pieter Vandermeeren, Steffi Herrmann, Danuta Cichocka, Dirk Springael, Siavash Atashgahi and Hauke Smidt. Dechlorination of three tetrachlorobenzene isomers by contaminated harbor sludge-derived enrichment cultures follows thermodynamically favorable reactions. Under review. 


\begin{abstract}
Dechlorination patterns of the three tetrachlorobenzene isomers 1,2,3,4-, 1,2,3,5- and 1,2,4,5TeCB were studied in anoxic microcosms derived from a contaminated harbor. The removal of doubly, singly and un-flanked chlorine atoms was noted in 1,2,3,4- and 1,2,3,5-TeCB fed microcosms, whereas only singly flanked chlorine was removed in 1,2,4,5-TeCB microcosms. Di- and/or mono-chlorobenzene were observed as the main end products of the reductive dechlorination of all three isomers. Among various dechlorinating pathways of TeCB isomers, the thermodynamically more favorable reactions were selectively followed by the enriched cultures. Based on quantitative PCR analysis targeting 16S rRNA genes of known organohalide-respiring bacteria, the growth of Dehalococcoides was found to be associated with the reductive dechlorination of all three isomers, while growth of Dehalobacter, another known TeCB dechlorinator, was only observed in one 1,2,3,5-TeCB enriched microcosm among biological triplicates. Numbers of Desulfitobacterium and Geobacter as facultative dechlorinators were rather stable suggesting that they are not directly involved in $\mathrm{TeCB}$ dechlorination. Overall bacterial community profiling suggested bacteria belonging to the phylum Bacteroidetes and the order Clostridiales as well as sulfate-reducing members of the class Deltaproteobacteria as putative stimulating guilds that provide electron donor and/or organic cofactors to fastidious dechlorinators. Overall, our results provide a better understanding of thermodynamically preferred $\mathrm{TeCB}$ dechlorinating pathways in harbor environments and microbial guilds enriched and active in anoxic TeCB dechlorinating microcosms.
\end{abstract}




\section{Introduction}

Chlorobenzenes (CBs) are aromatic chlorinated compounds with a benzene ring substituted with one to six chlorine atoms. CBs are important industrial intermediates in pesticides synthesis and manufacturing of other chemicals [326, 327]. Anthropogenic production and uncontrolled release of $\mathrm{CBs}$ in addition to their high persistence and bioaccumulation has resulted in broad distribution of these pollutants in the environment [326-328]. Partial dechlorination of highly chlorinated benzenes has been shown in a broad range of suboxic/anoxic environments such as soil [329, 330], river sediment [331-333], sewage sludge [334, 335] and drainage ditch [57] with lower chlorinated benzenes as end products. Even though monochlorobenzene (MCB) was considered to be recalcitrant to biotransformation in anoxic environments [8], several studies reported further dechlorination of MCB to benzene in anaerobic enrichments originated from freshwater sediments[333, 336, 337]. Organohalide respiration (OHR), i.e. energy conservation from respiratory reductive dechlorination of organohalides as the final electron acceptors, is currently the only known microbial process for the partial/complete dechlorination of highly chlorinated benzenes like hexachlorobenzene (HCB), pentachlorobenzene (QCB) and tetrachlorobenzenes (TeCBs) under anoxic conditions $[8,338]$. Therefore, the presence and activity of organohalide-respiring bacteria (OHRB) is pivotal for bioremediation of anoxic $\mathrm{CB}$ contaminated environments.

TeCBs have previously been reported as intermediates of HCB dechlorination, and include three isomers, i.e., 1,2,3,4-, 1,2,3,5- and 1,2,4,5-TeCB. TeCBs can be anaerobically biotransformated following diverse pathways [8, 338]. Preference for thermodynamically more favorable $\mathrm{CB}$ dechlorination pathways was shown before in an anaerobic microbial consortium originating from lake sediment [339], but this selectivity was not observed in other microcosms derived from contaminated freshwater sediments [336, 340]. Currently known bacterial isolates able to dechlorinate TeCB isomers belong to Dehalococcoides mccartyi in the phylum Chloroflexi $[159,335]$ and Dehalobacter in the phylum Firmicutes [57]. D. mccartyi strains CBDB1 [159], 195 [335] and DCMB5 [27] dehalogenate CB congeners with four or more chlorine substitues to trichlorobenzenes (TCBs) or dichlorobenzenes (DCBs). Besides, a broad TeCB dechlorination spectrum was demonstrated for Dehalobacter spp. strains 12DCB1 and 13DCB1 and a highly enriched culture containing Dehalobacter sp. 14DCB1, all of which were able to dechlorinate highly chlorinated CBs to DCBs and MCB [57]. In addition to these obligate organohalide-respiring isolates that are restricted to OHR as the sole metabolism to yield energy, growth of members of metabolically 
more versatile OHRB in the presence of CBs was also studied. Bacteria of the genus Geobacter were found associated with $\mathrm{HCB}$ dechlorination in an anaerobic consortium originating from contaminated river sediment as determined by genus-specific quantitative PCR (qPCR), suggesting they also may serve as CB dechlorinators [336]. Furthermore, a recently reported bacterial community analysis with 454 pyrosequencing of $16 \mathrm{~S}$ ribosomal RNA (rRNA) gene fragments conducted on a range of anaerobic CB-dechlorinating enrichments from contaminated river sludge revealed a high relative abundance $(21.38 \%$ $55.07 \%$ ) of populations closely related to Desulfitobacterium, while Dehalococcoides and Dehalobacter together represented less than $0.53 \%$ of the bacterial community [340].

Up to date, the fate of TeCBs in marine and estuarine environments is still not well known. 1,2,4-TCB dechlorination in sediment collected from Ise Bay (Japan) revealed that DCB production rates were related to anaerobic plate counts and activity of sulfate reducing bacteria, although there was no solid evidence showing their direct involvement in 1,2,4-TCB dechlorination [341]. Furthermore, dechlorination of CBs was reported in enrichments derived from estuarine sediments collected in Tsurumi river (Japan) [342] and Bayou d'Inde (USA) [343], but the microorganisms responsible for dechlorination were not identified. To date, the only reported $\mathrm{CB}$-dechlorinating bacterium isolated from an estuarine environment (Charleston Harbor, USA) is Dehalobium chlorocoercia [40], which was shown to dechlorinate $\mathrm{HCB}$ via $1,2,3,5-\mathrm{TeCB}$ to $1,3,5-\mathrm{TCB}$ [115]. This pathway was also proposed as the main pathway of dechlorination of $\mathrm{HCB}$ and QCB in estuarine sediments with very low levels of other CBs detected [115, 343]. Overall, dechlorination of CBs and in particular of $\mathrm{TeCB}$ in marine and estuarine sediments remains poorly explored, and better understanding of the activity of $\mathrm{CB}$-dehalogenating microbes and the microbial context they interact with in those habitats is required for their in situ bioremediation [344].

The aim of this study was to assess i) the $\mathrm{TeCB}$ dechlorination potential of microbial communities present in an estuarine environment ii) microbial community dynamics during enrichment and iii) the preferred TeCB dechlorinating pathways. To this end, we characterized TeCB-fed anoxic microcosms derived from contaminated harbor sludge, and a combilation of bacterial community profiling and $\mathrm{qPCR}$ were applied to gain insight into TeCB dechlorinators and co-existing, non-dechlorinating bacterial guilds that may be important for sustained dechlorination by providing electron donors and/or organic cofactors required by $\mathrm{OHRB}$. 


\section{Materials and methods}

\section{Chemicals}

CBs, benzene, and 1,3,5-tribromobenzene $(\mathrm{TBrB})$ of analytical grade and high purity were purchased from Sigma Aldrich. Benzene, MCB, 1,2-DCB, 1,4-DCB, 1,2,3-TCB, 1,2,4-TCB, $1,3,5-\mathrm{TCB}$ and $1,2,3,5-\mathrm{TeCB}$ had a purity of $99 \% ; 1,3-\mathrm{DCB}, 1,2,4,5-\mathrm{TeCB}$ and 1,3,5-TBrB: 98\%; and 1,2,3,4-TeCB: $96 \%$.

\section{Microcosm Setup}

Harbor sludge from Zeebrugge, Belgium was sampled (Figure 5.1, step 1) to prepare anoxic microcosms. All samples were collected in September 2008, and stored for three months at 4 ${ }^{\circ} \mathrm{C}$ until they were used in this study. The microcosms were set up as described elsewhere [340] with $10 \mathrm{~g}$ of sludge and $40 \mathrm{ml}$ of an anoxic medium [52], and $20 \mathrm{mM}$ lactate as electron donor and carbon source (Figure 5.1, step 2). Three TeCB isomers (1,2,3,4-, 1,2,3,5- and $1,2,4,5-\mathrm{TeCB})$ were added individually from acetone stocks to final concentrations of $50 \mu \mathrm{M}$ for each isomer. Abiotic controls were prepared by addition of $0.5 \mathrm{ml}$ of $37 \%$ formaldehyde. Initial microcosms were set up in triplicates. Two spikes of $50 \mu \mathrm{M}$ of $\mathrm{TeCB}$ isomer were supplied separately to the cultures during 93 days of non-shaking cultivation at $25{ }^{\circ} \mathrm{C}$ in the dark (Figure 5.1, step 2). Cultures were subsequently kept for five years at $4{ }^{\circ} \mathrm{C}$, after which a subset (single 1,2,3,4- and 1,2,4,5-TeCB enrichment cultures and triplicate 1,2,3,5-TeCB cultures) was transferred to Wageningen, the Netherlands, for further characterization of TeCB dechlorination. To revive TeCB dechlorination in the original cultures, $30 \mathrm{ml}$ of fresh anoxic medium was added to the original bottles and $20 \mathrm{mM}$ lactate was supplied. Bottles were sealed with a viton stopper, and the headspace was exchanged with $\mathrm{N}_{2} / \mathrm{CO}_{2}(80: 20 \mathrm{v} / \mathrm{v})$. Spikes of the respective TeCBs $(50 \mu \mathrm{M} /$ each) were added at the beginning and on day 35, 96 and 134 of incubation, and the cultures were maintained actively dechlorinating for over 200 days without shaking at $25^{\circ} \mathrm{C}$ in the dark (Figure 5.1, step 3).

To study the dynamics of degradation pathways and microbial communities, and to obtain sediment-free TeCB-enriched cultures, serial transfers were performed. A 5\% inoculum from the original TeCB-fed microcosms was transferred into fresh anoxic bottles containing growth medium, fed with $50 \mu \mathrm{M}$ of the same TeCB isomer as the initial inoculum and cultivated for 66 days (Figure 5.1, step 4). Further transfers were conducted with the 1,2,4,5-TeCB dechlorinating culture enriched from harbor sludge (culture A in Figure 5.1, step 5), and their performance was compared to that of transfers of two sediment-free $1,2,4,5$-TeCB-enriching 
cultures (culture B and C in Figure 5.1, step 5) that were previously obtained from chlorinated aromatics-contaminated river sludge collected in Kanaal Ieper-Ijzer, Belgium $\left(50^{\circ} 52^{\prime} 14^{\prime \prime} \mathrm{N}\right.$ $2^{\circ} 52^{\prime} 31^{\prime \prime} \mathrm{E}$ ) [340]. A 5\% inoculum was taken for serial transfer (Figure 5.1, step 6).

Effects of vancomycin addition and/or vitamin B12 starvation on the dechlorination activity of the sediment-free $1,2,4,5$-TeCB-fed enrichments were investigated with a $2 \times 2$ factorial design experiment (Figure 5.1, step 7) that was set up in duplicate. $12 \mu \mathrm{g} / 1$ of vancomycin and $0.03 \mu \mathrm{M}$ of vitamin B12 were added where required.

\section{Detection of benzene and chlorinated benzenes}

Benzene and CBs were quantified on a GC-2000 equipped with flame ion detector (FID) (Shimadzu, Japan). $0.2 \mathrm{ml}$ headspace samples were used for benzene measurement as described before [345], and $1 \mathrm{ml}$ liquid samples were used for the detection of CBs. Liquid samples were extracted with $400 \mu 1$ hexane by overnight shaking at $600 \mathrm{rpm}$ followed by 100 $\mu 1$ hexane phase transfer to HPLC vials containing $5 \mu 1$ of $1 \mathrm{mM} \mathrm{1,3,5-tribromobenzene} \mathrm{as} \mathrm{the}$ internal standard. The column temperature program was $60{ }^{\circ} \mathrm{C}$ hold for $4 \mathrm{~min}$, followed by a gradient of $5{ }^{\circ} \mathrm{C} \mathrm{min}-1$ to $180{ }^{\circ} \mathrm{C}$ and another hold for $3 \mathrm{~min}$. The carrier gas was nitrogen at a flow of $1 \mathrm{ml} \mathrm{min}$, a spit flow of $12 \mathrm{ml} \mathrm{min}^{-1}$ with a split ratio of 1:10. Injection was performed by an auto-sampler (Shimadzu, Japan) injecting $2 \mu 1$ of the sample into a split injector held at $250{ }^{\circ} \mathrm{C}$. Standards with a mixture of benzene and CBs at 10, 20, 40, 60, 100, $200 \mu \mathrm{M}$ were set up in $125 \mathrm{ml}$ serum bottles with the same headspace-liquid phase ratio of treatment cultures.

\section{Sampling for molecular analysis and DNA extraction}

Cultures were sampled for molecular analysis as shown in Figure 5.1, and samples taken before adding TeCBs to the original bottles were indicated as 'Before reviving' (Figure 5.1, step 3). Two $\mathrm{ml}$ slurry/liquid samples were withdrawn from each bottle, centrifuged (5417R, Eppendorf, Germany) at $6010 \mathrm{rcf}$ for $5 \mathrm{~min}$ at $4{ }^{\circ} \mathrm{C}$, and pellets were stored at $-20{ }^{\circ} \mathrm{C}$. DNA isolation was performed using the PowerSoil DNA isolation kit (MO-BIO, USA) according to the manufacturer's instructions except that three $45 \mathrm{~s}$ bead beating $(5.5 \mathrm{~m} / \mathrm{s})$ steps using a Fastprep Instrument (MP Biomedicals, USA) were included instead of the horizontally vortexing step suggested in the manual. The quality and quantity of DNA was checked using a Nanodrop 2000c Spectrophotometer (Thermo Fisher Scientific, USA) and agarose gel electrophoresis. 


\section{Illumina MiSeq sequencing and data analysis}

A 2-step PCR strategy was used to generate barcoded amplicons from the V1-V2 region of the 16S rRNA gene. The first PCR $(50 \mu \mathrm{l})$ contained $1 \times$ HF buffer (Thermo Scientific ${ }^{\mathrm{TM}}$, The Netherlands), $1 \mu$ dNTP Mix (10 mM; Promega, Leiden, The Netherlands), 1 U of Phusion ${ }^{\circledR}$ Hot Start II High-Fidelity DNA polymerase (Thermo Scientific ${ }^{\mathrm{TM}}$ ), $500 \mathrm{nM}$ of 27F-DegS forward primer, $500 \mathrm{nM}$ of 338R I and II reverse primers (Table S5.1), and $1 \mu \mathrm{L}$ template DNA (15-20 ng/ $\mu \mathrm{l})$. The forward and reverse primers were appended at the 5' end with $18 \mathrm{bp}$ Universal Tag (Unitag) 1 and 2, respectively (Table S5.1). PCR conditions were $98^{\circ} \mathrm{C}$ for $30 \mathrm{~s}$, 25 cycles of denaturation at $98^{\circ} \mathrm{C}$ for $10 \mathrm{~s}$, annealing at $56^{\circ} \mathrm{C}$ for $20 \mathrm{~s}$ and elongation at $72^{\circ} \mathrm{C}$ for $20 \mathrm{~s}$, and a final extension at $72^{\circ} \mathrm{C}$ for $10 \mathrm{~min}$. The PCR product size was examined by gel electrophoresis. The second PCR $(100 \mu \mathrm{l})$ contained $1 \times$ HF buffer, $2 \mu \mathrm{l}$ dNTP Mix $2 \mathrm{U}$ of Phusion ${ }^{\circledR}$ Hot Start II High-Fidelity DNA polymerase, $500 \mathrm{nM}$ of a forward and reverse primer equivalent to the Unitag1 and Unitag2 sequences, respectively, that were each appended with an $8 \mathrm{nt}$ sample specific barcode [346] at the 5' end, and $5 \mu$ PCR product of the first reaction. PCR conditions were similar to those used for the first PCR except for an annealing temperature of $52^{\circ} \mathrm{C}$ and a reduced number of 5 cycles. The PCR product size was examined by gel electrophoresis, purified with HighPrep ${ }^{\mathrm{TM}}$ (Magbio Genomics, Rockville, MD, USA) and quantified using a Qubit in combination with the dsDNA BR Assay Kit (Invitrogen, Carlsbad, CA, USA). Purified PCR products were pooled, underwent adaptor ligation and sequenced on a MiSeq platform (GATC-Biotech, Konstanz, Germany). Sequence analysis was performed with NG-Tax, an in-house pipeline [346] in which operational taxonomic units (OTUs) were assigned to taxonomy using uclust [347] in an open reference approach against the SILVA 16S rRNA gene reference database [348]. Finally a biom file was generated using Quantitative Insights Into Microbial Ecology (QIIME) v1.2 [349].

\section{Quantitative PCR}

The abundance of the $16 \mathrm{~S}$ rRNA genes of Dehalococcoides, Dehalobacter, Desulfitobacterium, Geobacter and total bacteria was determined by qPCR performed on a CFX384 Real-Time system with C1000 Thermal Cycler (Bio-Rad Laboratories, USA) with $\mathrm{iQ}^{\mathrm{TM}}$ SYBR Green Supermix (Bio-Rad Laboratories, USA). Each DNA sample was analysed in triplicates. The primers and qPCR amplification program used in this study have been described before for Dehalobacter [226] and other targets [352]. The relative abundance of Dehalococcoides in qPCR data was calculated by dividing the number of Dehalococcoides $16 \mathrm{~S}$ rRNA gene copies by the copy number of bacterial $16 \mathrm{~S}$ rRNA gene copies, multiplied by 
100.

\section{Nucleotide sequences}

16S rRNA gene sequences have been deposited in the European Nucleotide Archive (ENA) with accession number ERS1082969-ERS1083005 under study PRJEB13024.

\section{Results}

\section{TeCB dechlorination in sediment microcosms}

During 93 days of anaerobic cultivation (Figure 5.1, step 2), complete depletion of two spikes of $50 \mu \mathrm{M}$ of corresponding TeCBs $(1,2,3,4-, 1,2,3,5-, 1,2,4,5-\mathrm{TeCB})$ was observed in all sediment microcosms (data not shown). In contrast, no decrease of TeCBs was observed and no dechlorination products were detected in the abiotic controls (data not shown), indicating that biological dechlorination via OHR occurred.

After these microcosms had been stored for five years at $4{ }^{\circ} \mathrm{C}$, new spikes of TeCBs were added to recover the dechlorination activity. After a short lag phase of 1-8 days, dechlorination of TeCBs was observed in all microcosms (Figure 5.2). 1,2,4-TCB was observed as a transient intermediate during the dechlorination of all three TeCB isomers, whereas 1,3,5- TCB was only detected as an intermediate product of 1,2,3,5-TeCBdechlorination. All microcosms showed MCB accumulation except for the 1,2,4,5-TeCB-fed microcosm in which 1,4-DCB was detected as the main end product (Figure 5.2e). Production of 1,2,3-TCB, 1,2-DCB or benzene was not detected in any of the microcosms during 161 days of incubation.

\section{1,2,4,5-TeCB dechlorination in sediment-free cultures}

To achieve sediment-free TeCB-enriched cultures, a 5\% inoculum was directly transferred from original TeCB-fed microcosms (Figure 5.1, step 4). Dechlorination was only observed in the transferred 1,2,4,5-TeCB-fed enrichments (culture A in Figure 5.1, step 5) after 66 days of anaerobic cultivation (data not shown).

In the following, the dechlorination performance of the newly obtained transfer culture derived from the estuarine harbor environment was compared with another two sediment-free $1,2,4,5-\mathrm{TeCB}$-enriching cultures that were previously enriched from river sludge in Kanaal Ieper-Ijzer (Belgium) [340]. During incubation of the second transfer, three spikes of 1,2,4,5TeCB were completely depleted in all transferred enrichments, concomitant with the 


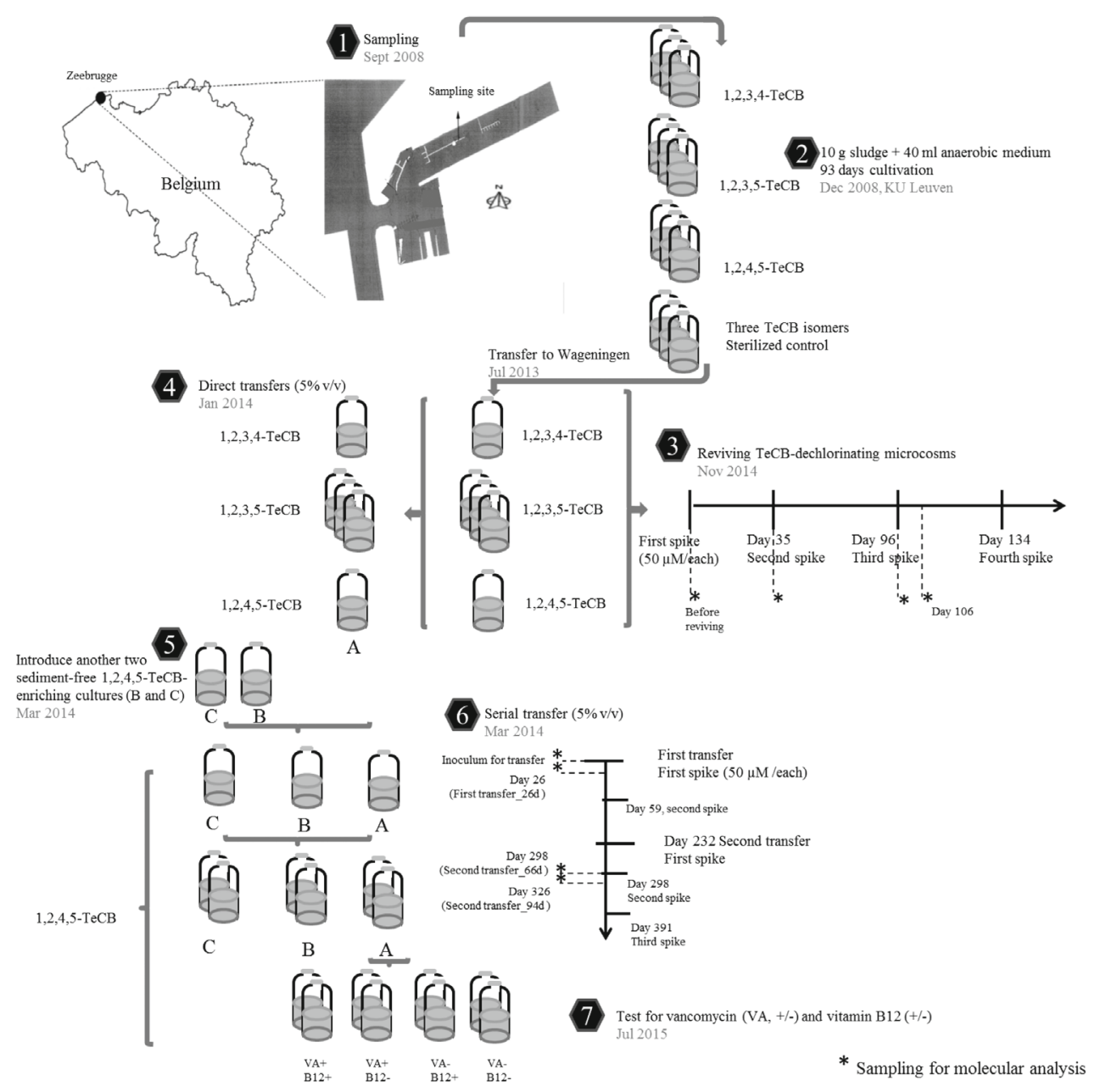

Figure 5.1 Schematic representation of the experiment.

production of 1,2,4-TCB, 1,4-DCB and 1,3-DCB (Figure 5.3). 1,4-DCB was the dominant end product in harbor derived enrichments (Figure 5.3a), which was in line with the observation in the original 1,2,4,5-TeCB-fed microcosm (Figure 5.2e), whereas slightly more 1,3-DCB was detected in river sludge-derived enrichments (Figure 5.3b\&c).

\section{Dechlorination pattern of TeCB isomers and selectivity of the reactions}

The reductive removal of doubly, singly and non-flanked chlorine atoms was noted in the 1,2,3,4- and 1,2,3,5-TeCB-fed microcosms derived from contaminated harbor sludge. In contrast only singly flanked, but not the non-flanked chlorine, was removed during the 


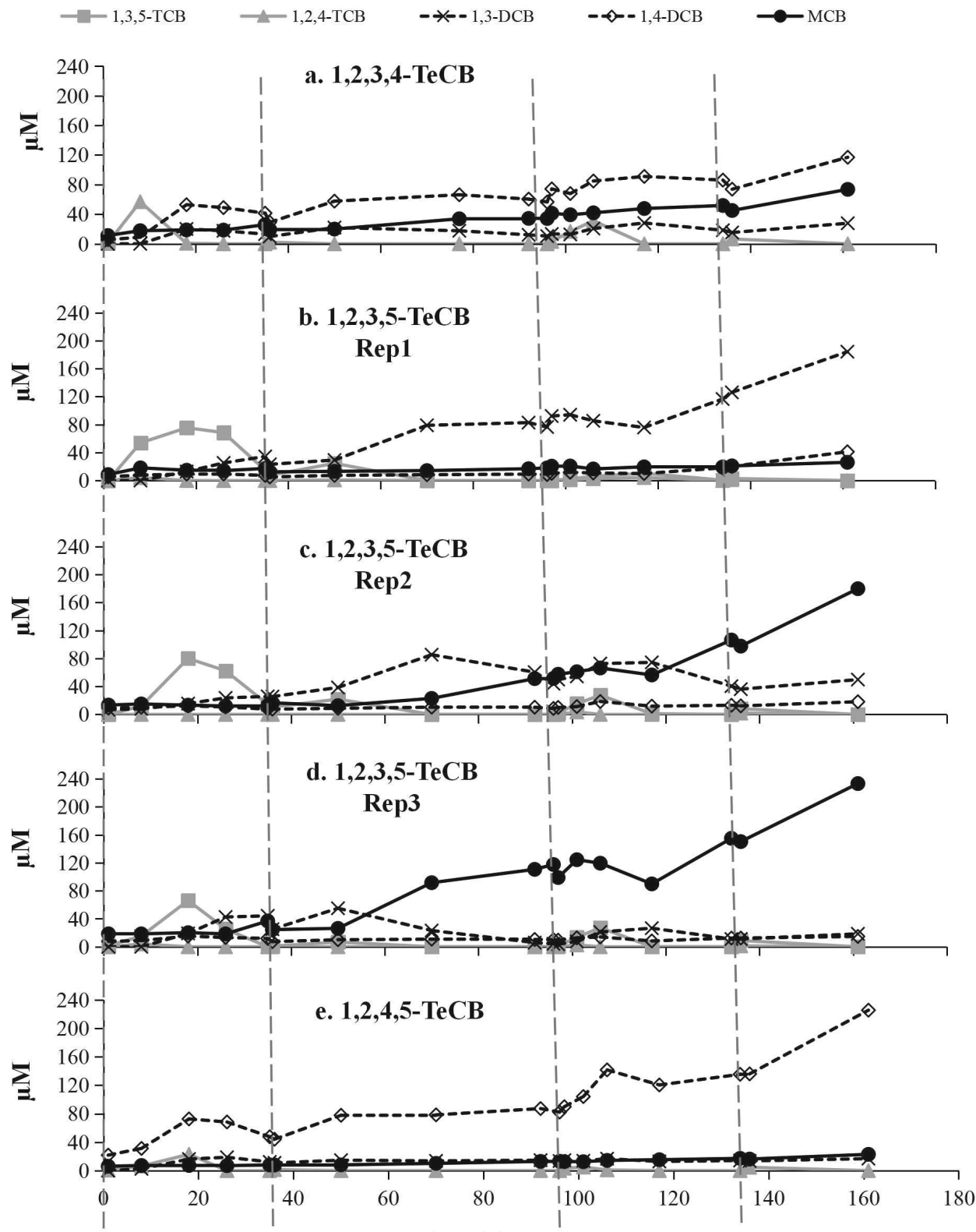

Time (d)

Figure 5.2 Dechlorination of 1,2,3,4-TeCB (a), 1,2,3,5-TeCB (b-d, triplicate bottles), and $1,2,4,5-\mathrm{TeCB}(\mathbf{e})$ in original microcosms derived from harbor sludge. $50 \mu \mathrm{M}$ spikes of the respective TeCBs are indicated by vertical dashed lines. Concentrations of TeCBs are not shown for the clarity of figure. 

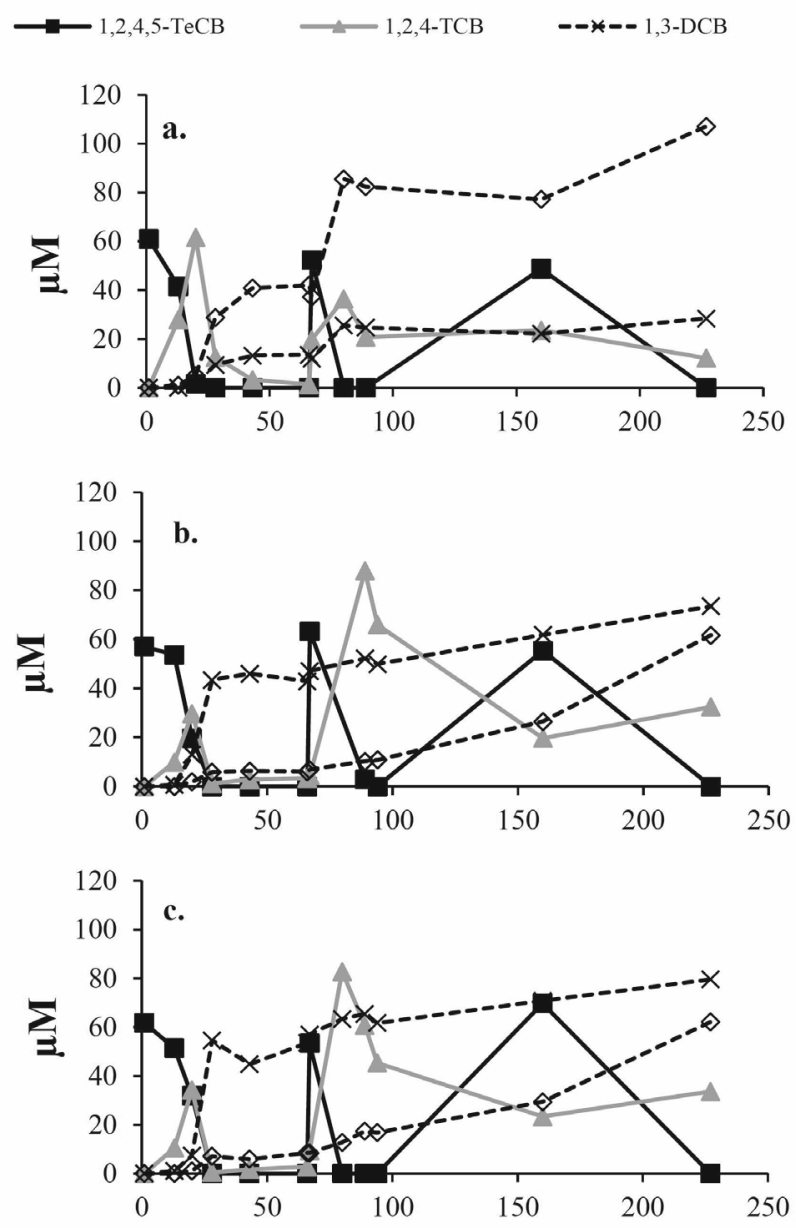

Time (d)

Figure 5.3 Dechlorination of 1,2,4,5-TeCB in second transfers (Figure 5.1, step 6) from the 1,2,4,5-TeCB dechlorinating enrichment derived from harbor sludge (a) and 1,2,4,5-TeCB dechlorinating sediment-free enrichments (culture B and C in Figure 5.1) derived from river sludge (b-c). Data from an individual bottle is shown here as a representative of duplicate cultures, which both followed the same overall dechlorination pattern.

dechlorination of 1,2,4,5-TeCB. Based on Gibbs free-energies ( $\Delta \mathrm{G}^{0 \prime}$ under standard condition, $\mathrm{kJ} / \mathrm{mol}$ ) taken from Dolfing and Keith Harrison [353] calculated with hydrogen as electron donor, the harbor sludge-derived microcosms showed a preference to follow the thermodynamically most favorable dechlorination pathways. For example, 1,2,3,5-TeCB was mainly dechlorinated to $1,3,5-\mathrm{TCB}$ as an intermediate rather than 1,2,4-TCB, and 1,2,3-TCB was not detected, in line with the fact that this reaction theoretically yields the least negative 
free energy change (Figure 5.4). A similar pattern was observed for the dechlorination of $1,2,3,4-\mathrm{TeCB}$ to $1,2,4-\mathrm{TCB}$ instead of $1,2,3-\mathrm{TCB}$ and further dechlorination of 1,2,4-TCB to 1,4-DCB instead of 1,3-DCB as the main product (Figure 5.4).

\section{Effect of vancomycin addition and vitamin B12 starvation}

Vancomycin, an inhibitor of the peptidoglycan synthesis of Gram-positive bacteria, was added to harbor sludge-derived, sediment-free 1,2,4,5-TeCB enrichments to test its effect on TeCB dechlorination. After 21 days of anaerobic cultivation, the amended 1,2,4,5-TeCB was completely depleted in control microcosms (Figure S5.1a) with 1,4-DCB and 1,3-DCB as the main products. In contrast, in vancomycin treated microcosms, only $36-59 \%$ of the $1,2,4,5-$ TeCB was dechlorinated within the same incubation time, with 1,2,4-TCB as the main product and trace amounts of 1,4-DCB and 1,3-DCB (Figure S5.1b). The delayed dechlorination in enrichments supplied with vancomycin suggested (in)direct involvement of vancomycin-sensitive Gram-positive bacteria in 1,2,4,5-TeCB dechlorination. Additionally, vitamin B12 (cofactor of reductive dehalogenase enzymes) starvation was investigated in the presence or absence of vancomycin (Figure S5.1c\&d) No effect, however, on TeCB dechlorination activity was observed, suggesting that the 1,2,4,5-TeCB-enriched microbial communities tested here were capable of de novo vitamin B12 biosynthesis.

\section{Dynamics of bacterial communities}

During 161 days reviving of original TeCB microcosms, Dehalococcoides as a potential $\mathrm{TeCB}$ dechlorinator was detected at relative abundances below $2 \%$ in 1,2,3,4- and 1,2,4,5TeCB fed microcosms and one of the triplicate 1,2,3,5-TeCB fed microcosms (Figure 5.5a). The detected sequence of Dehalococcoides was blast in NCBI and showed $100 \%$ identity with that of D. mccartyi strains CBDB1 and 195. Additionally, diverse non-dechlorinating bacteria were found in the bacterial community of TeCB-fed microcosms, especially from the phyla Bacteroidetes, Fibrobacteres and the Deltaproteobacteria class (Figure 5.5a). Bacteroidetes and Deltaproteobacteria were detected in all the microcosms in the range of $10-56 \%$ and 10 $47 \%$, respectively. Fibrobacteres was mainly enriched in the 1,2,4,5-TeCB-fed microcosm and two of three replicate 1,2,3,5-TeCB fed microcosms (Figure 5.5a), whereas Bacteroidetes was observed with higher relative abundance in the other 1,2,3,5-TeCB fed microcosm with no Fibrobacteres detected during cultivation (Figure 5.5a). Differences of bacterial community composition observed among three replicate 1,2,3,5-TeCB-fed microcosms may have been caused by the heterogeneity of inocula sediments. 
<smiles>Clc1ccc(Cl)c(Cl)c1Cl</smiles><smiles>Clc1cc(Cl)c(Cl)c(Cl)c1</smiles><smiles>Clc1cc(Cl)c(Cl)cc1Cl</smiles><smiles>[13CH3]</smiles><smiles>[104In]</smiles>

$$
1,2,4,5-\mathrm{TeCB}
$$

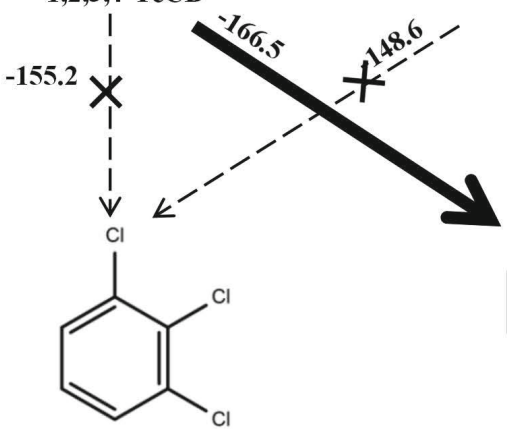<smiles>Clc1ccc(Cl)c(Cl)c1</smiles>

$\bullet$

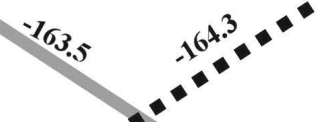

1,2,3-TCB

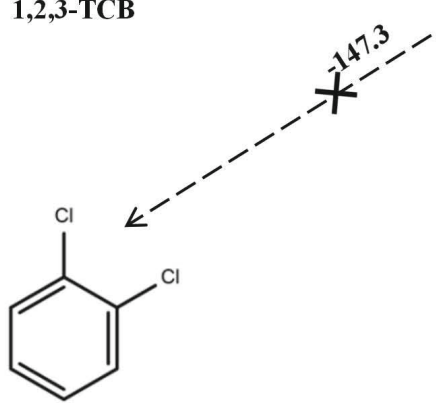

1,2-DCB
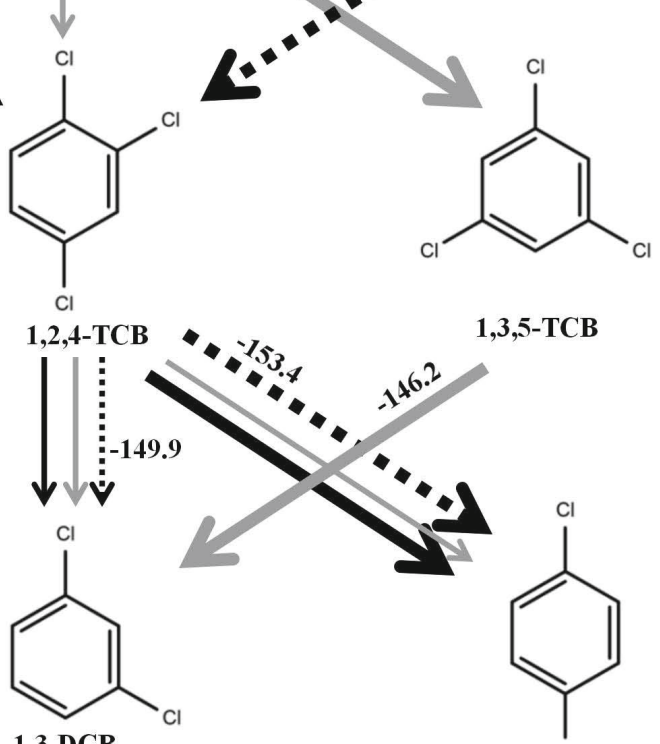

$1,3,5-T C B$
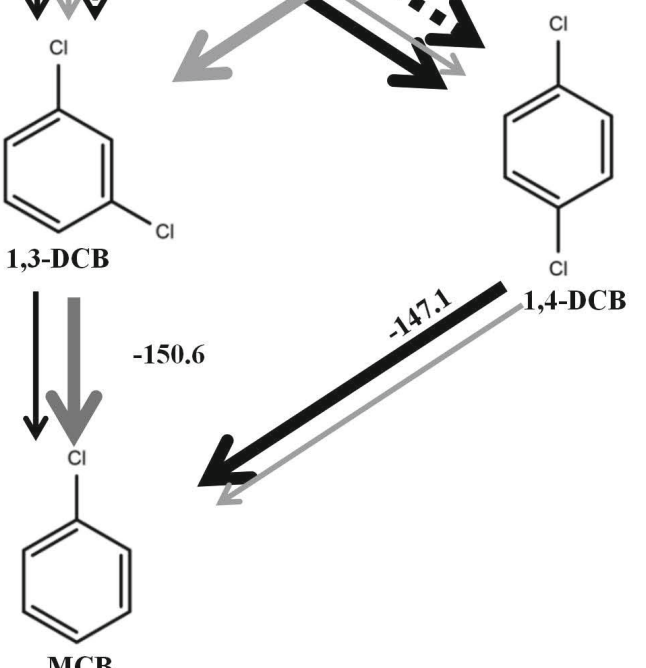

Figure 5.4 Dechlorination patterns of 1,2,3,4- (black solid line), 1,2,3,5- (grey solid line) and 1,2,4,5- (black dotted line) TeCB in microcosms derived from harbor sludge. Thin arrows represent reactions that were detected but were not dominant. Reactions catalyzed preferentially are indicated with bold arrows, and reactions not observed in our study are indicated by dashed lines with a cross. The $\Delta \mathrm{G}^{0 \prime}(\mathrm{kJ} / \mathrm{mol})$ indicated for each dechlorination reaction is based on previously reported calculations with hydrogen as the electron donor [353]. 
a.

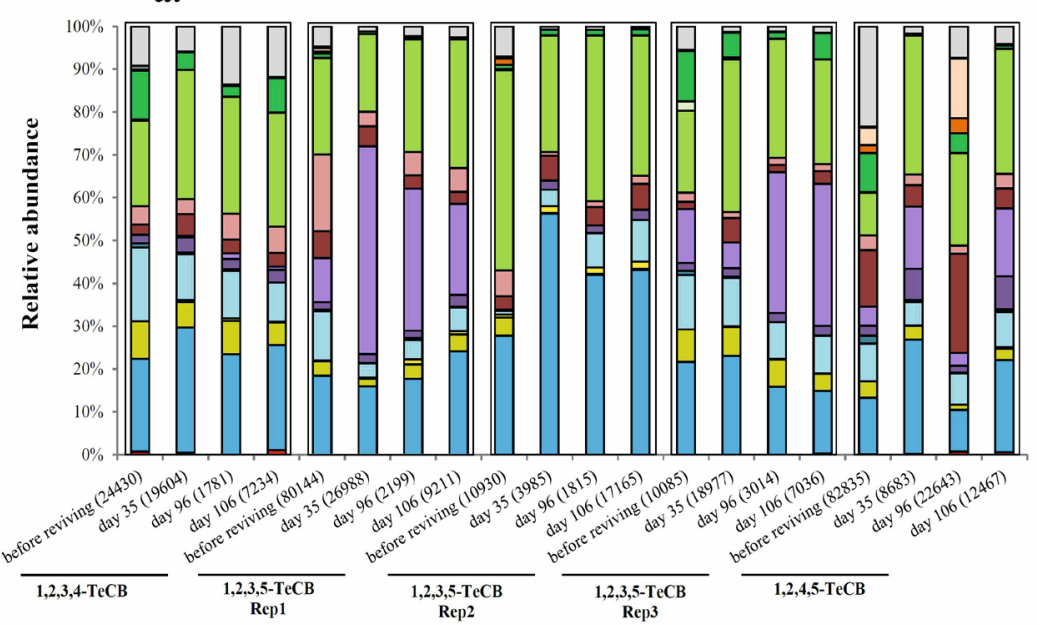

b.

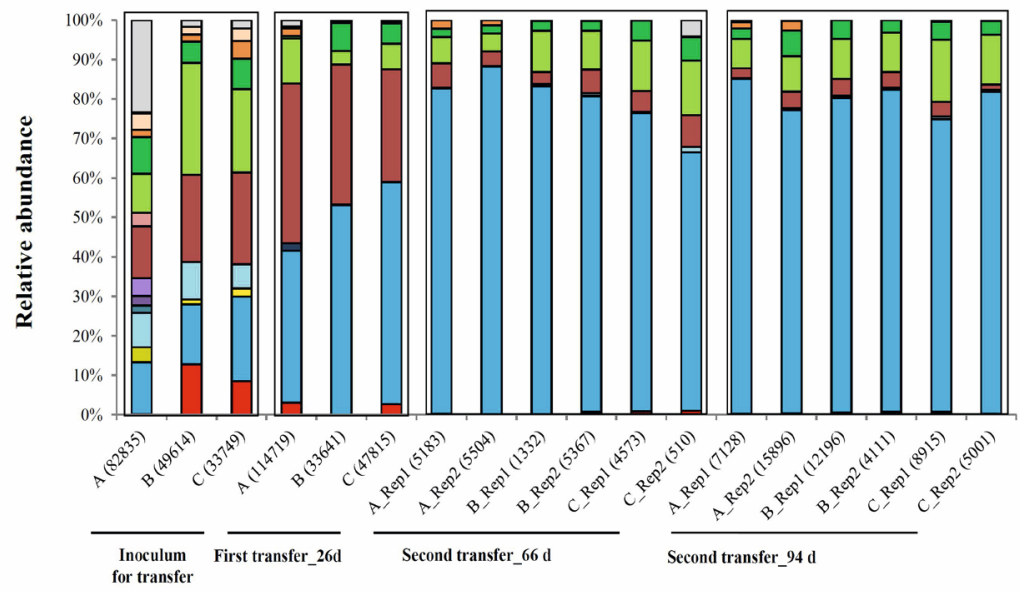

口Non-assigned

口Others

口Thermotogae

口Synergistetes

口Spirochaetes

口Gammaproteobacteria

口Deltaproteobacteria

口Nitrospirae

口Firmicutes

$\square$ Fibrobacteres

口Deferribacteres

-Cyanobacteria

口Chloroflexi(not Dhc)

口Chlorobi

口Candidate_division_OP9

-Candidate division_ODI

口Bacteroidetes

口Dehalococcoides (Dhc)

Figure 5.5 Bacterial 16S rRNA gene-based community dynamics of TeCB-fed microcosms during 161 days of reviving (a) and 1,2,4,5-TeCB dechlorinating enrichments during serial transfers (b). a. Rep1, Rep2 and Rep3 are triplicate original bottles fed with 1,2,3,5-TeCB. Sampling times are labelled as in Figure 5.1 (step 3). b. Rep1 and 2 are duplicates. Inoculum and sampling time are labeled as in Figure 5.1 (step 6). Data are shown at phylum level, except Delta- and Gammaproteobacteria that are presente at the class level and the genus Dehalococcoides that is shown separately from its corresponding phylum (Chloroflexi). The number of reads of each sample is given in brackets. Phyla with relative abundance lower than $1 \%$ in all samples are summed up as 'Others'. 
During serial transfers of the different 1,2,4,5-TeCB-enriched cultures, members of the Bacteroidetes phylum became strongly enriched with an average relative abundance of $79.7 \%$ $(\mathrm{SD}=3.8$, Table $\mathrm{S} 5.2)$ at the end of the second transfer (Figure 5.5b). Bacteroidetes detected in the the MiSeq dataset were mainly from the classes Bacteroidia (especially from the genus Petrimonas) and Sphingobacteriia (Table S5.2). Petrimonas was not detected in the TeCBenriched cultures derived from harbor sludge, in which the Sphingobacteriia dominated the bacterial community (Table S5.2).

\section{Quantification of potential dechlorinators during TeCB dechlorination}

Growth of total bacteria, as measured by bacterial 16S rRNA gene-specific qPCR, was observed in all TeCBs fed microcosms (Figure 5.6). Interestingly, in one out of three 1,2,3,5TeCB fed microcosms, the abundance of the genus Dehalobacter increased considerably from none-detectable levels before reviving to $2.59 \mathrm{E}+06(\mathrm{SD}=3.48 \mathrm{E}+05) 16 \mathrm{~S}$ rRNA gene copies/ml rRNA genes with $7.41 \mathrm{E}+04$ copies $/ \mathrm{ml}(\mathrm{SD}=1.74 \mathrm{E}+04)$. This indicates that in this microcosm, Dehalobacter rather than Dehalococcoides is the main TeCB dechlorinator. In all other microcosms, a clear increase of the Dehalococcoides counts during reviving was observed with an average abundance in the range of $3.93 \mathrm{E}+05$ to $1.12 \mathrm{E}+07$ copies $/ \mathrm{ml}$ at the end of the cultivation (Figure 5.6). In contrast, 16S rRNA gene copy numbers of Desulfitobacterium and Geobacter remained stable (Figure 5.6). In all the 1,2,4,5-TeCB-fed transferred cultures, Dehalococcoides was detected with abundances that were more than three orders of magnitude higher than those of Dehalobacter (Figure S5.2), suggesting a more important role of Dehalococcoides in 1,2,4,5-TeCB dechlorination in these enrichments. Furthermore, the relative abundance of Dehalococcoides calculated from qPCR data generally matched with the results derived from the MiSeq dataset, although in several samples higher relative abundance of Dehalococcoides was detected via MiSeq (Figure 5.7). Both qPCR and MiSeq results showed decreased relative abundance of Dehalococcoides during transfer, which was mainly caused by the increase of average bacterial numbers from $3.05 \mathrm{E}+08$ copies $/ \mathrm{ml}$ in inoculum to $1.98 \mathrm{E}+09$ copies/ml at 94 day of second transfer, while the absolute numbers of Dehalococcoides remained around 1.0E +07 copies $/ \mathrm{ml}$ medium during serial transfer (Figure S5.2). 


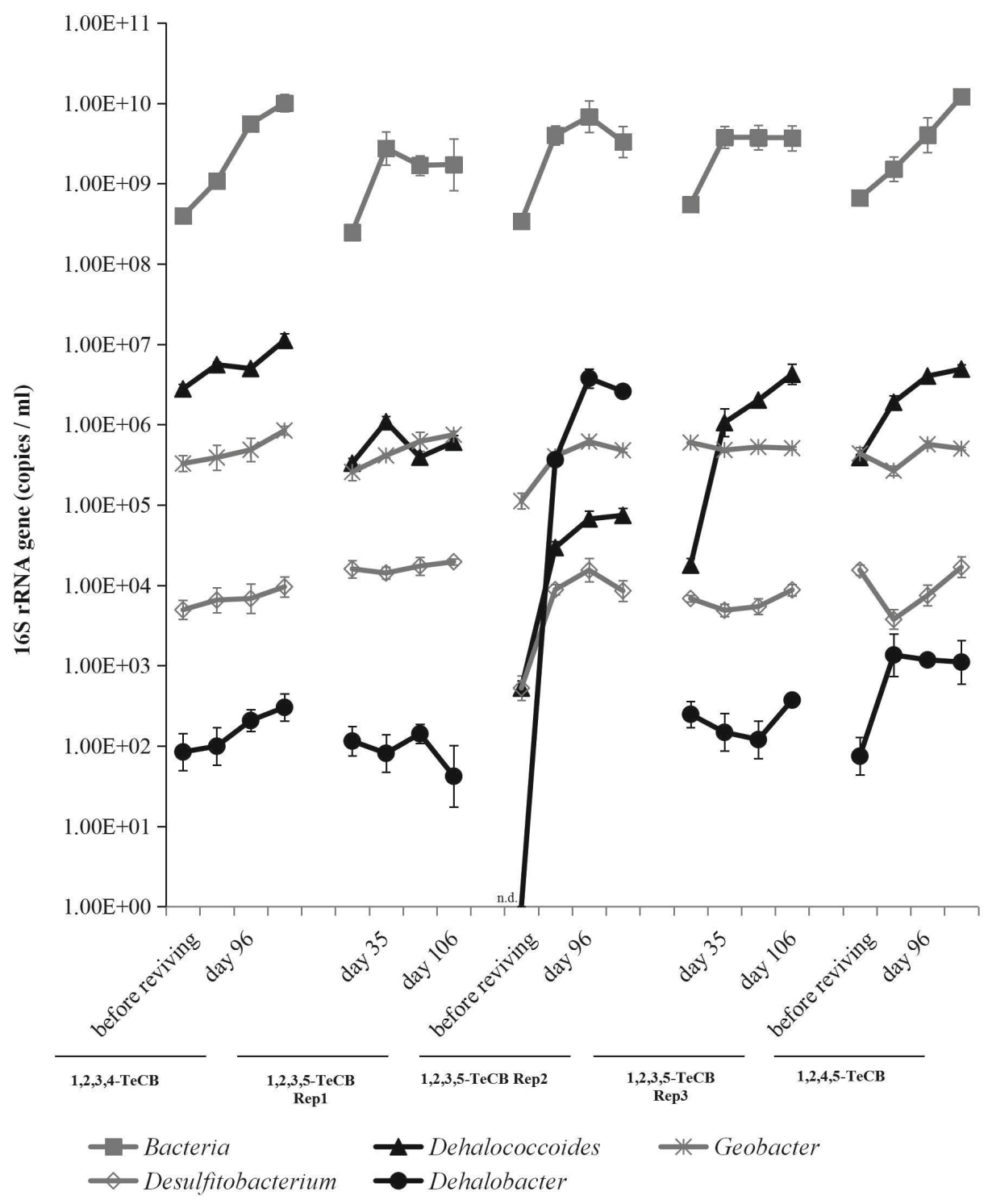

Figure 5.6 Quantitative PCR (qPCR) targeting 16S rRNA genes of Dehalobacter, Desulfitobacterium, Geobacter and Dehalococcoides, and of total bacteria during 161 days reviving of TeCB-fed microcosms derived from harbor sludge. Microcosm set up and sampling times are labelled as Figure 5.1 (step3). Rep1, Rep2 and Rep3 are triplicate original bottles fed with 1,2,3,5-TeCB. Error bars indicate standard deviations of qPCR technical triplicates. n.d. nondetectable. 

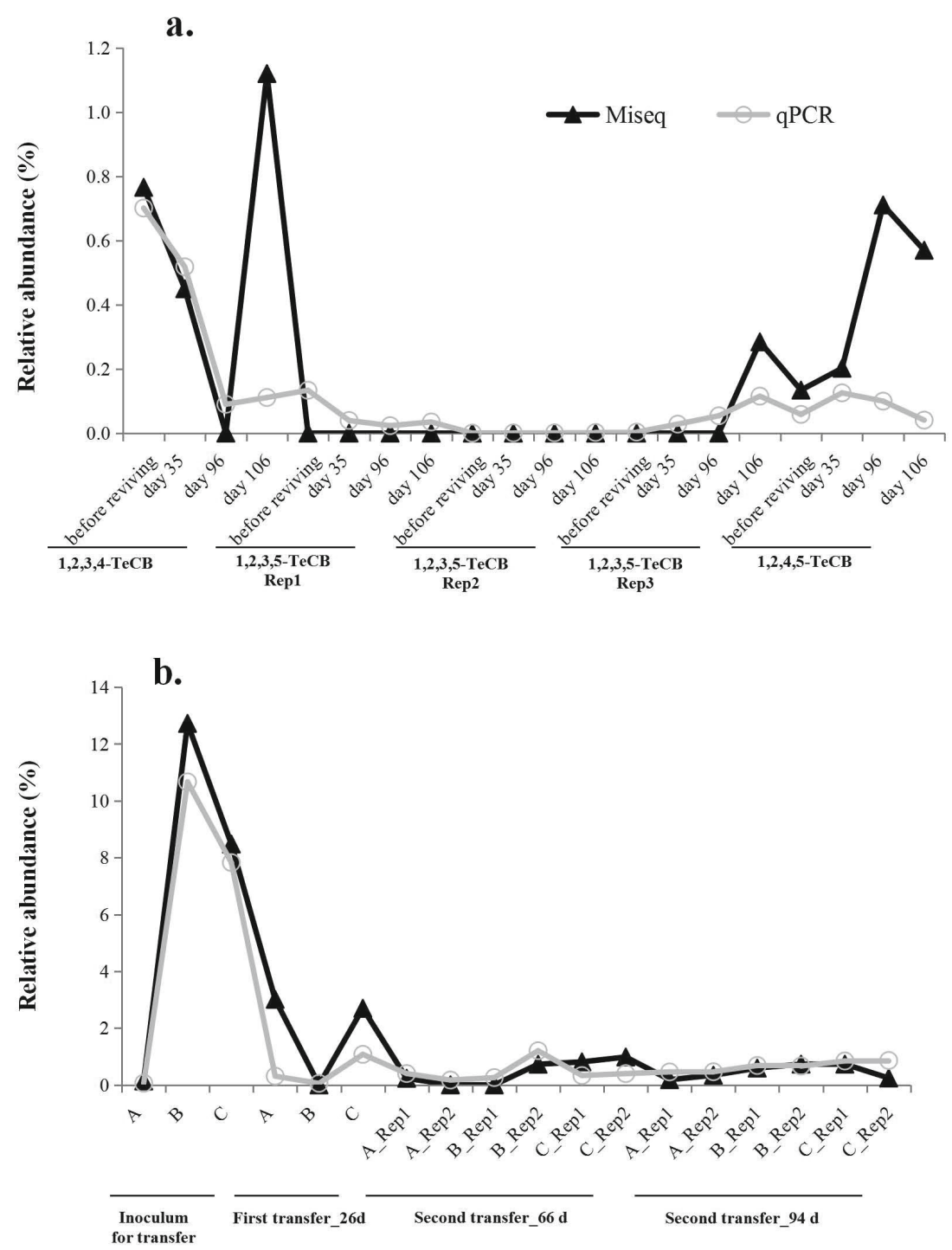

Figure 5.7 Relative abundance of Dehalococcoides in TeCB-fed microcosms during 161 days of reviving (a) and 1,2,4,5-TeCB dechlorinating enrichments during serial transfers (b). a. Rep1, Rep2 and Rep3 are triplicate original bottles fed with 1,2,3,5-TeCB. Sampling times are labelled the same as Figure 5.1 (step 3). b. Rep1 and 2 are duplicates. Inoculum and sampling time are labeled as in Figure 5.1 (step 6). 


\section{Discussion}

Dechlorination of $1,2,3,4-, 1,2,3,5-$ and $1,2,4,5-\mathrm{TeCB}$ isomers to $\mathrm{DCB}$ and/or MCB was observed in anoxic microcosms derived from harbor sludge, and enriched consortia showed selectivity in mediating thermodynamically more favorable steps in the reductive dechlorination of TeCB isomers (Figure 5.4). Such preference for thermodynamically more favorable $\mathrm{CB}$ dechlorination pathways was shown before in an anaerobic microbial consortium originating from lake sediment [339], but in that report, the TeCB isomers were dechlorinated with $1,3,5-\mathrm{TCB}$ or $1,2,4-\mathrm{TCB}$ as the end products. In our study, 1,2,3,5-TeCB was mainly dechlorinated to $1,3,5-\mathrm{TCB}$ with the removal of doubly flanked chlorine, and subsequent removal of the unflanked meta-substituted chlorines to produce 1,3-DCB (Figure $5.2 \mathrm{~b}$ ) and MCB (Figure 5.2c\&d). This implies that the enriched consortia from harbor sludge may host mutiple $\mathrm{CB}$ dechlorinators with complementary dechlorination function. The dechlorination of $\mathrm{HCB}$ via $1,2,3,5-\mathrm{TeCB}$ was found to predominate in cultures derived from estuarine sediment with 1,3,5-TCB [115] and 1,3-DCB [343] as the end products. Additionally, 1,3,5-TCB production from 1,2,3,5-TeCB was reported before for D. mccartyi strains $[27,159,335]$, however, no further dechlorination of 1,3,5-TCB was observed with those isolates and the underlying reason is still not clear. A different dechlorination pattern of 1,2,3,5-TeCB, with even less favourable thermodynamics, was found in Dehalobacter sp. 13DCB1, which predominantly dechlorinated singly flanked ortho-substituted chlorine with $1,2,4-\mathrm{TCB}$ as the dominant intermediate leading to the formation of 1,4-DCB as the main end product and small amounts of MCB [57]. 1,2,4-TCB and 1,4-DCB were also detected in our 1,2,3,5-TeCB amended microcosms, albeit not as dominant dechlorination products (Figure 5.4). Up to date, the dechlorination of $1,2,3,5-\mathrm{TeCB}$ with the removal of unflanked chlorine to produce 1,2,3-TCB has not been reported.

1,2,3-TCB was shown to be the only product of 1,2,3,4-TeCB dechlorination by Dehalobacter strain 12DCB1 and 14DCB1 [57] and could be further dechlorinated to 1,3-DCB by $D$. mccartyi strain DCMB5 [27]. However, those metabolites were not detected in our study (Figure 5.2a), probably because 1,2,3-TCB production was thermodynamically less favourable relative to the reductive removal of doubly flanked chlorine from 1,2,3,4-TeCB that leads to $1,2,4-\mathrm{TCB}$ formation (Figure 5.4).

Furthermore, 1,2,4-TCB was the only TCB detected in the 1,2,4,5-TeCB amended microcosms that was predominantly dechlorinated to $1,4-\mathrm{DCB}$ with slightly larger negative 
change of Gibbs free energy than formation of 1,3-DCB by the microcosm originated from contaminated harbor sludge (Figure 5.4). In contrast, more 1,3-DCB than 1,4-DCB was produced via 1,2,4-TCB in the transferred cultures derived from Kanaal Ieper-Ijzer sludge (Figure 5.3b\&c), indicating the impact of inoculum source on the preference for the prevailing biotransformation pathway. The formation of 1,2-DCB from 1,2,4-TCB with the removal of unflanked meta-chlorine and lowest energy release was neither observed in our microcosms (Figure 5.4) nor in other reported enrichment cultures [330, 336, 340, 354] or in studies using CB dechlorinating isolates [27, 57, 159, 335].

In our study, Dehalococcoides and Dehalobacter were detected in all TeCB-fed microcosms as putative TeCB dechlorinators. In most of the microcosms, the growth of Dehalococcoides during 161 days of cultivation was associated with the addition of new spikes of the different TeCB substrates and subsequent $\mathrm{TeCB}$ dechlorination (Figure 5.2 and 6). However, no Dehalococcoides isolates have been previously reported to dechlorinate $1,2,3,5$-TeCB to $1,4-$ $\mathrm{DCB}$ via $1,2,4-\mathrm{TCB}$ or to dechlorinate any DCBs to MCB. Hence, the detected 1,2,4-TCB and 1,4-DCB in one of the 1,2,3,5-TeCB-fed microcosm among triplicates and the MCB increase in all the 1,2,3,4- and 1,2,3,5-TeCB-fed microcosms were possibly due to the dechlorination activity of Dehalobacter or other unknown dechlorinators. Besides, Dehalococcoides and Dehalobacter both use hydrogen as the sole electron donor for reductive dehalogenation and are corrinoid auxotroph [40, 52, 57, 65]. Lack of a negative effect of vitamin B12 starvation on the 1,2,4,5-TeCB dechlorination capability of the transferred cultures indicated the presence of corrinoid producers in the microbial community that fulfill nutritional requirements of the TeCB dechlorinators. Additionally, Dehalococcoides are Gram-negative [40] and members of Dehalobacter were found resistant to vancomycin before [62], which might explain that vancomycin treatment did not fully abolish dechlorination activity. Nevertheless, strong inhibition of vancomycin on dechlorination activity was observed in our study, and the 1,2,4,5-TeCB dechlorination pathway was changed in transferred cultures (Figure S5.1). This sensitivity to vancomycin was observed before in HCB and 1,3,5-TCB fed microcosms derived from anoxic sediments [355], and therefore indicated the (in)direct involvement of vancomycin-sensitive bacteria in $1,2,4,5-\mathrm{TeCB}$ dechlorination in transferred cultures.

Bacteria belonging to the genera Desulfitobacterium and Geobacter were also detected in the TeCB-fed microcosms by qPCR (Figure 5.6). Although high abundance of Geobacter was previously detected by qPCR in HCB dechlorinating consortia [336], and Desulfitobacterium 
predominated CBs-dechlorinating bacterial communities [340], these two genera have not been previously reported to dechlorinate $\mathrm{CB}$, and hence no conclusion can be drawn on their (direct) role in dechlorination of $\mathrm{TeCB}$ isomers and corresponding daughter products. Moreover, Desulfitobacterium and Geobacter are known as facultative OHRB with versatile metabolism $[68,124]$ that can use lactate as electron donor and other electron acceptors like nitrate and sulfite supplied as media composition in our study for their growth and maintain their populations. Additionally, Desulfitobacterium spp. were reported to be capable of de novo corrinoid biosynthesis [298], and many essential genes that are involved in cobalamin synthesis and possible corrinoid transporters were found in the genome of Geobacter lovleyi [128], suggesting that these organisms may serve as corrinoid producers in the enrichments studied here.

In our study, members of the phylum Fibrobacteres were detected in all TeCB-fed microcosms, except one of three 1,2,3,5-TeCB-fed microcosms, with relative abundances ranging between 0 and $49.5 \%$ (Figure 5.5). Members of this phylum are known as plant polymer-degrading bacteria and were reported before from anaerobic cellulose-fed digesters and herbivore guts [356]. Even though no evidence has been shown for their (direct) involvement in reductive dechlorination of organohalide pollutants, they were detected before in contaminated sites like a hexahydro-1,3,5-trinitro-1.3.5-triazine (RDX) contaminted vadose zone [357, 358] and soils contaminated with cadmium [359] and crude oil [360]. High relative abundance of sulfate-reducing Deltaproteobacteria, mainly from the Desulfobacteraceae family, with relative abundances ranging between 5 and $25.9 \%$ was detected in all TeCB-fed microcosms during 161 days of cultivation (Figure 5.5a). Sulfate reducing members of the Deltaproteobacteria were suggested to support Dehalococcoides by providing essential corrinoids [237] and therefore may have a stimulatory effect on dechlorination [352]. Besides, syntrophic association between OHRB and co-existing, non-dechlorinating microbes (i.e. sulfate-reducing bacteria) was descovered before via providing substrate to carbon monoxidemetabolizing anaerobes [37].

Increase of Bacteroidetes abundance was reported before after in situ bioaugmentation to treat trichloroethene (TCE)-contaminated groudwater, which indicated a potential syntrophy and/or synergistic relationship between Bacteroidetes and chlorinated ethene dechlorinators [361]. Bacteroidetes were also found highly enriched in our study, especially from the genus Petrimonas and the class Sphingobacteriia. Members of the genus Petrimonas are known as anaerobic fermentative bacteria and were previously isolated from a biodegraded oil reservoir 
and confirmed as lactate fermentator [362]. Furthermore, Petrimonas was found predominant in a laboratory upflow anaerobic sludge blanket (UASB) reactor treating TCE containing wastewater supplied with glucose and lactate as substates, and Petrimonas showed a positive correlation with TCE removal efficiency [363]. In our study, Petrimonas was mainly detected in the transferred cultures derived from river sludge collected in Kanaal Ieper-Ijzer (Table S5.2), but not from transfers derived from the harbor sludge, in which Sphingobacteriia dominated the bacterial community (Table S5.2). Sphingobacteriia were reported before to use lactate as carbon source [364], which might explain their considerable growth found in our study. Bacterial clones affiliated with Sphingobacteriia were detected before in a TCEdegrading community after in situ lactate biostimulation [365] and sequences of Sphingobacteriia (in family WCHB1-69) were detected in a dioxin-dehalogenating enrichment derived from contaminated freashwater sediment with acetate as carbon source [366] and in RDX-contaminated groundwater after acetate biostimulation [367]. In our study, a strong increase of the Bacteroidetes population in the transferred cultures suggested a potential link (e.g. syntrophy and/or synergy) between lactate-utilizing Bacteroidetes and CBs dechlorinators. Additionally, Firmicutes, all from the Clostridiales order, were detected in the transferred cultures especially in the first transfer (Figure 5.5b). In addition to known organohalide-respiring Clostridiales belonging to the genera Dehalobacter and Desulfitobacterium, other Clostridiales were reported at high relative abundances in microcosms enriched with organohalides such as CBs [340], chlorinated ethane [368], chlorinated ethene [369] and chlorinated phenols [370] as electron acceptor and lactate as the electron donor, which suggested their role as important co-existing microbial guilds in organohalide-contaminated environments and a potential partnership between nondechlorinating corrinoid-producing Clostridiales and CBs dechlorinators [244]. One of the genera within the Clostridiales order found in this study, Sedimentibacter, was previously reported in co-culture with Dehalobacter sp. E1 and was postulated to support $\beta$ hexachlorocyclohexane $(\mathrm{HCH})$ dechlorination by providing essential co-factors (e.g. corrinoid) $[63,66]$. Sedimentibacter alone was not able to survive in $\mathrm{HCH}$-fed media [63], therefore, OHRB as detoxificator of chlorinated contaminants may have stimulating impact on the growth of other anaerobic community members.

In summary, in this study we report the reductive dechlorination of three isomers of TeCB in anoxic microcosms enriched from contaminated harbor. Thermodynamically favorable dechlorinating steps were found to be selectively followed by the TeCB-fed microcosms. 
Additionally, we confirmed the important roles of Dehalobacter and Dehalococcoides in CB dechlorination. Bacterial community profiling showed diverse non-dechlorinating bacteria, some of which may have stimulated TeCB dechlorination by providing essential electron donor and cofactors. Overall, these results provide further insight into the potential CBs dechlorination in contaminated harbor environments and the interaction between $\mathrm{TeCB}$ dechlorinators and co-existing non-dechlorinators. 


\section{Acknowledgements}

We thank Shangwei Zhang and the members of the lab of Dr. Lorenz Adrian in UFZ, Germany for technical assistance in CBs measurement, and acknowledge the China Scholarship Council for the support to Yue Lu. This work was furthermore supported by a grant of BE-Basic-FES funds from the Dutch Ministry of Economic Affairs. 


\section{Supplementary materials}
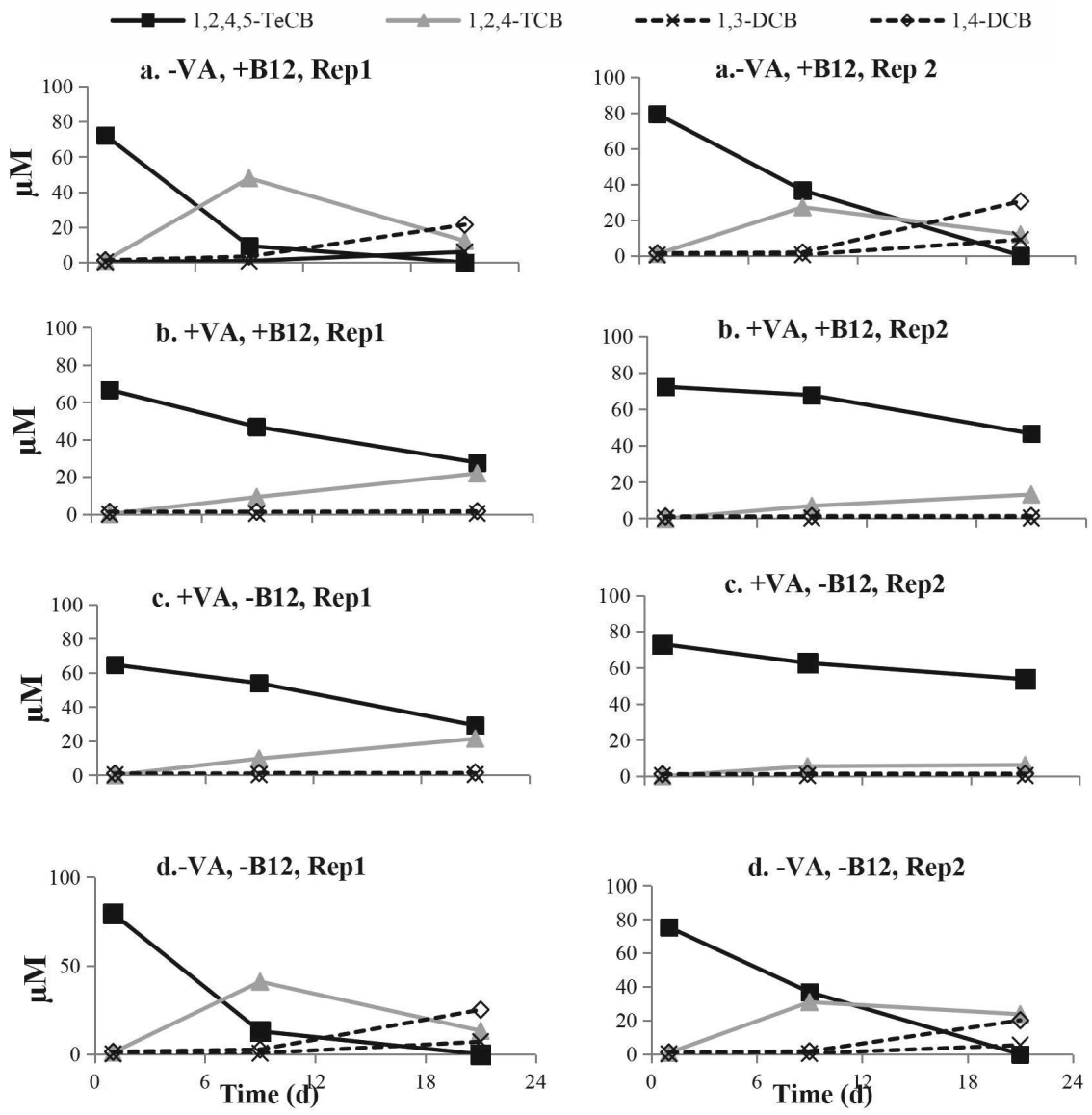

Figure S5.1 The effects of vancomycin addition and/or vitamin B12 starvation on the dechlorination activity of sediment-free 1,2,4,5-TeCB-enriched cultures derived from harbor sludge. Rep1 and 2 are replicates. +/represents addition/deficiency of certain component in the medium. 


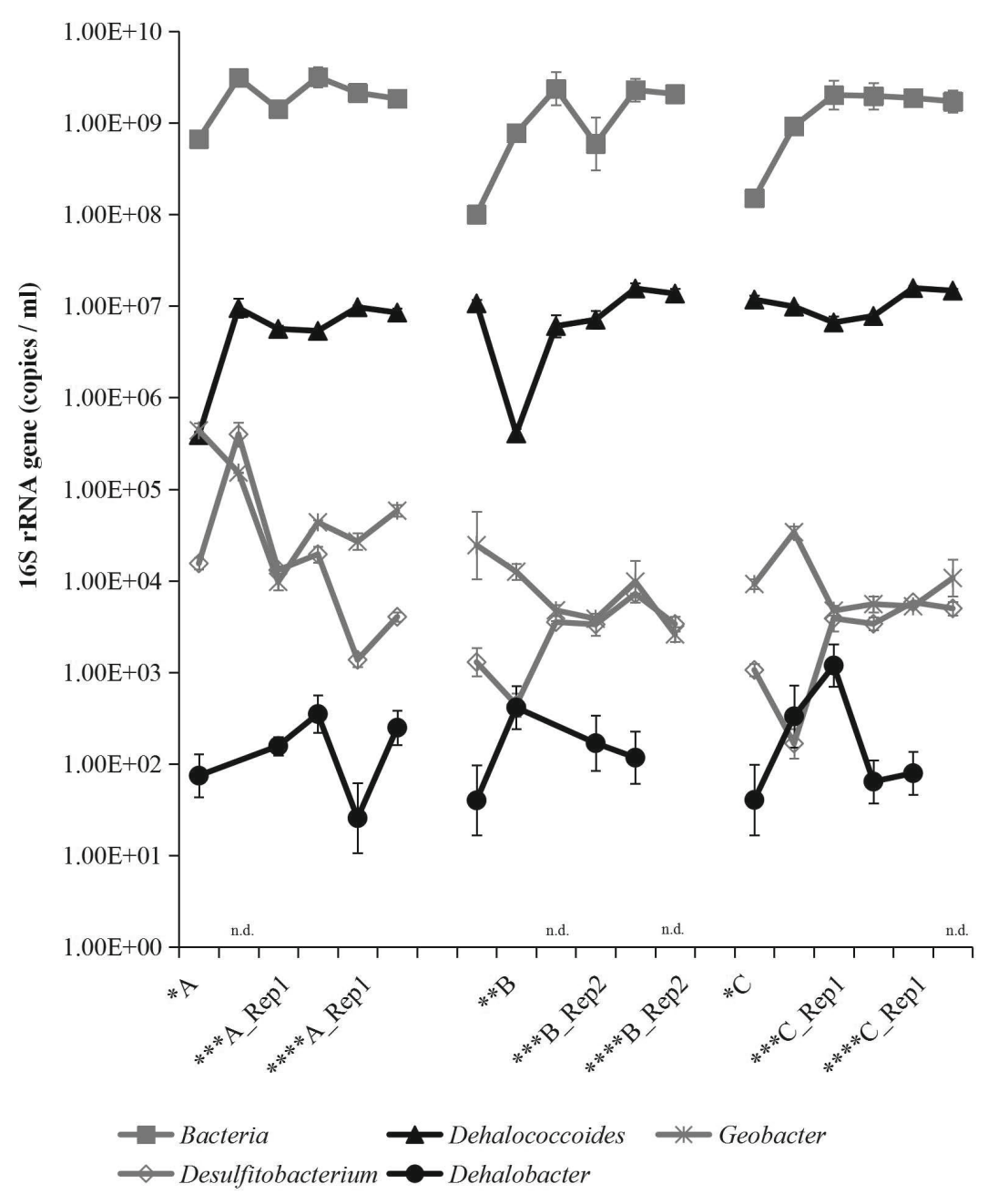

Figure S5.2 Quantitative PCR (qPCR) targeting 16S rRNA genes of Dehalobacter, Desulfitobacterium, Geobacter, Dehalococcoides and total Bacteria during serial transfers of 1,2,4,5-TeCB-enriched cultures. A, is the 1,2,4,5-TeCB-enriched culture derived from harbor sludge; B and C are 1,2,4,5-TeCB-enriched sedimentfree cultures derived from river sludge. Symbols for sampling times are: Inoculum for transfer $(*)$, First transfer_26d $(* *)$, Second transfer_66d $(* * *)$ and Second transfer_94d $(* * * *)$. Inoculum and sampling time are indicated as in Figure 5.1 (step 6). Rep1 and 2 are duplicates. Error bar is the standard deviation of technical triplicates of qPCR. n.d.: nondetectable. 
Table S5.1 Overview of the sequences of the MiSeq primers and Unitags.

\begin{tabular}{|l|l|l|}
\hline Primer $^{\mathrm{a}}$ & Oligonucleotide sequence $\left(5^{\prime}-3^{\prime}\right)^{\mathrm{b}}$ & Reference \\
\hline 27F-DegS & GTTYGATYMTGGCTCAG & {$[371]$} \\
\hline 338R-I & GCWGCCTCCCGTAGGAGT & {$[372]$} \\
\hline 338R-II & GCWGCCACCCGTAGGTGT & {$[372]$} \\
\hline Unitag1 & GAGCCGTAGCCAGTCTGC & {$[373]$} \\
\hline Unitag2 & GCCGTGACCGTGACATCG & {$[373]$} \\
\hline
\end{tabular}

a Primer names may not correspond to original publication

${ }^{\mathrm{b}} \mathrm{M}=\mathrm{A}$ or $\mathrm{C} ; \mathrm{R}=\mathrm{A}$ or $\mathrm{G} ; \mathrm{W}=\mathrm{A}$ or $\mathrm{T} ; \mathrm{Y}=\mathrm{C}$ or $\mathrm{T}$ 


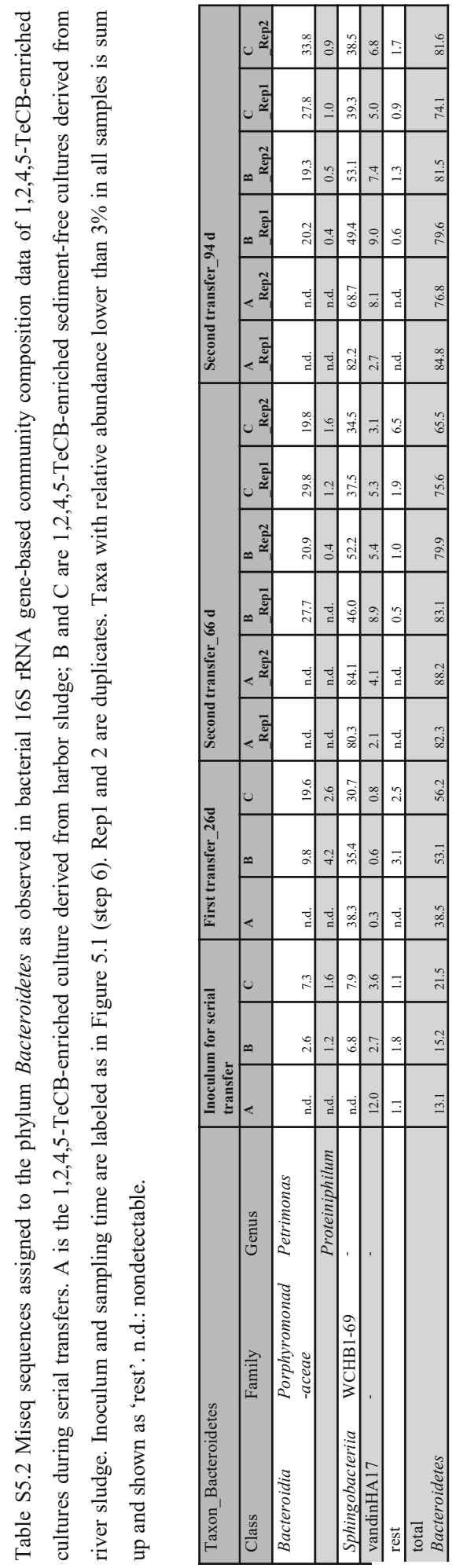


苛 


\section{Geochemical and microbial}

\section{analysis of groundwater at a site}

\section{with biostimulated reductive}

\section{dechlorination}

This chapter has been submitted for publication: Siavash Atashgahi, Yue Lu, Ying Zheng, Edoardo Saccenti, Maria Suarez-Diaz, Javier Ramiro Garcia, Heinrich Eisenmann, Martin Elsner, Alfons JM Stams, Dirk Springael,

Winnie Dejonghe, Hauke Smidt. Geochemical and microbial analysis of groundwater at a site with biostimulated reductive dechlorination. Under review. 


\begin{abstract}
Biostimulation is widely used to enhance reductive dechlorination of chlorinated ethenes in contaminated aquifers. However, the knowledge on corresponding biogeochemical responses is limited which hampers designing effective bioremediation strategies. In this study glycerol was injected in an aquifer contaminated with cis-dichloroethene (cDCE) and geochemical and microbial shifts were followed for 265 days. Highly reducing conditions prevailed in impacted wells after biostimulation. Sulfate reduction was the predominant electron-accepting process. MiSeq 16S rRNA gene sequencing revealed temporarily increased relative abundance of Firmicutes, Bacteriodetes and sulfate reducing Deltaproteobacteria with positive correlation to organic rich and reducing conditions. Dechlorination was observed towards the end of the field experiment with concomitant enrichment of ${ }^{13} \mathrm{C} \mathrm{cDCE}$ and increased Dehalococcoides mccartyi $(\mathrm{Dcm})$ numbers. However, with fading organic-rich conditions towards the end of the experiment, dechlorination stalled mostly at vinyl chloride. This was concurrent with i) reduced relative abundances of fermenting and sulfate reducing bacteria that have been suggested to support $D c m$ growth by providing electron donor and key cofactors, ii) increased relative abundance of Epsilonproteobacteria and Deferribacteres as putative oxidizers of reduced sulfur compounds but not previously described as supporters of Dcm growth. Our results imply the importance of syntrophic interactions to sustain robust dechlorination after biostimulation.
\end{abstract}




\section{Introduction}

The widespread use of chlorinated ethenes (CEs) as solvents has resulted in severe groundwater contamination [181]. The incomplete transformation of CEs such as tetrachloroethene (PCE) and trichloroethene (TCE) in aquifers leads to the accumulation of cis-dichloroethene (cDCE) and vinyl chloride (VC) [374], the latter known as a human carcinogen [375]. In situ bioremediation via enhanced reductive dechlorination (ERD) has become a widely applied remediation approach [376], and is achieved by biostimulation and in some cases bioaugmentation to activate microbial reductive dechlorination by organohalide-respiration (OHR) [188, 189, 197, 377]. Organohalide-respiring bacteria (OHRB) conserve energy by OHR [7] and belong to distinct bacterial genera distributed among the phyla Chloroflexi, Firmicutes, and Proteobacteria [378]. While many OHRB can only dechlorinate PCE and TCE to cDCE, Dehalococcoides mccartyi (Dcm) is capable of dechlorinating CEs to VC and nontoxic ethene. Dcm has been recognized as a unique key to a broad range of organohalides chemically locked for mineralization. This is due to its highly specialized metabolism restricted to $\mathrm{OHR}$, rich repertoire of reductive dehalogenase genes ( $r d h A B)$ ranging from 10 to 36 copies in a single genome [40] and widespread distribution in pristine and contaminated sites [90]. Dcm growth and dechlorination is more robust in mixed microbial communities as it is a fastidious microbe and depends on other organisms to provide hydrogen and acetate as electron donor and carbon source, respectively [40], to produce organic cofactors [320], to scavenge oxygen [237] and to remove the carbon monoxide that it produces but cannot tolerate [37].

Following addition of an electron donor in contaminated aquifers, OHRB will depend on composition and activity of the resident microbial community, the local geochemical and hydrological conditions and the interactions between these factors. Aquifers are biogeochemically and hydrologically highly heterogeneous/diverse and understanding biostimulation-induced subsurface feedback demands insight in microbial community dynamics and physiology and their interactions with geochemical and hydrological parameters across space and time. This knowledge is crucial to guide design and optimization of ERD efforts but remains limited. To date, ERD related microbial monitoring efforts have mainly focused on quantitative PCR (qPCR) based tracking of key OHRB and their $r d h A$ genes [197, 379-382] or occasionally of selected non-dechlorinating microbial guilds [361]. While application of qPCR as a specific assay has highly refined diagnostic power and guided bioremediation efforts, it normally assays a limited number of targets and hence provides only 
fragmented information regarding microbial community composition, interactions between community members and community dynamics in response to ERD. Former studies on biogeochemical trajectories of ERD either applied low resolution microbial community analysis methods such as clone libraries and terminal restriction fragment length polymorphism of PCR-amplified 16S rRNA genes [189, 365, 383] or were not complemented with detailed analysis of geochemical parameters and CE concentration dynamics [384]. Some studies have addressed microbial community dynamics $[385,386]$ and interactions with geochemical parameters [387-391] at CE contaminated sites undergoing natural attenuation without ERD. However, unlike the steady-state conditions under natural attenuation, biostimulation induces perturbations in the aquifer ecosystem that can reshape both living systems and the abiotic environment [392].

In this study an in situ glycerol injection experiment was conducted to stimulate reductive dechlorination of $\mathrm{cDCE}$ and $\mathrm{VC}$ as products of a former TCE contamination at a site in an industrial area in Vilvoorde, Belgium [199]. Before and after injection groundwater geochemical parameters and bacterial community composition were analyzed over a period of 265 days. In parallel, the progress of ERD was followed by enumeration of $\mathrm{Dcm}$ and relevant $r d h A$ genes using qPCR and monitoring of $\mathrm{cDCE}$ and VC isotopic signatures using compound specific isotope analysis (CSIA). This way, we aimed to gain an integrated geochemical and microbial insight that can be used to guide the design and optimization of ERD. Results showed how temporal succession of the predominant microbial guilds governed by their known/predicted physiology and aquifer geochemical feedback promoted and/or precluded ERD. Data presented here support the notion that microbial guilds known to sustain robust Dcm growth/dechlorination by syntrophic interactions seem pivotal for the success of ERD.

\section{Materials and methods}

\section{Site description, treatment and sampling procedures}

At the contaminated site, a $1.4 \mathrm{~km}$-wide groundwater plume mainly contaminated with cDCE flows towards the Zenne River in a northwesterly direction (Figure S6.1). Four injection wells were used to inject a single pulse of a glycerol:water mixture $(1: 3 \mathrm{v} / \mathrm{v})$ by sonic drilling injection, at $20 \mathrm{~L}$ per vertical meter from 7 to 13 meters below surface (mbs) at each injection point. Eight monitoring wells were used for groundwater sampling and monitoring. Seven wells were located downstream of the injection points (wells 1-7) and one upstream (well 0 , designated as the control well). All monitoring wells contained a shallow filter at 7-9 mbs (A- 
filters) and a deep filter at 11-13 mbs (B-filters), except the control well that only contained a shallow filter. Groundwater was sampled from the control well and the wells impacted by glycerol injection (wells 2, 3, 5, 7A; Figure S6.1) on day 0 (March 15 ${ }^{\text {th }} 2011$ ) before glycerol injection, and 37, 72, 195 and 265 days after injection as outlined in Supplementary materials.

\section{DNA extraction and qPCR}

For DNA extraction, $2 \mathrm{~L}$ groundwater samples were vacuum-filtered over a $0.22 \mu \mathrm{m}$ membrane filter (Millipore, USA), and filters were stored at $-80{ }^{\circ} \mathrm{C}$. Filters were cut into small strips for DNA extraction by the FastDNA Spin Kit for Soil (MP Biomedicals). qPCRs were performed in triplicate in $25-\mu \mathrm{L}$ reactions in an iQ5 iCycler using the iQ SYBR Green Supermix kit (Bio-Rad, Veenendaal, the Netherlands). A list of target genes, primers and thermal cycling conditions for qPCRs is shown in Table S6.1. Standard curves were obtained using serial dilutions of a known concentration of plasmid DNA containing a suitable fragment of the target genes.

\section{Bacterial community analysis}

A 2-step PCR strategy was used to generate barcoded amplicons from the V1-V2 region of the 16S rRNA gene (primers and thermal cycling conditions shown in Table S6.2) as shown in chapter 5. The PCR product was purified with HighPrep ${ }^{\mathrm{TM}}$ (Magbio Genomics, Rockville, MD, USA) and quantified using a Qubit 2.0 Fluorometer (Life Technologies, Darmstadt, Germany). Purified PCR products were pooled and sequenced on a MiSeq platform (GATCBiotech, Konstanz, Germany).

\section{Analysis of the MiSeq data}

NG-Tax, an in-house pipeline [346] was used for the analysis of the 16S rRNA gene sequencing data. In brief, paired-end libraries were filtered to contain only read pairs with perfectly matching barcodes, and those barcodes were used to separate reads by sample. Finally operational taxonomic units (OTUs) were assigned using an open reference approach and a customized SILVA 16S rRNA gene reference database [348]. Bacterial composition plots were generated using a workflow based on Quantitative Insights Into Microbial Ecology (QIIME) v1.2 [349].

\section{Chemical and carbon isotope analysis:}

Concentrations of CEs, ethene, ethane, and methane in groundwater samples were determined on a Varian GC-FID (CP-3800) as described previously [226]. Sulfate concentrations were analyzed by ion chromatography using a Dionex DX-120 ion chromatograph equipped with a 
Dionex AS14A column (Dionex, Sunnyvale, CA). Concentrations of aqueous Fe(II), total Fe and $\mathrm{NO}_{3}-\mathrm{N}$ were determined using $\mathrm{HACH}$ kits (HACH, USA) according the manufacturer's instructions. Dissolved organic carbon (DOC) was determined from samples as the difference between total dissolved carbon and dissolved inorganic carbon, measured with a Shimadzu TOC-5000 analyser equipped with an ASI-5000 auto-sampler. The stable carbon isotope composition of CEs in the groundwater samples were determined as described in Supplementary materials. Concentration-weighted average values of the isotope signature for CEs ( $\Sigma\left(\right.$ EC) was calculated by weighting compound-specific values $\delta^{13} \mathrm{C}$ weighted by their molar fractions (x) [393]:

$$
\delta^{13} C_{\Sigma(C E)}=\delta^{13} C_{D C E} x_{D C E}+\delta^{13} C_{V C} x_{V C}
$$

\section{Statistical analysis}

In order to compare microbial communities observed at different times and locations, different statistical approaches were used based on Bray-Curtis distances. PERMANOVA (Permutational Multivariate Analysis of Variance) [394] and ANOSIM (Analysis of Similarity) [395] were performed using the Fathom Toolbox for Matlab [396]. We used $10^{4}$ permutations to assess significance of observed differences. MixOmics R package [397, 398] was used to integrate microbiota abundance data and geochemical parameters and to perform regression analysis. Sparse partial least squares was used for simultaneous variable selection and integration to avoid selection of spurious associations. Geochemical (microbiota) data were set as dependent (independent) variables. Interchanging both sets did not significantly impact the results. An additional threshold on correlation values was imposed by retaining only the $20 \%$ most relevant associations in the final network.

\section{Nucleotide sequences}

Nucleotide sequence data reported are available at the European Bioinformatics Institute under accession number PRJEB13312.

\section{Results}

\section{Geochemical characterisation}

Geochemical parameters were stable in the control well throughout the experiment. After biostimulation, DOC in the impacted filters was significantly $(P<0.05)$ higher than before biostimulation over the experiment duration (Figure S6.2, Table S6.3). The opposite pattern was observed for dissolved oxygen (DO) and oxidation-reduction potential (ORP) that declined and were significantly $(P<0.05)$ lower throughout the experiment relative to pre- 
biostimulation values. ORP and DO slightly increased towards the end of the experiment. Nitrate was not detected. Fe(II) concentrations varied between day 0 and 72 in the downgradient wells but showed a significant $(P<0.05)$ reduction by day 195 and 265 relative to prebiostimulation concentrations. This was accompanied by appearance of black precipitate indicative of ferrous sulfide formation. In most samples throughout the experiment, the $\mathrm{Fe}(\mathrm{II}) / \mathrm{Fe}$ ratio was close to 1 indicating lack of reducible Fe(III). Starting from a background concentration of $463 \pm 133 \mathrm{mg} / \mathrm{l}$, sulfate steadily decreased to $212 \pm 116 \mathrm{mg} / \mathrm{l}$ by day $195 \mathrm{in}$ down-gradient wells and rebounded in most wells by day 265 reaching $267 \pm 180 \mathrm{mg} / \mathrm{l}$ (Figure S6.2, Table S6.3).

\section{Reductive dechlorination of CEs, CSIA and qPCR analysis}

The pre-stimulation carbon isotope values of cDCE (-19.3\% on average) and the sum of carbon isotope values of all CEs weighted by their molar fractions $\left(\delta^{13} \mathrm{C}_{\Sigma(\mathrm{CE})},-21.4 \%\right.$ on average) were highly consistent in the seven filters later impacted by biostimulation (SD < 1\%). By day 195, there was clear onset of cDCE dechlorination with concomitant increase in its $\delta^{13} \mathrm{C}$ values and increased VC concentration in most filters. $\delta^{13} \mathrm{C}$ of VC remained more negative compared to $\mathrm{CDCE}$ in shallow filters of $2 \mathrm{~A}, 3 \mathrm{~A}$ and $7 \mathrm{~A}$ and $\mathrm{VC}$ was not further transformed to ethene. Further degradation of cDCE to VC was observed at day 265 with progressive enrichment of $\mathrm{cDCE}$ in ${ }^{13} \mathrm{C}$. In addition, a significant increase (3\%o) in the resultant $\delta^{13} \mathrm{C}_{\Sigma(\mathrm{CE})}$ relative to the control well provided evidence for incipient dechlorination of VC in filters 2A, 2B and 7A between day 195 and 265 (Figure 6.1, Table S6.4) [399]. The evidence from $\mathrm{CE}$ concentrations and isotopic signatures was further corroborated by over 1-2 orders of magnitude increased abundances of $D c m$ and $v c r A$ and $b v c A$ genes in the impacted wells relative to the control well. Among the $r d h$ genes, $b v c A$ became dominant in most filters, whereas $v c r A$ was dominant in well 2 reaching above $10^{7}$ copies/L by day 265 . No ERD was noted in well 5 and the control well, in which cDCE concentration as well as isotopic and qPCR signatures were rather stable (Figure 6.1, Table S6.5).

\section{Impact on bacterial community alpha-diversity}

Biostimulation significantly affected bacterial community alpha-diversity. The postbiostimulation samples (seven samples at each sampling time point, excluding control well samples) showed significantly higher $(P<0.05)$ predicted (Chao 1$)$ and observed OTU 

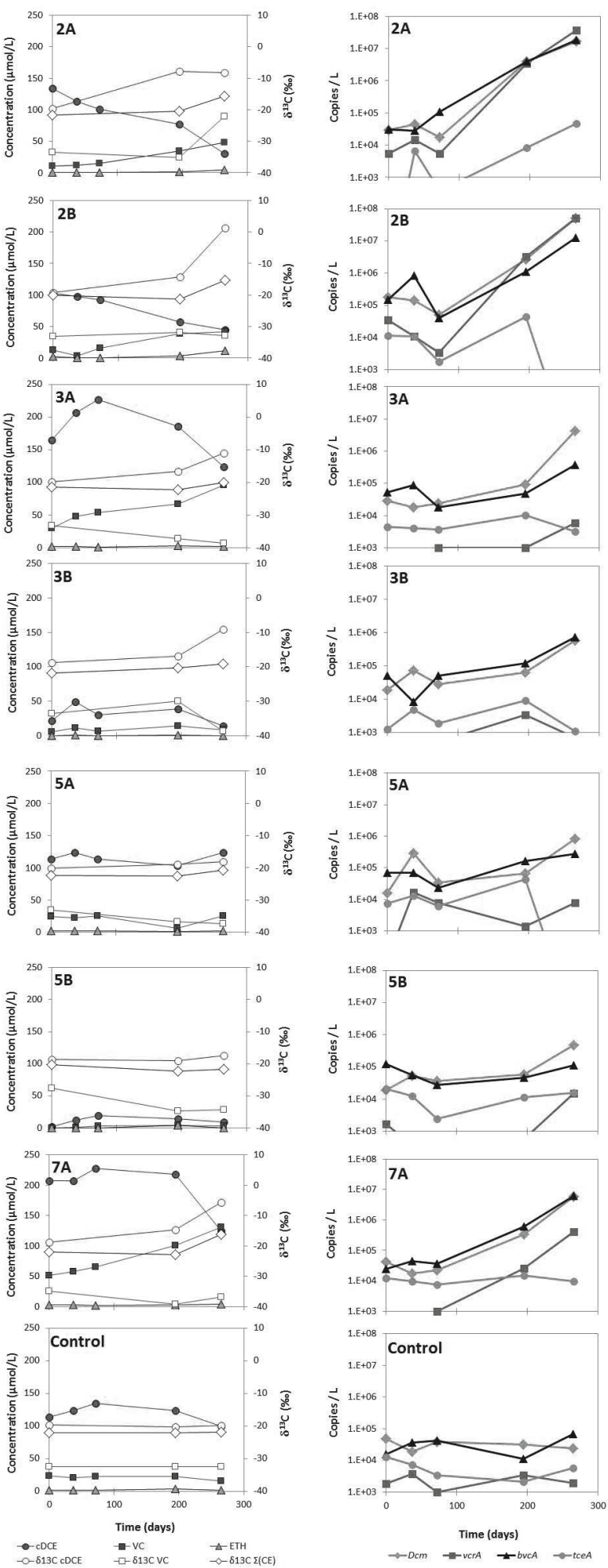
Figure 6.1 Evolution of reductive dechlorination over time. Left: concentrations of cDCE, VC, ethene, $\delta^{13} \mathrm{C}$ of $\mathrm{cDCE}$ and $\mathrm{VC}$ and concentration-weighted average of $\mathrm{CE} \delta^{13} \mathrm{C}$ values $\left(\delta^{13} \mathrm{C} \Sigma(\mathrm{CE})\right)$. Right: qPCR quantification of 16S rRNA gene copy numbers of $D c m$ and reductive dehalogenase encoding $v c r A, b v c A$ and tceA genes. Each qPCR value represents the average of triplicate reactions. ETH: ethene.
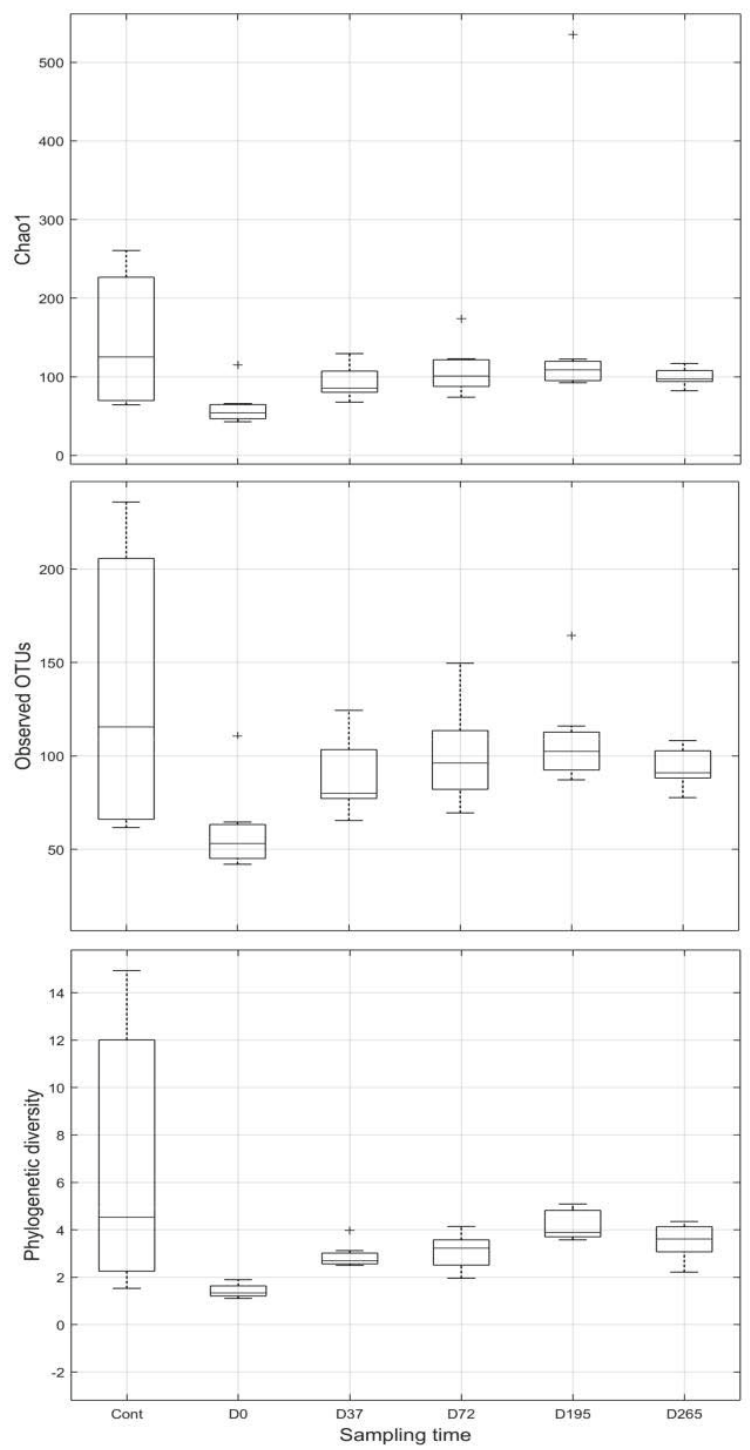

Figure 6.2 Predicted (Chao 1) and observed OTU richness, and phylogenetic diversity in the control (five samples) and impacted filters at each sampling campaign (seven samples). Cont: control well. 
richness, and phylogenetic diversity (PD) relative to the pre-biostimulation samples (Figure 6.2). No significant difference was found between the post-biostimulation sampling dates, except for the PD at day 195 that was significantly higher $(P<0.05)$ than at day 37 and 72 . The control well showed progressively increasing alpha-diversity over time until day 195 (Figure S6.3).

\section{Bacterial community succession}

The pre-biostimulation bacterial community composition showed high between-sample similarity including the control samples (Figure S6.4), and was significantly different from all post-biostimulation communities (Table S6.7). Remarkably, among the post-biostimulation communities, samples of day 265 were not significantly different from those taken at the well (Figure S6.4, Table S6.6). At day 0, the bacterial community was dominated by Campylobacterales (Epsilonproteobacteria) followed by Flavobacteriales (Bacteroidetes), representing $75 \%$ and $18 \%$ of the average relative abundance (ARA), respectively (Figure 6.3, Table S6.6). Recorded Campylobacterales comprised members of Sulfuricurvum, Sulfurospirillum and Arcobacter genera, while Flavobacterium was the main genus within Flavobacteriales (Figure S6.5, Table S6.6). Following biostimulation, clear shifts in the bacterial community structure were noted. Except in the $2 \mathrm{~B}$ and $7 \mathrm{~A}$ filters that did not show DOC change at day 37 (Figure S6.2, Table S6.4), Campylobacterales and Flavobacteriales were greatly reduced in ARA relative to day 0 and replaced by fermentative members of the Firmicutes (Clostridia and Bacilli orders) and Bacteroidetes (Bacteroidia order) and by sulfate reducing Deltaproteobacteria (Desulfobacterales order) (Figure 6.3, Table S6.6). Within the Campylobacterales members of the genus Sulfurospirillum became predominant whereas Sulfuricurvum, Arcobacter and Flavobacterium showed drastic reduction in ARA. Members of the genus Trichococcus (Bacilli) within the Firmicutes increased substantially in relative abundance in 5A and 5B filters (Figure S6.5, Table S6.6) that received the highest DOC input, whereas Clostridia genera of Acetobacterium, Clostridium and Pelosinus were noted in most filters. By day 72, Campylobacterales and Flavobacteriales dropped to below 20 and $1 \%$ ARA, respectively in impacted filters. In contrast, the sulfate reducing Desulfobacterales dominated by Desulfobulbus genus flourished followed by Bacteroidia, Clostridia and Bacilli. Desulfobacterales continued to dominate communities observed in day 195 samples while Bacteroidia, Clostridia and Bacilli were fading. Interestingly, at day 195 and day 265, the Campylobacterales became predominant again in 5A and 5B filters, reaching $60 \%$ and $30 \%$ in relative abundance, respectively. Instead, Desulfobacterales were reduced in 


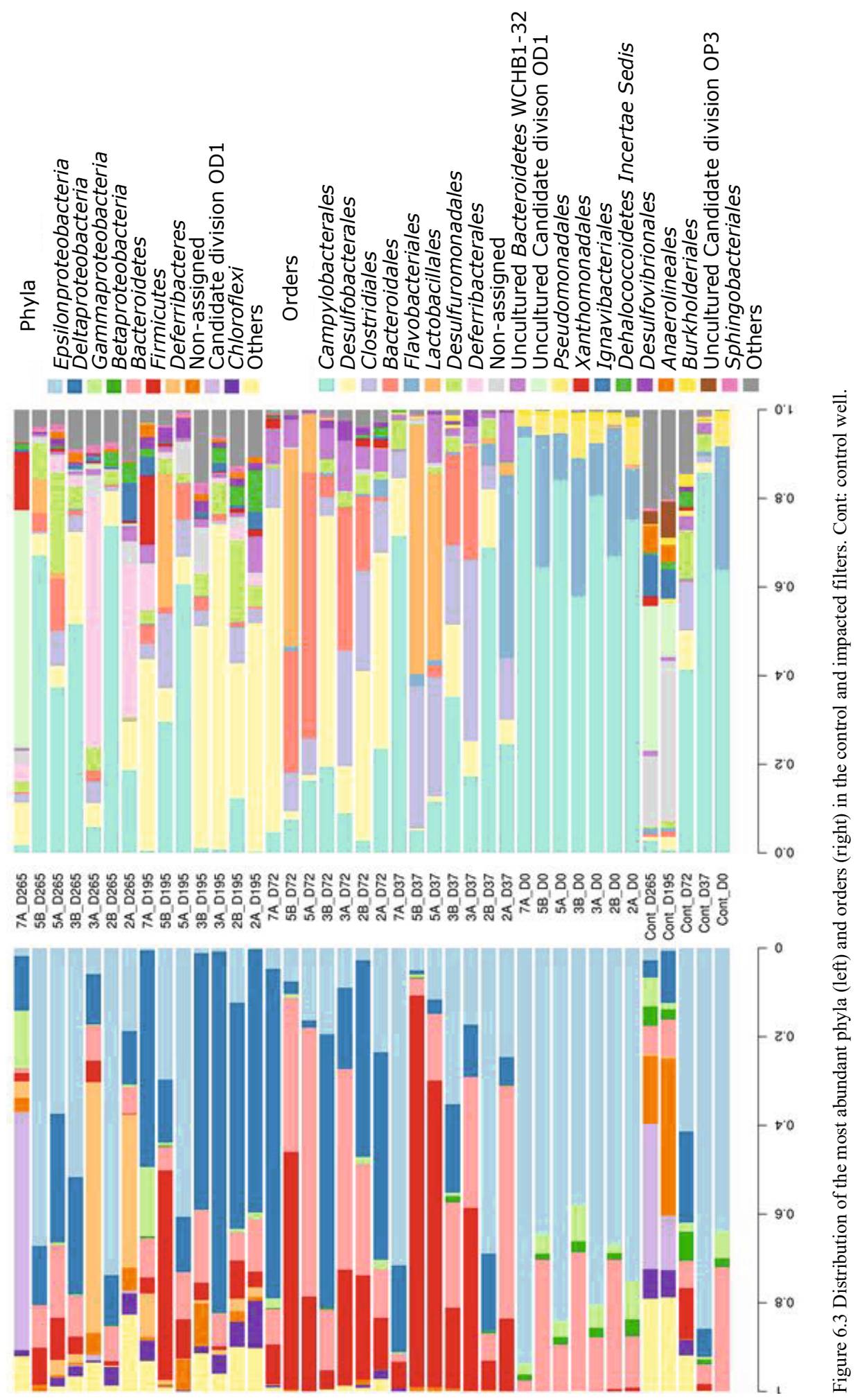




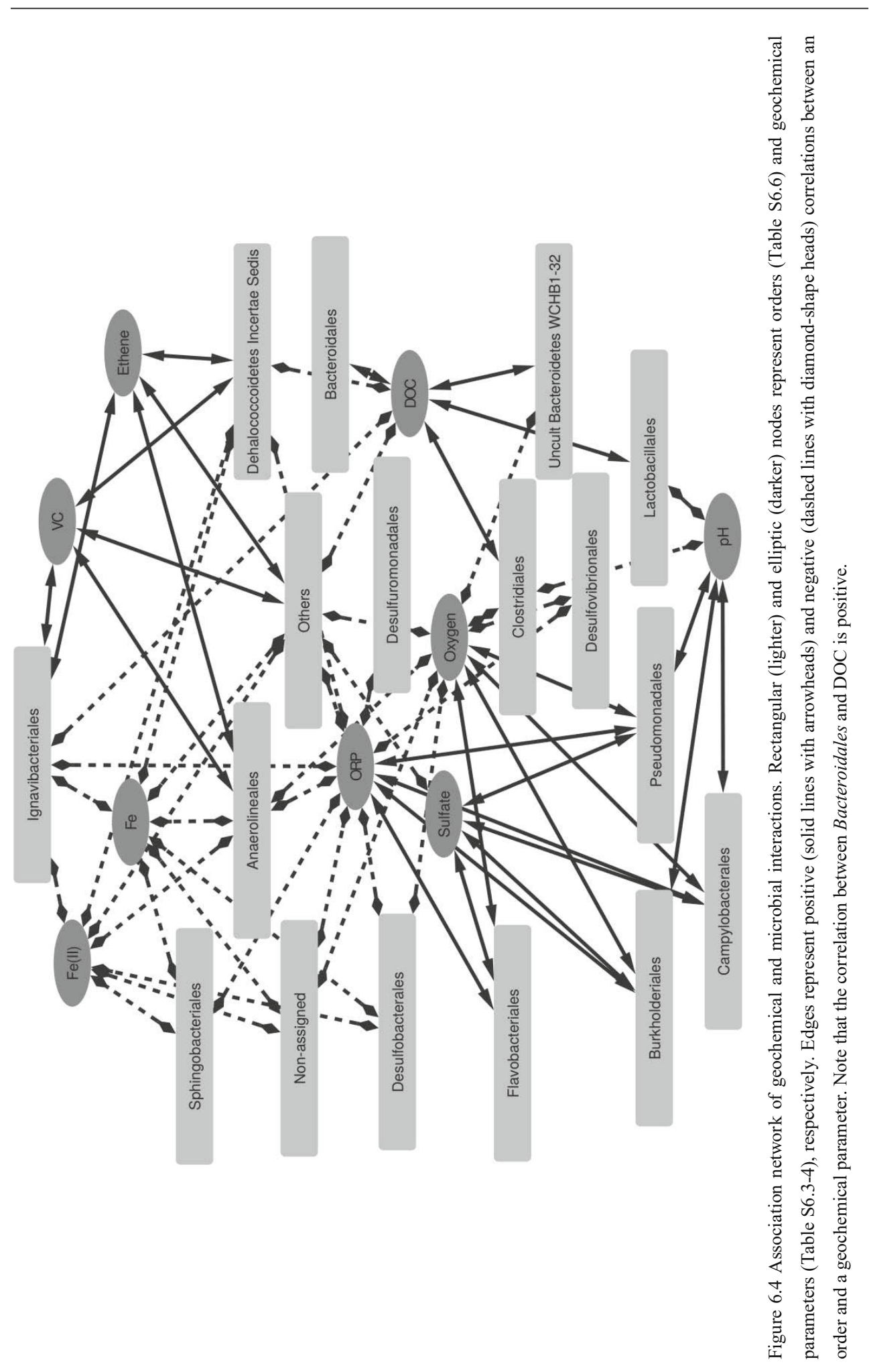


ARA at day 265. Dehalococcoidetes and Geobacter did not surpass 1\% of the community at any time point, and other OHRB including Dehalobacter, Desulfitobacterium, Desulfomonile, Desulfuromonas were not detected. Furthermore, there was notable emergence of sequences associated with Deferribacterales (Deferribacteres) and the candidate phylum OD1 by day 195 and 265, which were not observed in pre-biostimulation samples. The bacterial community showed a distinct succession pattern in the control well compared to stimulated wells. While Campylobacterales was the most predominant taxon at day 0 and 37, their relative abundance dropped to $41 \%$ by day 72 and below $2 \%$ by days 195 and 265 . The community in the control well became more diverse over time (Figure 6.2) with increased abundance of non-assigned reads and candidate phylum OD1 (Figure 6.3, Table S6.6).

\section{Geochemical and microbial interactions}

The relative abundances of the orders Campylobacterales, Flavobacteriales, Burkholderiales and Pseudomonadales were positively correlated with DO, ORP and sulfate (Figure 6.4 and S7.6). Putative fermenters belonging to Clostridiales, Lactobacillales, Bacteroidales and uncultured Bacteriodetes WCHB1-32 showed a positive correlation with DOC whereas DO was inversely correlated with the relative abundance of Clostridiales and uncultured Bacteriodetes WCHB1-32. A negative correlation was noted between $\mathrm{Fe}(\mathrm{II}) / \mathrm{Fe}$ and Dehalococcoidetes.

\section{Discussion}

There is growing interest in using the capacities of indigenous microbial communities to remediate $\mathrm{CE}$ contaminated aquifers by $\mathrm{ERD}$. In order to improve $\mathrm{CE}$ contaminated site bioremediation, the biogeochemical shifts induced by biostimulation must be understood, and the abundance and activity of key OHRB must be placed into the context of interlinked microbial networks, including the supporting/competing electron donor/acceptor processes.

\section{Geochemical and microbial dynamics: pre-biostimulation phase}

The almost complete removal of TCE and the dominance of CDCE before biostimulation indicated naturally occurring reductive dechlorination and hence TCE depletion (Table S6.4) formerly reported to be present at this site [199]. This could be an explanation for the presence of Sulfurospirillum as non-obligate dechlorinator of TCE to CDCE [142] in the prebiostimulation samples that was also reported from other TCE contaminated aquifers [365, $383,400]$. Some members of this genus are capable of chemolithoautotrophic growth by coupling nitrate and oxygen reduction to the oxidation of sulfide, sulfur and thiosulfate [142, 
401] and therefore, their growth using sulfur released into groundwater from soils, rocks and minerals [402] coupled to oxygen reduction cannot be excluded. Sulfuricurvum as another predominant genus currently contains only one characterised isolate, Sulfuricurvum kujiense strain YK-1, an obligate chemolithoautotroph [403] that grows by oxidation of reduced sulfur compounds coupled to nitrate and oxygen (microaerophilic condition) respiration (Kodama and Watanabe, 2003). Although dechlorination or syntrophic interactions with OHRB by this strain are not reported, Sulfuricurvum was detected at TCE contaminated sites [404, 405] and in 1,2-dichloroethane dechlorinating enrichment cultures [368]. Another predominant genus in pre-biostimulation samples was Flavobacterium whose members play an important role in decomposition of organic materials by hydrolysing organic polymers such as proteins and polysaccharides produced from cell debris in oligotrophic environments [406, 407].

\section{Geochemical and microbial dynamics: post-biostimulation phase}

Glycerol injection induced cDCE concentration reduction accompanied by a shift of carbon isotope values. The most dynamic period was after day 72 during which the average $\delta^{13} \mathrm{C}$ cDCE values increased by $+3.9 \%$ at day 195 and by $+5.7 \%$ at day 265 (Figure 6.5 ). This development was reflected by a successional change in groundwater bacterial community composition. There was a sharp increase in Firmicutes (Clostridiales and Lactobacillales) ARA followed by Bacteroidetes (Bacteroidales). These taxa that also showed positive correlation to DOC (Figure 6.4) had low initial ARA and decreased again towards the end of the field experiment. Other studies at CE contaminated sites reported a similar transient peak of these microbes upon biostimulation [361, 365, 384]. The emergence of members of the genera Trichococcus, Clostridium and Pelosinus following biostimulation (Figure S6.5, Table S6.6) is likely due to their glycerol fermentation capacity [408-411]. Moreover, Pelosinus strains were shown to sustain growth of $D c m$ by providing the necessary corrinoids $[95,300]$. The observed putative acetogenic bacteria belonging to Acetobacterium, Clostridium and Spirochaetaceae (Figure S6.5, Table S6.6) have been proposed to stimulate Dcm growth by production of acetate and corrinoid cofactors [324, 361, 365].

Sulfate reduction appeared to be the prevalent electron-accepting process at the site. Between day 37 and 195, Desulfobulbaceae flourished that can perform incomplete oxidation of propionate coupled to sulfate reduction [412] yielding acetate and sulfide that can stimulate and inhibit Dcm growth, respectively. On the other hand, both glycerol and its degradation product 1,3-propanediol can be converted to acetate using sulfate as electron acceptor by members of the genus Desulfovibrio [413, 414] that were present during the same period. 


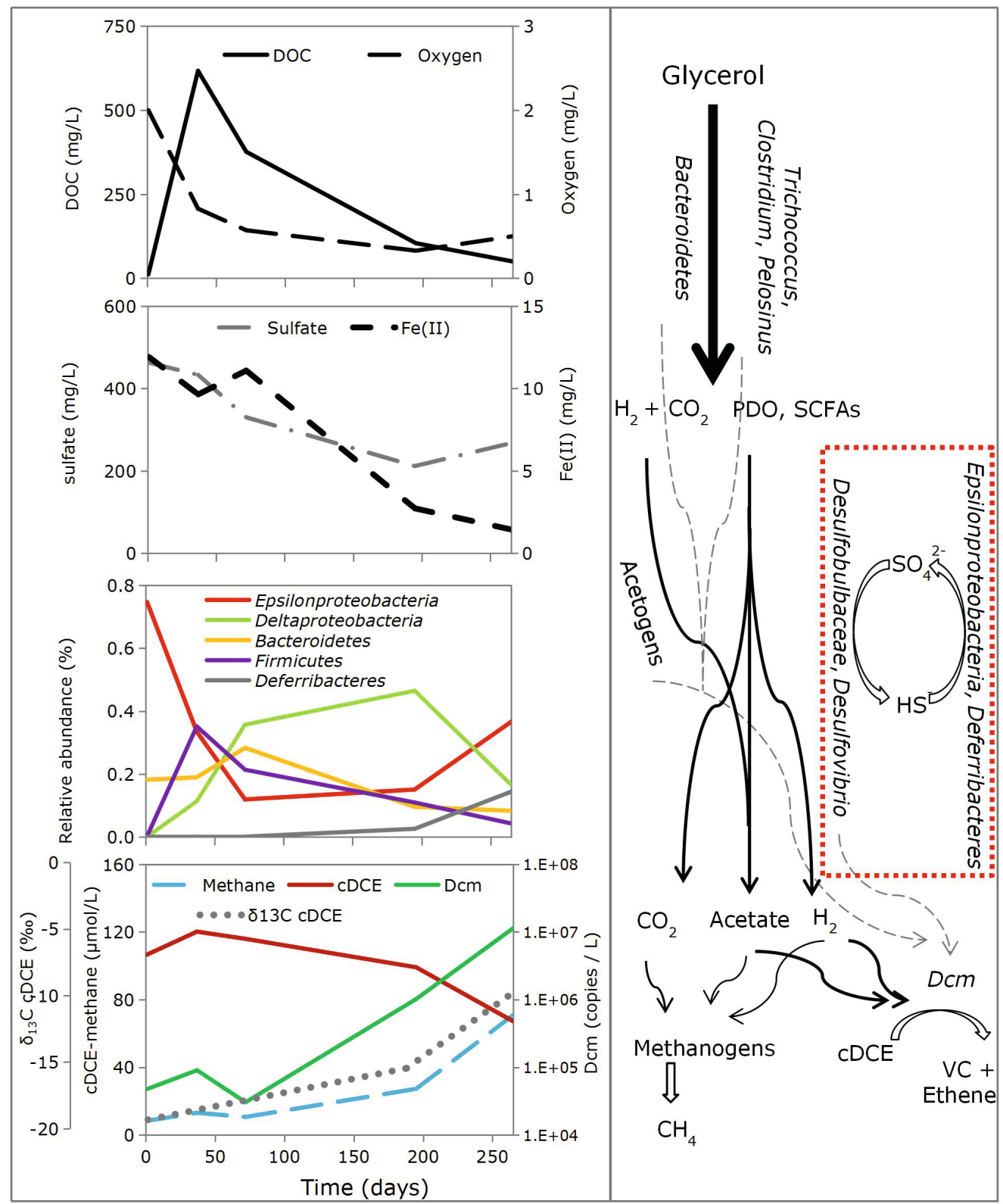

Figure 6.5 Summary of major geochemical and microbial evolution before and after biostimulation (left), and a conceptual model of glycerol degradation based of the observed taxa from MiSeq analysis and their known/putative physiology (right). The proposed sulfur cycle is shown in red dotted square and the putative corrinoid supply to $D \mathrm{~cm}$ is shown by dashed gray arrows. All values are averages of the seven impacted filters. Error bars are not shown for clarity. PDO: 1,3-propanediol, SCFA: short chain fatty acids. 
Further, Desulfovibrio strains are known to sustain $\mathrm{Dcm}$ growth by providing hydrogen, acetate and corrinoid cofactors [325]. However, sulfate at $>500 \mathrm{mg} / 1$ as observed in $3 \mathrm{~B}$ and 7A filters (Figure S6.2, Table S6.3) might be inhibitory to Dcm due to electron donor exhaustion [415], though this seems unlikely on the basis of the observed dechlorination at these filters (Figure 6.1). Methanogenesis as a sink of hydrogen and acetate [240] did not seem to be a major concern for OHR except at filters 5A and 5B that showed the highest DOC levels upon biostimulation and enhanced methanogenesis by day 265 . This can be an explanation for lack of dechlorination at these filters indicating a competitive advantage for dechlorination over methanogenesis in other filters with lower DOC levels [239].

\section{Geochemical and microbial dynamics: the control well}

Despite stable geochemical parameters in the up-gradient control well, the bacterial community observed at days 195 and 265 highly diverged from the original state. This could be due to the high hydraulic conductivity of 1-3 m/day at this site [222] facilitating a rapid dispersion and community succession [416]. Accordingly, the pre-biostimulation bacterial community in the stimulated wells was significantly different from the control well (Table S6.7) whereas the geochemical parameters were similar between the pre-biostimulation and control well samples and clustered closely in the PCA plot (Figure S6.7). On the other hand, the bacterial community in the stimulated wells at day 265 was significantly different from the pre-biostimulation community but not from the control well (Table S6.7). This indicates that the resident taxa in the impacted wells exploiting the stimulated conditions were also exposed to the arriving immigrant taxa brought by groundwater flow, in line with what has previously been suggested by Shade and co-workers [392]. However, it was only towards the end of the experiment (and hence fading stimulation) that the immigrant taxa became established in stimulated wells which can be described by the niche occupation concept outlined in Figure S6.8. The observed geochemical stability but compositional instability of the control well over time indicates the necessity of previous knowledge of community behavior in relation to site geochemistry/hydrology as baseline prior to biostimulation to better guide intensity, duration and location of post-biostimulation sampling.

\section{Putative sulfur cycle following biostimulation}

After the dominance of sulfate reducers between day 37 and 195 and concurrent sulfate depletion, sulfate concentrations increased again in most wells at day 265. This was accompanied by a substantial reduction of Desulfobulbaceae ARA, re-establishment of initially abundant Sulfuricurvum and Sulfurospirillum, and appearance of putative sulfur 
oxidizers i.e. Deferribacteres and the candidate phylum OD1, which were barely detectable in pre-biostimulation samples (Figure 6.3). The chemolithoautotrophic oxidation of the reduced sulfur compounds by Sulfuricurvum and Sulfurospirillum coupled to reduction of DO that was slightly increasing during the late phase may explain the increased sulfate levels. Accordingly, the epsilonproteobacterial Campylobacterales showed positive correlation with DO and ORP (Figure 6.4). Enrichment of Epsilonproteobacteria as the successors of Deltaproteobacteria was previously reported during biostimulation of aquifers contaminated with uranium [161, 417] and CEs [384]. The resulting reduced sulfide levels can in turn support reductive dechlorination by decreasing the toxicity of sulfide to $\operatorname{Dcm}[161,415]$.

\section{Emergence of candidate phylum OD1}

Members of the candidate phylum OD1 have mostly been connected with suboxic and anoxic pristine sulfur-rich environments [418-420]. However, OD1 [421] and another candidate division, OP11, [384] also appeared at a later stage during aquifer biostimulation for CE and uranium bioremediation possibly due to ample presence of reduced sulfur compounds (as products of sulfate reducers) and the sulfur oxidation capacity of these candidate phyla [419, 422]. Besides, owing to their obligatory fermentative lifestyle, the production of hydrogen and organic acids (e.g. acetate) from complex organic materials such as decaying biomass from microbial blooms by these candid phyla can fuel respiratory processes with nitrate, sulfate and Fe(III) [422, 423] and perhaps CEs as terminal electron acceptors. Interestingly, OD1 has not been reported as member of CE dechlorinating communities in enrichment cultures $[129,154$, 236, 237, 352, 424-429] that usually receive constant supply of easily accessible electron donors, presumably circumventing the services provided by this phylum and hence exterminating it from the dechlorinating enrichment cultures.

\section{Resilient and sensitive taxa}

Epsilonproteobacterial Sulfuricurvum and Sulfurospirillum recovered in ARA towards the end of the experiment, though remarkably only in stimulated wells. Resilience of the members of Sulfurospirillum spp. was previously reported in permanganate treated enrichment cultures [352]. In contrast, the initially predominant genera Arcobacter and Flavobacterium did not recover, which could be due to their high sensitivity to biostimulation-induced perturbation. However, they were also barely detectable from the up-gradient control well towards the end of the experiment indicating a role of other factors such as hydrology in controlling community succession. 


\section{Conclusions and perspectives}

Based on the observed geochemical, isotopic and bacterial patterns, a conceptual model of the metabolic interactions within the microbial foodweb during biostimulation was proposed (Figure 6.5). cDCE dechlorination was only noted by day 195 and it was not fully converted to ethene likely due to the fading organic-rich condition towards the end of the experiment. Remarkably, during the same period, the relative abundance of non-dechlorinating fermenters and sulfate reducers decreased that is considered to stimulate robust $\mathrm{Dcm}$ dechlorination by providing organic cofactors such as the key vitamin B12 [237, 325]. The increased relative abundance of Deferribacteres and Epsilonproteobacteria by the end of the field experiment likely supported the $\mathrm{Dcm}$ population by reducing sulfide level/toxicity. However, to our knowledge, these taxa are not known to provide the more important organic cofactors such as vitamin B12 needed by Dcm, hence they are not likely "sufficient" as companions to sustain Dcm growth. In line with this, other studies showed the importance of particular syntrophic partners belonging to Firmicutes and Deltaproteobacteria in providing the "right" cobamide lower base of vitamin B12 that is vital for Dcm dechlorination [95, 300] but cannot be provided even by closely related microbes [93, 299].

In case of incomplete dechlorination, biostimulation is performed together with bioaugmentation with dechlorinating enrichment cultures containing Dcm populations but also their non-dechlorinating partners [361, 383]. In fact, sustained presence of nondechlorinating guilds that support Dcm growth might be the key to success of bioaugmentation as opposed to the initial biostimulation. Accordingly, a recent field biostimulation failed to induce complete dechlorination while dechlorination to ethene was achieved after bioaugmentation. This was concurrent with enrichment of Bacteroidetes while Dcm and $v c r A$ concentrations were rather stable [361]. Therefore, in addition to monitoring dechlorinating guilds, future ERD monitoring efforts must consider composition and successional patterns of supportive non-dechlorination community members as well as geochemical factors controlling them to ensure robust $\mathrm{Dcm}$ growth and activity. This knowledge is pivotal to establish and maintain the required syntrophic relationships for OHR under challenging field conditions. 


\section{Acknowledgements}

This study was supported by a VITO/KU Leuven PhD scholarship (EU FP7 project AQUAREHAB, grant 226565) to Siavash Atashgahi. Furthermore, Siavash Atashgahi and Hauke Smidt received support by a grant of BE-Basic-FES funds from the Dutch Ministry of Economic Affairs and Dirk Springael by the Inter-University Attraction Pole (IUAP) " $\mu$ manager" of the Belgian Science Policy (BELSPO, P7/25). We thank Richard Lookman for his assistance in the field experiment and acknowledge the China Scholarship Council for the support to Yue Lu and Ying Zheng. 


\section{Supplementary materials}

\section{Sampling procedure}

All samples were collected using a peristaltic pump in polyethylene tubes (Eijkelkamp, Giesbeek, The Netherlands), following purging until electrical conductivity, $\mathrm{pH}$, dissolved oxygen (DO), temperature and oxidation-reduction potential (ORP) parameters stabilized. Those parameters were measured using a flow-through cell (Eijkelkamp, Giesbeek, The Netherlands), equipped with a multimeter (MultiLine F/SET3, WTW, Weilheim, Germany) and suitable electrodes for determining temperature and electrical conductivity (TetraCon 325, WTW, Weilheim, Germany), pH (Sen Tix 41, WTW, Weilheim, Germany), DO (CellOx 325, WTW, Weilheim, Germany) and ORP (Oxitrode Platinum Hamilton, Bonaduz, Switzerland). Samples for stable carbon isotope analysis were collected in $250 \mathrm{~mL}$ amber bottles without leaving headspace, and immediately treated with $\mathrm{NaOH}$ pellets to reach a $\mathrm{pH}$ above 11 to stop microbial activity.

\section{Carbon isotope analysis}

The stable carbon isotope composition of chlorinated ethenes (CEs) in the groundwater samples were determined on a gas chromatograph-combustion-isotope ratio mass spectrometer (TRACE GC Ultra, GC combustion interface, MAT 253, all from Thermo Fisher Scientific, Bremen, Germany). Each sample was analyzed in duplicate via purge-andtrap, cryofocussing and subsequent analysis on a VOCOL $60 \mathrm{~m} \times 0.25 \mathrm{~mm}$ ID. Authentic laboratory standards were used for identification of chlorinated compounds and to improve the accuracy of ${ }^{13} \mathrm{C} /{ }^{12} \mathrm{C}$ analyses by linear correction of raw isotope values. Isotope ratios (R) of samples are expressed in the delta notation $\left(\delta^{13} \mathrm{C}\right)$ in per mil [\%o] relative to the international Vienna Pee Dee Belemnite standard $\left(\mathrm{VPDB},{ }^{13} \mathrm{C} /{ }^{12} \mathrm{C}=(11237.2 \pm 2.9) \times 10^{-6}\right)$ according to:

$$
\delta^{13} C_{\text {sample }}[\% 0]=\left(\frac{R_{\text {sample }}-R_{\text {standard }}}{R_{\text {standard }}}\right) \times 1000
$$




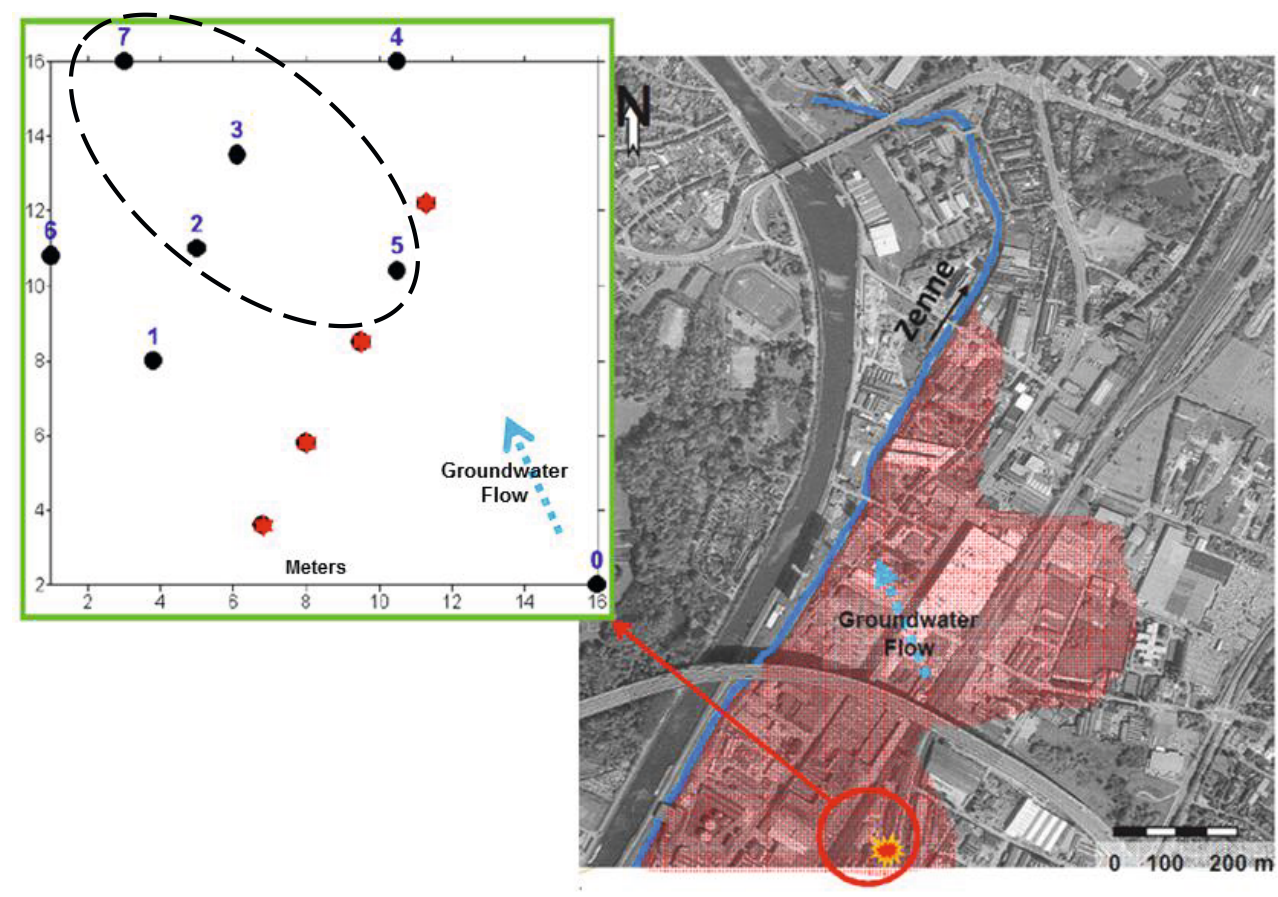

Figure S6.1 Schematic presentation of the study site close to the Zenne River. Monitoring wells (black circles) and injection wells used for glycerol delivery (red stars) at the site are indicated. Well 0 , located upstream of the injection wells, is taken as control. Samples were taken from the monitoring wells impacted by glycerol injection (shown in the dashed oval) i.e. well 2, 3, 5 and 7 (only shallow filter, 7A) as well as for the control well throughout the study. 
$\frac{6}{\text { 苛 }}$
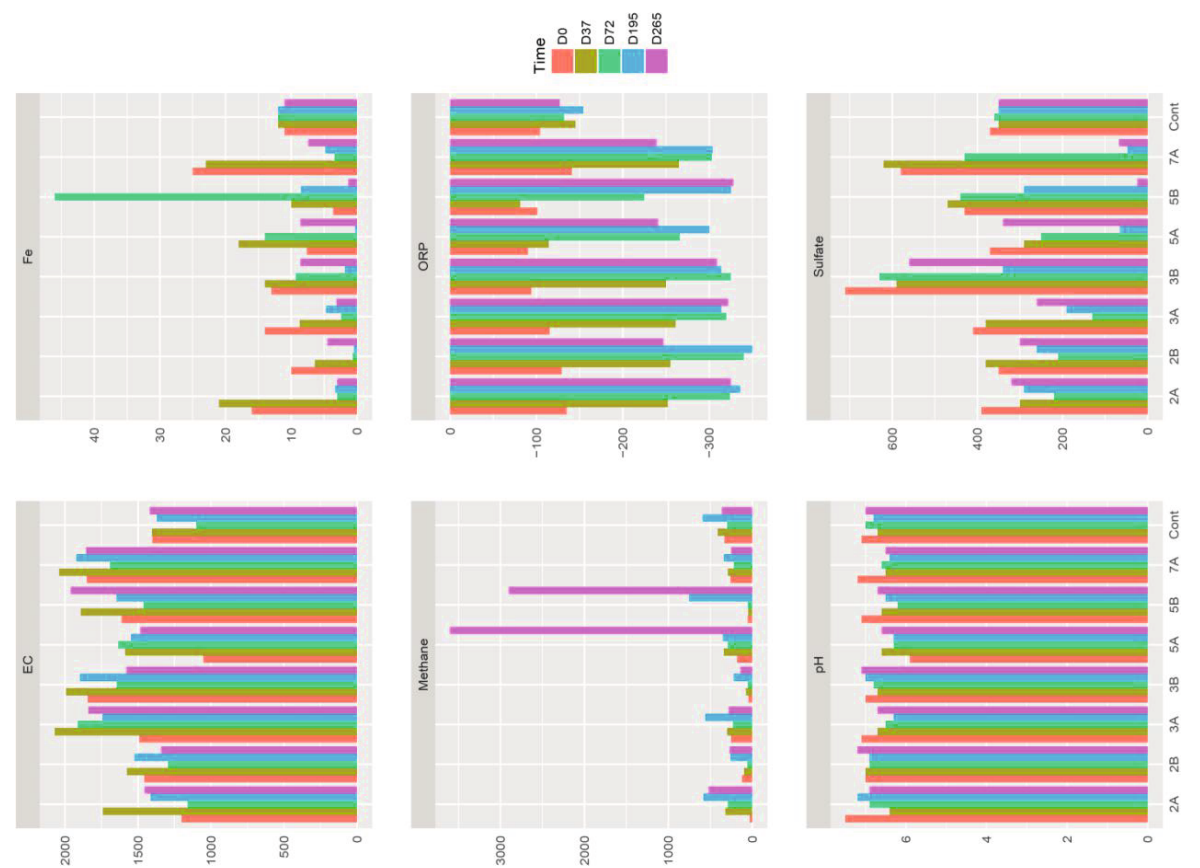

身
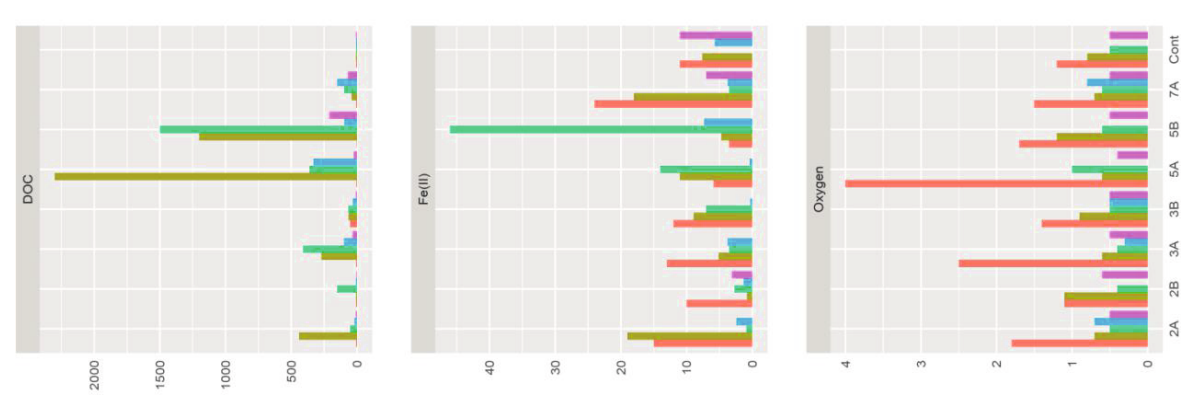

它

8

อ

ชี

ठ ह

吅

品

흘

गे

:

๑๋

के 犬े

离完

호

के क्षि

त्

就

एँ

클

뜰. 옹

ฮี

ह

용

on

荌

害

ठํ

กู.

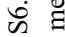

兽 
Geochemical and microbial analysis of groundwater at a site with biostimulated reductive dechlorination
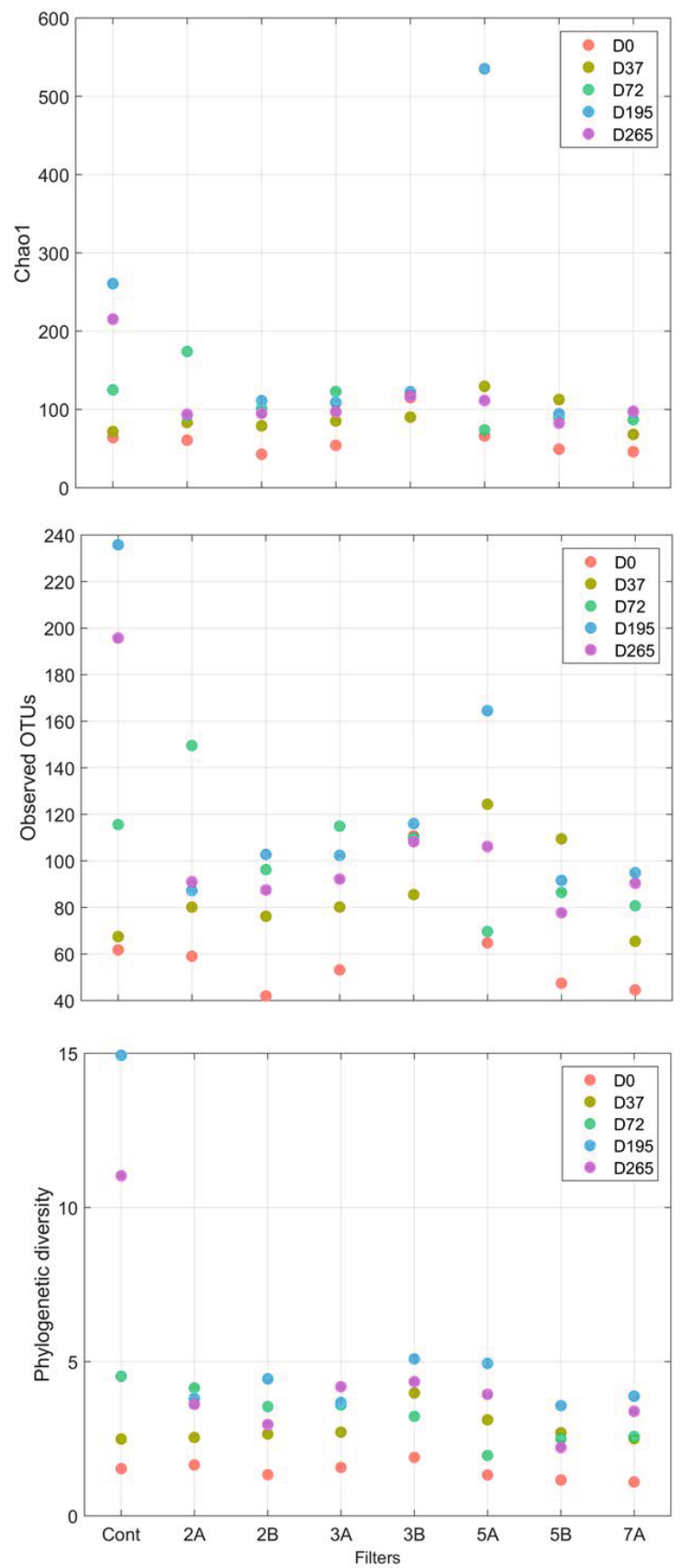

Figure S6.3 Predicted (Chao 1) and observed OTU richness, and phylogenetic diversity at each filter over time (five samples per filter). Cont: control well. 


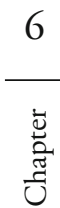

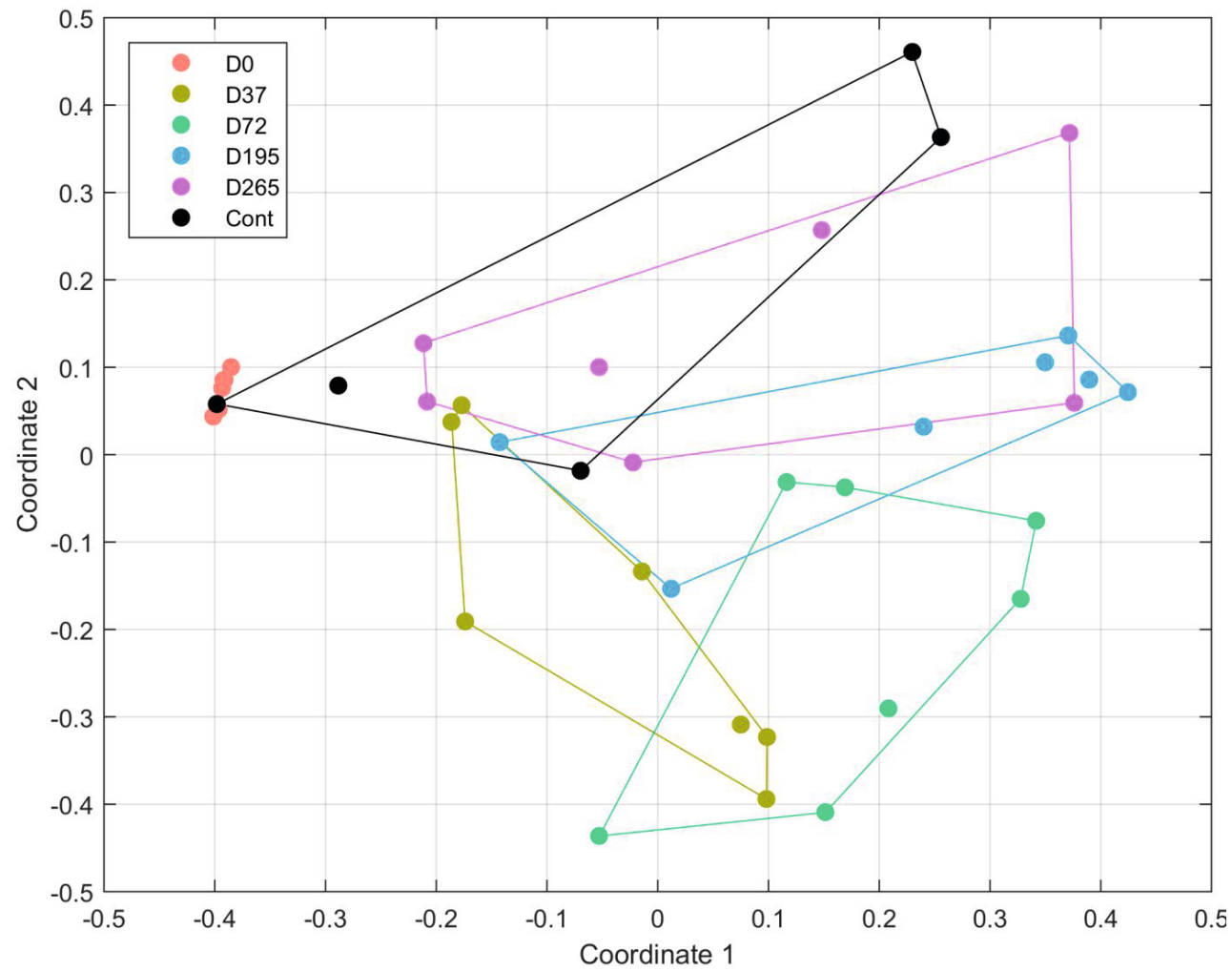

Figure S6.4 Ordination of community composition by nonmetric multidimensional scaling (NMDS) based on Bray-Curtis distances. Cont: control well. 


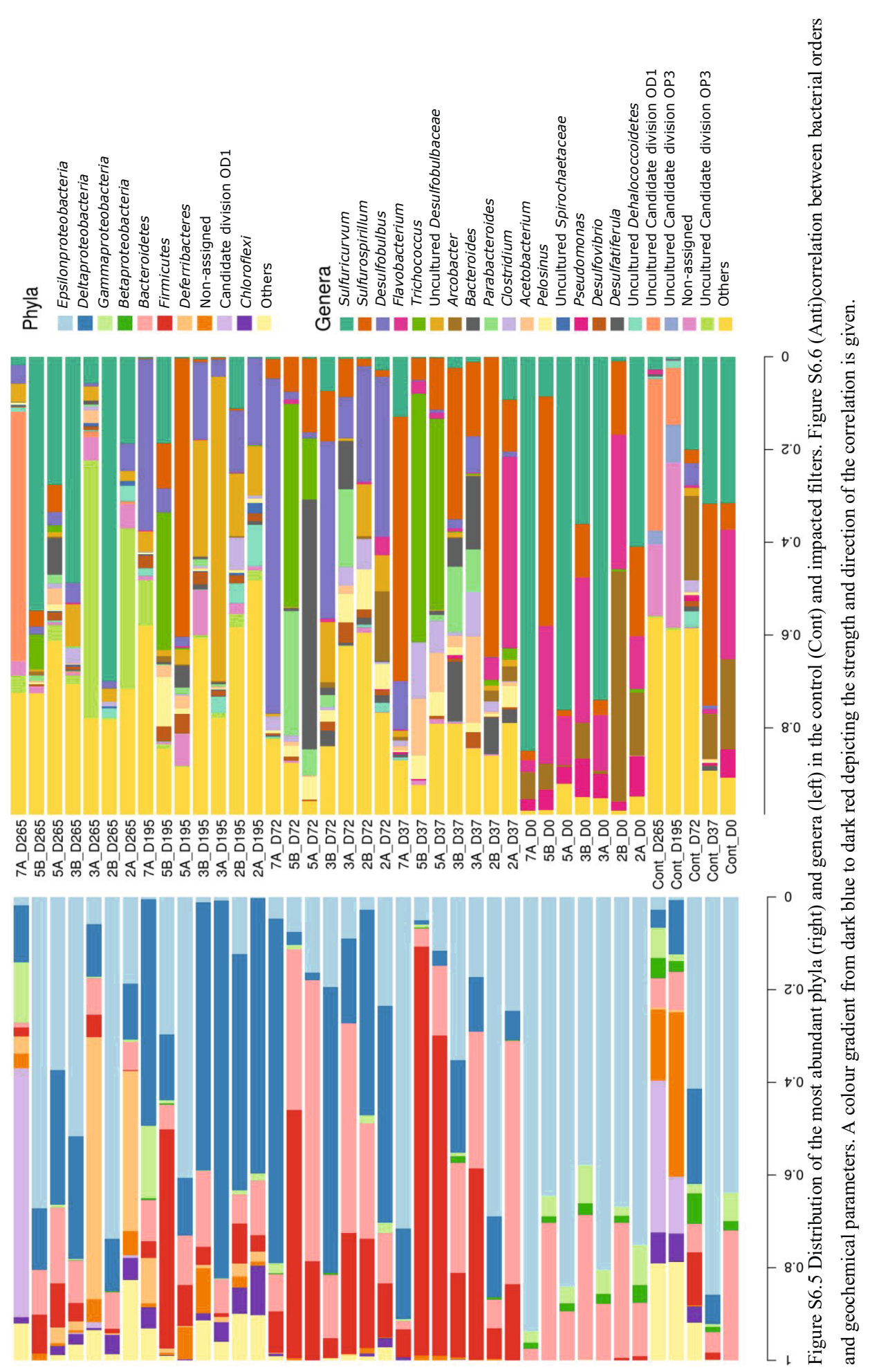




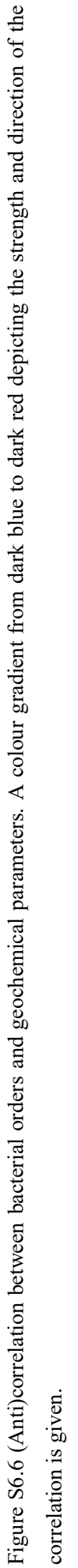


Geochemical and microbial analysis of groundwater at a site with biostimulated reductive dechlorination

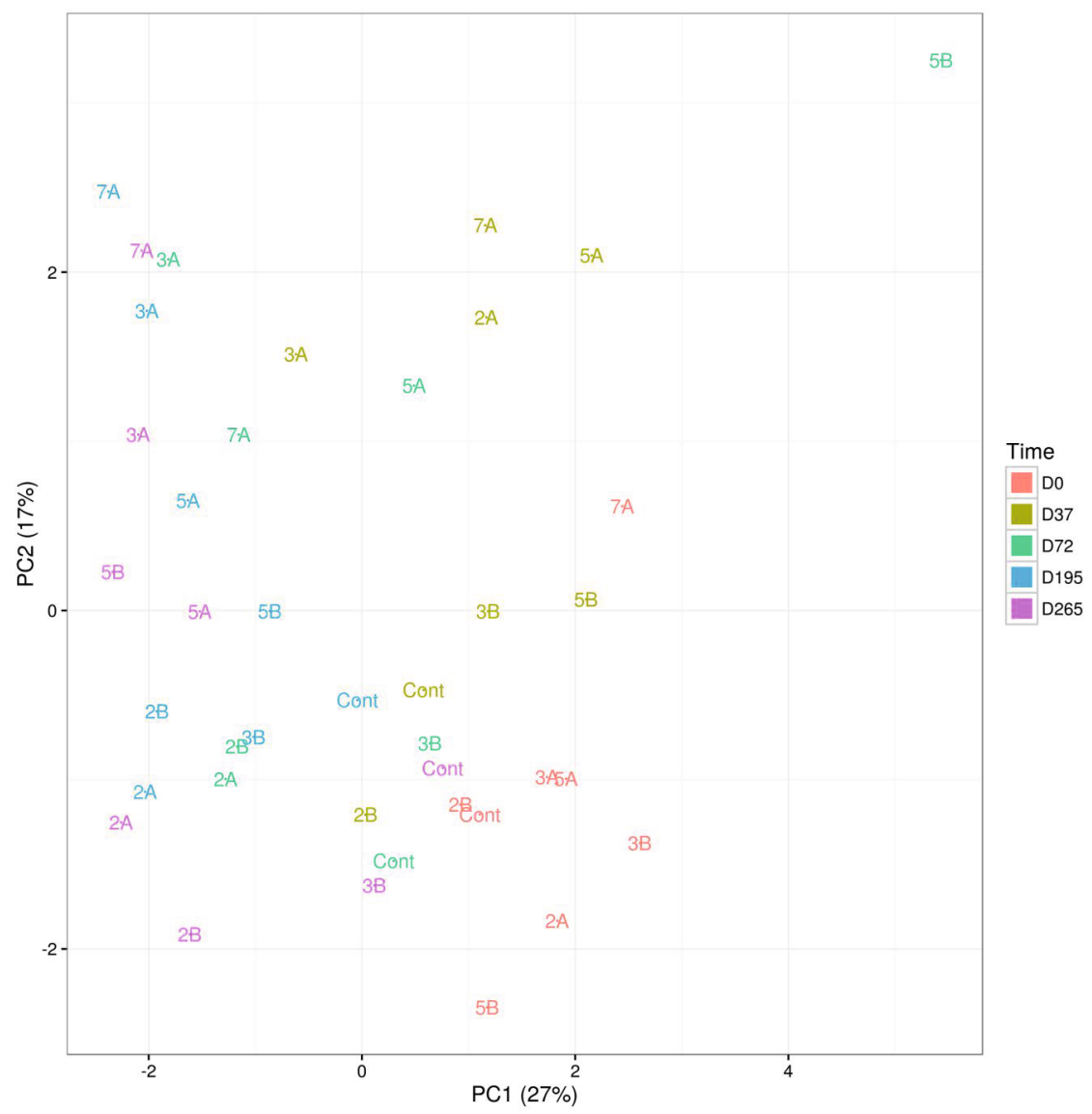

Figure S6.7 Principal component analysis (PCA) of geochemical parameters in the control (Cont) and impacted filters. 


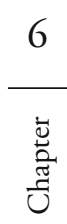
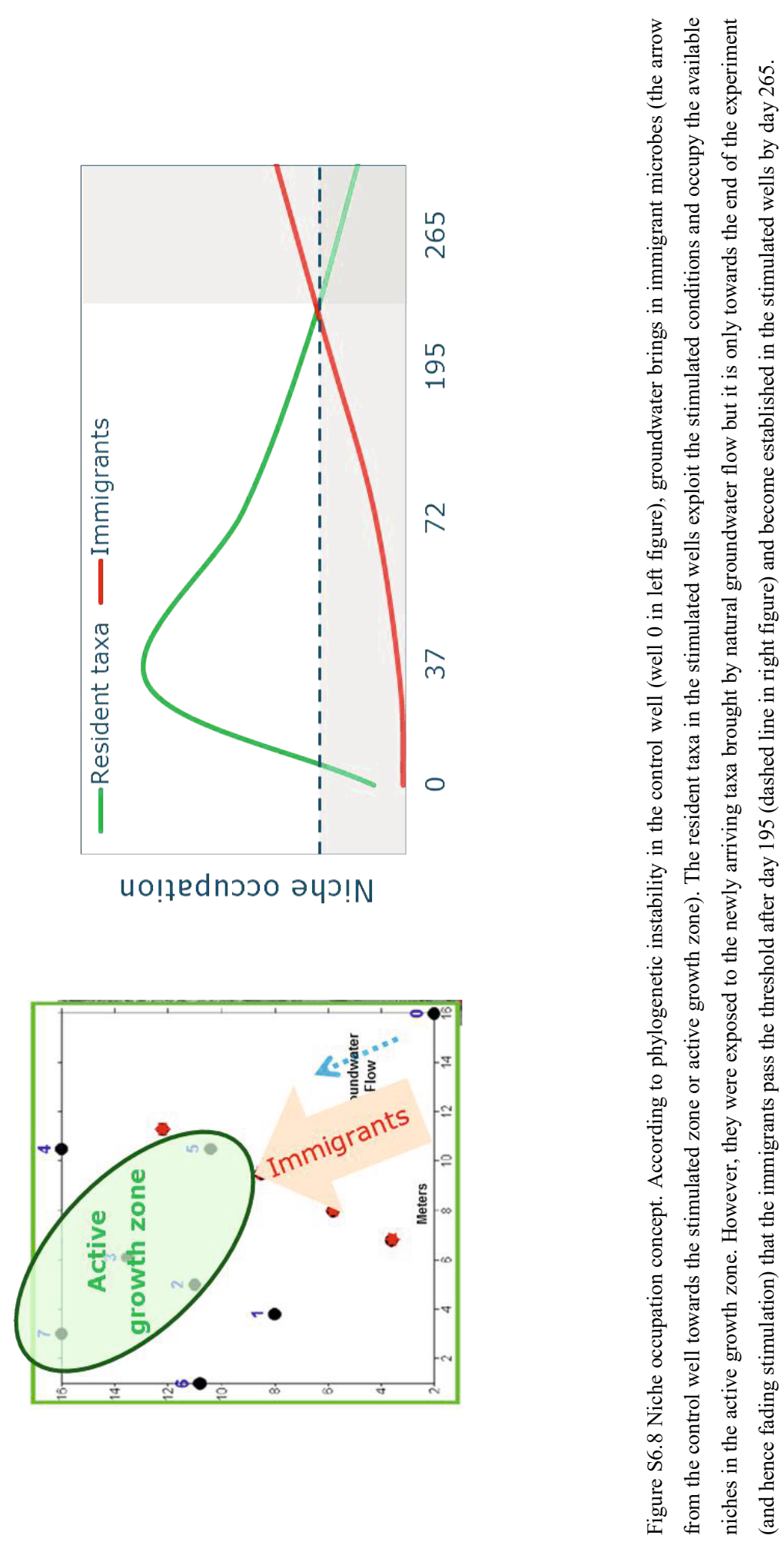

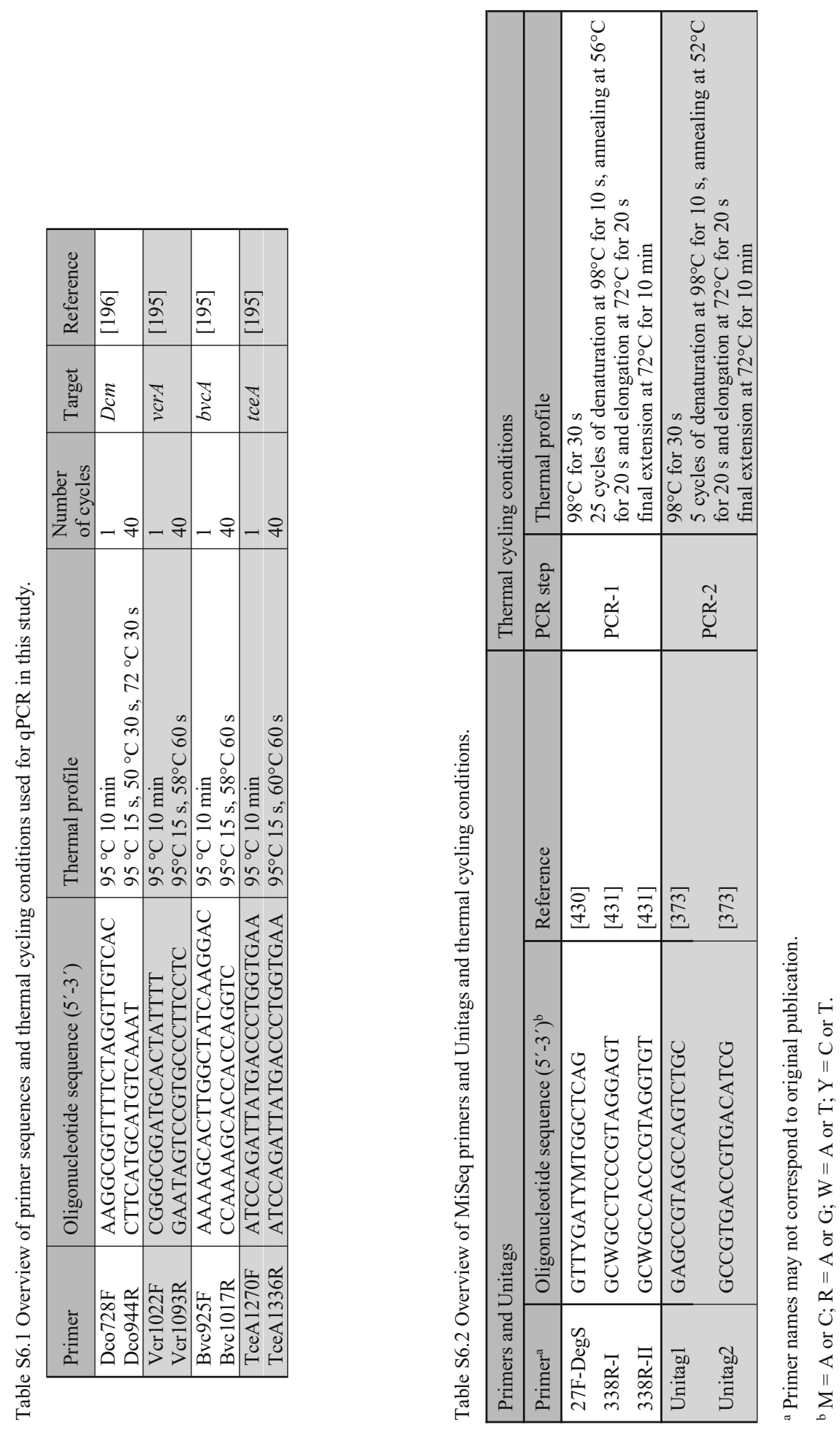

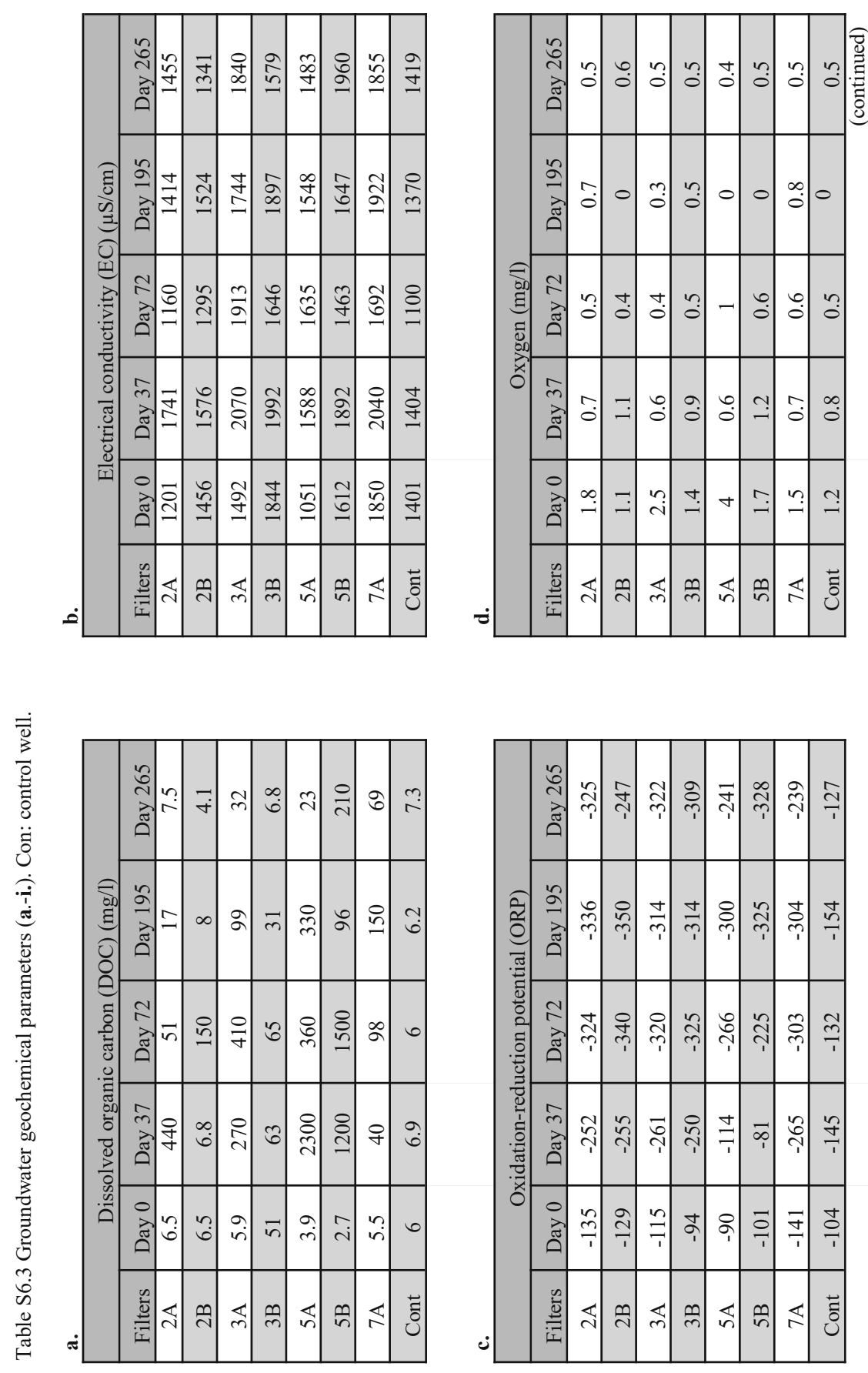

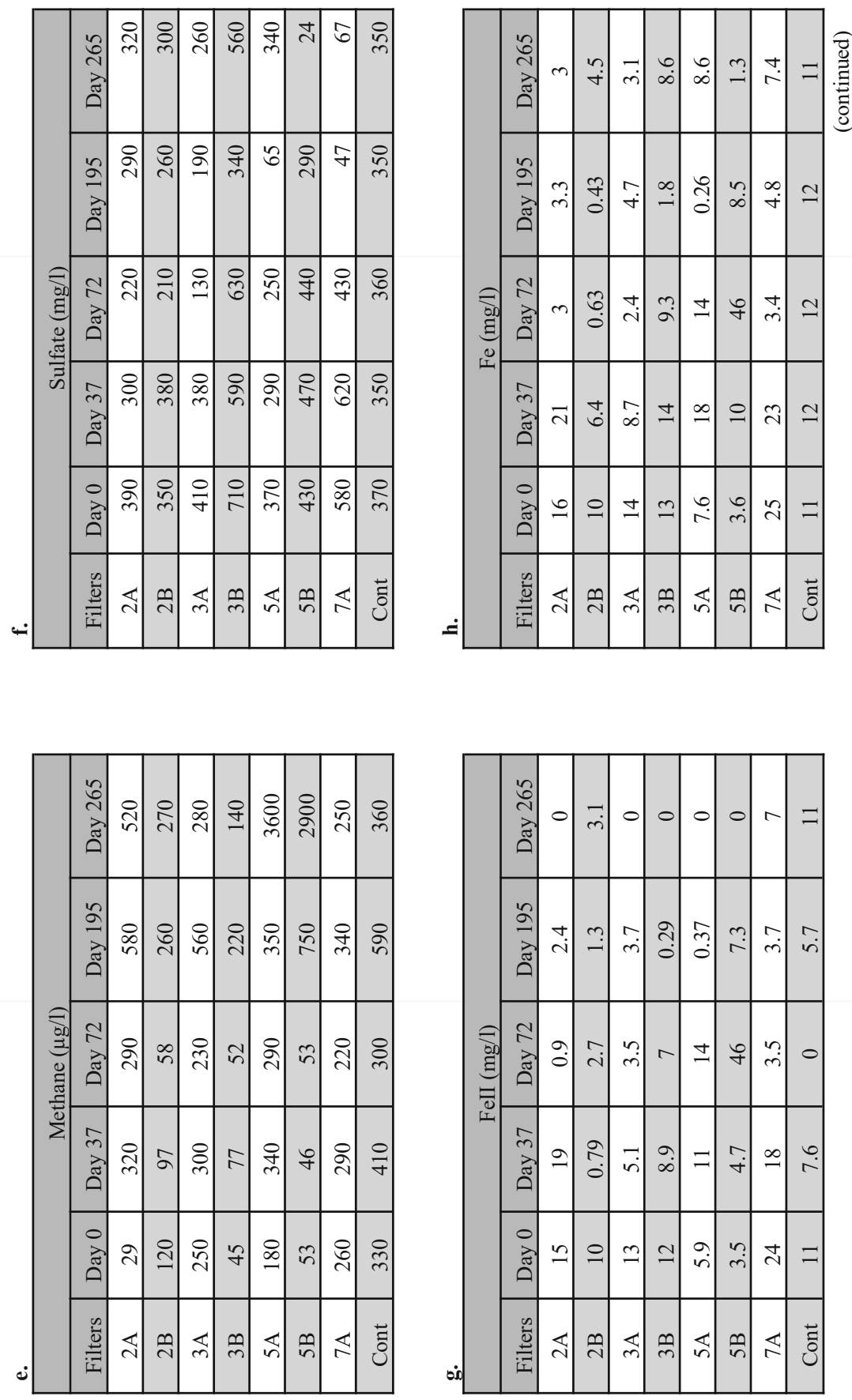


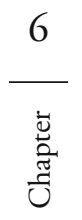

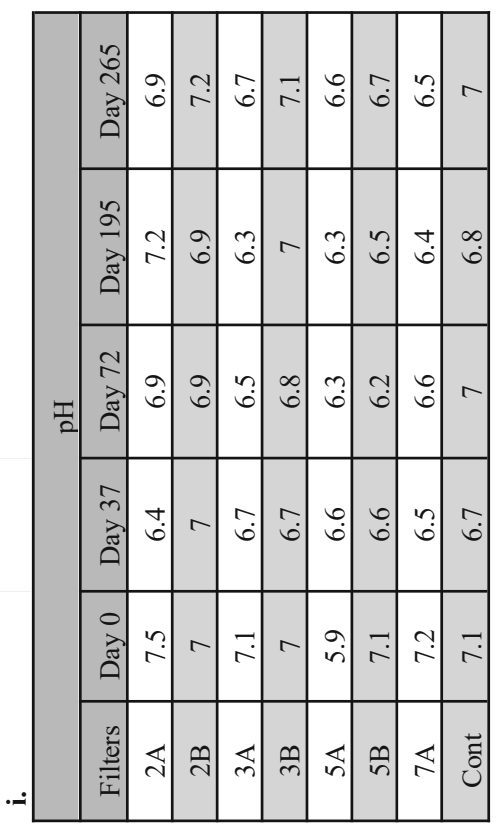


Table S6.4 Concentrations of TCE, cDCE, VC, ethene and $\delta^{13} \mathrm{C}$ of $\mathrm{cDCE}$ and VC and concentration-weighted average values of the isotope signature $\left(\delta^{13} \mathrm{C}_{\Sigma(\mathrm{CE})}\right)$.

a.

\begin{tabular}{|c|c|c|c|c|c|c|}
\hline Filters & Concentrations & Day 0 & Day 37 & Day 72 & Day 195 & Day 265 \\
\hline \multirow{7}{*}{$2 \mathrm{~A}$} & $\mathrm{TCE}(\mu \mathrm{mol} / \mathrm{L})$ & 0.5 & 0.3 & 0.0 & 0.0 & 0.0 \\
\hline & $\mathrm{cDCE}(\mu \mathrm{mol} / \mathrm{L})$ & 134.2 & 113.5 & 101.1 & 77.4 & 29.9 \\
\hline & $\mathrm{VC}(\mu \mathrm{mol} / \mathrm{L})$ & 24.0 & 24.0 & 27.2 & 65.6 & 48.0 \\
\hline & ETH $(\mu \mathrm{mol} / \mathrm{L})$ & 1.9 & 2.0 & 1.4 & 7.1 & 10.7 \\
\hline & $\delta^{13} \mathrm{C} \operatorname{cDCE}(\%)$ & -19.7 & NA & NA & -7.9 & -8.2 \\
\hline & $\delta^{13} \mathrm{C}$ VC (\%) & -33.5 & NA & NA & -35 & -22 \\
\hline & $\delta^{13} \mathrm{C}_{\text {¿CE }}(\%)$ & -21.8 & NA & NA & -20.5 & -15.7 \\
\hline \multirow{7}{*}{$2 \mathrm{~B}$} & $\mathrm{TCE}(\mu \mathrm{mol} / \mathrm{L})$ & 0.0 & 0.0 & 0.0 & 0.0 & 0.0 \\
\hline & $\operatorname{cDCE}(\mu \mathrm{mol} / \mathrm{L})$ & 103.2 & 98.0 & 92.9 & 56.8 & 44.4 \\
\hline & $\mathrm{VC}(\mu \mathrm{mol} / \mathrm{L})$ & 12.5 & 3.7 & 16.0 & 38.4 & 41.6 \\
\hline & $\mathrm{ETH}(\mu \mathrm{mol} / \mathrm{L})$ & 2.4 & 0.5 & 0.5 & 3.0 & 11.4 \\
\hline & $\delta^{13} \mathrm{C}$ cDCE (\%o) & -19.3 & NA & NA & -14.4 & 1.3 \\
\hline & $\delta^{13} \mathrm{C} \mathrm{VC}(\%)$ & -27 & NA & NA & -32 & -33.0 \\
\hline & $\delta^{13} \mathrm{C}_{\text {ECE }}(\% 0)$ & -20.2 & NA & NA & -21.4 & -15.2 \\
\hline \multirow{7}{*}{$3 \mathrm{~A}$} & $\mathrm{TCE}(\mu \mathrm{mol} / \mathrm{L})$ & 0.5 & 0.4 & 0.0 & 0.0 & 0.0 \\
\hline & $\operatorname{cDCE}(\mu \mathrm{mol} / \mathrm{L})$ & 165.1 & 206.4 & 227.0 & 185.8 & 123.8 \\
\hline & $\mathrm{VC}(\mu \mathrm{mol} / \mathrm{L})$ & 30.4 & 48.0 & 54.4 & 67.2 & 96.0 \\
\hline & $\mathrm{ETH}(\mu \mathrm{mol} / \mathrm{L})$ & 2.4 & 2.4 & 1.6 & 3.4 & 1.8 \\
\hline & $\delta^{13} \mathrm{C} \mathrm{cDCE}(\%)$ & -20.0 & NA & NA & -16.6 & -11.1 \\
\hline & $\delta^{13} \mathrm{C}$ VC (\%o) & -30 & NA & NA & -37.2 & -38.5 \\
\hline & $\delta^{13} \mathrm{C}_{\text {ECE }}(\%)$ & -21.5 & NA & NA & -22.1 & -20.0 \\
\hline \multirow{7}{*}{ 3B } & $\mathrm{TCE}(\mu \mathrm{mol} / \mathrm{L})$ & 0.0 & 0.0 & 0.0 & 0.0 & 0.0 \\
\hline & $\mathrm{cDCE}(\mu \mathrm{mol} / \mathrm{L})$ & 21.7 & 49.5 & 29.9 & 39.2 & 14.4 \\
\hline & $\mathrm{VC}(\mu \mathrm{mol} / \mathrm{L})$ & 5.6 & 11.0 & 6.9 & 14.2 & 8.5 \\
\hline & $\mathrm{ETH}(\mu \mathrm{mol} / \mathrm{L})$ & 0.0 & 0.5 & 0.2 & 1.1 & 0.3 \\
\hline & $\delta^{13} \mathrm{C} \mathrm{cDCE}(\%)$ & -18.8 & NA & NA & -16.9 & -9.1 \\
\hline & $\delta^{13} \mathrm{C} \mathrm{VC} \mathrm{( \% )}$ & -33.6 & NA & NA & -29.9 & -38.7 \\
\hline & $\delta^{13} \mathrm{C}_{\text {¿CE }}(\%)$ & -21.8 & NA & NA & -20.3 & -19.2 \\
\hline \multirow{7}{*}{$5 \mathrm{~A}$} & $\mathrm{TCE}(\mu \mathrm{mol} / \mathrm{L})$ & 1.0 & 0.9 & 0.0 & 0.0 & 0.6 \\
\hline & $\mathrm{cDCE}(\mu \mathrm{mol} / \mathrm{L})$ & 113.5 & 123.8 & 113.5 & 103.2 & 123.8 \\
\hline & $\mathrm{VC}(\mu \mathrm{mol} / \mathrm{L})$ & 24.0 & 22.4 & 25.6 & 6.1 & 25.6 \\
\hline & $\mathrm{ETH}(\mu \mathrm{mol} / \mathrm{L})$ & 1.7 & 1.8 & 1.6 & 0.8 & 1.9 \\
\hline & $\delta^{13} \mathrm{C}$ cDCE (\%o) & -20.2 & NA & NA & -18.8 & -18.2 \\
\hline & $\delta^{13} \mathrm{C} \mathrm{VC} \mathrm{( \% o)}$ & -33.0 & NA & NA & -36.7 & -37.4 \\
\hline & $\delta^{13} \mathrm{C}_{\text {ICE }}(\%)$ & -22.4 & NA & NA & -22.5 & -20.8 \\
\hline \multirow{5}{*}{$5 \mathrm{~B}$} & $\mathrm{TCE}(\mu \mathrm{mol} / \mathrm{L})$ & 0.0 & 0.0 & 0.0 & 0.7 & 0.0 \\
\hline & $\mathrm{cDCE}(\mu \mathrm{mol} / \mathrm{L})$ & 2.1 & 12.4 & 19.6 & 14.4 & 9.6 \\
\hline & $\mathrm{VC}(\mu \mathrm{mol} / \mathrm{L})$ & 0.4 & 1.6 & 3.5 & 4.0 & 3.4 \\
\hline & $\mathrm{ETH}(\mu \mathrm{mol} / \mathrm{L})$ & 0.4 & 0.2 & 0.1 & 3.9 & 0.5 \\
\hline & $\delta^{13} \mathrm{C}$ cDCE $(\%)$ & 3.6 & A & NA & -18.9 & -17.4 \\
\hline
\end{tabular}

(continued) 


\begin{tabular}{|c|c|c|c|c|c|c|}
\hline & $\delta^{13} \mathrm{C} \mathrm{VC} \mathrm{( \% o)}$ & -27.6 & NA & NA & -34.6 & -34.3 \\
\hline & $\delta^{13} \mathrm{C}_{\text {ECE }}(\%)$ & -20.2 & NA & NA & -22.3 & -21.6 \\
\hline \multirow{7}{*}{$7 \mathrm{~A}$} & $\mathrm{TCE}(\mu \mathrm{mol} / \mathrm{L})$ & 0.1 & 0.3 & 0.0 & 0.0 & 0.0 \\
\hline & $\mathrm{cDCE}(\mu \mathrm{mol} / \mathrm{L})$ & 207.3 & 207.3 & 228.0 & 217.6 & 124.4 \\
\hline & $\mathrm{VC}(\mu \mathrm{mol} / \mathrm{L})$ & 51.2 & 57.6 & 65.6 & 100.8 & 131.2 \\
\hline & $\mathrm{ETH}(\mu \mathrm{mol} / \mathrm{L})$ & 2.5 & 2.4 & 1.9 & 3.1 & 3.5 \\
\hline & $\delta^{13} \mathrm{C}$ cDCE (\%o) & -18.7 & NA & NA & -14.7 & -5.6 \\
\hline & $\delta^{13} \mathrm{C} \mathrm{VC} \mathrm{( \% o)}$ & -34.9 & NA & NA & -40.2 & -36.8 \\
\hline & $\delta^{13} \mathrm{C}_{\text {¿CE }}(\%)$ & -21.9 & NA & NA & -22.8 & -16.2 \\
\hline \multirow{7}{*}{ Control } & $\mathrm{TCE}(\mu \mathrm{mol} / \mathrm{L})$ & 2.2 & 2.5 & 2.4 & 1.9 & 1.1 \\
\hline & $\operatorname{cDCE}(\mu \mathrm{mol} / \mathrm{L})$ & 113.5 & 123.8 & 134.2 & 123.8 & 100.1 \\
\hline & $\mathrm{VC}(\mu \mathrm{mol} / \mathrm{L})$ & 24.0 & 20.8 & 22.4 & 22.4 & 16.0 \\
\hline & $\mathrm{ETH}(\mu \mathrm{mol} / \mathrm{L})$ & 1.9 & 2.1 & 1.6 & 3.4 & 1.9 \\
\hline & $\delta^{13} \mathrm{C}$ cDCE (\%o) & -19.9 & NA & NA & -20.5 & -20.0 \\
\hline & $\delta^{13} \mathrm{C} \mathrm{VC} \mathrm{( \% )}$ & -32.4 & NA & NA & -31 & -32 \\
\hline & $\delta^{13} \mathrm{C}_{\Sigma \mathrm{CE}}(\% \mathrm{0})$ & -22.1 & NA & NA & -22.1 & -21.9 \\
\hline
\end{tabular}

6

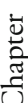




\begin{tabular}{|c|c|c|c|c|c|c|c|c|c|c|c|c|c|c|c|c|c|c|c|c|c|c|}
\hline \multirow{2}{*}{\multicolumn{2}{|c|}{ 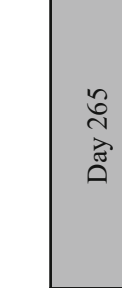 }} & 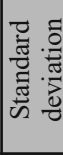 & 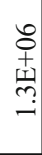 & $\begin{array}{l}\text { ○े } \\
+ \\
\text { 至 } \\
\end{array}$ & 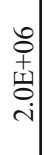 & 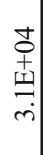 & $\begin{array}{c}\hat{0} \\
+ \\
\text { 11 } \\
\infty \\
-\end{array}$ & 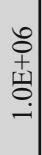 & $\begin{array}{l}0 \\
0 \\
+ \\
\text { 音 } \\
-1\end{array}$ & $\begin{array}{l}8 \\
\vdots \\
\text { ज⿱ } \\
0 \\
0\end{array}$ & 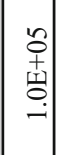 & $\begin{array}{c}0 \\
+ \\
+ \\
0 \\
0 \\
-\end{array}$ & $\begin{array}{l}+ \\
0 \\
+ \\
+ \\
0 \\
\dot{r}\end{array}$ & $\begin{array}{l}m \\
0 \\
+ \\
\text { ț } \\
\dot{\nabla} \\
\end{array}$ & 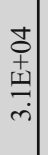 & $\begin{array}{l}\text { ㅇ } \\
+ \\
\text { I } \\
\vdots \\
0\end{array}$ & $\begin{array}{l}+ \\
+ \\
\pm \\
\stackrel{1}{0} \\
\infty\end{array}$ & $\begin{array}{l}\text { ô } \\
\vdots \\
\text { Iل } \\
-\end{array}$ & $\begin{array}{l}n \\
0 \\
+ \\
0 \\
\vdots \\
i\end{array}$ & 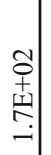 & $\begin{array}{c}+ \\
+ \\
+ \\
0 \\
0 \\
m\end{array}$ & $\begin{array}{l}8 \\
\vdots \\
+ \\
\text { †े } \\
0\end{array}$ \\
\hline & & نे & $\begin{array}{l}\hat{0} \\
+ \\
\text { 至 } \\
-\end{array}$ & $\begin{array}{c}0 \\
0 \\
+ \\
\text { t⿹ } \\
\text { r. }\end{array}$ & $\begin{array}{c}\hat{0} \\
+ \\
\text { tà } \\
\text { - }\end{array}$ & 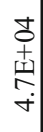 & 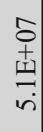 & $\begin{array}{c}5 \\
0 \\
+ \\
\text { ஸे } \\
\dot{0}\end{array}$ & 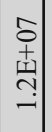 & $\begin{array}{l}8 \\
+ \\
+ \\
0 \\
0 \\
0\end{array}$ & 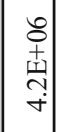 & 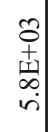 & $\begin{array}{l}n \\
0 \\
+ \\
+1 \\
r \\
\dot{m}\end{array}$ & $\begin{array}{l}m \\
⿱ 亠 \\
+ \\
= \\
m \\
m\end{array}$ & $\begin{array}{l}n \\
0 \\
+ \\
\text { In } \\
\text { n். }\end{array}$ & \begin{tabular}{l|l}
8 \\
+ \\
+ \\
†े \\
0
\end{tabular} & 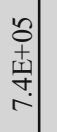 & $\begin{array}{c}\text { ఇ } \\
+ \\
\pm \\
= \\
-\end{array}$ & $\begin{array}{c}n \\
0 \\
+ \\
=0 \\
m \\
\infty\end{array}$ & 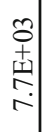 & 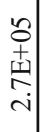 & $\begin{array}{l}8 \\
+ \\
+1 \\
0 \\
0\end{array}$ \\
\hline \multirow{2}{*}{\multicolumn{2}{|c|}{$\begin{array}{l}\frac{n}{a} \\
\frac{n}{a}\end{array}$}} & 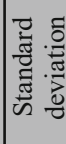 & $\begin{array}{l}\stackrel{8}{+} \\
+ \\
\stackrel{1}{n} \\
\end{array}$ & $\begin{array}{l}2 \\
\vdots \\
+ \\
0 \\
0 \\
6\end{array}$ & 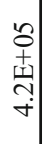 & 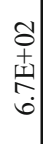 & $\begin{array}{l}8 \\
\vdots \\
+1 \\
= \\
=\end{array}$ & $\begin{array}{l}n \\
0 \\
\pm \\
\text { m. } \\
\end{array}$ & 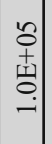 & $\begin{array}{l}+ \\
0 \\
+ \\
\text { ज्ञ } \\
\text { m. }\end{array}$ & 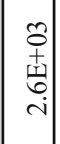 & 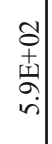 & 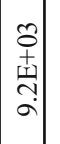 & 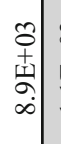 & $\begin{array}{l}0 \\
0 \\
+ \\
= \\
= \\
=\end{array}$ & 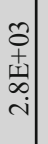 & 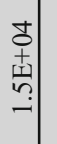 & 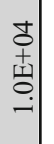 & 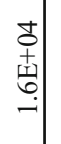 & $\begin{array}{l}\text { ô } \\
1 \\
\text { ț } \\
\infty \\
\infty\end{array}$ & $\begin{array}{c} \pm \\
+ \\
\pm \\
\\
\infty\end{array}$ & 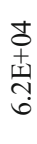 \\
\hline & & نे & $\begin{array}{c}\circ \\
+ \\
+ \\
+1 \\
\infty \\
\dot{m}\end{array}$ & $\begin{array}{c}0 \\
0 \\
+ \\
+1 \\
n \\
n\end{array}$ & \begin{tabular}{c}
0 \\
$\vdots$ \\
+ \\
\multicolumn{1}{|c}{} \\
m.
\end{tabular} & $\begin{array}{l}n \\
+ \\
+1 \\
\sim \\
\infty\end{array}$ & 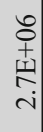 & 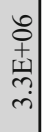 & $\begin{array}{l}0 \\
\vdots \\
\pm \\
\pm \\
= \\
-1\end{array}$ & $\begin{array}{l}\dot{+} \\
+ \\
+ \\
+ \\
\forall\end{array}$ & 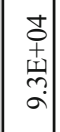 & $\begin{array}{c}0 \\
0 \\
+ \\
+ \\
0 \\
-\end{array}$ & $\begin{array}{c}+ \\
+ \\
+ \\
\pm \\
\infty \\
+\end{array}$ & $\begin{array}{l}+ \\
+ \\
+ \\
\text { ज్ } \\
- \\
-\end{array}$ & 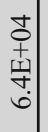 & $\begin{array}{l}m \\
0 \\
+ \\
\text { I } \\
\dot{r}\end{array}$ & $\begin{array}{l}n \\
0 \\
+ \\
\text { I⿱ } \\
-1\end{array}$ & 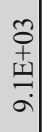 & $\begin{array}{l}+ \\
0 \\
+ \\
\text { t } \\
0 \\
0\end{array}$ & 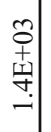 & $\begin{array}{l}0 \\
+ \\
+ \\
\text { ț } \\
-1\end{array}$ & $\begin{array}{l} \pm \\
+ \\
+ \\
+ \\
+ \\
+\end{array}$ \\
\hline \multirow{2}{*}{\multicolumn{2}{|c|}{ 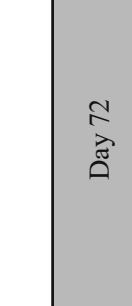 }} & 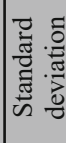 & 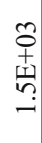 & 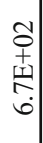 & 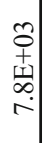 & $\begin{array}{l}8 \\
+ \\
+1 \\
0 \\
0 \\
0\end{array}$ & 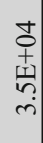 & 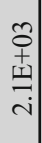 & $\left|\begin{array}{c}+ \\
0 \\
+ \\
w \\
\vdots \\
i\end{array}\right|$ & $\begin{array}{l}\Re \\
0 \\
0 \\
0 \\
0 \\
0 \\
0\end{array}$ & 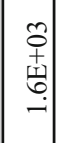 & 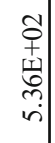 & 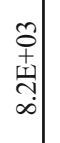 & 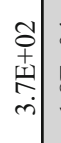 & 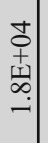 & $\begin{array}{c}8 \\
0 \\
+ \\
\text { 미 } \\
0\end{array}$ & $\begin{array}{l}0 \\
0 \\
+ \\
\pm 0 \\
- \\
-1\end{array}$ & 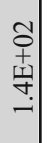 & $\begin{array}{c}\tilde{0} \\
+ \\
\pm \\
m \\
-\end{array}$ & \begin{tabular}{c}
0 \\
0 \\
+ \\
\multirow{2}{1}{} \\
-1
\end{tabular} & $\begin{array}{c}m \\
+ \\
+ \\
\text { 至 } \\
\end{array}$ & 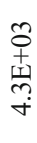 \\
\hline & & $\begin{array}{l}\overrightarrow{0} \\
\stackrel{0}{0} \\
0\end{array}$ & $\begin{array}{c} \pm \\
+ \\
+ \\
+1 \\
\infty \\
-\end{array}$ & 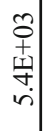 & $\begin{array}{l}\qquad \\
0 \\
+ \\
\pm \\
= \\
=\end{array}$ & $\begin{array}{l}8 \\
8 \\
+ \\
\text { 딩 } \\
\dot{0}\end{array}$ & $\begin{array}{l} \pm \\
+ \\
+ \\
\pm \\
\dot{H}\end{array}$ & $\begin{array}{c}n \\
0 \\
+ \\
\pm n \\
n \\
m\end{array}$ & 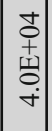 & $\begin{array}{l}\tilde{0} \\
+ \\
\pm \\
\infty \\
-\end{array}$ & $\mid \begin{array}{c} \pm \\
0 \\
+ \\
\pm \\
m \\
\\
\mid\end{array}$ & $\begin{array}{c}0 \\
0 \\
+ \\
\text { İ } \\
- \\
-\end{array}$ & $\begin{array}{c}+ \\
+ \\
+ \\
\pm \\
\infty \\
-\end{array}$ & 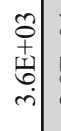 & $\begin{array}{c}+ \\
0 \\
+ \\
\pm \\
\infty \\
i\end{array}$ & $\begin{array}{l}8 \\
8 \\
+ \\
\text { İ } \\
0 \\
0\end{array}$ & $\begin{array}{c} \pm \\
+ \\
\pm \\
\pm \\
\dot{1}\end{array}$ & $\begin{array}{l}0 \\
0 \\
+ \\
\text { t్d } \\
-\end{array}$ & $\begin{array}{c}+ \\
+ \\
+ \\
+1 \\
+ \\
\dot{r}\end{array}$ & 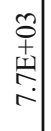 & $\begin{array}{c}+ \\
+ \\
+ \\
\text { गे } \\
i\end{array}$ & 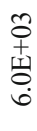 \\
\hline \multirow{2}{*}{\multicolumn{2}{|c|}{$\begin{array}{l}\text { m } \\
\text { बે }\end{array}$}} & 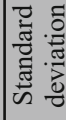 & $\begin{array}{l} \pm \\
+ \\
+ \\
+\infty \\
\infty \\
-\end{array}$ & $\begin{array}{c}m \\
0 \\
+ \\
\pm=1 \\
\dot{1}\end{array}$ & 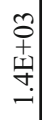 & \begin{tabular}{l}
$m$ \\
+ \\
+ \\
$=1$ \\
\hdashline
\end{tabular} & $\begin{array}{l} \pm \\
+ \\
+ \\
\pm \\
\forall\end{array}$ & $\begin{array}{c}m \\
0 \\
+ \\
\text { ஸు } \\
\text { m. }\end{array}$ & 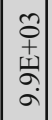 & $\begin{array}{c}\tilde{0} \\
+ \\
\pm \\
\tilde{+} \\
\dot{+}\end{array}$ & $\begin{array}{l}\tilde{0} \\
+ \\
+ \\
+ \\
\dot{\gamma} \\
\dot{\nabla}\end{array}$ & $\begin{array}{l}8 \\
\vdots \\
+1 \\
0 \\
0 \\
0\end{array}$ & 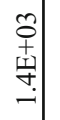 & $\begin{array}{c}m \\
0 \\
+ \\
\text { tà } \\
\dot{m}\end{array}$ & 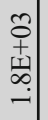 & $\begin{array}{c}8 \\
8 \\
+ \\
\text { 19 } \\
\dot{0}\end{array}$ & $\begin{array}{l}\overrightarrow{0} \\
+ \\
+1 \\
\dot{y} \\
i\end{array}$ & 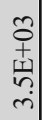 & 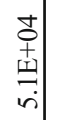 & 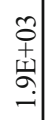 & 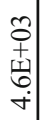 & 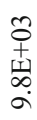 \\
\hline & & 롱 & $\begin{array}{l}+ \\
+ \\
+ \\
\text { to } \\
\forall\end{array}$ & $\begin{array}{l}+ \\
+ \\
+ \\
+ \pm \\
\stackrel{+}{+}\end{array}$ & $\begin{array}{l}+ \\
+ \\
+ \\
\pm \\
\infty \\
i\end{array}$ & 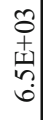 & 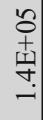 & $\begin{array}{l} \pm \\
+ \\
+ \\
\pm \\
= \\
=\end{array}$ & $\left|\begin{array}{c}n \\
0 \\
+1 \\
1 \\
0 \\
\infty\end{array}\right|$ & $\begin{array}{l} \pm \\
+ \\
+ \\
= \\
=\end{array}$ & $\begin{array}{c}+ \\
0 \\
+ \\
\pm 1 \\
\infty \\
-1 \\
-1\end{array}$ & $\begin{array}{l}8 \\
+ \\
+ \\
\text { 1 } \\
\vdots \\
0\end{array}$ & $\begin{array}{c} \pm \\
+ \\
+ \\
\pm \\
\vdots \\
\infty\end{array}$ & 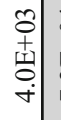 & $\begin{array}{c} \pm \\
+ \\
+ \\
\text { ஸे } \\
r \\
r\end{array}$ & \begin{tabular}{l|l}
8 \\
+ \\
+ \\
1 \\
0 \\
0
\end{tabular} & 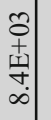 & 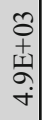 & $\begin{array}{l}0 \\
+ \\
+ \\
+1 \\
\infty \\
i\end{array}$ & 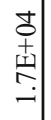 & 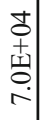 & 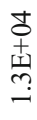 \\
\hline \multirow{4}{*}{ 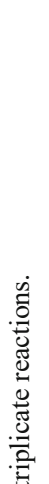 } & & 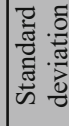 & $\begin{array}{c}m \\
0 \\
+ \\
\omega \\
\omega \\
\infty \\
\infty\end{array}$ & $\begin{array}{c}0 \\
0 \\
+ \\
+1 \\
\infty \\
-\end{array}$ & 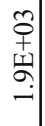 & 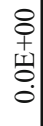 & 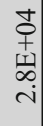 & $\begin{array}{c}3 \\
+ \\
+ \\
+\infty \\
\infty \\
\infty\end{array}$ & 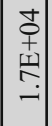 & $\begin{array}{c}+ \\
+ \\
+ \\
\text { In } \\
-\end{array}$ & $\begin{array}{c}\tilde{0} \\
+ \\
= \\
m \\
-\end{array}$ & $\begin{array}{l}0 \\
\vdots \\
+ \\
\text { İ } \\
\dot{0}\end{array}$ & 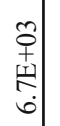 & 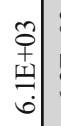 & 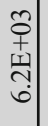 & $\begin{array}{c}8 \\
\vdots \\
+ \\
\text { 밓 } \\
0\end{array}$ & 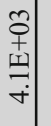 & 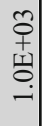 & 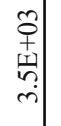 & $\begin{array}{c}8 \\
8 \\
+ \\
\text { 뭉 } \\
\dot{0}\end{array}$ & $\begin{array}{c} \pm \\
+ \\
+ \\
\text { ஸు } \\
-\end{array}$ & 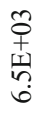 \\
\hline & & $\begin{array}{l}\frac{1}{4} \\
0 \\
0\end{array}$ & $\begin{array}{c} \pm \\
+ \\
+ \\
\pm \\
0 \\
\dot{n}\end{array}$ & $\begin{array}{l}n \\
0 \\
+ \\
\omega n \\
n \\
n\end{array}$ & $\begin{array}{c} \pm \\
+ \\
+ \\
\pm \\
\dot{m}\end{array}$ & 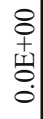 & $\begin{array}{l}n \\
+ \\
+ \\
\omega \\
\infty \\
-\end{array}$ & $\begin{array}{l} \pm \\
0 \\
+ \\
\pm 0 \\
\dot{0} \\
\dot{m}\end{array}$ & $\mid \begin{array}{c}n \\
0 \\
+ \\
\omega n \\
n \\
-1\end{array}$ & $\begin{array}{l} \pm \\
+ \\
+ \\
\pm \\
ت \\
-\end{array}$ & 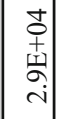 & $\begin{array}{l}8 \\
0 \\
+ \\
0 \\
0 \\
0\end{array}$ & 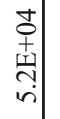 & $\begin{array}{l}m \\
⿱ \\
+ \\
\pm \\
m \\
\dot{+}\end{array}$ & $\begin{array}{l} \pm \\
+ \\
\pm \\
\text { ज्ञ } \\
-\end{array}$ & $\begin{array}{l}8 \\
8 \\
+ \\
\text { 甲్ } \\
0\end{array}$ & $\begin{array}{c}+ \\
0 \\
+ \\
\text { ते } \\
n\end{array}$ & 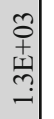 & $\begin{array}{l}+ \\
+ \\
+ \\
\pm 1 \\
+1 \\
-i\end{array}$ & $\begin{array}{c}8 \\
\vdots \\
+1 \\
\text { 잉 } \\
0\end{array}$ & 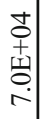 & $\begin{array}{l}\Re \\
0 \\
+ \\
\stackrel{1}{n} \\
r\end{array}$ \\
\hline & & & కే & \begin{tabular}{l}
$\pi$ \\
$\vdots$ \\
\hdashline \\
\hdashline
\end{tabular} & $\begin{array}{l}\pi \\
\vdots \\
\vdots\end{array}$ & 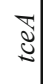 & క & $\begin{array}{l}\pi \\
0 \\
=\end{array}$ & $\begin{array}{l}5 \\
\vdots \\
\vdots\end{array}$ & ป d & : & $\begin{array}{l}\pi \\
\vdots \\
= \\
=\end{array}$ & 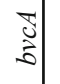 & 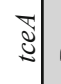 & : & \begin{tabular}{l}
$\pi$ \\
\hdashline \\
\hdashline
\end{tabular} & उ్ & ปัป & $\tilde{\Xi}$ & $\mathbb{5}$ & $\begin{array}{l}\pi \\
\vdots \\
\vdots\end{array}$ & $\stackrel{\Xi}{\Xi}$ \\
\hline & & & \multicolumn{3}{|c|}{ 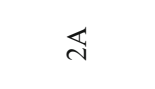 } & & \multicolumn{3}{|l|}{$\mathbb{m}$} & & \multicolumn{3}{|l|}{ 芯 } & \\
\hline
\end{tabular}




\begin{tabular}{|c|c|c|c|c|c|c|c|c|c|c|c|}
\hline \begin{tabular}{l}
0 \\
0 \\
$\pm=1$ \\
\cline { 1 - 1 }
\end{tabular} & $\begin{array}{c}0 \\
0 \\
\text { ज⿱ } \\
\\
-1\end{array}$ & 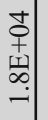 & 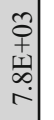 & 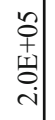 & $\begin{array}{l}\Re \\
\vdots \\
+ \\
⿱ \\
+ \\
+\end{array}$ & $\begin{array}{c} \pm \\
+ \\
+ \\
\pm n \\
\infty \\
\infty\end{array}$ & 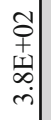 & 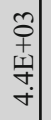 & $\begin{array}{c} \pm \\
+ \\
\pm 0 \\
\infty \\
-\end{array}$ & 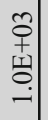 & 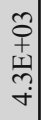 \\
\hline 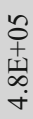 & $\begin{array}{c}+ \\
+ \\
+1 \\
\text { 离 } \\
-\end{array}$ & 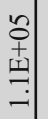 & 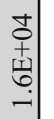 & $\begin{array}{l}8 \\
+ \\
\text { 章 } \\
.\end{array}$ & $\begin{array}{l}2 \\
0 \\
+ \\
0 \\
+ \\
\dot{+}\end{array}$ & 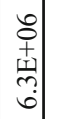 & $\begin{array}{l}r \\
0 \\
+ \\
+ \\
+ \\
\sigma \\
\sigma\end{array}$ & $\begin{array}{c}\mathbf{0} \\
+ \\
+ \\
+ \\
i\end{array}$ & 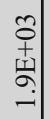 & 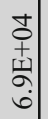 & 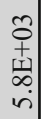 \\
\hline 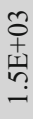 & $\begin{array}{l}8 \\
+ \\
1 \\
\text { † } \\
0\end{array}$ & $\begin{array}{c}0 \\
0 \\
+ \\
\text { ñ } \\
\\
-\end{array}$ & $\begin{array}{l}+ \\
0 \\
+ \\
\text { ț } \\
-1\end{array}$ & $\begin{array}{l}0 \\
0 \\
+ \\
\pm \\
= \\
-1\end{array}$ & 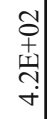 & $\begin{array}{c}+ \\
+ \\
+ \\
\pm \\
- \\
+ \\
+\end{array}$ & $\begin{array}{l}\Re \\
0 \\
1 \\
\text { ț } \\
\end{array}$ & 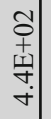 & 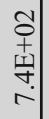 & $\begin{array}{c}\Re \\
0 \\
+ \\
= \\
= \\
=\end{array}$ & $\begin{array}{c}\tilde{0} \\
+ \\
\text { ț } \\
0 \\
\dot{r}\end{array}$ \\
\hline 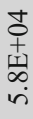 & $\begin{array}{l}8 \\
+ \\
+1 \\
0 \\
0\end{array}$ & $\begin{array}{l}+ \\
+ \\
+ \\
\text { பி } \\
\dot{\nabla}\end{array}$ & $\begin{array}{l} \pm \\
+ \\
+ \\
= \\
= \\
-1\end{array}$ & $\begin{array}{l}r \\
⿱ \\
+ \\
+ \\
+ \\
+ \\
m\end{array}$ & $\begin{array}{l}+ \\
+ \\
+ \\
\text { ț } \\
i\end{array}$ & $\begin{array}{l}0 \\
0 \\
+ \\
\pm \\
0 \\
0\end{array}$ & $\begin{array}{c}+ \\
+ \\
+ \\
\text { 音 } \\
- \\
-\end{array}$ & $\begin{array}{c} \pm \\
+ \\
\pm \\
= \\
\dot{m}\end{array}$ & $\begin{array}{c}\tilde{0} \\
+ \\
\pm \\
m \\
m\end{array}$ & $\begin{array}{c} \pm \\
+ \\
+ \\
= \\
= \\
-\end{array}$ & 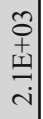 \\
\hline 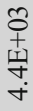 & $\begin{array}{l}8 \\
+ \\
\text { 19 } \\
0 \\
0\end{array}$ & $\begin{array}{l}0 \\
0 \\
+ \\
+ \\
+ \\
\text { in }\end{array}$ & \begin{tabular}{c}
0 \\
0 \\
+ \\
\cline { 1 - 1 } \\
$\sigma$ \\
$\sigma$
\end{tabular} & $\begin{array}{c}0 \\
+ \\
+ \\
0 \\
- \\
-\end{array}$ & 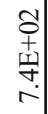 & 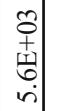 & $\begin{array}{c}0 \\
0 \\
+ \\
=1 \\
0\end{array}$ & $\begin{array}{c}0 \\
0 \\
+ \\
= \\
= \\
=\end{array}$ & 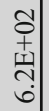 & 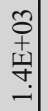 & $\begin{array}{l}0 \\
0 \\
+ \\
1 \\
\infty \\
i\end{array}$ \\
\hline 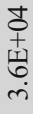 & $\begin{array}{l}8 \\
+ \\
\text { 19. } \\
0 \\
0\end{array}$ & $\begin{array}{c} \pm \\
+ \\
+ \\
\infty \\
\infty \\
\text { i }\end{array}$ & $\begin{array}{c}0 \\
0 \\
+ \\
\\
\\
\end{array}$ & 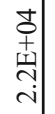 & 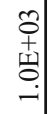 & $\mid \begin{array}{c}0 \\
0 \\
+ \\
\text { ț } \\
\dot{n}\end{array}$ & 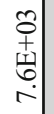 & $\begin{array}{c} \pm \\
+ \\
+ \\
\pm \\
\infty \\
\dot{m}\end{array}$ & $\begin{array}{c}0 \\
0 \\
+ \\
\text { t్d } \\
\text { - }\end{array}$ & $\begin{array}{l}\stackrel{+}{0} \\
+ \\
\stackrel{n}{N} \\
\stackrel{\sim}{*}\end{array}$ & $\begin{array}{c}\tilde{0} \\
+ \\
\stackrel{1}{m} \\
\dot{m}\end{array}$ \\
\hline $\begin{array}{c}\tilde{o} \\
+ \\
1 \\
0 \\
\dot{\gamma}\end{array}$ & $\begin{array}{l}8 \\
+ \\
+1 \\
\vdots \\
0\end{array}$ & \begin{tabular}{l}
\multicolumn{1}{c}{} \\
+ \\
\\
\\
0
\end{tabular} & 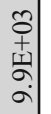 & $\begin{array}{l}0 \\
0 \\
+1 \\
1 \\
\dot{+} \\
\dot{\nabla}\end{array}$ & $\begin{array}{l}8 \\
\vdots \\
+ \\
\vdots \\
0 \\
0\end{array}$ & $\mid \begin{array}{l}0 \\
0 \\
+ \\
\text { th } \\
\text { in }\end{array}$ & $\begin{array}{c}0 \\
0 \\
1 \\
1 \\
\\
m\end{array} \mid$ & $\left|\begin{array}{c}0 \\
0 \\
1 \\
1 \\
\omega \\
\infty \\
\infty\end{array}\right|$ & $\begin{array}{l}0 \\
0 \\
1 \\
\text { ț } \\
\text { in }\end{array}$ & $\begin{array}{c}0 \\
0 \\
+ \\
\text { ț } \\
-\end{array}$ & 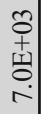 \\
\hline $\begin{array}{l} \pm \\
+ \\
+ \\
\text { m } \\
\text { in }\end{array}$ & $\begin{array}{c}8 \\
+ \\
+ \\
\text { 밈 } \\
0\end{array}$ & $\begin{array}{c} \pm \\
0 \\
+ \\
\text { ț } \\
\dot{n}\end{array}$ & $\begin{array}{c} \pm \\
0 \\
\pm \\
\text { In } \\
-\end{array}$ & $\begin{array}{c}+ \\
0 \\
\pm \\
\pm 0 \\
- \\
-1\end{array}$ & $\begin{array}{l}8 \\
+ \\
+1 \\
\text { †े } \\
\dot{0}\end{array}$ & $\begin{array}{l}+ \\
+ \\
+ \\
+ \\
+ \\
+ \\
+\end{array}$ & $\begin{array}{c}0 \\
0 \\
+ \\
100 \\
\vdots \\
\alpha\end{array}$ & 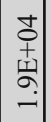 & $\begin{array}{l}m \\
+ \\
+ \\
+ \\
\dot{m} \\
m\end{array}$ & 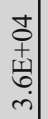 & 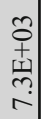 \\
\hline $\begin{array}{l}0 \\
+ \\
+ \\
\infty \\
i \\
i\end{array}$ & $\begin{array}{l}0 \\
0 \\
+ \\
+ \\
+ \\
i\end{array}$ & 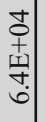 & $\begin{array}{c}+ \\
0 \\
+ \\
\text { †े } \\
- \\
-\end{array}$ & $\begin{array}{c} \pm \\
+ \\
\pm \\
\text { m } \\
-\end{array}$ & $\begin{array}{l}8 \\
\vdots \\
+ \\
\text { 됭 } \\
\dot{0}\end{array}$ & 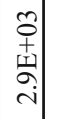 & $\begin{array}{c}0 \\
0 \\
1 \\
10 \\
0 \\
0\end{array}$ & $\mid \begin{array}{c}0 \\
+ \\
\pm \\
+1 \\
6 \\
6\end{array}$ & 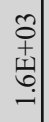 & $\begin{array}{c}\tilde{0} \\
+ \\
\omega \\
\infty \\
\infty \\
\infty\end{array}$ & $\begin{array}{c}0 \\
+ \\
1 \\
\\
\\
\dot{m}\end{array}$ \\
\hline \begin{tabular}{l} 
\pm \\
0 \\
+ \\
\multicolumn{1}{c}{} \\
i
\end{tabular} & $\begin{array}{l}0 \\
0 \\
+1 \\
=1\end{array}$ & 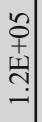 & $\begin{array}{c}+ \\
0 \\
\pm \\
= \\
\text { ì }\end{array}$ & $\begin{array}{c}+ \\
+ \\
\pm \\
m \\
\dot{+}\end{array}$ & $\begin{array}{c}8 \\
\vdots \\
\text { ज्ञ } \\
\vdots\end{array}$ & $\begin{array}{c}+ \\
0 \\
+ \\
\pm n n \\
i\end{array}$ & $\begin{array}{c}+ \\
+ \\
+ \\
\stackrel{\sim}{\sim} \\
-\end{array}$ & 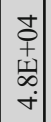 & 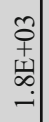 & 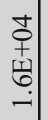 & 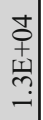 \\
\hline$\stackrel{\Xi}{\square}$ & 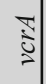 & $\begin{array}{l}\pi \\
\vdots \\
\vdots\end{array}$ & ֻْ & $\begin{array}{l}\tilde{0} \\
0 \\
0\end{array}$ & \begin{tabular}{l}
$\pi$ \\
$\vdots$ \\
$\vdots$ \\
\hdashline
\end{tabular} & $\begin{array}{c}\pi \\
\vdots \\
\vdots\end{array}$ & 胥 & $\mid \begin{array}{c}\tilde{5} \\
0 \\
0\end{array}$ & \begin{tabular}{c}
$\pi$ \\
0 \\
\hdashline
\end{tabular} & $\begin{array}{l}5 \\
\vdots \\
\vdots\end{array}$ & 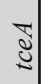 \\
\hline in & & & & 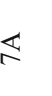 & & & & ธี & & & \\
\hline
\end{tabular}




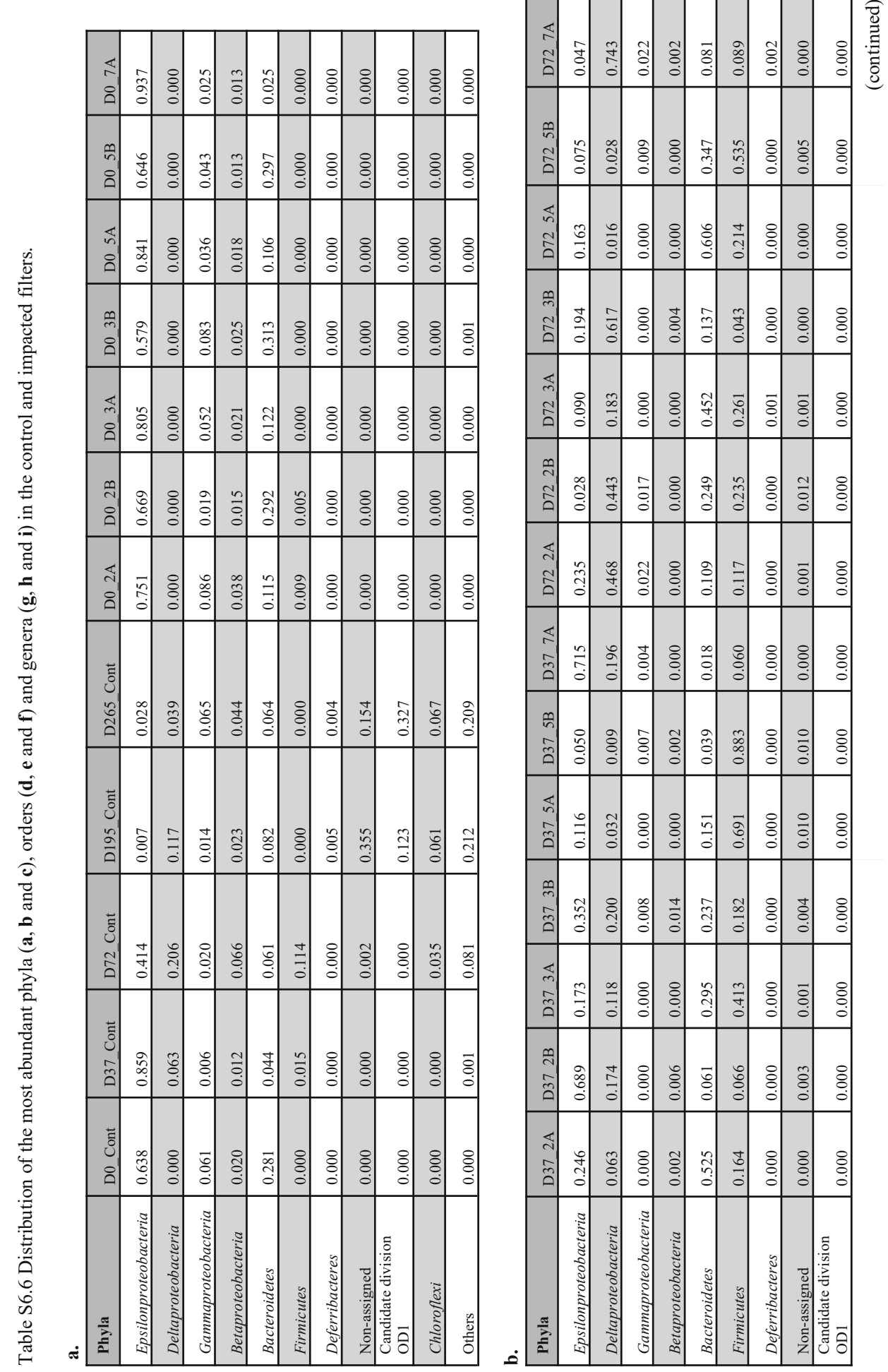




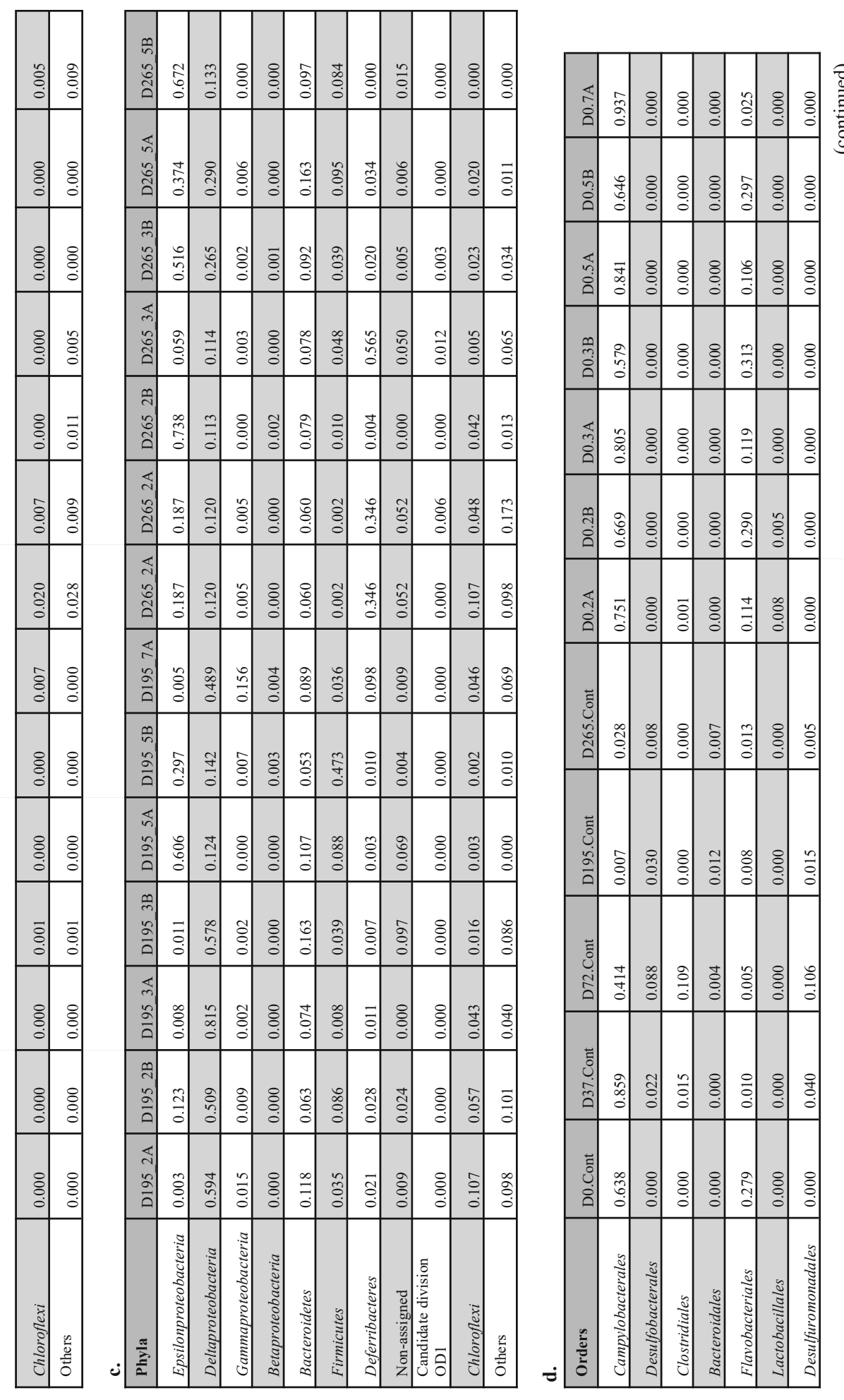




\begin{tabular}{|c|c|c|c|c|c|c|c|c|c|c|c|c|}
\hline $\begin{array}{l}: \\
: \\
:\end{array}$ & : & : & $\begin{array}{l}0 \\
\end{array}$ & : & $\begin{array}{l}0 \\
0 \\
\end{array}$ & \begin{tabular}{ll|l}
$:$ & 0 \\
\end{tabular} & : & \begin{tabular}{|l|} 
\\
\\
\end{tabular} & : & : & $\begin{array}{l}: \\
:\end{array}$ & $\begin{array}{l}0 \\
\end{array}$ \\
\hline \begin{tabular}{l|l}
$:$ & $\vdots$ \\
$:$ &
\end{tabular} & & : & $\begin{array}{l}0 \\
\vdots \\
:\end{array}$ & 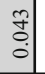 & : & \begin{tabular}{l|l}
$:$ & 0 \\
$:$ & 0
\end{tabular} & : & $\mid \begin{array}{l}\circ \\
\vdots \\
0\end{array}$ & \begin{tabular}{l|l}
0 & \\
0 & 0 \\
0 &
\end{tabular} & $\begin{array}{l}0 \\
\vdots \\
\vdots\end{array}$ & $\begin{array}{l}0 \\
\vdots \\
:\end{array}$ & $\begin{array}{l}\text { ta } \\
\vdots \\
0\end{array}$ \\
\hline \begin{tabular}{c|c}
8 & $\vdots$ \\
$:$ &
\end{tabular} & | & : & $:$ & | & : & & : & $\mid \begin{array}{l}\circ \\
\vdots \\
\circ\end{array}$ & \begin{tabular}{l|l}
$\infty$ & 0 \\
0 \\
0
\end{tabular} & \begin{tabular}{l|l|l} 
\\
$:$
\end{tabular} & : & : \\
\hline \begin{tabular}{l|l}
$:$ & $\vdots$ \\
$:$ & \\
\end{tabular} & : & : & $\begin{array}{l}\vdots \\
\vdots \\
0\end{array}$ & 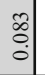 & : & & : & $\mid \begin{array}{l}\circ \\
\vdots \\
:\end{array}$ & : & 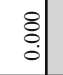 & : & : \\
\hline 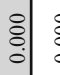 & : & : & $:$ & है. & : & \begin{tabular}{l|l|l} 
& \\
$:$ & 0
\end{tabular} & : & $\mid \begin{array}{l}\circ \\
\vdots \\
\circ\end{array}$ & :े & : & : & 吾 \\
\hline $\begin{array}{l}: \\
:\end{array}$ & : & : & $:$ & : & $:$ & \begin{tabular}{l|l|l}
$:$ & $\vdots$ \\
$:$
\end{tabular} & : & \begin{tabular}{|c|} 
\\
$\vdots$ \\
$\circ$
\end{tabular} & : & 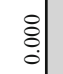 & $:$ & 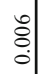 \\
\hline & & : & $\begin{array}{l}0 \\
\vdots \\
:\end{array}$ & | & : & $\begin{array}{l}: \\
\vdots \\
:\end{array}$ & 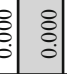 & \begin{tabular}{|c|} 
\\
\\
$\vdots$
\end{tabular} & त्] & : & : & \\
\hline & & 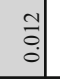 & 잉 & : & : & : & 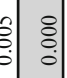 & $\mid \begin{array}{l}\bar{b} \\
\vdots \\
:\end{array}$ & : & . & : & तี \\
\hline & 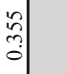 & : & తa & : & $:$ & : & : & : & : & 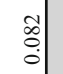 & 年 & \ุำ \\
\hline & $\begin{array}{l} \\
\vdots \\
\vdots \\
\end{array}$ & 蒗 & : & | & : & 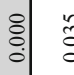 & 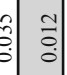 & \begin{tabular}{|c|}
$\circ$ \\
$\vdots$ \\
$\vdots$
\end{tabular} & : & : & s. & 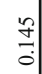 \\
\hline & & . & : & : & : & \begin{tabular}{l|l|l}
$:$ & \\
$:$ & 0
\end{tabular} & $:$ & : & |. & : & : & $\stackrel{m}{3}$ \\
\hline & & : & 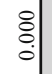 & | & 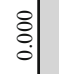 & . & : & 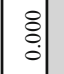 & $\begin{array}{l}\infty \\
\vdots \\
0\end{array}$ & $\begin{array}{l}0 \\
\vdots \\
\vdots \\
\end{array}$ & : & ๕̊ \\
\hline & & 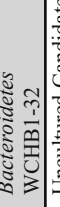 & & | & 竎 & : & : & : & 童 & 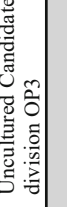 & 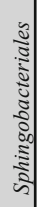 & \\
\hline
\end{tabular}

\begin{tabular}{|c|c|c|c|c|c|c|c|c|}
\hline 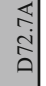 & 䗆 & & 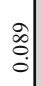 & : & : & . & & | \\
\hline & : & 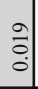 & $\stackrel{0}{0}$ & | & 일. & fol & 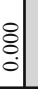 & 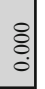 \\
\hline & 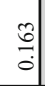 & a. & 。ٕ. & & 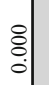 & $\frac{7}{0}$ & : & : \\
\hline & 売 & : & 8 & & | & $\mid$ & $\bar{m}$ & : \\
\hline & : & 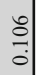 & ] & & 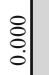 & | & of. & : \\
\hline 0 & 总 & శ్ల & तl & & | & 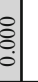 & 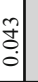 & : \\
\hline & ?ึ: & f & $\exists$ & & 䒯 & 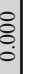 & : & : \\
\hline$\theta$ & $\begin{array}{l}n \\
0 \\
0\end{array}$ & $\stackrel{?}{?}$ & : & & | & : & $\stackrel{\circ}{\circ}$ & : \\
\hline 0 & : & $\stackrel{t}{8}$ & तु & & |̇: & . & $\tilde{g}$ & : \\
\hline ڤิ & & $\stackrel{\square}{.}$ & :ุ) & & 资 & ?] & $\stackrel{ \pm}{\partial}$ & : \\
\hline$\hat{\theta}$ & & 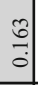 & - & & | & 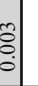 & & : \\
\hline$\ddot{\theta}$ & - & 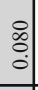 & g & & 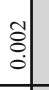 & 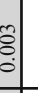 & 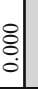 & 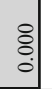 \\
\hline 0 & $\begin{array}{l}0 \\
0 \\
0 \\
0\end{array}$ & 3 & 管 & & 竞: & : & 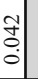 & : \\
\hline อ. & 4 & -7 & 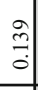 & & 靑 & 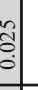 & & : \\
\hline b & ت & & & $\varpi$ & 祉 & 7 & & : \\
\hline
\end{tabular}




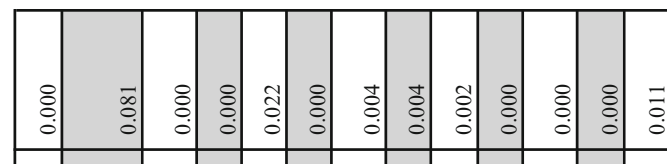

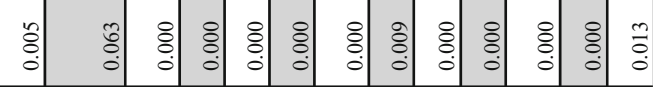

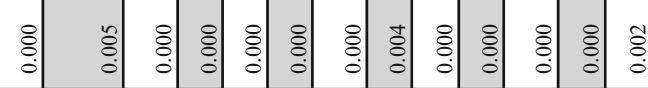

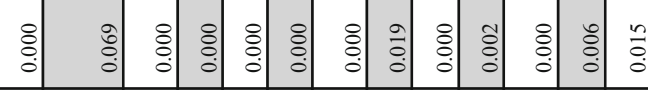

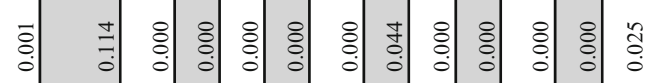

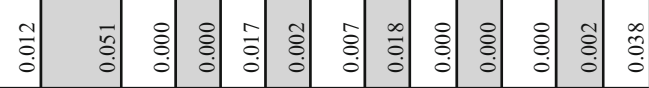

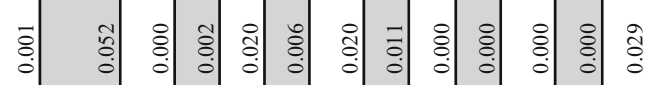

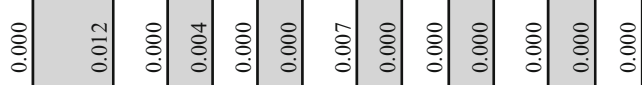

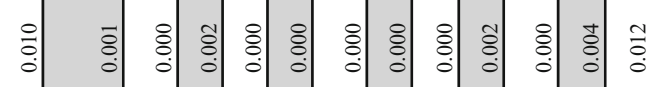

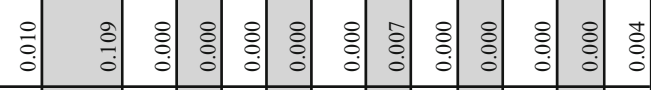

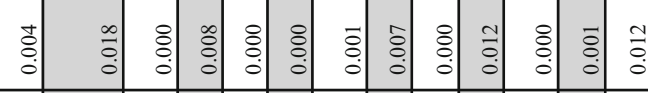

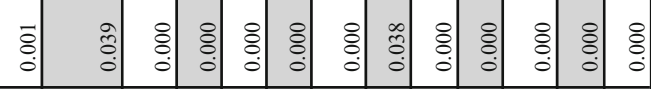

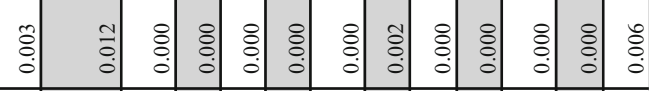

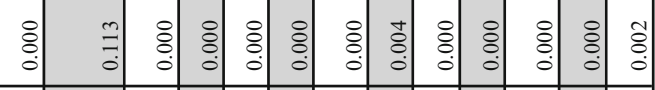

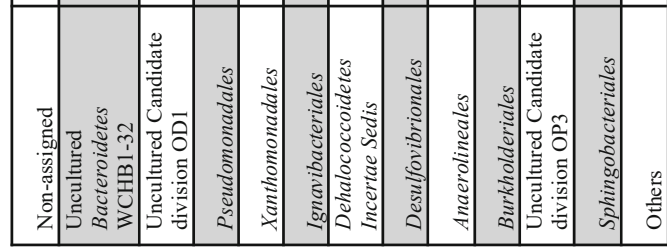

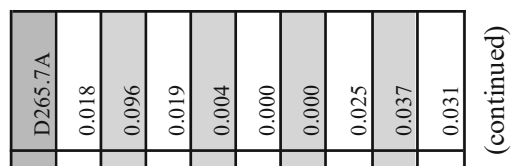

竞

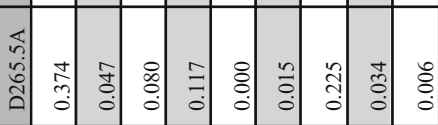

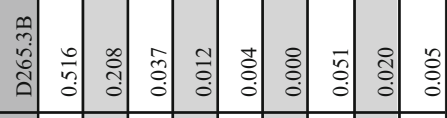

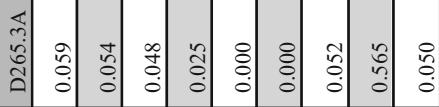

第党

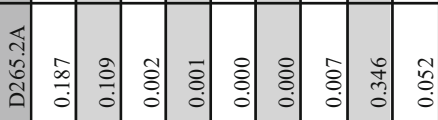

势

管

管

前

势

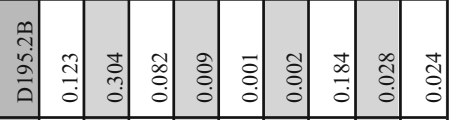

势

部

\begin{tabular}{|c|c|c|c|c|c|c|c|c|c|}
\hline 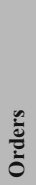 & 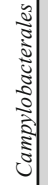 & $\begin{array}{l}0 \\
0 \\
0 \\
0 \\
0 \\
0 \\
0 \\
0 \\
0 \\
0\end{array}$ & $\begin{array}{l}0 \\
0 \\
: 3 \\
0 \\
0 \\
0 \\
0\end{array}$ & 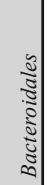 & 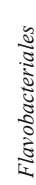 & 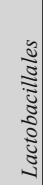 & 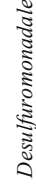 & "ै & $\begin{array}{l}\bar{\Xi} \\
0 \\
0 \\
0 \\
0 \\
i \\
\tilde{z} \\
z\end{array}$ \\
\hline
\end{tabular}




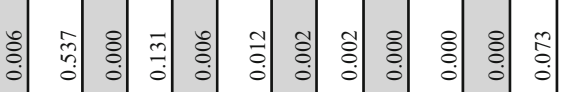

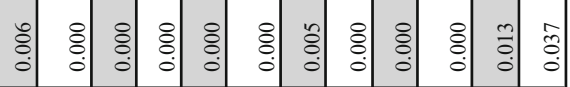

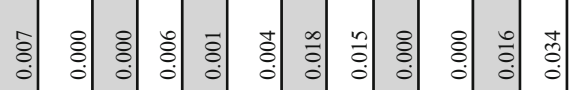

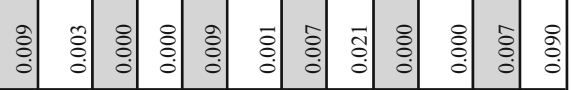

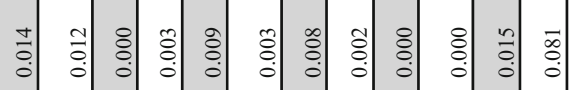

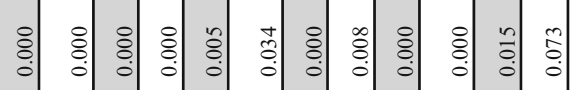

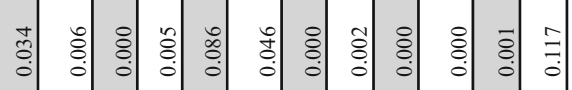

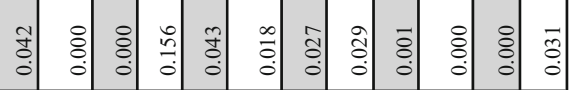

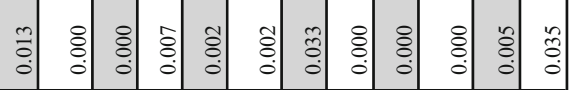

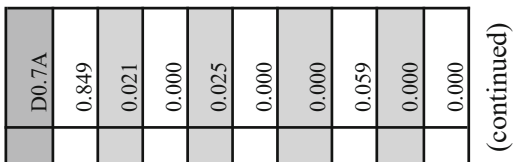

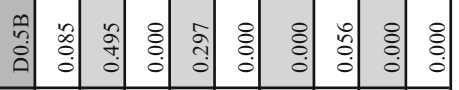

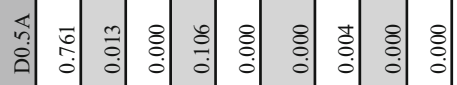

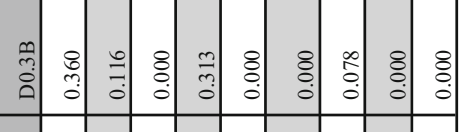

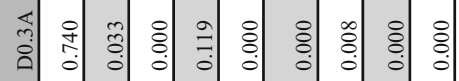

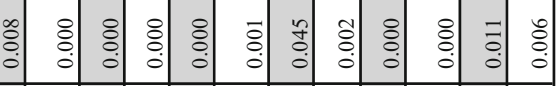

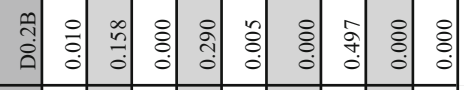

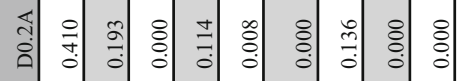

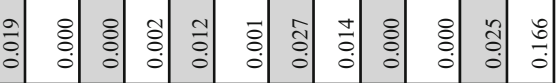

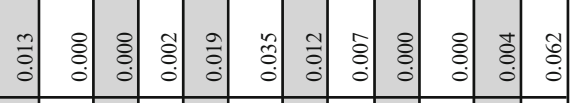

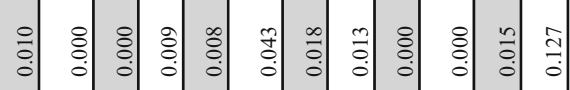

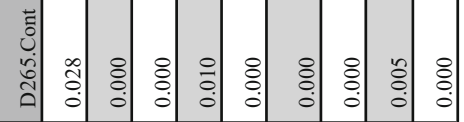

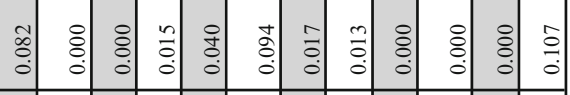

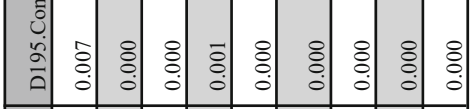

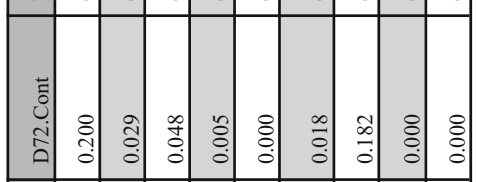

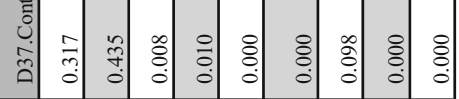

\begin{tabular}{|c|c|c|c|c|c|c|c|c|}
\hline 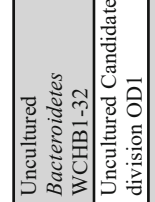 & 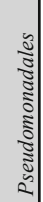 & 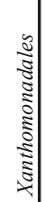 & 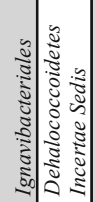 & 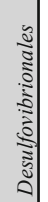 & 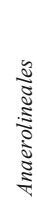 & 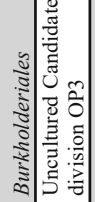 & 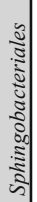 & 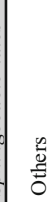 \\
\hline
\end{tabular}

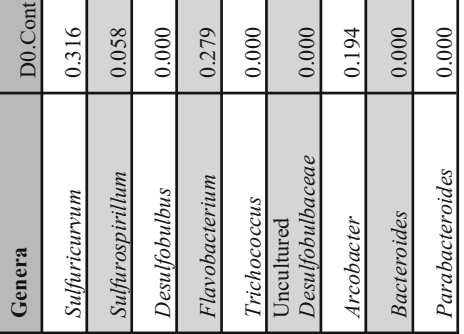




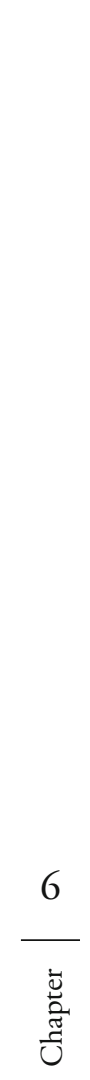

:

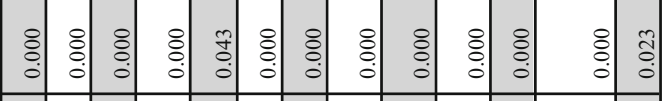
: 훙 :

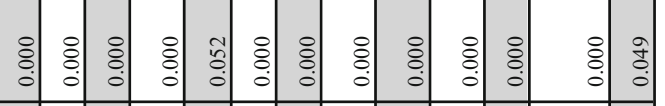
흥 홍 홍

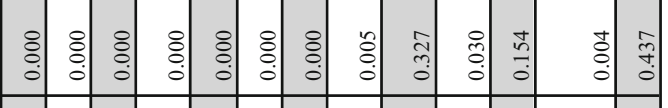

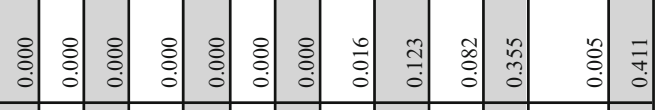

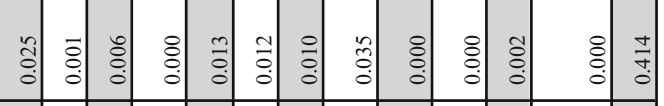

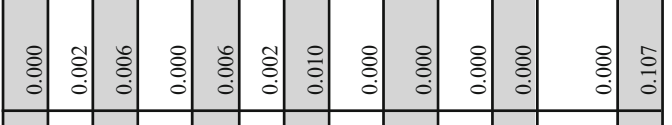
:

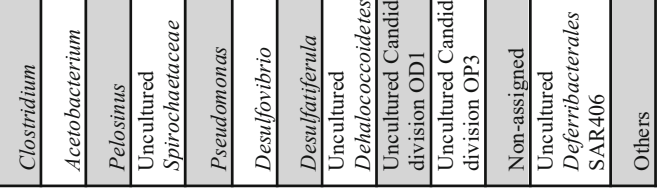

\section{拿}

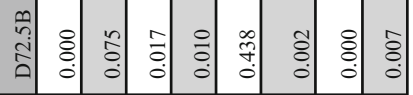

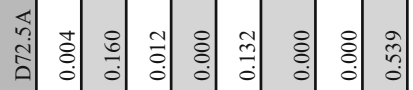

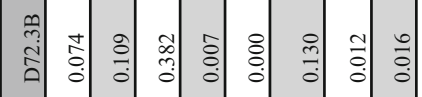

诸

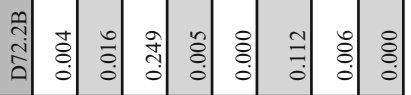

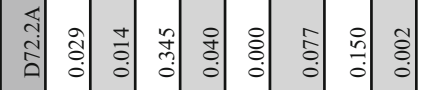

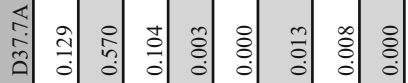

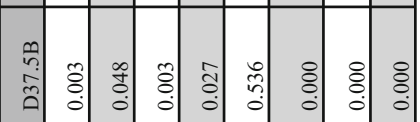

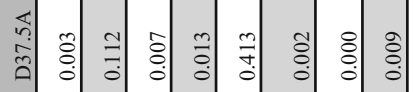

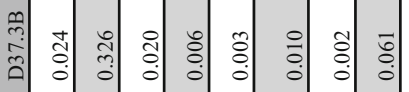

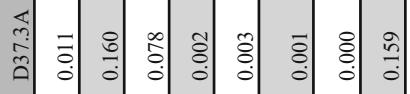

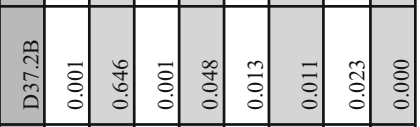

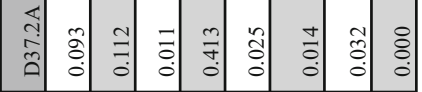

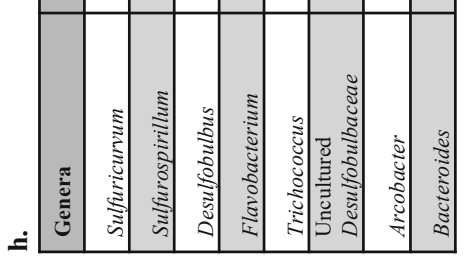




\begin{tabular}{|c|c|c|c|c|c|c|c|c|c|c|c|c|c|c|c|c|c|c|c|c|c|c|}
\hline : & $\begin{array}{l}\overrightarrow{0} \\
0 \\
\end{array}$ & : & $\begin{array}{l}\text { : } \\
\\
\end{array}$ & : & : & $\begin{array}{l}\text { ta } \\
\vdots \\
0\end{array}$ & : & $\stackrel{+}{8}$ & ఏ. & $\begin{array}{l}0 \\
\vdots \\
0 \\
\end{array}$ & $\begin{array}{l}0 \\
\vdots \\
0 \\
\end{array}$ & $\begin{array}{l}\tilde{\delta} \\
\vdots \\
\vdots\end{array}$ & $\begin{array}{l}0 \\
5 \\
0\end{array}$ & & & $\begin{array}{l}\infty \\
\stackrel{0}{0} \\
\circ\end{array}$ & $\begin{array}{l}\text { : } \\
\text {. } \\
\end{array}$ & $\begin{array}{l}0 \\
0 \\
0 \\
0\end{array}$ & ¿े़ी & 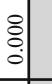 & $\begin{array}{l}\text { 이 } \\
0 \\
0\end{array}$ & \\
\hline 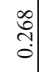 & $\begin{array}{l}+ \\
\vdots \\
0\end{array}$ & : & శ్ & $\stackrel{8}{\circ}$ & $\begin{array}{l}8 \\
: \\
0\end{array}$ & : & 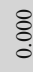 & : & : & $\begin{array}{l}8 \\
\vdots \\
0\end{array}$ & $\begin{array}{l}u \\
0 \\
0 \\
0\end{array}$ & $\begin{array}{l}8 \\
\vdots \\
0\end{array}$ & $\begin{array}{c}\stackrel{\pi}{ \pm} \\
0\end{array}$ & & & $\begin{array}{l}\infty \\
\vdots \\
\vdots \\
0\end{array}$ & 苞 & 응 & $\begin{array}{l}8 \\
\vdots \\
:\end{array}$ & $\begin{array}{l}0 \\
\vdots \\
\vdots \\
\vdots\end{array}$ & $\begin{array}{l}n \\
\vdots \\
0\end{array}$ & 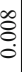 \\
\hline $\begin{array}{l}0 \\
\stackrel{2}{0} \\
\stackrel{2}{0}\end{array}$ & $\begin{array}{l}8 \\
\vdots \\
0\end{array}$ & ̊̊̊. & $\begin{array}{l}0 \\
0 \\
0 \\
0 \\
0\end{array}$ & $\stackrel{\circ}{\circ}$ & $\begin{array}{l}\text { \& } \\
\\
\end{array}$ & $\begin{array}{l}\text { t: } \\
\\
0\end{array}$ & ๑̊ి. & @: & $\stackrel{8}{\circ}$ & $\begin{array}{l}\circ \\
\vdots \\
:\end{array}$ & $\begin{array}{l}8 \\
\vdots \\
0\end{array}$ & $\begin{array}{l}\stackrel{8}{0} \\
0\end{array}$ & 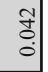 & & & 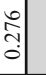 & לे. & $\begin{array}{l}0 \\
\vdots \\
0 \\
0\end{array}$ & ¿े & $\begin{array}{l}n \\
\vdots \\
0 \\
0\end{array}$ & :े & ô \\
\hline $\begin{array}{l}\text {. } \\
\text {. }\end{array}$ & $\begin{array}{l}5 \\
8 \\
0\end{array}$ & $\begin{array}{l}8 \\
\circ \\
\circ\end{array}$ & $\begin{array}{l}\text { थे } \\
0 \\
0\end{array}$ & : & $\begin{array}{l}8 \\
0 \\
0\end{array}$ & :े & ঙ্ & $\stackrel{8}{\circ}$ & ঃั & $\begin{array}{l}\stackrel{0}{0} \\
\vdots \\
\end{array}$ & $\begin{array}{l}8 \\
\vdots \\
0 \\
0\end{array}$ & $\begin{array}{l}\stackrel{\vdots}{0} \\
\vdots\end{array}$ & $\begin{array}{l}: \\
: \\
0 \\
0\end{array}$ & & & $\begin{array}{c}\text { की } \\
\stackrel{\infty}{0}\end{array}$ & $\begin{array}{l}\text { : } \\
\text { : }\end{array}$ & 勇 & $\begin{array}{l}\text { ta } \\
\vdots \\
0\end{array}$ & $\begin{array}{l}\stackrel{1}{0} \\
\vdots \\
\circ\end{array}$ & s. & هి \\
\hline$\stackrel{\infty}{0}$ & $\begin{array}{l}0 \\
\vdots \\
0\end{array}$ & $\begin{array}{l}0 \\
\dot{0}\end{array}$ & $\begin{array}{l}\vec{b} \\
: \\
\end{array}$ & $\begin{array}{l}\stackrel{0}{0} \\
\circ\end{array}$ & $\begin{array}{l}\circ \\
\vdots \\
0\end{array}$ & 苟 & \&े & : & $\stackrel{\circ}{\circ}$ & $\begin{array}{l}\stackrel{0}{0} \\
\vdots\end{array}$ & $\overrightarrow{8}$ & $\begin{array}{l}\vec{s} \\
\dot{0}\end{array}$ & 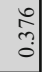 & & & है & : & ô & ¿े| & $\begin{array}{l}\text { ڤे } \\
\stackrel{0}{\circ}\end{array}$ & $\begin{array}{l}0 \\
8 \\
0 \\
0\end{array}$ & ఃे \\
\hline : & $\begin{array}{l}0 \\
\vdots \\
0\end{array}$ & $\stackrel{8}{0}$ & $\begin{array}{l}0 \\
0 \\
0 \\
0\end{array}$ & $\begin{array}{l}\stackrel{8}{0} \\
\circ\end{array}$ & ¿े & $\begin{array}{l}\infty \\
\stackrel{0}{0} \\
0\end{array}$ & $\stackrel{n}{\circ}$ & $\stackrel{\circ}{0}$ & ఏ. & $\begin{array}{l}\circ \\
\vdots \\
\circ\end{array}$ & $\begin{array}{l}7 \\
\vdots \\
0\end{array}$ & $\begin{array}{l}\vdots \\
\vdots \\
\vdots\end{array}$ & $\begin{array}{l}0 \\
\vdots \\
0 \\
0\end{array}$ & & & $\begin{array}{l}\text { oे } \\
\text { oे }\end{array}$ & $\begin{array}{l}\text { : } \\
: \\
:\end{array}$ & $\begin{array}{l}0 \\
\vdots \\
0\end{array}$ & : & $\begin{array}{l}\text { ڤे } \\
\vdots\end{array}$ & $\begin{array}{l}0 \\
0 \\
0\end{array}$ & : \\
\hline : & $\begin{array}{l}+ \\
8 \\
0\end{array}$ & $\stackrel{\circ}{\circ}$ & $\begin{array}{l}1 \\
\vdots \\
0 \\
0 \\
0\end{array}$ & $\begin{array}{l}\stackrel{\circ}{0} \\
\vdots\end{array}$ & $\begin{array}{l}\text { ô } \\
\text { : }\end{array}$ & 司 & $\stackrel{\circ}{\stackrel{0}{\circ}}$ & 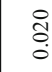 & $\stackrel{8}{\circ}$ & $\begin{array}{l}0 \\
\vdots \\
\circ\end{array}$ & $\overrightarrow{8}$ & \begin{tabular}{l}
0 \\
$\vdots$ \\
\hdashline \\
\end{tabular} & సี & & & $\stackrel{\infty}{\circ}$ & $\begin{array}{l}\text { : } \\
\vdots\end{array}$ & $\begin{array}{l}a \\
o \\
o\end{array}$ & $\begin{array}{l}\text { ¿े } \\
\vdots\end{array}$ & $\begin{array}{l}\text { ఏे } \\
\vdots\end{array}$ & तี & ڤ్ \\
\hline $\begin{array}{l}\circ \\
\stackrel{0}{0}\end{array}$ & $\begin{array}{l}m \\
\vdots \\
0\end{array}$ & छे & $\begin{array}{l}0 \\
\vdots \\
0 \\
0\end{array}$ & 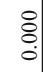 & $\begin{array}{l}+ \\
\grave{0} \\
0\end{array}$ & \begin{tabular}{l} 
: \\
$:$ \\
\hdashline
\end{tabular} & $\stackrel{8}{\circ}$ & $\stackrel{\tilde{8}}{0}$ & $\stackrel{8}{\circ}$ & $\begin{array}{l}\text { : } \\
\vdots\end{array}$ & $\begin{array}{l}8 \\
\vdots \\
0\end{array}$ & $\begin{array}{l}\stackrel{8}{\circ} \\
\stackrel{0}{0}\end{array}$ & $\begin{array}{c}0 \\
\stackrel{0}{0}\end{array}$ & & & 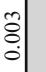 & ठี & 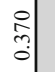 & ธี & ڤे & $\stackrel{?}{0}$ & ঃे \\
\hline$\stackrel{0}{0}$ & $\begin{array}{l}\mathbb{Z} \\
-0\end{array}$ & $\stackrel{\overbrace{}}{3}$ & $\begin{array}{l}0 \\
\vdots \\
0 \\
0\end{array}$ & $\begin{array}{l}8 \\
\vdots \\
\circ\end{array}$ & :̊ & : & ¿. & ঃे & $\stackrel{\circ}{\circ}$ & $\begin{array}{l}\vdots \\
\vdots \\
\circ\end{array}$ & $\begin{array}{l}0 \\
\vdots \\
0\end{array}$ & $\begin{array}{l}8 \\
\stackrel{0}{0}\end{array}$ & $\begin{array}{l}\text { 足 } \\
\vdots \\
0 \\
0\end{array}$ & & & $\stackrel{\infty}{-\infty}$ & : & 今̂ & $\begin{array}{l}8 \\
\stackrel{1}{0} \\
\circ\end{array}$ & 今े & : & $\frac{m}{8}$ \\
\hline$\stackrel{7}{0}$ & $\begin{array}{l}\text { ? } \\
\vdots \\
0 \\
0\end{array}$ & $\stackrel{\leftrightarrow}{0}$ & $\begin{array}{l}\vec{n} \\
0 \\
0\end{array}$ & $\begin{array}{l}\text { \&े } \\
\stackrel{0}{0}\end{array}$ & $\begin{array}{l}8 \\
: \\
:\end{array}$ & ò & @ั & $\stackrel{\circ}{\circ}$ & $\stackrel{8}{\circ}$ & $\begin{array}{l}\text { : } \\
\vdots \\
0\end{array}$ & $\begin{array}{l}\circ \\
\vdots \\
0\end{array}$ & $\begin{array}{l}\text { \&े } \\
\vdots\end{array}$ & त़े & & & ¿̊: & $\begin{array}{l}8 \\
\vdots \\
0 \\
0\end{array}$ & $\overrightarrow{0}$ & 。̊ & $\begin{array}{l}\text { t) } \\
\vdots \\
0\end{array}$ & :ै & ๕̊ \\
\hline 공 & $\begin{array}{l}- \\
\vdots \\
0 \\
0\end{array}$ & $\begin{array}{l}\text { ते } \\
\text { o }\end{array}$ & $\begin{array}{l}0 \\
0 \\
0\end{array}$ & $\begin{array}{l}\stackrel{8}{0} \\
\vdots\end{array}$ & $\begin{array}{l}0 \\
\vdots \\
0 \\
0\end{array}$ & : & $\stackrel{\substack{\mathbb{C} \\
\hdashline}}{\circ}$ & $\stackrel{\circ}{\circ}$ & $\stackrel{8}{\circ}$ & $\begin{array}{l}\circ \\
\vdots \\
\circ\end{array}$ & $\begin{array}{l}+ \\
\vdots \\
0\end{array}$ & $\begin{array}{l}8 \\
\vdots \\
0\end{array}$ & ఫ़े| & & & ڤั & \begin{tabular}{l}
$\stackrel{8}{0}$ \\
$\vdots$ \\
\hdashline
\end{tabular} & $\frac{5}{0}$ & $\begin{array}{l}\bar{s} \\
0 \\
0\end{array}$ & 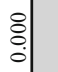 & $\begin{array}{l}\overline{\tilde{n}} \\
\text { ठn }\end{array}$ & ๑ \\
\hline a. & : & $\stackrel{-\infty}{0}$ & 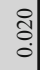 & : & : & : & : & $\stackrel{\circ}{\circ}$ & $\stackrel{\circ}{\circ}$ & : & $\overrightarrow{8}$ & 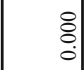 & $\begin{array}{l}n \\
0 \\
0\end{array}$ & & & ঠֶ: & $\begin{array}{l}\text { t. } \\
\text { : }\end{array}$ & ô. & : & $\begin{array}{l}: \\
\vdots\end{array}$ & ఓ⿸厃㔾. & ְ. \\
\hline : & त्ञ & $\stackrel{\circ}{\circ}$ & : & $\begin{array}{l}\text { : } \\
\circ\end{array}$ & : & : & ఏ̊. & $\stackrel{\circ}{\circ}$ & $\stackrel{8}{\circ}$ & $\begin{array}{l}\vdots \\
\vdots \\
:\end{array}$ & $\begin{array}{l}0 \\
\vdots \\
0\end{array}$ & 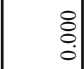 & $\frac{q}{0}$ & & & $\stackrel{7}{7}$ & $\begin{array}{l}\text { tे } \\
\text { : }\end{array}$ & $\stackrel{?}{0}$ & 형 & ठ̊. & $\stackrel{+}{0}$ & ְ. \\
\hline $\begin{array}{l}\text { : } \\
\vdots\end{array}$ & $\begin{array}{l}\tilde{s} \\
0 \\
0\end{array}$ & छे & $\begin{array}{l}n \\
0 \\
0 \\
0\end{array}$ & $\begin{array}{l}\stackrel{8}{0} \\
\circ\end{array}$ & $\begin{array}{l}8 \\
: \\
\circ\end{array}$ & : & $\begin{array}{l}8 \\
\text { हे } \\
0\end{array}$ & : & छे & $\begin{array}{l}0 \\
\vdots \\
\circ\end{array}$ & $\begin{array}{l}\text { ¿े } \\
\vdots\end{array}$ & \begin{tabular}{l} 
¿े \\
\hdashline
\end{tabular} & $\frac{0}{\grave{0}}$ & & & ๕̊. & $\begin{array}{l}\text { : } \\
\circ \\
\circ\end{array}$ & $\begin{array}{l}\infty \\
\infty \\
0\end{array}$ & $\begin{array}{l}8 \\
\vdots \\
\circ\end{array}$ & 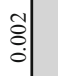 & $\stackrel{8}{\circ}$ & ¿. \\
\hline$\approx$ & 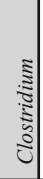 & 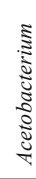 & 2. & 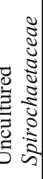 & 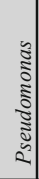 & 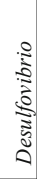 & 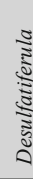 & 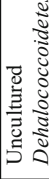 & $\begin{array}{l}\text { ิิ } \\
\text { పే }\end{array}$ & 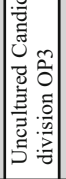 & 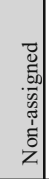 & 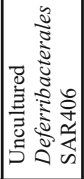 & $\begin{array}{l}\frac{2}{0} \\
\frac{0}{0} \\
\overline{0}\end{array}$ & & 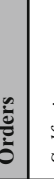 & $\bar{\Xi}$ & 竎 & 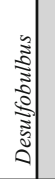 & |ี & 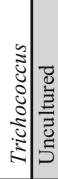 & | & \\
\hline
\end{tabular}




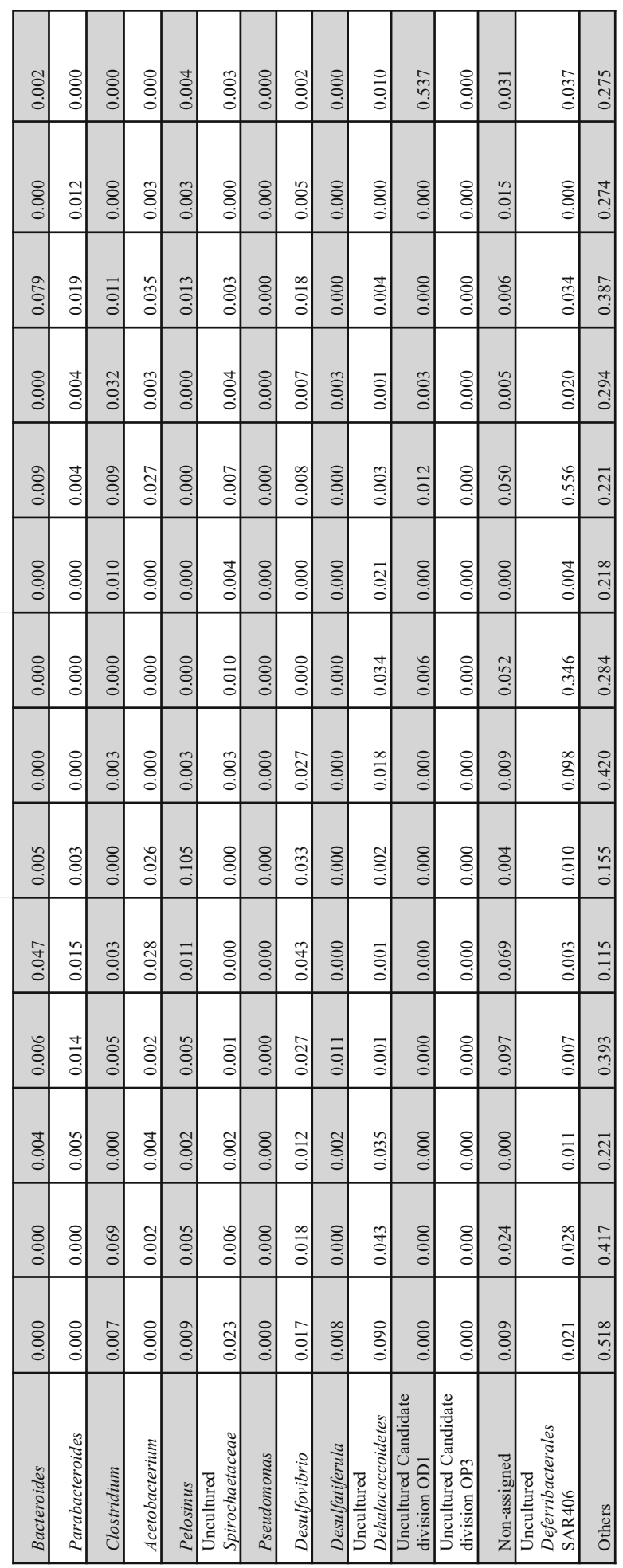


Table S6.7 Significance tests of the differences of microbial communities at order level. The groups with no significant differences $(P>0.05)$ are shown in bold.

\begin{tabular}{|l|l|l|l|l|}
\hline \multirow{2}{*}{ Sample groups } & \multicolumn{3}{l|}{ PERMANOVA $^{\mathrm{a}}$} & \multicolumn{2}{l|}{ ANOSIM $^{\mathrm{b}}$} \\
\cline { 2 - 5 } & $F$ & $P$ & $R$ & $P$ \\
\hline Control vs Day 0 & 2.0930 & 0.0090 & 0.3862 & 0.0101 \\
\hline Control vs Day 37 & 1.5131 & 0.0480 & 0.2535 & 0.0404 \\
\hline Control vs Day 72 & 2.0912 & 0.0007 & 0.5631 & 0.0013 \\
\hline Control vs Day 195 & 1.8299 & 0.0218 & 0.3991 & 0.0215 \\
\hline Control vs Day 265 & $\mathbf{1 . 1 2 8 4}$ & $\mathbf{0 . 2 3 9 7}$ & $\mathbf{0 . 1 1 5 2}$ & $\mathbf{0 . 1 3 0 1}$ \\
\hline Day 0 vs Day 37 & 3.0799 & 0.0001 & 0.5607 & 0.0006 \\
\hline Day 0 vs Day 72 & 4.8358 & 0.0004 & 0.9485 & 0.0006 \\
\hline Day 0 vs Day 195 & 4.6262 & 0.0007 & 0.8387 & 0.0006 \\
\hline Day 0 vs Day 265 & 2.9800 & 0.0001 & 0.5267 & 0.0006 \\
\hline Day 37 vs Day 72 & $\mathbf{1 . 4 5 5 1}$ & $\mathbf{0 . 0 7 1 7}$ & $\mathbf{0 . 1 6 3 3}$ & $\mathbf{0 . 0 7 9 3}$ \\
\hline Day 37 vs Day 195 & 2.0512 & 0.0200 & 0.3848 & 0.0192 \\
\hline Day 37 vs Day 265 & 1.6617 & 0.0180 & 0.2459 & 0.0146 \\
\hline Day 72 vs Day 195 & $\mathbf{1 . 3 9 8 6}$ & $\mathbf{0 . 1 1 5 2}$ & $\mathbf{0 . 1 1 1 8}$ & $\mathbf{0 . 1 1 6 6}$ \\
\hline Day 72 vs Day 265 & 2.1443 & 0.0004 & 0.4956 & 0.0006 \\
\hline Day 195 vs Day 265 & $\mathbf{1 . 5 2 9}$ & $\mathbf{0 . 0 6 5 1}$ & $\mathbf{0 . 1 6 8 1}$ & $\mathbf{0 . 0 8 1 0}$ \\
\hline
\end{tabular}

aPermutational multivariate analysis of variance using distance matrices. Significance tests were performed by $F$ test based on sequential sums of squares from permutations of the raw data.

${ }^{\mathrm{b}}$ Analysis of similarities. Statistic $R$ is based on the difference of mean ranks between groups and within groups. 
节 


\section{Organohalide respiration in pristine hypersaline lakes}

Yue Lu\#, Peng Peng\#, Alexander Martin Ruecker, Javier Ramiro-Garcia, Aleksandr Umanetc, Andreas Kappler, Detmer Sipkema, Siavash Atashgahi and Hauke Smidt. \#equal contribution 


\begin{abstract}
Despite compiling evidence for the presence of organohalide-respiring bacteria in pristine environments, there is lack of knowledge about their distribution and potential contribution to halogen cycle, especially in pristine environments with harsh $\mathrm{pH}$ and salinity such as hypersaline lakes. Biotic formation of chloromethane and chloroform was reported from Lake Strawbridge. Assuming that natural occurrence of organohalides can prime development of organohalide respiration (OHR), we prepared anoxic microcosms from Lake Strawbridge sediments. Dechlorination of amended chloroform and tetrachloroethene (PCE) to dichloromethane and trichloroethene was observed. Known chloroform and/or PCE dechlorinators were not detected in sediment microcosms and transfer cultures using MiSeq $16 \mathrm{~S}$ ribosomal RNA gene analysis and quantitative PCR, suggesting the presence of yet unknown OHRB. To our knowledge, this is the first report on OHR occurrence in extreme pristine environments. Combined with formerly documented biotic organohalogen formation, this suggests potential interdependencies between microbial chlorinators and dechlorinators in local chlorine cycle.
\end{abstract}


The long-held view that organohalides exclusively originate from anthropogenic sources was changed following the discovery of ubiquitous naturally produced organohalides [432]. Such ample production of organohalides in pristine environments can feed organohalide-respiring bacteria (OHRB) that use organohalides as electron acceptors and grow via organohalide respiration (OHR) [47, 49, 179, 433]. OHRB isolated to date have moderate traits with regards to $\mathrm{pH}$ and salinity [378], but it can be anticipated that they should also present in extreme environments such as hypersaline lakes that have been reported to emit $\mathrm{C} 1 / \mathrm{C} 2$ organohalides including but not restricted to chloromethane [39], chloroform [39, 434, 435], and tetrachloroethene (PCE) [434]. Nevertheless, to our knowledge, there is no report on the occurrence of OHRB in such extreme environments where these organisms could potentially contribute to local halogen cycles. The aim of this study was to assess OHR potential of hypersaline lake sediments and to identify the responsible OHRB.

We sampled duplicate sediment cores from two hypersaline lakes in Western Australia, i.e. Lake Strawbridge and Lake Whurr (Figure S7.1). Biotic formation of chloroform and chloromethane was previously reported from Lake Strawbridge [39], but not from Lake Whurr. Sediment cores were cut into two parts, i.e. the top layer $(0-12 \mathrm{~cm})$ and bottom layer $(>12 \mathrm{~cm})$. Geochemical properties such as salinity, total organic carbon (TOC), and $\mathrm{pH}$ were different between the two layers and between the samples of different lakes (Table 7.1). A detailed description of materials and methods used in this study is given in Supplementary Materials.

Microcosms were prepared using sediments from the two sampled lakes in two different anoxic media i.e. MGM and DBCM2 (Table S7.1, Dyall-Smith [436]). These media were previously used for the isolation of haloarchaea [436] and were selected based on our keen interest to investigate whether archaea can mediate OHR in these extreme environments. Sediment microcosms were prepared at the same salinity and $\mathrm{pH}$ as measured in the corresponding sediment layer used as inocula (Table S7.1). Chloroform or PCE were added at $1.25 \mu \mathrm{mol}$ to each bottle. Chloromethane was not used in this study due to its lower oxidation state and hence poor capacity as an electron acceptor for OHR. Chloroform was dechlorinated to dichloromethane in all microcosms derived from Lake Strawbridge sediments (Figure S7.2) but not in microcosms obtained from Lake Whurr sediments (data not shown), which is in line with the lack of natural $\mathrm{OH}$ production in the latter lake. Dechlorination of chloroform was faster in microcosms derived from bottom as compared to top layer sediments (Figure S7.2), the former being characterized by lower TOC and salinity and higher $\mathrm{pH}$ (Table 7.1). 
Furthermore, dechlorination was faster in incubations using MGM than DBCM2 medium, with the former containing peptone and yeast extract but no separate addition of trace elements and vitamins. To our surprise, PCE was dechlorinated to trichloroethene (TCE) after a long lag phase in microcosms prepared from Lake Strawbridge bottom layer sediment, but only in DBCM2 medium (Figure S7.2). It is possible that PCE was naturally formed in Lake Strawbridge but not detected by Rücker [39], or that OHRB carrying reductive dehalogenases with a broad substrate spectrum were present in the sediment and adapted to use PCE as the electron acceptor after incubation in the lab.

The microcosms derived from Lake Strawbridge bottom layer sediments and cultivated in MGM media were selected for further enrichment using chloroform. Dechlorination activity was stably maintained during three sequential transfers with dichloromethane as the end product (Figure 7.1). No dechlorination was observed in abiotic controls (data not shown) indicating OHR-mediated dechlorination.

Higher abundance of bacteria than archaea was detected in original microcosms and transfers (Figure S7.3). The archaeal abundance was decreased considerably by the third transfer, which makes their involvement in OHR unlikely. 16S ribosomal RNA (rRNA) genes of canonical chloroform- and/or PCE-dechlorinating OHRB such as Desulfitobacterium, Dehalobacter, Dehalococcoides mccartyi, Geobacter and Sulfurospirillum were not detected in any of the anoxic microcosms and transfers derived from Lake Strawbridge sediments (data not shown) using genus-specific qPCRs (Table S7.2). Principal coordinate analyses (PcoA) of 16S rRNA gene-based community patterns (MiSeq) revealed separate clustering of original sediment samples of Lake Whurr from samples of Lake Strawbridge (Figure S7.4). Besides, the initial sediment-derived microcosms and transfer cultures grouped together but distinctly separated from the original lake sediment samples, indicating their divergence from the original sediments used as inocula and convergence to a stable bacterial community during transfers (Figure S7.4). Twenty-eight of the 47 operational taxonomy units (OTUs) with relative abundance of above $2.5 \%$ in at least one sample showed higher relative abundance in enrichments and transfers compared to the original sediments inocula (Figure S7.5, Table S7.3). Twenty-four OTUs (mainly halophilic Firmicutes, Proteobacteria and Bacteroidetes) were not detected in sediment inocula (Table S7.3). Three of these OTUs were assigned to the genus of Desulfovibrio (Figure S7.6, Table S7.3). Some members of this genus are known halophenol-respirers such as Desulfovibrio sp. TBP-1 [137] and D. dechloracetivorans SF3 [138], but their ability to dechlorinate chloroform and PCE have not been reported. 
Furthermore, OTU719, assigned as uncultured Clostridiales, was the most enriched OTU in both LS1 and LS2 transfers (Figure S7.5, Table S7.3), indicating that this novel Clostridiales phylotype likely is involved in chloroform dechlorination and/or played an important role in the chloroform-dechlorinating community.

To our knowledge, OHRB have until now only been detected/isolated from habitats with neutral or acidic $\mathrm{pH}$, but not from alkaline environments like Lake Strawbridge. Our study is also the first report suggesting the presence of $\mathrm{OHR}$ in a pristine environment with high salinity. Hypersaline environments like salt lakes/marshes are widespread in the world, and areas affected by high salt concentrations will increase in the next few decades due to global sea level rise (coastal areas) and secondary salinization in inland environments. Those hypersaline environments seem to play an important role in natural $\mathrm{OH}$ emission [434, 437439]. OHRB in salt lakes might take advantage from the products of biotic chlorination as electron acceptors. Future functional (meta)genomics, further enrichment and isolation and compound specific isotope analysis will be necessary to reveal microbial players of chlorination and dechlorination, their strategy to adapt to alkaline $\mathrm{pH}$ and high salinity, and their (presumed) respective roles and interactions within local chlorine cycles. 


\section{Acknowledgements}

We would like to thank Laura A. Hug and Pascal Weigold for expert advice and technical assistance and Christoph Tubbesing for the copyright of sediment photos. We acknowledge the China Scholarship Council for the support to Yue Lu and Peng Peng. Furthermore, Siavash Atashgahi, Detmer Sipkema and Hauke Smidt received support by a grant of BEBasic-FES funds from the Dutch Ministry of Economic Affairs. Andreas Kappler and Alexander Ruecker received funding from the research unit 763 "Natural Halogenation Processes in the Environment, Atmosphere and Soil" funded by the German Research Foundation (DFG). 


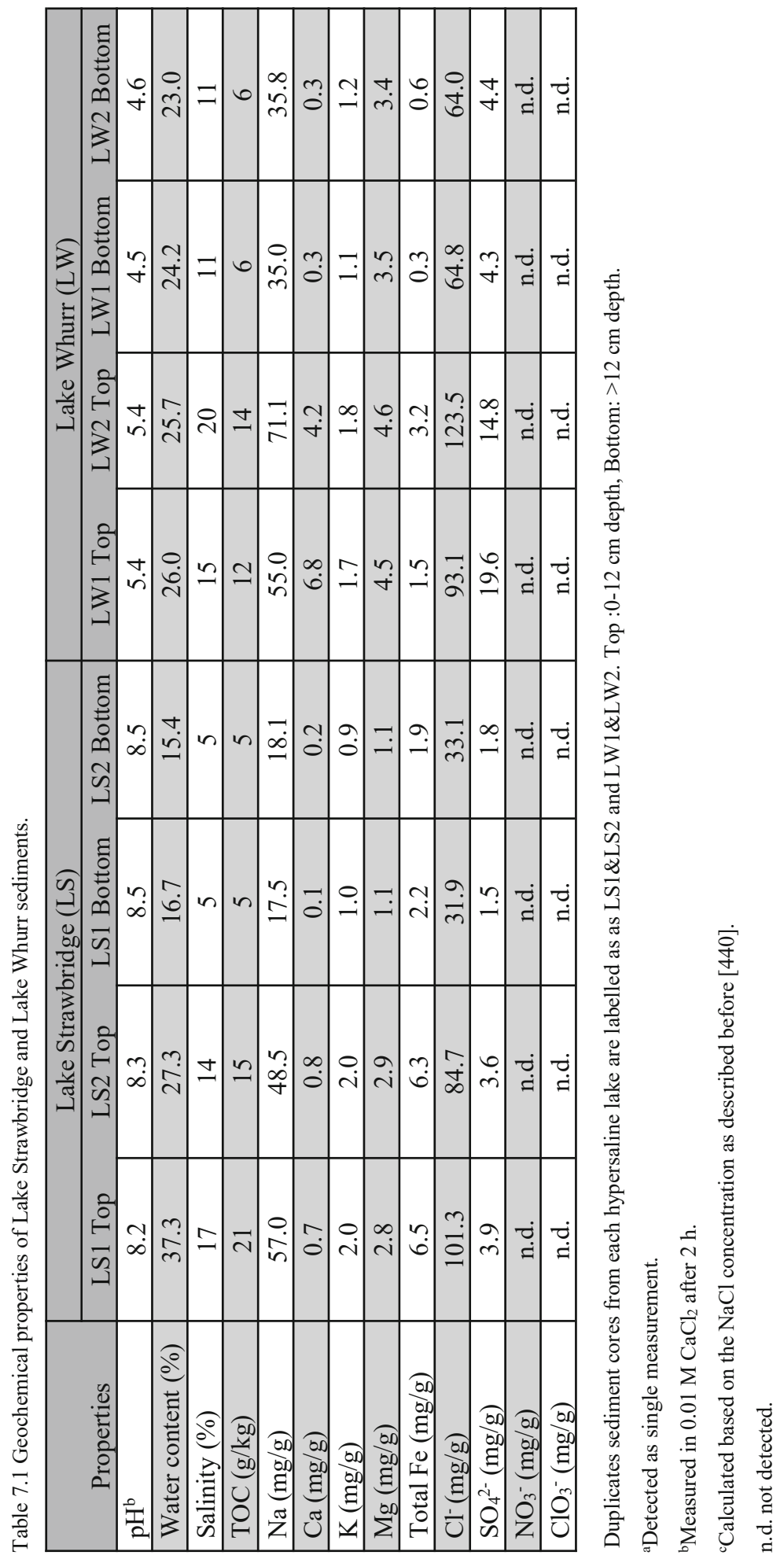


a.

$\sim$ chloroform - - dichloromethane

a.
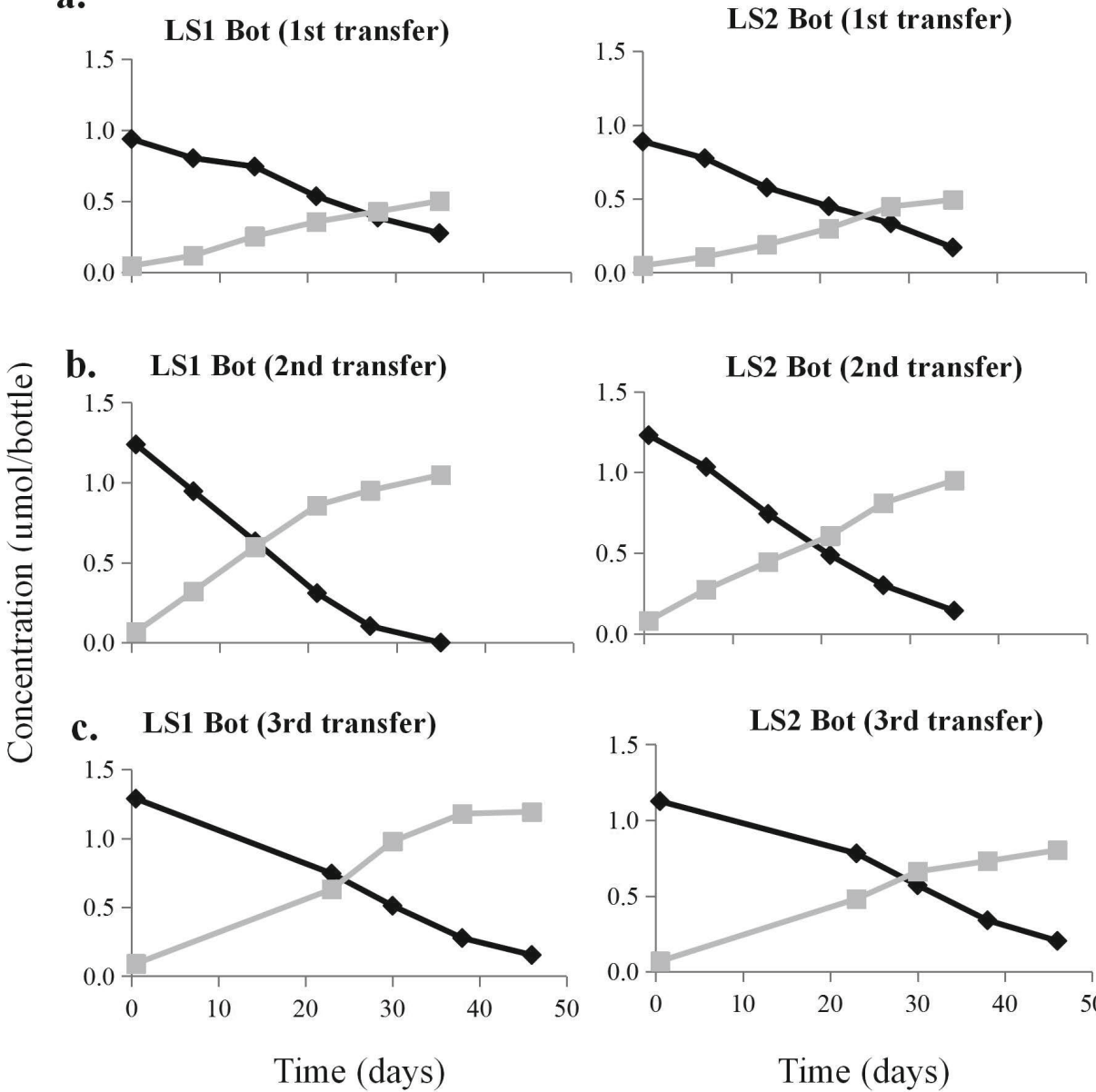

7
苛
ज्ञ

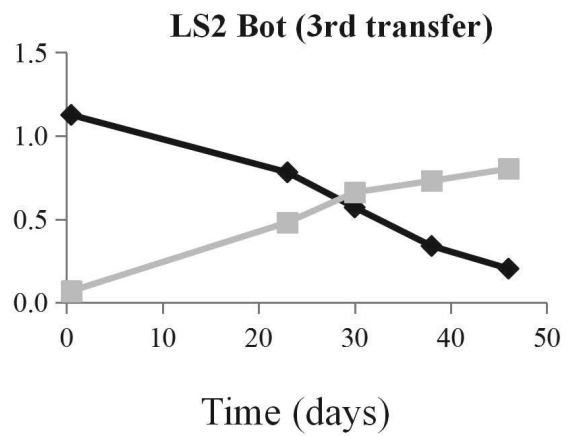

Figure 7.1 Dechlorination of chloroform in transfers of anaerobic enrichments derived from the microcosms prepared from the bottom layer (Bot, $>12 \mathrm{~cm}$ depth) sediment collected from Lake Strawbridge. Duplicates sediment cores are labelled as LS1 and LS2. a, b, c are the first, second and third transfers, respectively. 


\section{Supplementary materials}

\section{Sampling site}

Sediment samples were collected from Lake Strawbridge (LS, $\left.32.84^{\circ} \mathrm{S}, 119.40^{\circ} \mathrm{E}\right)$ and Lake Whurr (LW, $\left.33.04^{\circ} \mathrm{S}, 119.01^{\circ} \mathrm{E}\right)$, two salt lakes located in the Western Australian wheat belt (Figure S7.1). Duplicates sediment cores of approximately $24 \mathrm{~cm}$ length and $4 \mathrm{~cm}$ internal diameter were sampled from close locations within each of the two lakes and transported to the Laboratory of Microbiology, Wageningen University, Wageningen, The Netherlands. In an anoxic chamber filled with an atmosphere of $\mathrm{N}_{2} / \mathrm{H}_{2}(96 \%: 4 \%$, v/v), sediment cores were cut into two parts, i.e. the top layer $(0-12 \mathrm{~cm})$ and bottom layer $(>12 \mathrm{~cm})$. Subsamples from each sediment layer were homogenized removing big pieces of vegetation before geochemical analysis and set-up of anoxic microcosms. Aliquots of the remainder were stored in Eppendorf tubes at $-80^{\circ} \mathrm{C}$ for further molecular analysis.

\section{Geochemical analysis}

Water content was determined by weighing wet samples and subsequently drying at $105{ }^{\circ} \mathrm{C}$ overnight to a constant weight and cooling down to room temperature in a desiccator. The weight loss in percentage was calculated as the water content. $\mathrm{pH}$ was measured with air-dried sediments suspended in $0.01 \mathrm{M} \mathrm{CaCl}_{2}$ solution, and the $\mathrm{pH}$ was measured immediately and again after two hours. Sediment total organic carbon (TOC) was measured using the Kurmies method [441] in the chemical biological soil laboratory (CBLB) of Wageningen University. Low crystalline "bioavailable" iron was extracted from $0.5 \mathrm{~g}$ wet sediment for one hour in the dark using $25 \mathrm{ml}$ of $0.5 \mathrm{M}$ anoxic $\mathrm{HCl}$ [442], and concentrations of dissolved $\mathrm{Fe}(\mathrm{II})$ and $\mathrm{Fe}(\mathrm{III})$ were quantified using the spectrophotometric Ferrozine assay (Stookey, 1970). Major anions including $\mathrm{Cl}^{-}, \mathrm{SO}_{4}{ }^{2-}, \mathrm{NO}_{3}{ }^{-}$and $\mathrm{ClO}_{3}{ }^{-}$, were analyzed on a Thermo Scientific Dionex ${ }^{\mathrm{TM}}$ ICS2100 Ion Chromatography System (Dionex ICS-2100). Major cations including $\mathrm{Ca}^{2+}, \mathrm{K}^{+}$, $\mathrm{Mg}^{2+}$ and $\mathrm{Na}^{+}$were measured using inductively coupled plasma-optical emission spectroscopy (ICP-OES, Varian, The Netherlands). Salinity was calculated based on the $\mathrm{NaCl}$ concentration as described before [440].

\section{Microcosm set-up and transfers}

Hypersaline sediments from top and bottom layers of Lake Strawbridge and Lake Whurr were used for microcosm set-up. Anoxic microcosms were prepared in $50 \mathrm{ml}$ serum bottles with $2.5 \mathrm{~g}$ wet sediment and $25 \mathrm{ml}$ of either MGM or DBCM2 medium (Table S7.1, [436]. The components of the SL10 trace element solutions (per liter) were $10 \mathrm{ml}$ of $25 \% \mathrm{HCl}$; 
$\mathrm{FeCl}_{2} \cdot 4 \mathrm{H}_{2} \mathrm{O}, 1.5 \mathrm{~g} ; \mathrm{CoCl}_{2} \cdot 6 \mathrm{H}_{2} \mathrm{O}, 190 \mathrm{mg} ; \mathrm{MnCl}_{2} \cdot 4 \mathrm{H}_{2} \mathrm{O}, 100 \mathrm{mg} ; \mathrm{ZnCl}_{2}, 70 \mathrm{mg} ; \mathrm{H}_{3} \mathrm{BO}_{3}, 6 \mathrm{mg}$; $\mathrm{Na}_{2} \mathrm{MoO}_{4} \cdot 2 \mathrm{H}_{2} \mathrm{O}, 36 \mathrm{mg} ; \mathrm{NiCl}_{2} \cdot 6 \mathrm{H}_{2} \mathrm{O}, 24 \mathrm{mg} ; \mathrm{CuCl}_{2} \cdot 2 \mathrm{H}_{2} \mathrm{O}, 2 \mathrm{mg}$. The components of the Vit10 vitamin solution (per liter) were 4-aminobenzoate, $13 \mathrm{mg}$; d-(+)-biotin, $3 \mathrm{mg}$; nicotinic acid, $33 \mathrm{mg}$; hemicalcium D-(+)-pantothenate, $17 \mathrm{mg}$; pyridoxamine hydrochloride, $50 \mathrm{mg}$; thiamine chloride hydrochloride, $33 \mathrm{mg}$; cyanocobalamin, $17 \mathrm{mg}$; D,L-6,8-thioctic acid, 10 $\mathrm{mg}$; riboflavin, $10 \mathrm{mg}$; folic acid, $4 \mathrm{mg}$. Bottles were sealed with a viton stopper, and the headspace was exchanged with $\mathrm{N}_{2}$ gas $(140 \mathrm{kPa})$. Salinity and $\mathrm{pH}$ in the medium were adjusted to the same values as observed in inocula sediments (Table 7.1, Table S7.1). Chloroform or PCE were added at $1.25 \mu \mathrm{mol}$ to each bottle. A second spike $(1.25 \mu \mathrm{mol})$ of chloroform was added at day 46 to MGM medium microcosms derived from LS bottom sediments that had completely dechlorinated the first chloroform amendment. All microcosms were set up in duplicate and cultivated without shaking at $37{ }^{\circ} \mathrm{C}$ in the dark.

A $10 \%(\mathrm{v} / \mathrm{v})$ inoculum was taken from MGM medium microcosms derived from duplicate LS bottom sediments for three consecutive serial transfers using the same medium and chloroform dosing. Transfer cultures were set up as single bottles and an abiotic control was prepared by adding sterilized (autoclaving at $121{ }^{\circ} \mathrm{C}$ for $30 \mathrm{~min}$ ) inocula. All cultures were cultivated without shaking at $37^{\circ} \mathrm{C}$ in the dark.

\section{Detection of chloroform and dichloromethane}

Chlorinated methanes and ethenes were quantified by taking $0.5 \mathrm{ml}$ headspace samples of the cultures and analyzed using a gas chromatograph equipped with a flame ionization detector (GC-FID, Shimadzu 2010) as described before for chlorinated methanes [436] and chlorinated ethenes [30]. A Stabilwax column (Restek Corporation, U.S) was used with nitrogen as carrier gas at a flow of $1 \mathrm{ml} \mathrm{min} \mathrm{m}^{-1}$. A spit flow of $12 \mathrm{ml} \mathrm{min}^{-1}$ with split ratio of 1:10 was set in the injector held at $250{ }^{\circ} \mathrm{C}$. Standards were set up with the same headspace-liquid phase ratio as treatment cultures.

\section{DNA extraction}

To avoid the interference of high salinity of samples with the DNA extraction procedure [440], $0.5 \mathrm{~g}$ wet sediment was washed three times with $1.5 \mathrm{ml}$ of $10 \mathrm{mM}$ TE buffer (pH 7.0), and the washing buffer was collected and filtered through a $0.22 \mu \mathrm{m}$ Millipore membrane filter (MP, USA). DNA loss during washing was anticipated, but with limited sediment material this was the only solution found to ensure enough DNA for further analysis. Washed sediment and the Millipore membrane filter were extracted separately using the PowerSoil DNA isolation kit 
(MO-BIO, USA) following the manufacturer's instructions. DNA extracts from sediment and filter were combined for further molecular analysis as described before [443]. DNA isolation was conducted from three independent samples from each sediment layer. In total, DNA was extracted from 24 samples (two hypersaline lakes $\times$ duplicates sediment cores from each lake $\times$ two sediment layers $\times$ technical triplicates). Two $\mathrm{ml}$ of anoxic medium were sampled from anoxic microcosms and transferred cultures for DNA extraction using the same method but without the initial washing step.

\section{Illumina MiSeq sequencing and data analysis}

A 2-step PCR strategy was applied to generate barcoded amplicons from the V1-V2 region of the bacterial 16S ribosomal RNA (rRNA) genes, and the PCR products were purified and sent for sequencing on a MiSeq platform (GATC-Biotech, Konstanz, Germany) as described in chapter 5. Sequence analysis was performed with NG-Tax, an in-house pipeline [346]. Principal coordinate analyses (PcoA) plot of bacterial 16S rRNA gene-based community composition was done by using Quantitative Insights Into Microbial Ecology (QIIME) v1.2 [349]. Heatmap visualisation of microbial composition was performed using R 3.2.3 [444] and the following libraries: gplots, vegan and RColorBrewer. The Bray method implemented in the "vegan" library was used to create a dissimilarity matrix and derived dendrograms of samples and operational taxonomic units (OTUs).

\section{Phylogenetic Analysis}

Phylogenetic analysis of selected 16S rRNA gene sequences derived from the bacterial MiSeq dataset was performed via Mega 4 program [350]. The Neighbour-joining method was used to construct a phylogenetic tree.

\section{Quantitative PCR}

The abundance of 16S rRNA genes of total bacteria, archaea, and several canonical OHRB including Desulfitobacterium, Dehalobacter, Dehalococcoides mccartyi, Sulfurospirillum and Geobacter were determined by quantitative real time PCR (qPCR). Assays were performed in triplicates on a CFX384 Real-Time system in C1000 Thermal Cycler (Bio-Rad Laboratories, USA) with $\mathrm{iQ}^{\mathrm{TM}}$ SYBR Green Supermix (Bio-Rad Laboratories, USA). The primers and qPCR programs used in this study are listed in Table S7.2.

\section{Nucleotide sequences}

Nucleotide sequences were deposited in the European Nucleotide Archive (ENA) with accession number ERS1165096-ERS1165117 under study PRJEB14107. 


\section{Lake Strawbridge}

$\left(32.84^{\circ} \mathrm{S}, 119.40^{\circ} \mathrm{E}\right)$

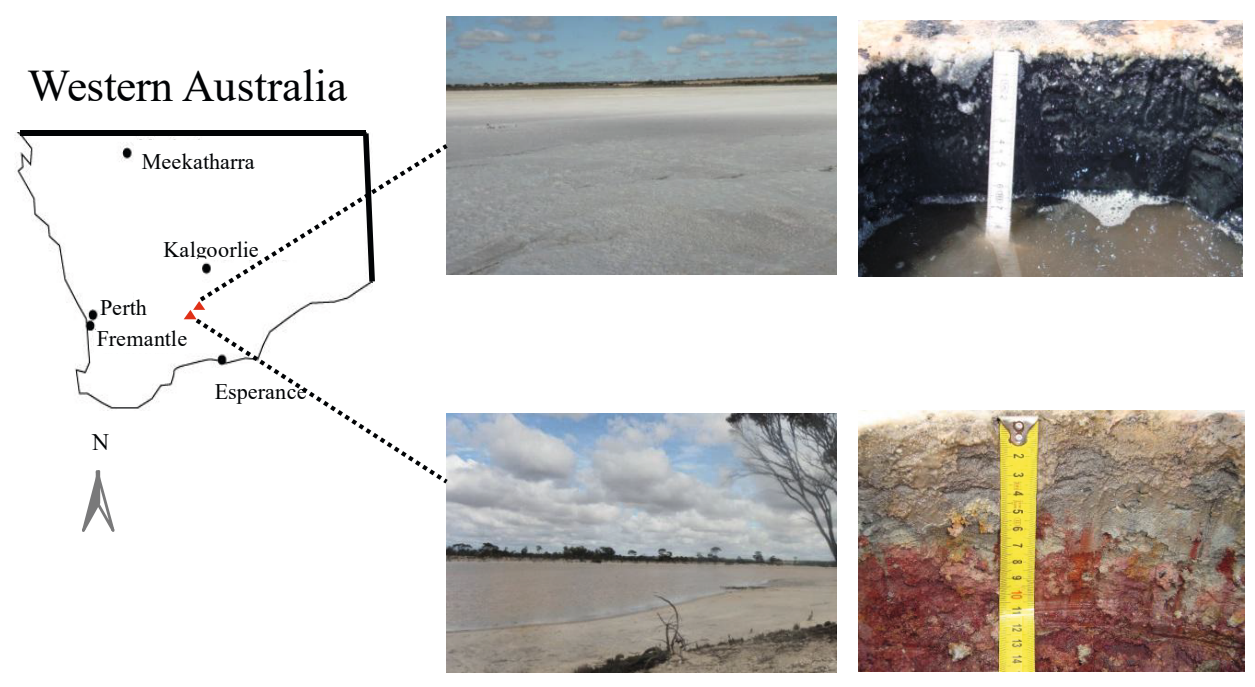

Lake Whurr

$\left(33.04^{\circ} \mathrm{S}, 119.01^{\circ} \mathrm{E}\right)$

Figure S7.1 Location of Lake Strawbridge and Lake Whurr and coordinates of the sampling points. 

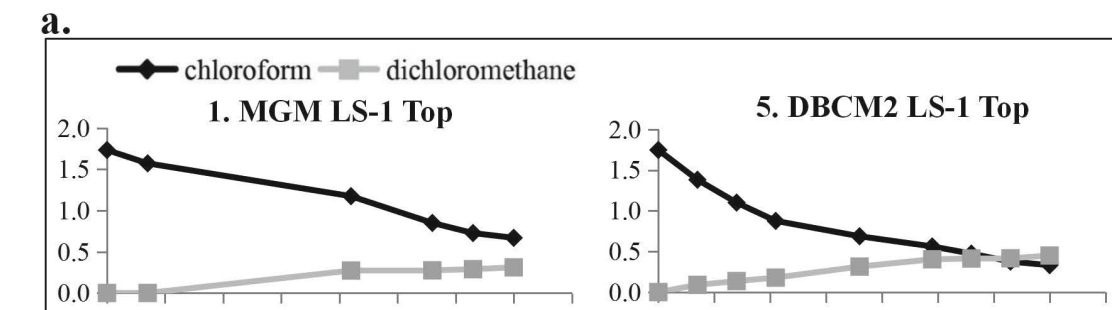

2. MGM LS-2Top

6. DBCM2 LS-2 Top
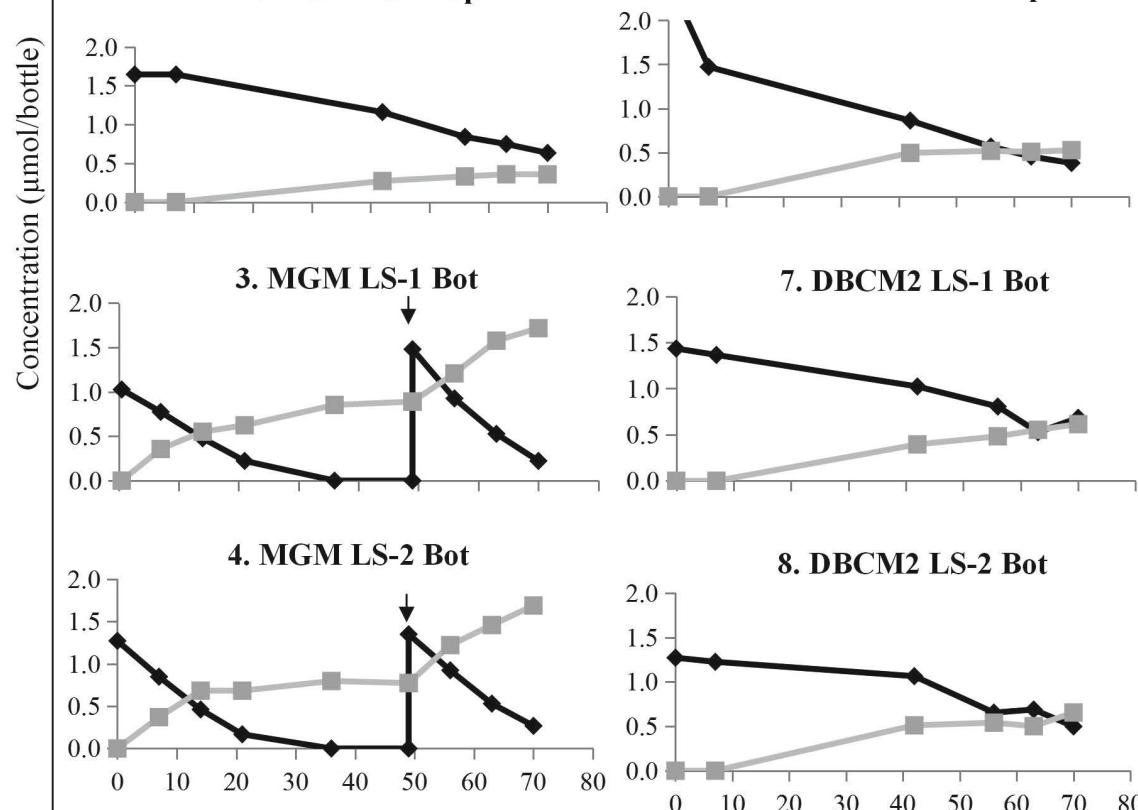

Time (days)

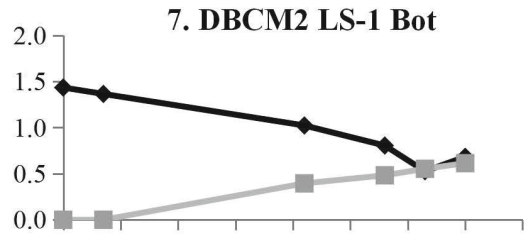

8. DBCM2 LS-2 Bot

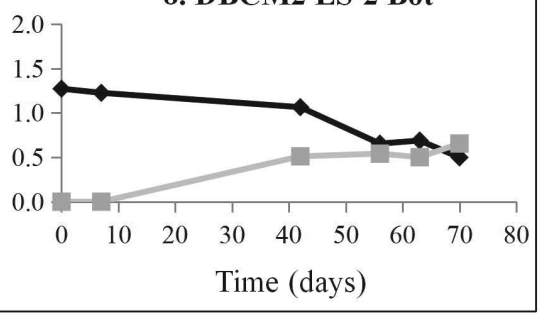

b.

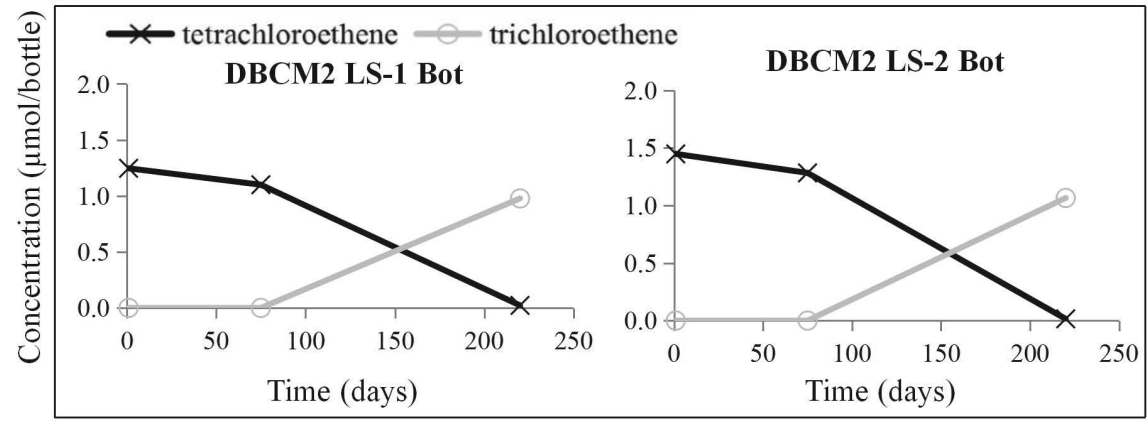

Figure S7.2 Dechlorination of chloroform (a) and tetrachloroethene (b, no analysis between day 75 and 220) in microcosms derived from the Lake Strawbridge (LS) sediment and cultivated in MGM (a1-a4) or DBCM2 (a5a8, b1-b2) media. Abbreviation: Top, top layer (0-12 cm dpth); Bot, bottom layer ( $>12 \mathrm{~cm}$ depth). Duplicates sediment cores are labelled as LS1 and LS2. Addition of second spike of chloroform is indicated by arrows. 


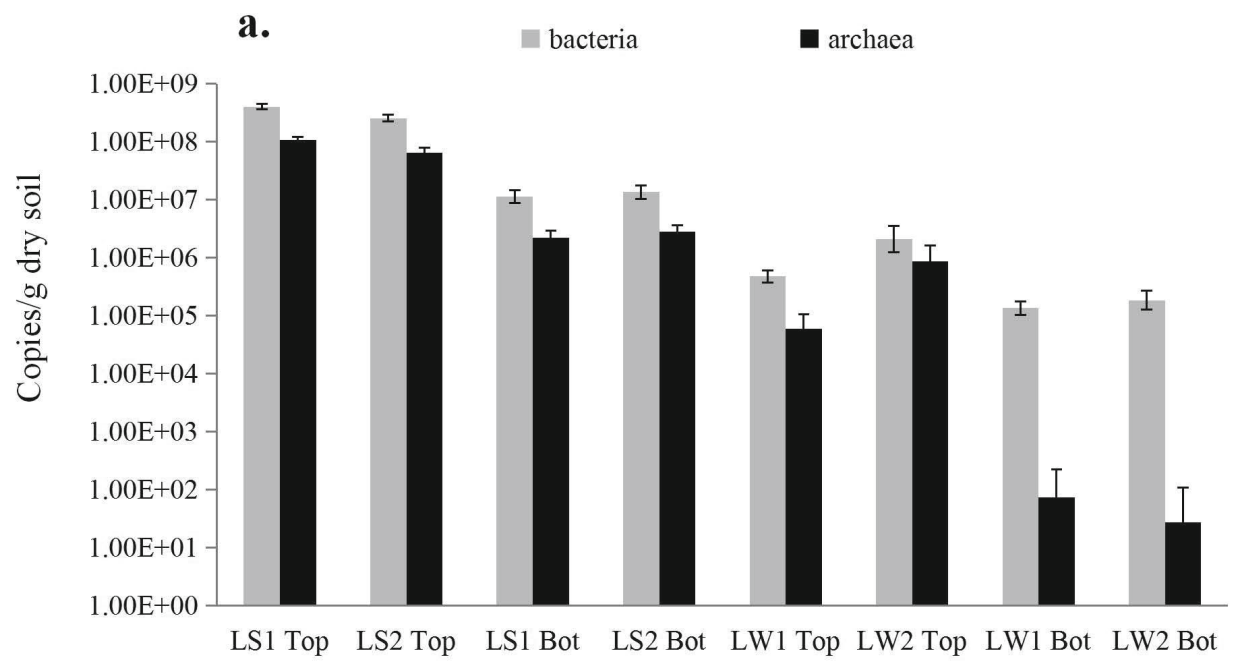

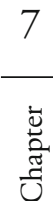

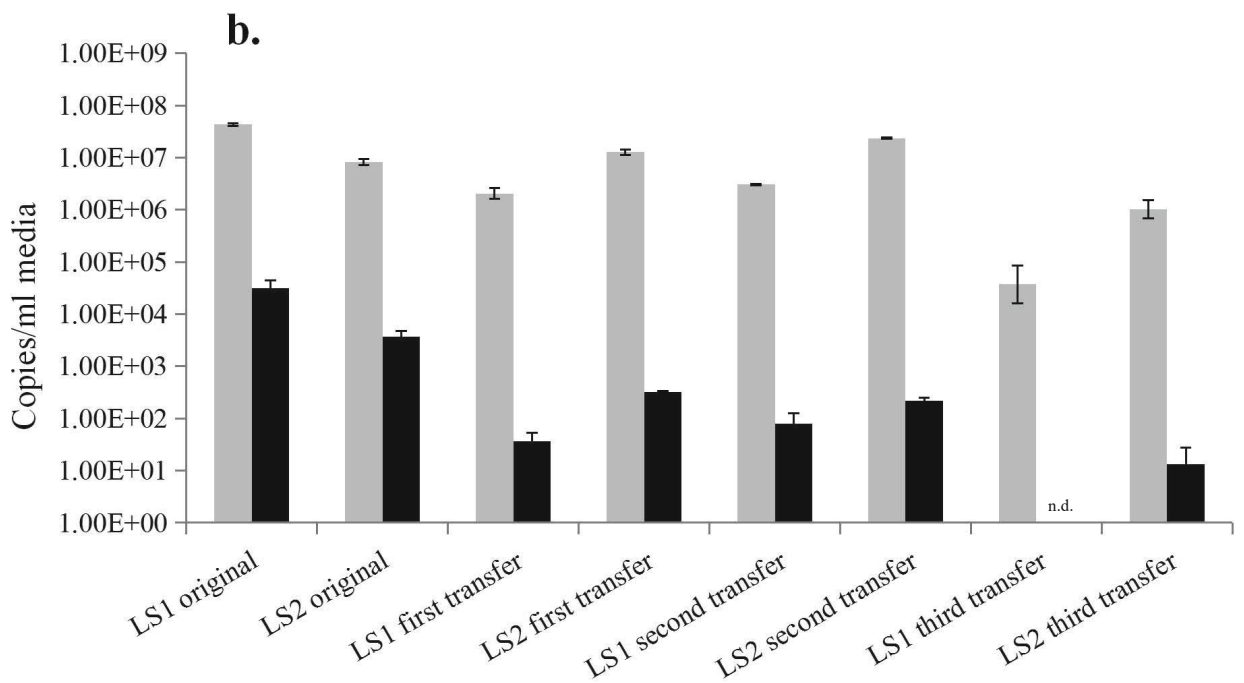

Figure S7.3 Quantitative PCR (qPCR) targeting total bacterial and archaeal 16S rRNA genes in hypersaline lake sediments (a) and during transfers (b). Abbreviation: LS, Lake Strawbridge; LW, Lake Whurr; Top, top layer (0$12 \mathrm{~cm}$ depth); Bot, bottom layer (>12 cm depth). Duplicate sediment cores from each lake are labelled as LS1\&LS2 and LW1\&LW2. Error bars in: a. represent standard deviations of three independent DNA extractions, and qPCR technical triplicates are conducted for each DNA sample and $\mathbf{b}$. standard deviations of qPCR technical triplicates from the same DNA. n.d. nondetectable. 
a.

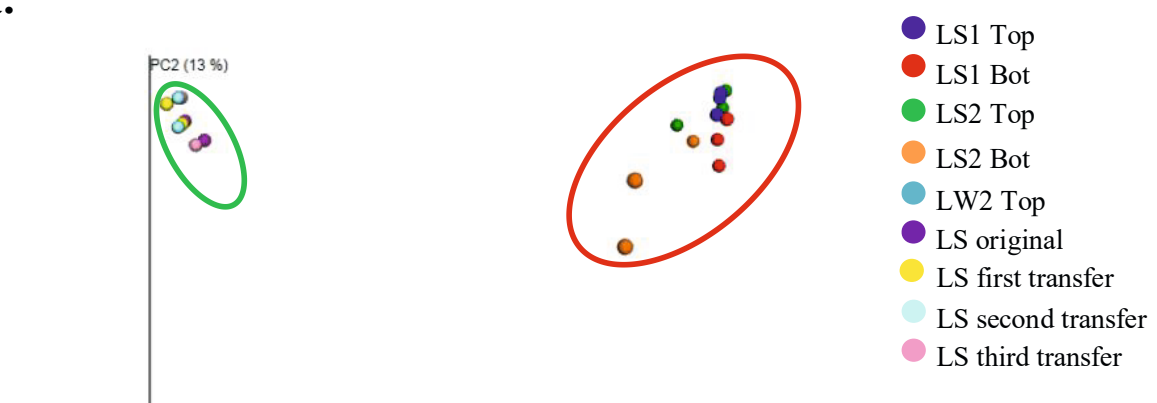

b.

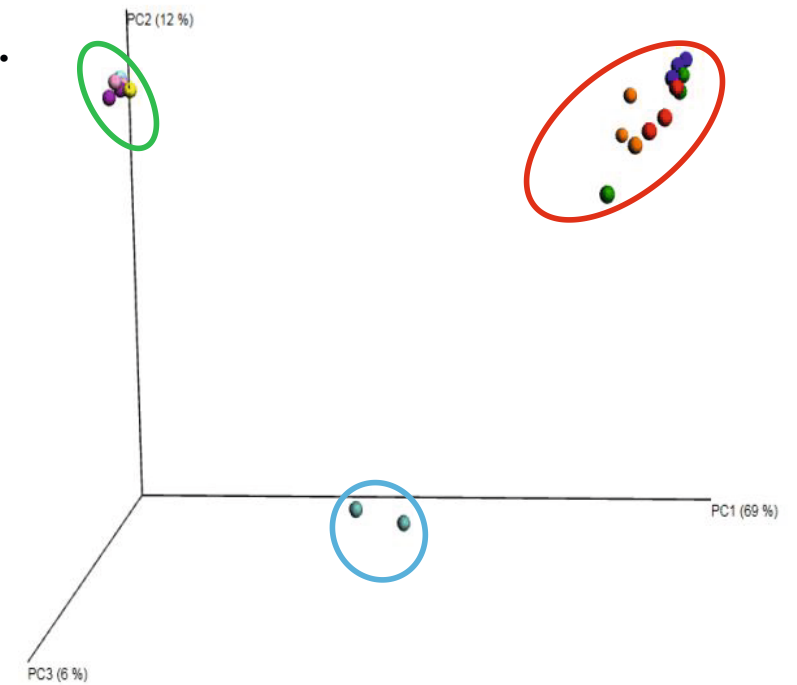

Figure S7.4 Unweighted (a) and weighted (b) principal coordinate analyses (PcoA) plot of bacterial OTUs in hypersaline lake sediments and anaerobic enrichments derived from the bottom layer sediments in Lake Strawbridge. Abbreviation: LS, Lake Strawbridge; LW, Lake Whurr; Top, top layer (0-12 cm depth); Bot, bottom layer $(>12 \mathrm{~cm}$ depth). Duplicate sediment cores from each lake are labelled as LS1\&LS2 and LW1\&LW2. No data from LW1 samples are available because of low DNA yield. Original sediment samples from Lake Whurr and Lake Strawbridge are shown within solid blue and red ovals, respectively. Anaerobic enrichments derived from bottom layer sediments in Lake Strawbridge and transfers are shown within solid green ovals. 
Relative abundance (\%)
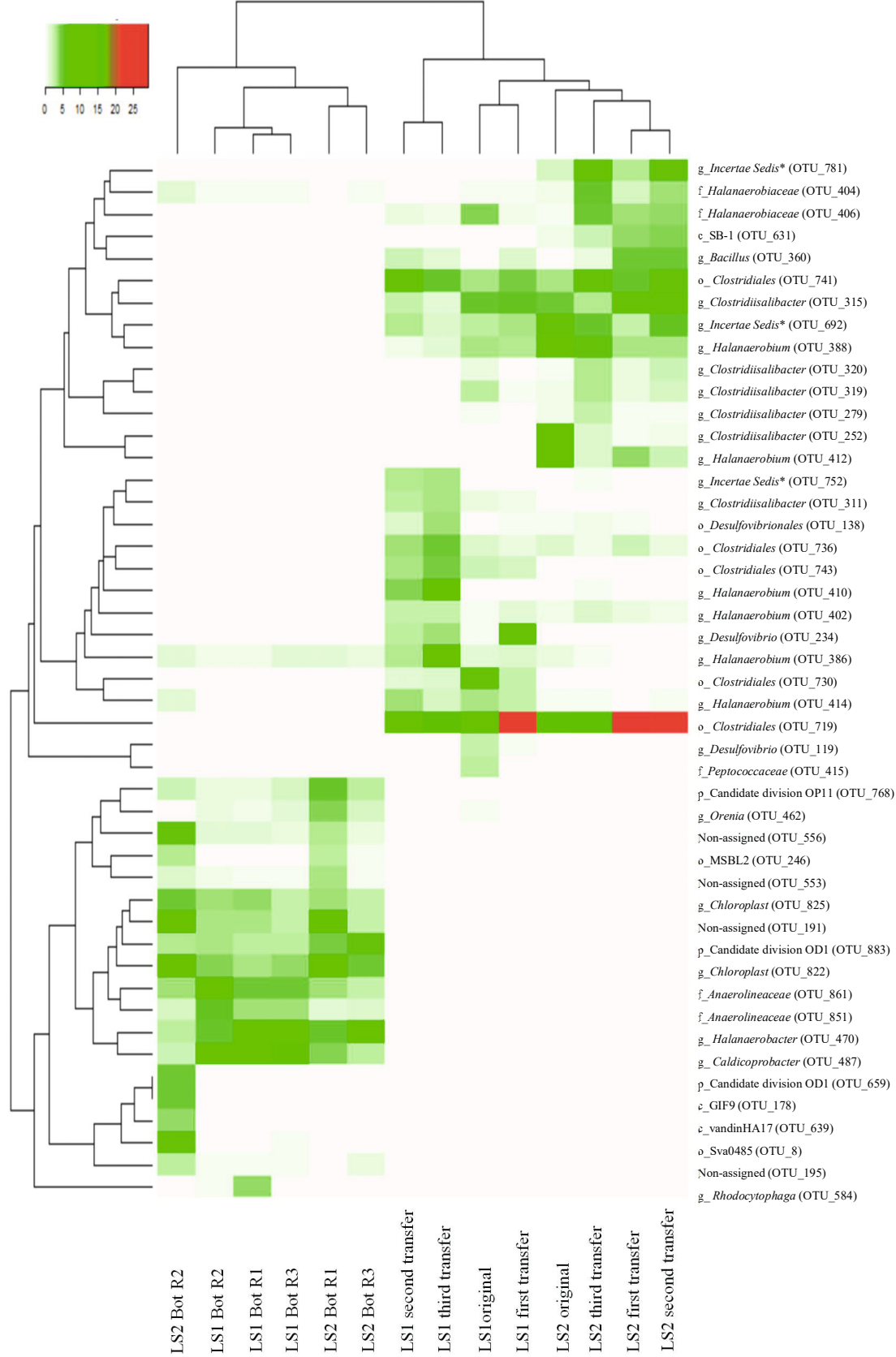

Figure S7.5 Heatmap of OTUs (relative abundance in at least one sample $>2.5 \%$ ) derived from bacterial $16 \mathrm{~S}$ rRNA gene-based community composition (MiSeq) in sediments of Lake Strawbridge (LS) and during transfers of chloroform-dechlorinating enrichments (enrich) derived from bottom layer (Bot, $>12 \mathrm{~cm}$ depth) sediments in LS. Duplicates sediment cores are labelled as LS1 and LS2. R1-3 are three independent DNA extractions. OTUs 
are defined at a threshold of $99 \%$ (maximum one nucleotide mismatch is allowed). OTUs that could be assigned to class, order, family and genus level are indicated with c_, o_, f_ and $g_{-}$prefixes, respectively. *OTU_781, OTU_692 and OTU_752 belong to the families of Ruminococcaceae, Peptostreptococcaceae and Erysipelotrichaceae, respectively.

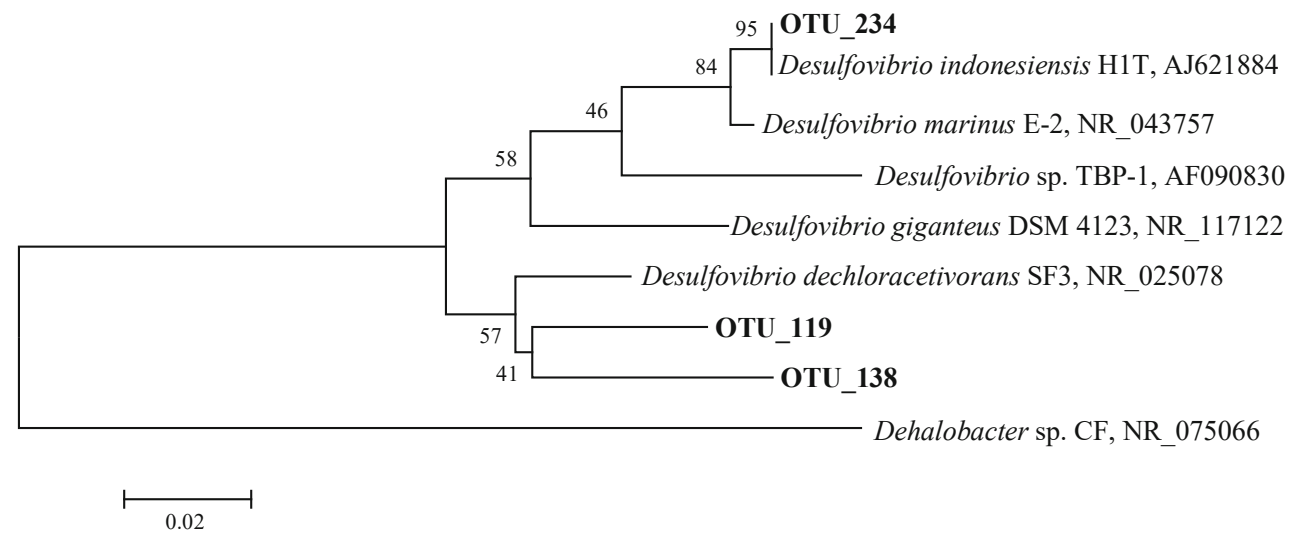

Figure S7.6 Phylogenetic tree of Desulfovibrionales 16S rRNA gene sequences derived from MiSeq dataset. Phylogenetic tree is constructed using the neighbour-joining method in the MEGA 4 program. Reference sequences are downloaded from NCBI and trimmed for sequence alignment with the concatenated sequences (200 bp) from merging pair-end reads (MiSeq). OTUs found in this study are shown in bold. The scale bar represents $2 \%$ sequence divergence. The 16S rRNA gene sequence of the chloroform-degrading Dehalobacter sp. $\mathrm{CF}$ is used as the root. 

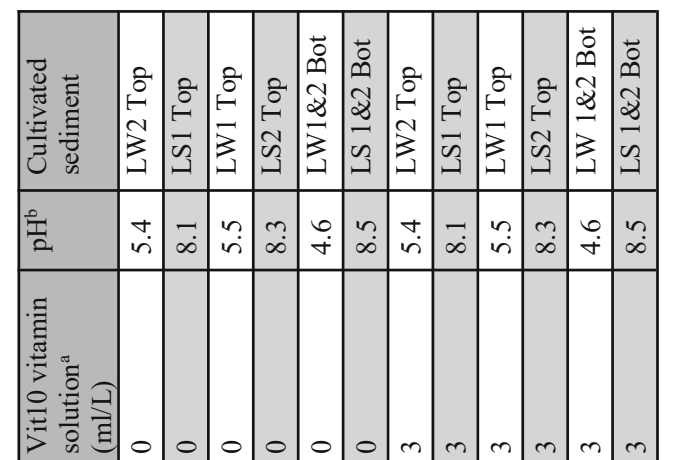

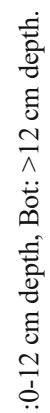

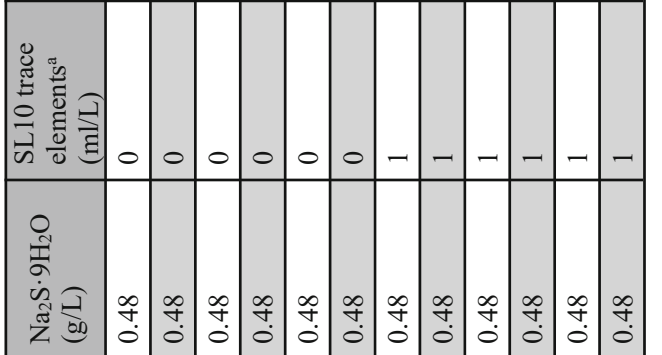

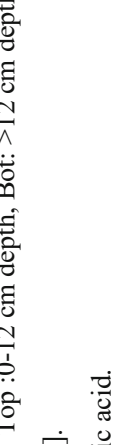

ن
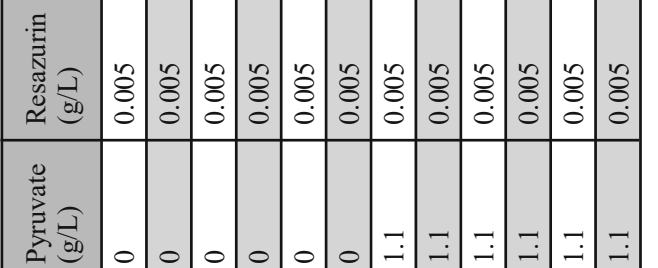

空

焉

के

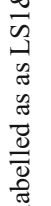

常

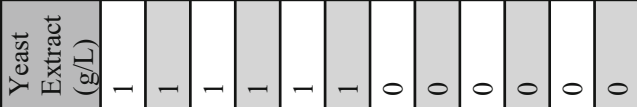

:

ํํำ

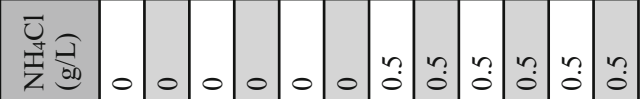

窎

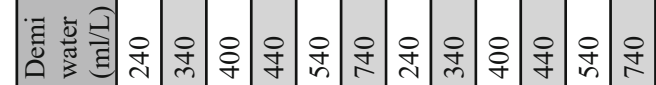

.

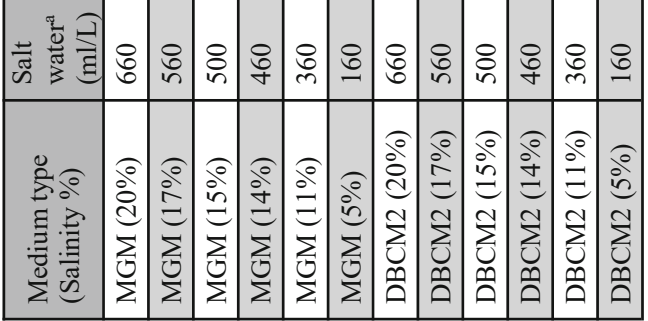

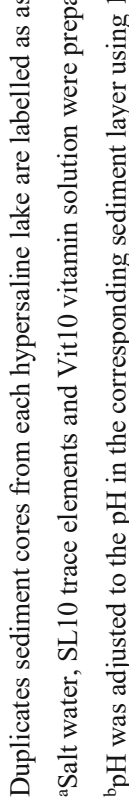




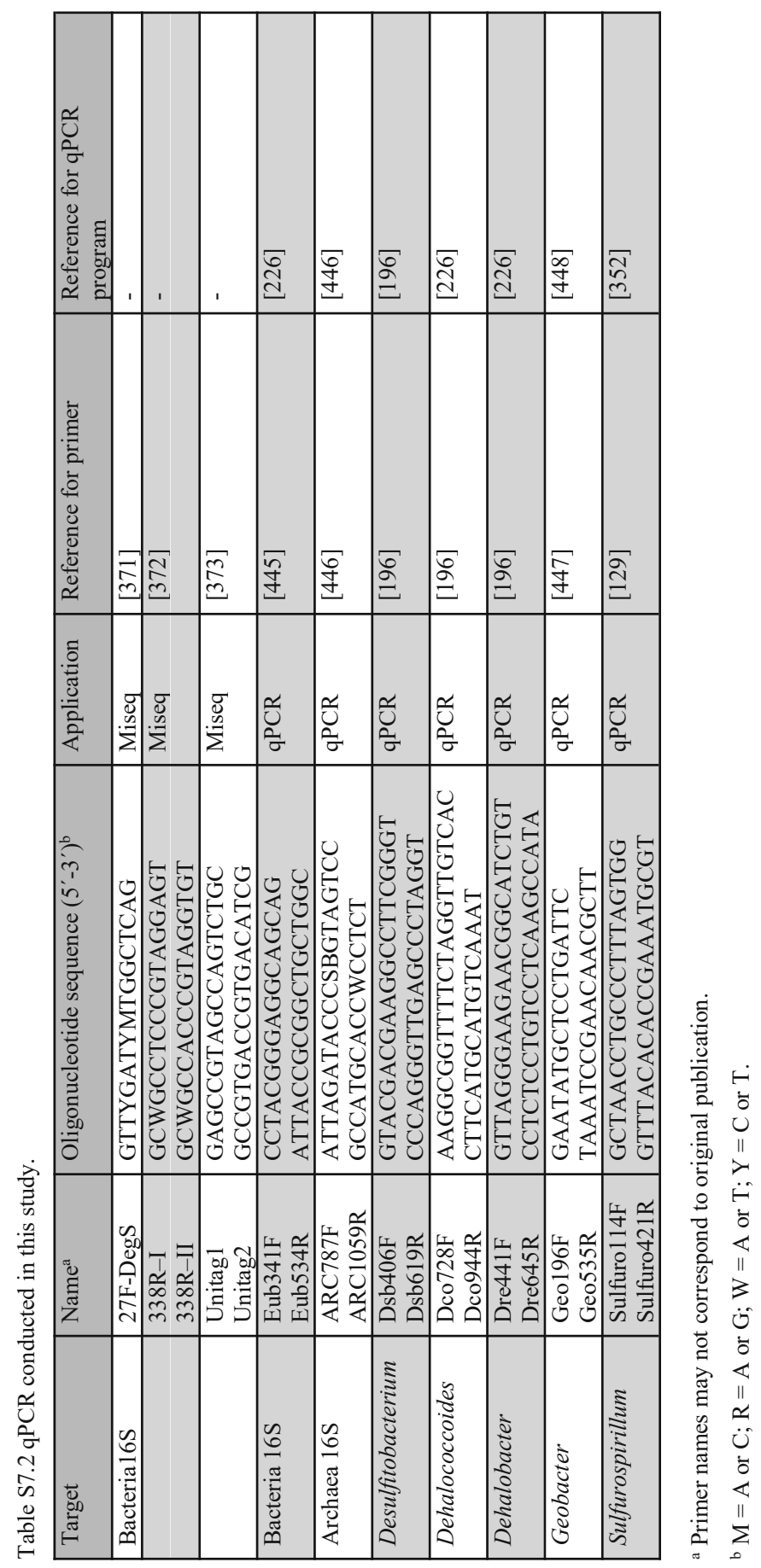




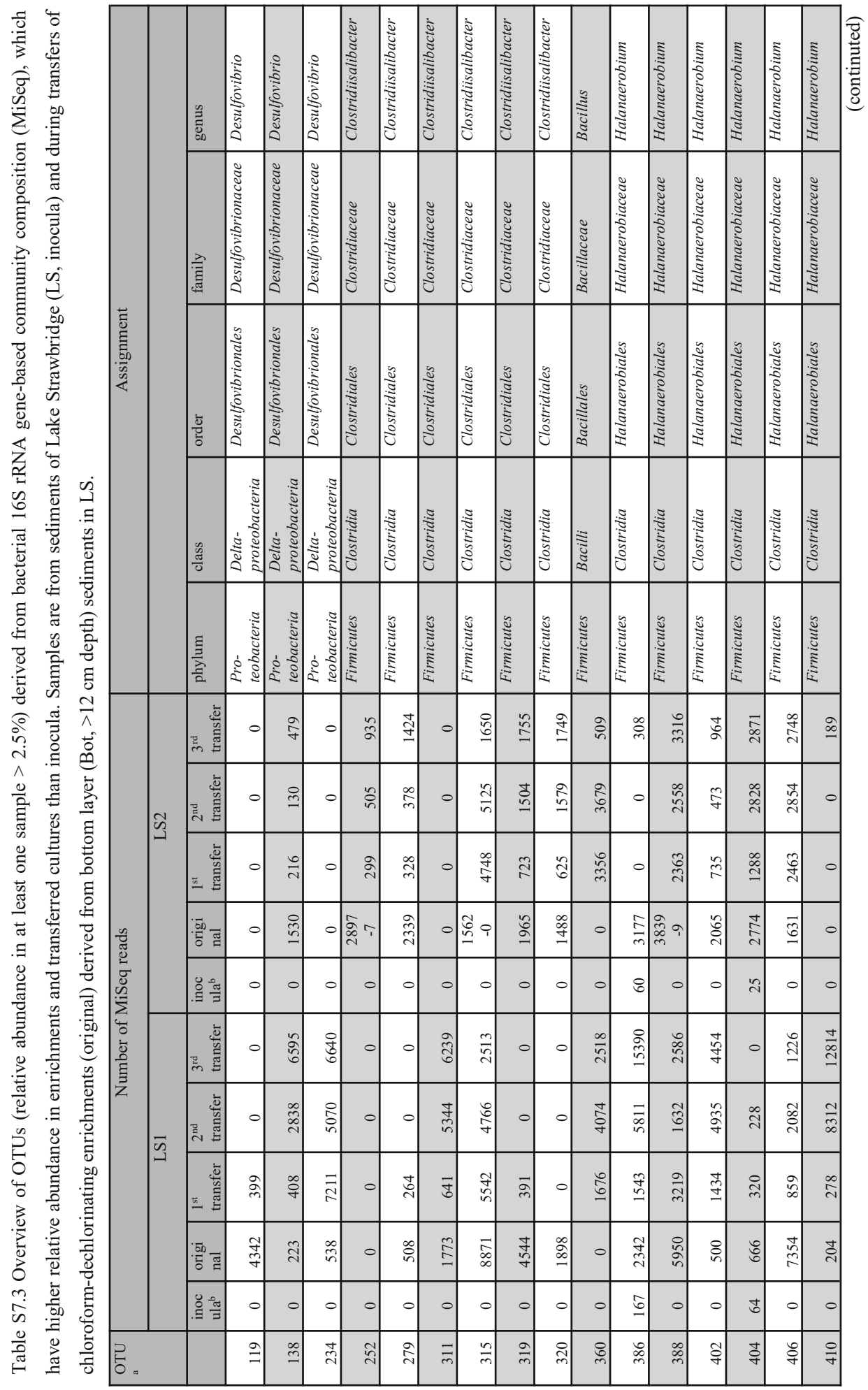




\begin{tabular}{|c|c|c|c|c|c|c|c|c|c|c|c|c|}
\hline 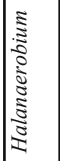 & 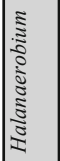 & , & I. & 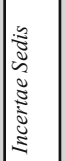 & & 1 & & & 1. & 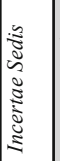 & 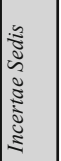 & \\
\hline 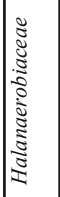 & 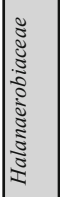 & 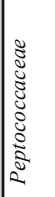 & ' & 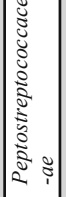 & & 1 & & ( & ' & 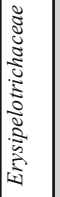 & 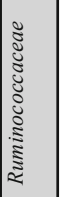 & \\
\hline 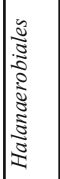 & 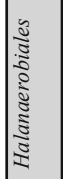 & 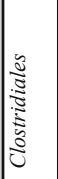 & . & 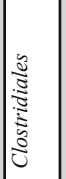 & 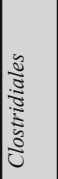 & 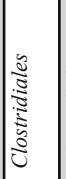 & 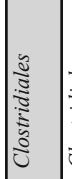 & $\mid$ & $\mid$ & 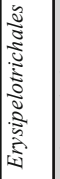 & 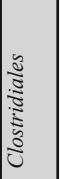 & \\
\hline 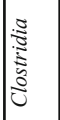 & 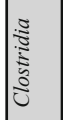 & 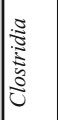 & $\overrightarrow{\tilde{m}}$ & 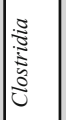 & $\frac{\Im}{\tilde{y}}$ & 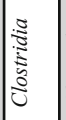 & 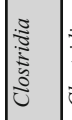 & $\mid$ & 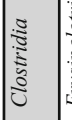 & 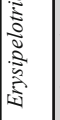 & 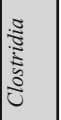 & \\
\hline 苋 & 苞 & 产 & 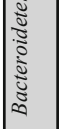 & 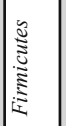 & $\mid$ & $\mid$ & . & $\mid$ & $\mid$ & 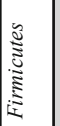 & 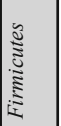 & \\
\hline$\check{\infty}$ & ปे & 0 & $\stackrel{?}{\unlhd}$ & $\begin{array}{l}\stackrel{m}{0} \\
\stackrel{\sim}{\sim}\end{array}$ & ๙ิ่ & 0 & ‡े & ફे & 0 & $\stackrel{\sim}{\sim}$ & 룡 & \begin{tabular}{l}
+ \\
\multirow{2}{*}{} \\
$n$
\end{tabular} \\
\hline$\stackrel{\infty}{0}$ & $\overline{\mathrm{N}}$ & 0 & 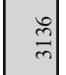 & \begin{tabular}{l}
$:$ \\
$:$ \\
\hdashline
\end{tabular} & $\underset{\Xi}{ \pm}$ & 0 & ๑ొ & $\begin{array}{l}\stackrel{q}{f} \\
f\end{array}$ & 0 & $\widehat{\sigma}$ & $\begin{array}{l}\text { f } \\
f \\
f\end{array}$ & $\begin{array}{l}\text { to } \\
\stackrel{\alpha}{\sim}\end{array}$ \\
\hline 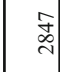 & 0 & 0 & సี & $\stackrel{?}{=}$ & $\begin{array}{l}\text { ठे } \\
\text { ते }\end{array}$ & 0 & : & 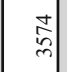 & 0 & 0 & $\frac{\mathrm{O}}{\mathrm{N}}$ & $\begin{array}{l}\text { } \\
\stackrel{+}{2}\end{array}$ \\
\hline 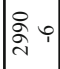 & $\stackrel{8}{=}$ & $\infty$ & ఫे & $\frac{n}{2} m$ & $\begin{array}{ll}\vec{b} \\
i n\end{array}$ & 0 & $\frac{m}{n}$ & 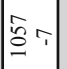 & 0 & 0 & $\left.\begin{array}{l}0 \\
0 \\
0 \\
n\end{array}\right]$ & 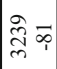 \\
\hline 0 & 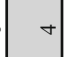 & 0 & 0 & 0 & 0 & 0 & 0 & $\circ$ & 0 & 0 & 은 & $\underset{i}{t}$ \\
\hline 0 & $\stackrel{\circ}{\stackrel{m}{m}}$ & 0 & 0 & \begin{tabular}{l}
$n$ \\
\multirow{2}{*}{} \\
\end{tabular} & 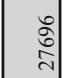 & 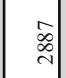 & 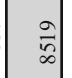 & สิ & $\begin{array}{l}\mathfrak{w} \\
\mathfrak{f} \\
\infty\end{array}$ & जे & 0 & 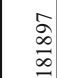 \\
\hline 0 & $\begin{array}{l}\stackrel{0}{2} \\
\stackrel{n}{7}\end{array}$ & 0 & 0 & 릴 & ते & 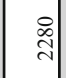 & $\frac{8}{8}$ & స్ำ & $\stackrel{\infty}{\square}$ & हैं & 0 & के \\
\hline 0 & 索 & 0 & 0 & 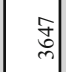 & $\begin{array}{l}\vec{d} \\
\text { స్ }\end{array}$ & $\overrightarrow{\mathrm{n}}$ & $\bar{\sigma}$ & $\begin{array}{l}0 \\
0 \\
0 \\
+\end{array}$ & ڤ્ণે & $\stackrel{+}{=}$ & 0 & $\begin{array}{l}0 \\
\stackrel{2}{0} \\
0\end{array}$ \\
\hline 0 & 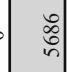 & Ő & 0 & 索 & $\begin{array}{ll}\infty & \\
\tilde{d}^{2} & ?\end{array}$ & $\stackrel{0}{\varrho}$ & సે & $\begin{array}{l}\infty \\
\vec{n}\end{array}$ & $\hat{\tilde{m}}$ & 0 & 0 & $\stackrel{ }{\stackrel{2}{ }}=$ \\
\hline 0 & a & 0 & 0 & 0 & 0 & 0 & 0 & 0 & 0 & 0 & శ్ & 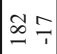 \\
\hline 7 & $\stackrel{\Xi}{\vec{\gamma}}$ & $\stackrel{n}{\sigma}$ & $\overrightarrow{6}$ & ถี & $\frac{2}{2}$ & 익 & $\approx$ & $\vec{\ddagger}$ & $\stackrel{\text { f }}{\rightarrow}$ & กิ & $\stackrel{\overrightarrow{0}}{\sim}$ & $\overline{\Xi 5}$ \\
\hline
\end{tabular}

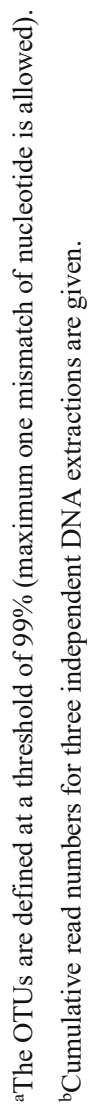


General discussion 


\section{Isolation of organohalide-respiring bacteria (OHRB) and their environmental distribution}

OHRB are a special group of bacteria which are able to couple anaerobic respiration of halogenated organic compounds to energy conservation in a process termed as organohalide respiration (OHR) [7, 10]. Traditional culture-based microbiological methods, e.g. selective cultivation and strain isolation, have provided fundamental insights into physiology, biochemistry and genetics of this intriguing bacterial group. Up to date, 75 organohaliderespiring isolates belonging to 14 different genera in three bacterial phyla, i.e. Chloroflexi, Firmicutes and Proteobacteria, have been reported, of which 73 have been summarized in Chapter 2. Two new organohalide-respiring isolates were published after July 2015. One of these is Dehalococcoides sp. UCH007 derived from contaminated groundwater in Japan [449]. This bacterium dechlorinates trichloroethene, cis-1,2-dichloroethene (DCE) and vinyl chloride to non-toxic ethene [449]. Another isolate is Dehalobacter sp. UNSWDHB that was obtained from a contaminated aquifer near Sydney, Australia, and was shown to dechlorinate chloroform and chlorinated ethanes [450]. This new isolate added another continent from which OHRB have been isolated to date, further supporting their ubiquitous occurrence discussed in Chapter 2. Nevertheless, currently available isolates still represent only a very small fraction of the total diversity of OHRB that exist in the environments. High-throughput culturing approaches that aim at better mimicking in situ conditions, including the iChip [451] and the Microdish culture chip [452], have more recently been exploited to cultivate and/or isolate previously 'uncultivable' microbes. Considering that OHRB are fastidious microbes and mostly strict anaerobes, however, it remains to be demonstrated in future if such innovative cultivation approaches will allow improved cultivation and isolation of OHRB.

Up to date, most of the OHRB have been isolated from organohalide-contaminated environments (Chapter 2), however, an increasing number of reports show that OHRB are widely present in pristine environments, where they are believed to use naturally-occurring organohalides as electron acceptors. For instance, organohalide-respiring Chloroflexi were detected in 116 pristine soil samples collected in the USA [179]. Furthermore, members of the so-called Tidal Flat Clusters within the Dehalococcoidia that are capable of tetrachloroethene (PCE) dechlorination were enriched from marine sediments in Jade Bay, Germany [49]. More recently, bacteria belonging to the class of Dehalococcoidia were detected in deep anoxic waters of a meromictic crater lake, Lake Pavin in France [433]. However, to our knowledge, OHRB in pristine habitats with more extreme conditions such as those characterized by 
acidic/alkaline $\mathrm{pH}$ and high salinity have not been studied before. To fill this knowledge gap, in Chapter 7, we reported reductive dechlorination of chloroform and PCE in anoxic microcosms prepared from sediments collected from the hypersaline Lake Strawbridge, Western Australia. This provided for the first time evidence for the presence of yet unknown OHRB in pristine hypersaline lakes. Considering the previously reported biological organohalide formation in such ecosystems $[39,434,435]$, they provide an intriguing example of habitats potentially hosting interdependent biological chlorination and dechlorination in local halogen cycles and can serve as an interesting, yet untapped source for isolation of novel OHRB.

\section{Detecting uncultured OHRB from environments}

As summarized in Chapter 2, the application of culture-independent methods has highly facilitated the investigation of potential OHRB and their environmental distribution. This has led to the discovery of a broad range of potentially novel ORHB taxa. For instance, uncultured Lahn Clusters [48] and Tidal Flat Clusters [49] were both identified as PCE dechlorinators via combined application of RNA-based stable isotope probing and T-RFLP analysis, and were found only distantly affiliated to cultivated Dehalococcoidia, namely Dehalococcoides mccartyi, Dehalogenimonas spp. and Dehalobium chlorocoercia. Those discoveries have broadened our knowledge of the phylogenetic diversity of OHRB. Nevertheless, these and similar microbial ecological studies such as those described in this thesis (Chapter 5-7) used approaches that rely on the characterization of the microbial $16 \mathrm{~S}$ rRNA (gene) pool. Such approaches have biases due to lack of univerdal coverage of primers that might overlook poorly/un-amplified bacterial groups. Therefore, primer-independent molecular approaches, including various 'omics' methods [13], have been applied for the detection and characterization of potential OHRB in environmental samples. For example, single-cell genomic studies on marine Dehalococcoidia [44, 45] did not reveal OHR associated genes, implying alternative metabolic modes for otherwise metabolically restricted Dehalococcoidia. It cannot be excluded, however, that because complete genomes are hardly retrieved by single-cell genome sequencing, genes might have been missed using this approach $[44,45]$. In contrast, using single-cell sorting and subsequent single cell genome analysis, a recent study identified an $r d h A$ from marine Anaerolineales suggesting that $\mathrm{OHR}$ is not limited to members of the Dehalococcoidia within the Chloroflexi [453]. Metagenomics was also applied to study OHRB, and to exam the metabolic processes and putative interactions within defined mixed cultures and dechlorinating microbial community $[66,237$, 
244, 303]. As with single-cell genomics-based approaches, to achieve complete genomes of OHRB, additional experimental efforts such as primer walking and subsequent amplicon sequencing are required to clarify ambiguities and to achieve gap closure and genome polishing [303]. Therefore, combined application of primer-dependent techniques, single-celland metagenomic surveys is instrumental in gaining a comprehensive understanding of the physiological and genomic characteristics of OHRB and their environmental distribution.

\section{Organohalide substrate spectrum and biotransformation pathways}

Even though diverse organohalides are present in the environment, most studies so far have focussed on biotransformation of organochlorine compounds and to a lesser extent on organobromine, organofluorine and organoiodine compounds. Also in our studies (Chapter 4-7), only OHR of chlorinated aliphatic (chlorinated ethenes, chloroform) and aromatic (chlorinated benzenes, CBs) compounds was investigated. Based on genome information different OHRB, a broad substrate spectrum with respect to OHR has been suggested [23]. However, only limited types of organohalides can be commercially purchased for research. Furthermore, most OHRB are strict anaerobes and grow with low biomass yields, which makes it difficult to test all available organohalides via traditional culture-based microbiological methods, and hence known OHRB have in most cases been only tested for a limited spectrum of potential substrates. In addition, the lack of correlation between sequence similarity of reductive dehalogenases (RDase) encoding genes and substrate specificity, i.e. the lack of robust structure-function relationships, has hindered the ability to confidently predict possible substrate spectra for novel RDase genes as discussed in Chapter 3 . Interestingly several recent studies provided new leads towards possible structure-function relationships that might be useful for the prediction of organohalide substrate spectra for OHRB and their preferred biotransformation pathways. Elucidation of the crystal structures of two RDases for the first time indicated that substrate size might be an important factor, as a 'letterbox' with limited space and deeply buried corrinoid cofacter restrict the range of substrates able to enter and bind to the RDase active site [152, 454]. Furthermore, phylogenetic clustering of novel RDase amino acid sequences with those of characterized RDases was suggested as a useful tool to predict their functional specificity [12]. Another factor that can affect the substrate range and associated pathways is toxicity caused by the hydrophobic nature of substrates as revealed in the study of dehalogenation of bromophenol blue and tetrabromobisphenol A by Dehalococcoides mccartyi CBDB1 [455], and thermodynamic properties of substrates [456]. In line with the latter, thermodynamically more 
favorable reactions were found to be selectively followed by CBs-dechlorinating microcosms as described in Chapter 5.

\section{Corrinoid auxotrophy}

Corrinoid is an essential cofactor for RDase, the key enzymes mediating microbial reductive dehalogenation. Corrinoid co-factors were found in all studied reductive dehalogenases except for the chlorobenzoate RDase functionally characterized from Desulfomonile tiedjei that harbors a heme cofactor [255]. Some facultative OHRB such as Sulfurospirillum multivorans [297], Geobacter lovleyi [128] and Desulfitobacterium hafniense [74, 301] are capable of de novo corrinoid biosynthesis. In contrast, Dehalobacter restrictus as an obligate OHRB is known as a corrinoid auxotroph. In Chapter 4, we confirmed the corrinoid biosynthetic pathway in D. restrictus PER-K23 ${ }^{\mathrm{T}}$ that is non-functional due to a 101-bp truncation in $c b i H$ gene $[67,261]$. Cobalamin is routinely supplied in the growth media during the isolation and maintenance of $D$. restrictus PER-K23 ${ }^{\mathrm{T}}$ in the laboratory, and it is tempting to speculate that the frameshift mutation in $c b i H$ gene arose during long-term cultivation under cobalamin-rich conditions, and decay of the corrinoid biosynthesis pathway as an unused but costly trait. As this mutation is not found in other Dehalobacter spp. genomes, it would be interesting to study their response to corrinoid starvation. This has, however, not been investigated to date since sufficient corrinoid is always supplied during cultivation [55, 63, 269, 450].

Among the important elements in controlling corrinoid uptake and metabolism, five cobalamin riboswitches (Cbl-RS) were identified in D. restrictus PER-K23 ${ }^{\mathrm{T}}$, which regulated the corrinoid metabolism at the transcription level (Chapter 4). The specificity of Cbi-RS to a particular corrinoid derivative was observed before in Desulfitobacterium hafniense [301]. It would be interesting to further investigate the substrate specificity of Cbi-RS found in $D$. restrictus PER-K23 ${ }^{\mathrm{T}}$, which might help to reveal their interaction with particular corrinoid derivatives biosynthesized by co-existing microbes. The importance of gaining additional information on the in situ functionality of Dehalobacter-like populations is further reinforced by their involvement in the dechlorination of CBs in Chapter 5, indicating their potential to be applied in future bioremediation of anoxic contaminated environments.

Remodelling the lower ligand of phenolic corrinoids with 5,6-dimethylbenzimidazole (DMB) to produce the functional corrinoid cofactor, cobalamin, was reported before in Dehalococcoides mccartyi, which is also known as corrinoid auxotroph because of the lack of a complete de novo corrinoid biosynthetic pathway [93-95, 299]. It would be interesting to 
know if remodelling is a shared trait between restricted OHRB i.e. if Dehalobacter species also apply such a corrinoid remodelling strategy, however, this has not been investigated.

Our research in Chapter 4 suggested the importance of the application of functional analyses (e.g. transcriptional analysis and proteomics) in corrinoid metabolism studies of OHRB, as studies at the DNA level such as 16S rRNA genes amplicon sequencing and (meta)genomics can only answer the question 'who is there?' and 'what might they be able to do', but not 'what are they doing and how?'. To further expand our knowledge of corrinoid metabolism of OHRB in a complex community, 'meta' approaches such as metatranscriptomics [457, 458] and metaproteomics $[459,460]$ can be considered to gain comprehensive understanding of the functional role of OHRB, the active metabolic interaction between OHRB and co-existing microbes, and the strategies OHRB employ to meet their nutritional need such as corrinoids, especially for corrinoid auxotrophic OHRB. However, these approaches are often technically challenging with respect to extraction of high quality RNA and proteins from environmental samples and complicated bioinformatic analysis that demand further wet-lab optimization and computational analysis simplification.

\section{Syntrophic interactions in OHR consortia and the role of non-dehalogenating community members}

Many OHRB can grow in axenic cultures with exogenous supplement of required micro- and macronutrients, whereas in natural habitats they rely on other microbes in the community to fulfil their nutritional needs (see Figure 8.1). Application of synthetic/enriched co-cultures as simplified model consortia has revealed some syntrophic interactions between OHRB and their companion microbes $[63,276,324,325,461,462]$. The non-dechlorinating partners can stimulate the growth and dehalogenation activities of OHRB by i) providing carbon source (e.g. acetate) and electron donor (e.g. hydrogen) produced by fermenters like Acetobacterium [324] and Desulfovibrio [242, 324, 325]; ii) providing essential growth factors like corrinoids by methanogens and acetogens [299], Desulfovibrio [324, 325], Geobacter [93], and Sedimentibacter [63]. Other goods and services include vitamin K by provided by an Enterococcus [166], iii) and removal of carbon monoxide (CO) by CO-oxidizing bacteria such as Desulfovibrio vulgaris Hildenborough that D. mccartyi produces but cannot tolerate [37]. In return, OHRB may stimulate growth of non-dechlorinators by detoxifying notorious organohalides [63]. Furthermore, it is possible that OHRB provide additional goods/services in return to non-dechlorinators that have not been discovered yet. In addition to those 


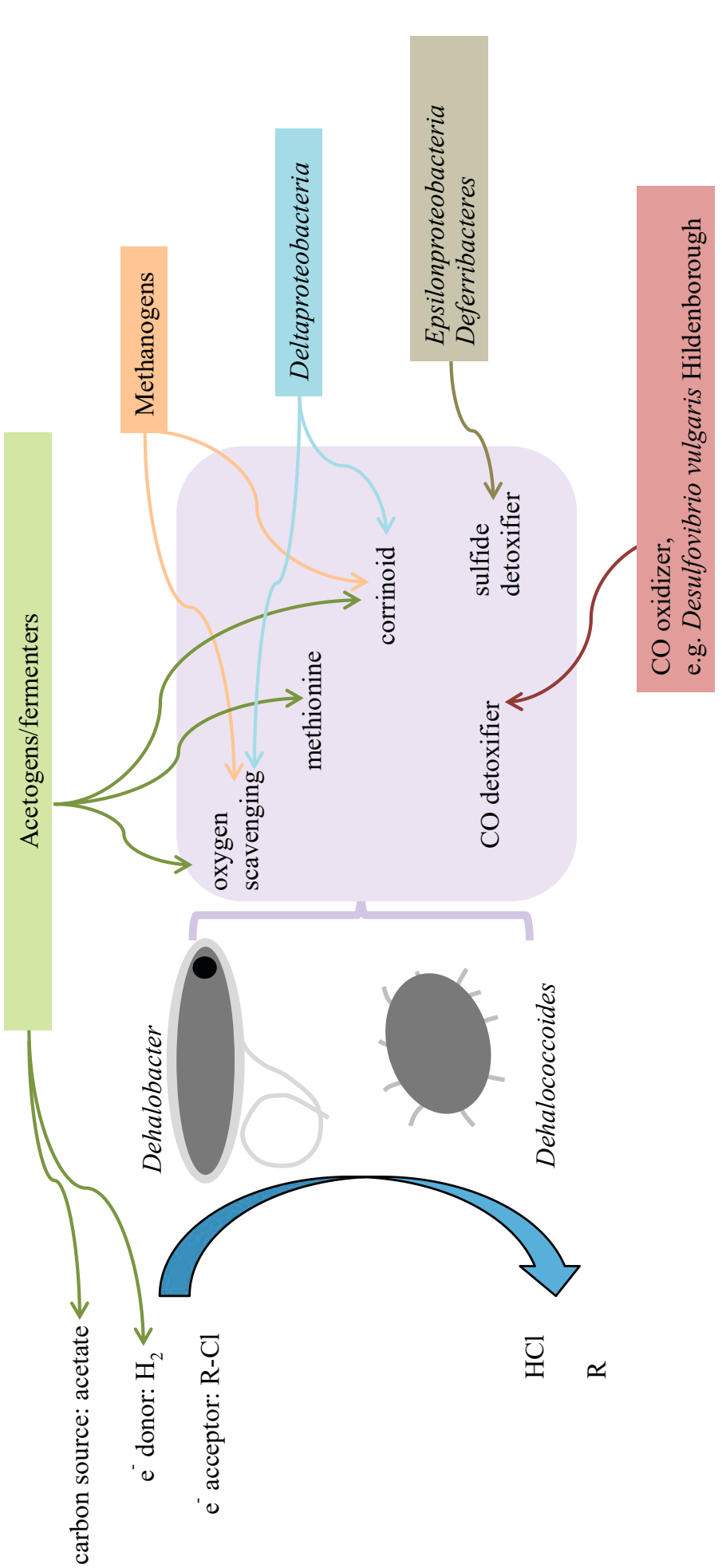


syntrophic interactions between OHRB and non-dechlorinators mentioned above, more potential interactions were discovered based on (meta)genomics studies of the metabolism of OHRB. For instance, the comparative metagenomics of three Dehalococcoides-containing enrichment consortia suggested Deltaproteobacteria and/or acetogens/fermenters as oxygen scavengers and methionine providers for Dehalococcoides [237].

Perhaps one of the most frequently encountered guilds in organohalide-respiring consortia at lab and field scales comprise the microbes driving the sulfur-cycle (Chapter 5 and $\mathbf{6}$ of this thesis [227, 352]. Sulfide as a product of sulfate reducers is a known inhibitor of OHRB in the laboratory [41, 97, 161] and in situ [415]. Similarly, the abundance of Dehalococcoidia and other organohalide-respiring Chloroflexi was reported to be negatively impacted by reduced sulfur species in lake sediments [463]. In our study described in Chapter 6, we showed a yet unrecognised potential role for putative sulfur-oxidizing bacteria such as Deferribacteres, Epsilonproteobacteria (Sulfuricurvum and Sulfurospirillum) and candidate phylum OD1 under field conditions. These guilds seem to support $D$. mccartyi growth by oxidising sulfide and reducing its toxicity. However, these putative sulfur oxidisers seem to be competitive successors of the more established companions of OHRB, i.e. sulfate reducers and fermenters, and hence might impact OHR negatively. The net impact is not known and its verification remains challenging. Finally, sulfite and thiosulfate were reported before to inhibit OHRB strains like Desulfomonile tiedjei $\mathrm{DCB}-1^{\mathrm{T}}[11,136]$, which suggest microbes in the community that are capable of removing these compounds may stimulate OHR activity.

Members of the Bacteroidetes are reported in many environments such as freshwater, soil, the ocean, and the mammalian gut, all of which are characterized by the presence of a large spectrum of complex organic matter (e.g. proteins and carbohydrates) [464]. We found an exceptionally high relative abundance of members of the Bacteroidetes in CBs-dechlorinating microbial communities (with average relative abundance of $79.7 \%$, Chapter 5 ). To test the potential link between predominant Bacteroidetes and $\mathrm{CBs}$ dechlorinators, functional metagenomics studies encompassing metatranscriptomics and metaproteomics can be applied.

\section{Concluding remarks and perspectives}

Bioremediation is considered a sustainable, relatively efficient and cost-effective technology to treat contaminated sites [465]. OHRB continue to draw great scientific interest due to their potential application in bioremediation of organohalide contaminated subsurface environments $[12,13]$. Their presence and activity is pivotal for effective bioremediation, but 
lack of comprehensive information about their metabolism, nutritional requirements and interactions with geochemical geophysical parameters limits in situ implementation. The knowledge generated in this thesis that can be used to guide design and optimization of bioremediation strategies is summarized in Figure 8.2.

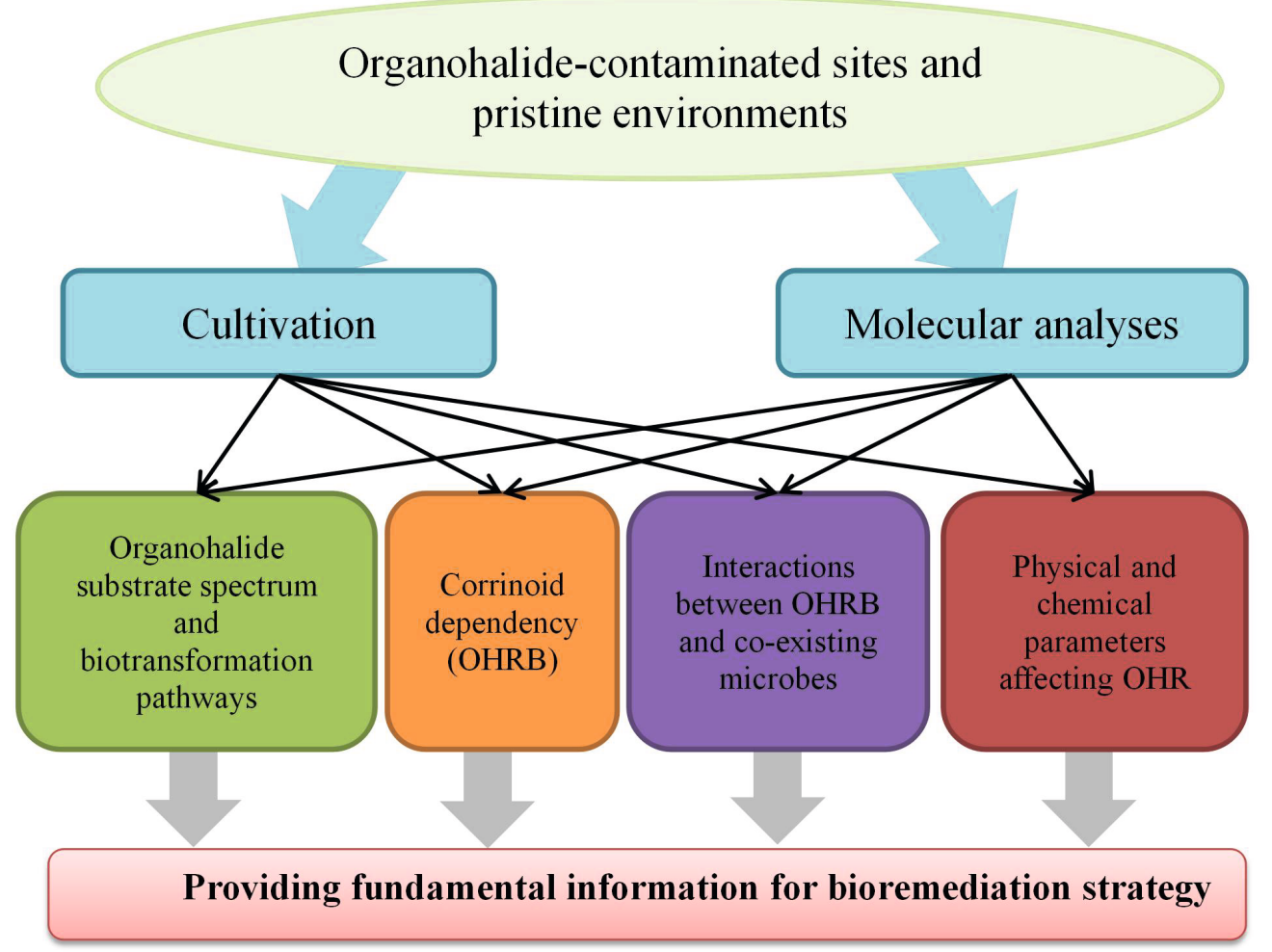

Figure 8.2 Overview of this study contributes to the bioremediation strategy.

As reviewed in Chapter 2, many environmental factors can affect the distribution and activity of OHRB, such as redox conditions, micronutrients, electron acceptors and interaction with chemical species, which further impact the outcome of bioremediation. An intriguing factor is DMB as essential lower ligand of a functional corrinoid cofactor (cobalamin) for RDases in the obligate OHRB D. mccartyi and Dehalobacter. It is likely that this compound will be used in attempts to biostimulate in situ reductive dehalogenation. However, DMB is known to enforce some community members to produce cobalamin that is non-functional for them. A recent example is the case of S. multivorans [145] which requires norpseudo-B12 as corrinoid cofactor [144]. Hence stimulating D. mccartyi guilds can on the other hand prohibit $S$. 
multivorans which as discussed in Chapter $\mathbf{2}$ can play multiple beneficiary roles in bioremediation of chlorinated ethene.

The enrichment cultures we obtained in Chapter 7 seem to be well adapted to alkaline $\mathrm{pH}$ and high salinity in hypersaline sediments. Besides serving as a source for isolating novel OHRB, the enriched consortia could be a good option for bioremediation of contaminated sites with harsh $\mathrm{pH}$ and/or salinity, such as hypersaline wastewater [466] and/or alkaline wastewater if they are contaminated with target organohalides compounds.

Studies described in this thesis contribute to expanding our understanding of the ecophysiology and environmental distribution of OHRB from different angles, such as the presence of $\mathrm{OHRB}$ in pristine extreme environments, the corrinoid auxotrophy of OHRB, and their syntrophic interactions with co-existing, non-dechlorinating microbes. Our research provides knowledge for evaluating and optimizing bioremediation strategies and contributes to building a scientific foundation for regulatory bodies and companies to improve the efficacy of the bioremediation of organohalide pollutants. 


\section{References}

1. Gribble, G.W., The diversity of naturally produced organohalogens. Chemosphere, 2003. 52(2): p. 289297.

2. Gribble, G.W., Naturally occurring organohalogen compounds-a comprehensive update. 2010: Springer

3. Stringer, R. and P. Johnston, Chlorine and the environment: An overview of the chlorine industry. 2001: Springer.

4. Ritter, L., et al., A review of selected persistent organic pollutants. Available from: http://cdrwww.who.int/ipcs/assessment/en/pcs $95 \quad 39$ 2004 05 13.pdf. 1995. 65: p. 66.

5. Häggblom, M.M. and I.D. Bossert, Halogenated organic compounds-a global perspective, in Dehalogenation. 2004, Springer. p. 3-29.

6. $\quad$ Edwards, E.A., Breathing the unbreathable. Science, 2014. 346(6208): p. 424-425.

7. Leys, D., L. Adrian, and H. Smidt, Organohalide respiration: microbes breathing chlorinated molecules. Phil Trans R Soc B, 2013. 368(1616): p. 20120316.

8. Field, J.A. and R. Sierra-Alvarez, Microbial degradation of chlorinated benzenes. Biodegradation, 2008. 19(4): p. 463-480.

9. Zinder, S., Anaerobic utilization of halohydrocarbons, in Handbook of Hydrocarbon and Lipid Microbiology. 2010, Springer. p. 2049-2064.

10. Smidt, H. and W.M. de Vos, Anaerobic microbial dehalogenation. Annu Rev Microbiol, 2004. 58: p. 43-73.

11. DeWeerd, K.A., et al., Desulfomonile tiedjei gen. nov. and sp. nov., a novel anaerobic, dehalogenating, sulfate-reducing bacterium. Arch Microbiol, 1990. 154(1): p. 23-30.

12. Jugder, B.-E., et al., Organohalide respiring bacteria and reductive dehalogenases: key tools in organohalide bioremediation. Front Microbiol, 2016. 7: p. 249.

13. Maphosa, F., W.M. de Vos, and H. Smidt, Exploiting the ecogenomics toolbox for environmental diagnostics of organohalide-respiring bacteria. Trends Biotechnol, 2010. 28(6): p. 308-16.

14. Stroo, H.F., et al., Bioaugmentation with Dehalococcoides: A decision guide, in Bioaugmentation for Groundwater Remediation. 2013, Springer. p. 117-140.

15. Marzorati, M., et al., Identification of molecular markers to follow up the bioremediation of sites contaminated with chlorinated compounds. Methods Mol Biol, 2010. 668: p. 219-234.

16. Caporaso, J.G., et al., Ultra-high-throughput microbial community analysis on the Illumina HiSeq and MiSeq platforms. ISME J, 2012. 6(8): p. 1621-1624.

17. Goodwin, S., J.D. McPherson, and W.R. McCombie, Coming of age: ten years of next-generation sequencing technologies. Nat Rev Genet, 2016. 17: p. 331-351.

18. Liu, L., et al., Comparison of next-generation sequencing systems. BioMed Res Int, 2012. 2012: p. 251364.

19. Deakin, C.T., et al., Impact of next-generation sequencing error on analysis of barcoded plasmid libraries of known complexity and sequence. Nucleic Acids Res, 2014. 42(16): p. e129.

20. Kim, S.-H., et al., Genome sequence of Desulfitobacterium hafniense DCB-2, a Gram-positive anaerobe capable of dehalogenation and metal reduction. BMC Microbiol, 2012. 12(1): p. 21.

21. Lee, P.K., et al., Comparative genomics of two newly isolated Dehalococcoides strains and an enrichment using a genus microarray. ISME J, 2011. 5(6): p. 1014-1024.

22. West, K.A., et al., Comparative genomics of "Dehalococcoides ethenogenes" 195 and an enrichment culture containing unsequenced "Dehalococcoides" strains. Appl Environ Microbiol, 2008. 74(11): p. 3533-3540.

23. Richardson, R.E., Genomic insights into organohalide respiration. Curr Opin Biotechnol, 2013. 24(3): p. 498-505.

24. Schiffmann, C.L., et al., Proteome profile and proteogenomics of the organohalide-respiring bacterium Dehalococcoides mccartyi strain CBDB1 grown on hexachlorobenzene as electron acceptor. J Proteomics, 2014. 98: p. 59-64.

25. Goris, T., et al., Proteomics of the organohalide-respiring Epsilonproteobacterium Sulfurospirillum multivorans adapted to tetrachloroethene and other energy substrates. Sci Rep, 2015. 5.

26. Kruse, T., et al., Genomic, proteomic, and biochemical analysis of the organohalide respiratory pathway in Desulfitobacterium dehalogenans. J Bacteriol, 2015. 197(5): p. 893-904.

27. Pöritz, M., et al., Dehalococcoides mccartyi strain DCMB5 respires a broad spectrum of chlorinated aromatic compounds. Appl Environ Microbiol, 2015. 81(2): p. 587-596.

28. Morris, R., et al., Comparative proteomics of Dehalococcoides spp. reveals strain-specific peptides associated with activity. Appl Environ Microbiol, 2007. 73(1): p. 320-326. 
29. Prat, L., et al., Physiological adaptation of Desulfitobacterium hafniense strain TCE1 to tetrachloroethene respiration. Appl Environ Microbiol, 2011. 77(11): p. 3853-3859.

30. Nijenhuis, I., et al., Stable isotope fractionation of tetrachloroethene during reductive dechlorination by Sulfurospirillum multivorans and Desulfitobacterium sp. strain PCE-S and abiotic reactions with cyanocobalamin. Appl Environ Microbiol, 2005. 71(7): p. 3413-3419.

31. Cichocka, D., et al., Factors controlling the carbon isotope fractionation of tetra-and trichloroethene during reductive dechlorination by Sulfurospirillum ssp. and Desulfitobacterium sp. strain PCE-S. FEMS Microbiol Ecol, 2007. 62(1): p. 98-107.

32. Cretnik, S., et al., Reductive dechlorination of TCE by chemical model systems in comparison to dehalogenating bacteria: insights from dual element isotope analysis $\left({ }^{13} \mathrm{C} /{ }^{12} \mathrm{C},{ }^{37} \mathrm{Cl} /{ }^{35} \mathrm{Cl}\right)$. Environ Sci Technol, 2013. 47: p. 6855-6863.

33. Wiegert, C., et al., Carbon and chlorine isotope fractionation during microbial degradation of tetraand trichloroethene. Environ Sci Technol, 2013. 47(12): p. 6449-6456.

34. Badin, A., et al., Multiple dual $\mathrm{C}-\mathrm{Cl}$ isotope patterns associated with reductive dechlorination of tetrachloroethene. Environ Sci Technol, 2014. 48(16): p. 9179-9186.

35. Renpenning, J., I. Rapp, and I. Nijenhuis, Substrate hydrophobicity and cell composition influence the extent of rate limitation and masking of isotope fractionation during microbial reductive dehalogenation of chlorinated ethenes. Environ Sci Technol, 2015. 49(7): p. 4293-4301.

36. Adrian, L. and E. Marco-Urrea, Isotopes in geobiochemistry: tracing metabolic pathways in microorganisms of environmental relevance with stable isotopes. Curr Opin Biotechnol, 2016. 41: p. 19-25.

37. Zhuang, W.Q., et al., Incomplete Wood-Ljungdahl pathway facilitates one-carbon metabolism in organohalide-respiring Dehalococcoides mccartyi. Proc Natl Acad Sci USA, 2014. 111(17): p. 64196424.

38. Wiegert, C., et al., Dual carbon-chlorine stable isotope investigation of sources and fate of chlorinated ethenes in contaminated groundwater. Environ Sci Technol, 2012. 46(20): p. 10918-10925.

39. Rücker, A., et al., Predominance of biotic over abiotic formation of halogenated hydrocarbons in hypersaline sediments in Western Australia. Environ Sci Technol, 2014. 48(16): p. 9170-9178.

40. Löffler, F.E., et al., Dehalococcoides mccartyi gen. nov., sp. nov., obligately organohalide-respiring anaerobic bacteria relevant to halogen cycling and bioremediation, belong to a novel bacterial class, Dehalococcoidia classis nov., order Dehalococcoidales ord. nov. and family Dehalococcoidaceae fam. nov., within the phylum Chloroflexi. Int J Syst Evol Microbiol, 2013. 63: p. 625-35.

41. May, H.D., et al., Dehalorespiration with polychlorinated biphenyls by an anaerobic ultramicrobacterium. Appl Environ Microbiol, 2008. 74(7): p. 2089-2094.

42. Justicia-Leon, S.D., et al., Dichloromethane fermentation by a Dehalobacter sp. in an enrichment culture derived from pristine river sediment. Appl Environ Microbiol, 2012. 78(4): p. 1288-1291.

43. Lee, M., et al., Complete chloroform dechlorination by organochlorine respiration and fermentation. Environ Microbiol, 2012. 14(4): p. 883-894.

44. Kaster, A.-K., et al., Single cell genomic study of Dehalococcoidetes species from deep-sea sediments of the Peruvian Margin. ISME J, 2014. 8: p. 1831-1842.

45. Wasmund, K., et al., Genome sequencing of a single cell of the widely distributed marine subsurface Dehalococcoidia, phylum Chloroflexi. ISME J, 2014. 8(2): p. 383-397.

46. Hug, L.A., et al., Overview of organohalide-respiring bacteria and a proposal for a classification system for reductive dehalogenases. Phil Trans R Soc B, 2013. 368(1616): p. 20120322.

47. Sung, Y., et al., Characterization of two tetrachloroethene-reducing, acetate-oxidizing anaerobic bacteria and their description as Desulfuromonas michiganensis sp. nov. Appl Environ Microbiol, 2003. 69(5): p. 2964-2974.

48. Kittelmann, S. and M.W. Friedrich, Identification of novel perchloroethene - respiring microorganisms in anoxic river sediment by $R N A$ - based stable isotope probing. Environ Microbiol, 2008. 10(1): p. 31 46.

49. Kittelmann, S. and M.W. Friedrich, Novel uncultured Chloroflexi dechlorinate perchloroethene to trans - dichloroethene in tidal flat sediments. Environ Microbiol, 2008. 10(6): p. 1557-1570.

50. Ritalahti, K.M. and F.E. Löffler, Populations implicated in anaerobic reductive dechlorination of 1,2dichloropropane in highly enriched bacterial communities. Appl Environ Microbiol, 2004. 70(7): p. 4088-4095.

51. Löffler, F.E., et al., Complete reductive dechlorination of 1,2-dichloropropane by anaerobic bacteria. Appl Environ Microbiol, 1997. 63(7): p. 2870-2875.

52. Holliger, C., et al., Dehalobacter restrictus gen. nov. and sp. nov., a strictly anaerobic bacterium that reductively dechlorinates tetra- and trichloroethene in an anaerobic respiration. Arch Microbiol, 1998. 169(4): p. 313-321. 
53. Holliger, C., et al., A highly purified enrichment culture couples the reductive dechlorination of tetrachloroethene to growth. Appl Environ Microbiol, 1993. 59(9): p. 2991-7.

54. Wild, A., R. Hermann, and T. Leisinger, Isolation of an anaerobic bacterium which reductively dechlorinates tetrachloroethene and trichloroethene. Biodegradation, 1996. 7(6): p. 507-511.

55. Yoshida, N., et al., A novel Dehalobacter species is involved in extensive 4,5,6,7-tetrachlorophthalide dechlorination. Appl Environ Microbiol, 2009. 75(8): p. 2400-2405.

56. Sun, B., et al., Microbial dehalorespiration with 1,1,1-trichloroethane. Science, 2002. 298(5595): p. 1023-5.

57. Nelson J., J.J., and Zinder S., Dehalogenation of chlorobenzenes, dichlorotoluene, and tetrachloroethene by three Dehalobacter spp. Environ Sci and Technol, 2014. 48(7): p. 3776-3782.

58. Wang, S., et al., Isolation and characterization of a novel Dehalobacter species strain TCP1 that reductively dechlorinates 2, 4, 6-trichlorophenol. Biodegradation, 2014. 25(2): p. 313-323.

59. Grostern, A. and E.A. Edwards, Growth of Dehalobacter and Dehalococcoides spp. during degradation of chlorinated ethanes. Appl Environ Microbiol, 2006. 72(1): p. 428-36.

60. Li, Z., et al., Anaerobic mineralization of 2, 4, 6-tribromophenol to $\mathrm{CO}_{2}$ by a synthetic microbial community comprising Clostridium, Dehalobacter, and Desulfatiglans. Bioresour Technol, 2015. 176: $\mathrm{p}$. 225-232.

61. Grostern, A., et al., Chloroform respiration to dichloromethane by a Dehalobacter population. Environ Microbiol, 2010. 12(4): p. 1053-60.

62. Nelson, J.L., et al., A role for Dehalobacter spp. in the reductive dehalogenation of dichlorobenzenes and monochlorobenzene. Environ Sci and Technol, 2011. 45(16): p. 6806-6813.

63. van Doesburg, W., et al., Reductive dechlorination of $\beta$-hexachlorocyclohexane $(\beta-H C H)$ by a Dehalobacter species in coculture with a Sedimentibacter sp. FEMS Microbiol Ecol, 2005. 54(1): p. 87-95.

64. Maillard, J., et al., Characterization of the corrinoid iron-sulfur protein tetrachloroethene reductive dehalogenase of Dehalobacter restrictus. Appl Environ Microbiol, 2003. 69(8): p. 4628-4638.

65. Rupakula, A., et al., Functional genomics of corrinoid starvation in the organohalide-respiring bacterium Dehalobacter restrictus strain PER-K23. Front Microbiol, 2014. 5: p. 751.

66. Maphosa, F., et al., Metagenome analysis reveals yet unexplored reductive dechlorinating potential of Dehalobacter sp. E1 growing in co-culture with Sedimentibacter sp. Environ Microbiol Rep, 2012. 4(6): p. 604-616.

67. Rupakula, A., et al., The restricted metabolism of the obligate organohalide respiring bacterium Dehalobacter restrictus: lessons from tiered functional genomics. Phil Trans R Soc B, 2013. 368(1616): p. 20150325.

68. Villemur, R., et al., The Desulfitobacterium genus. FEMS Microbiol Rev, 2006. 30(5): p. $706-733$.

69. Madsen, T. and D. Licht, Isolation and characterization of an anaerobic chlorophenol-transforming bacterium. Appl Environ Microbiol, 1992. 58(9): p. 2874-2878.

70. Utkin, I., C. Woese, and J. Wiegel, Isolation and characterization of Desulfitobacterium dehalogenans gen. nov., sp. nov., an anaerobic bacterium which reductively dechlorinates chlorophenolic compounds. Int J Syst Bacteriol, 1994. 44(4): p. 612-619.

71. Christiansen, N. and B.K. Ahring, Desulfitobacterium hafniense sp. nov., an anaerobic, reductively dechlorinating bacterium. Int J Syst Bacteriol, 1996. 46(2): p. 442-448.

72. Bouchard, B., et al., Isolation and characterization of Desulfitobacterium frappieri sp. nov., an anaerobic bacterium which reductively dechlorinates pentachlorophenol to 3-chlorophenol. Int J Syst Bacteriol, 1996. 46(4): p. 1010-1015.

73. Niggemyer, A., et al., Isolation and characterization of a novel As (V)-reducing bacterium: implications for arsenic mobilization and the genus Desulfitobacterium. Appl Environ Microbiol, 2001. 67(12): p. 5568-5580.

74. Nonaka, H., et al., Complete genome sequence of the dehalorespiring bacterium Desulfitobacterium hafniense Y51 and comparison with Dehalococcoides ethenogenes 195. J Bacteriol, 2006. 188(6): p. 2262-2274.

75. Goris, T., et al., Draft genome sequence and characterization of Desulfitobacterium hafniense PCE-S. Stand Genomic Sci, 2015. 10(1): p. 15.

76. Duret, A., C. Holliger, and J. Maillard, The physiological opportunism of Desulfitobacterium hafniense strain TCE1 towards organohalide respiration with tetrachloroethene. Appl Environ Microbiol, 2012. 78(17): p. 6121-6127.

77. Thibodeau, J., et al., Purification, cloning, and sequencing of a 3, 5-dichlorophenol reductive dehalogenase from Desulfitobacterium frappieri PCP-1. Appl Environ Microbiol, 2004. 70(8): p. $4532-$ 4537 . 
78. van de Pas, B.A., et al., Purification and molecular characterization of ortho-chlorophenol reductive dehalogenase, a key enzyme of halorespiration in Desulfitobacterium dehalogenans. J Biol Chem, 1999. 274(29): p. 20287-20292.

79. Suyama, A., et al., Molecular characterization of the PceA reductive dehalogenase of Desulfitobacterium sp. strain Y51. J Bacteriol, 2002. 184(13): p. 3419-3425.

80. Tsukagoshi, N., et al., Isolation and transcriptional analysis of novel tetrachloroethene reductive dehalogenase gene from Desulfitobacterium sp. strain KBC1. Appl Microbiol Biotechnol, 2006. 69(5): p. 543-553.

81. van de Pas, B.A., et al., Two distinct enzyme systems are responsible for tetrachloroethene and chlorophenol reductive dehalogenation in Desulfitobacterium strain PCE1. Arch Microbiol, 2001. 176(3): p. 165-169.

82. Miller, E., G. Wohlfarth, and G. Diekert, Purification and characterization of the tetrachloroethene reductive dehalogenase of strain PCE-S. Arch Microbiol, 1998. 169(6): p. 497-502.

83. Marzorati, M., et al., A novel reductive dehalogenase, identified in a contaminated groundwater enrichment culture and in Desulfitobacterium dichloroeliminans strain DCA1, is linked to dehalogenation of 1, 2-dichloroethane. Appl Environ Microbiol, 2007. 73(9): p. 2990-2999.

84. Maymo-Gatell, X., et al., Isolation of a bacterium that reductively dechlorinates tetrachloroethene to ethene. Science, 1997. 276(5318): p. 1568-1571.

85. LaRoe, S.L., A.D. Fricker, and D.L. Bedard, Dehalococcoides mccartyi strain JNA in pure culture extensively dechlorinates aroclor 1260 according to polychlorinated biphenyl (PCB) Dechlorination Process N. Environ Sci Technol, 2014. 48(16): p. 9187-9196.

86. Bedard, D.L., et al., Development and characterization of stable sediment-free anaerobic bacterial enrichment cultures that dechlorinate Aroclor 1260. Appl Environ Microbiol, 2006. 72(4): p. 24602470 .

87. Wang, S. and J. He, Phylogenetically distinct bacteria involve extensive dechlorination of Aroclor 1260 in sediment-free cultures. PLoS One, 2013. 8(3): p. e59178.

88. Wang, S., et al., Genomic characterization of three unique Dehalococcoides that respire on persistent polychlorinated biphenyls. Proc Natl Acad Sci USA, 2014. 111(33): p. 12103-12108.

89. Wang, S. and J. He, Dechlorination of commercial PCBs and other multiple halogenated compounds by a sediment-free culture containing Dehalococcoides and Dehalobacter. Environ Sci Technol, 2013. 47(18): p. 10526-10534.

90. Hendrickson, E.R., et al., Molecular analysis of Dehalococcoides 16S ribosomal DNA from chloroethene-contaminated sites throughout North America and Europe. Appl Environ Microbiol, 2002. 68(2): p. 485-495.

91. Kube, M., et al., Genome sequence of the chlorinated compound-respiring bacterium Dehalococcoides species strain CBDB1. Nat Biotechnol, 2005. 23(10): p. 1269-1273.

92. Seshadri, R., et al., Genome sequence of the PCE-dechlorinating bacterium Dehalococcoides ethenogenes. Science, 2005. 307(5706): p. 105-108.

93. Yan, J., et al., Unexpected specificity of interspecies cobamide transfer from Geobacter spp. to organohalide-respiring Dehalococcoides mccartyi strains. Appl Environ Microbiol, 2012. 78: p. 66306.

94. Yi, S., et al., Versatility in corrinoid salvaging and remodeling pathways supports corrinoid-dependent metabolism in Dehalococcoides mccartyi. Appl Environ Microbiol, 2012. 78(21): p. 7745-52.

95. Men, Y., et al., Identification of specific corrinoids reveals corrinoid modification in dechlorinating microbial communities. Environ Microbiol, 2015. 17(12): p. 4873-4884.

96. McMurdie, P.J., et al., Localized plasticity in the streamlined genomes of vinyl chloride respiring Dehalococcoides. PLoS Genetics, 2009. 5(11): p. e1000714.

97. Magnuson, J.K., et al., Reductive dechlorination of tetrachloroethene to ethene by a two-component enzyme pathway. Appl Environ Microbiol, 1998. 64(4): p. 1270-1275.

98. Magnuson, J.K., et al., Trichloroethene reductive dehalogenase from Dehalococcoides ethenogenes: Sequence of tceA and substrate range characterization. Appl Environ Microbiol, 2000. 66(12): p. 5141 5147.

99. Chow, W.L., et al., Identification and transcriptional analysis of trans-DCE-producing reductive dehalogenases in Dehalococcoides species. ISME J, 2010. 4(8): p. 1020-1030.

100. Krajmalnik-Brown, R., et al., Genetic identification of a putative vinyl chloride reductase in Dehalococcoides sp. strain BAV1. Appl Environ Microbiol, 2004. 70(10): p. 6347-6351.

101. Müller, J.A., et al., Molecular identification of the catabolic vinyl chloride reductase from Dehalococcoides sp. strain VS and its environmental distribution. Appl Environ Microbiol, 2004. 70(8): p. $4880-4888$. 
102. Parthasarathy, A., et al., Biochemical and EPR-spectroscopic investigation into heterologously expressed vinyl chloride reductive dehalogenase (VcrA) from Dehalococcoides mccartyi strain VS. J Am Chem Soc, 2015. 137(10): p. 3525-3532.

103. Adrian, L., et al., Identification of a chlorobenzene reductive dehalogenase in Dehalococcoides $s p$. strain CBDB1. Appl Environ Microbiol, 2007. 73(23): p. 7717-7724.

104. Padilla-Crespo, E., et al., Identification and environmental distribution of $d c p A$, which encodes the reductive dehalogenase catalyzing the dichloroelimination of 1,2-dichloropropane to propene in organohalide-respiring Chloroflexi. Appl Environ Microbiol, 2014. 80(3): p. 808-818.

105. Moe, W.M., et al., Dehalogenimonas lykanthroporepellens gen. nov., sp. nov., a reductively dehalogenating bacterium isolated from chlorinated solvent-contaminated groundwater. Int J Syst Evol Microbiol, 2009. 59(11): p. 2692-2697.

106. Yan, J., et al., Isolation of novel bacteria within the Chloroflexi capable of reductive dechlorination of 1,2,3 - trichloropropane. Environ Microbiol, 2009. 11(4): p. 833-843.

107. Siddaramappa, S., et al., Complete genome sequence of Dehalogenimonas lykanthroporepellens type strain (BL-DC-9T) and comparison to "Dehalococcoides" strains. Stand Genomic Sci, 2012. 6(2): p. 251 .

108. Mukherjee, K., et al., Dehalogenimonas lykanthroporepellens BL - DC - 9T simultaneously transcribes many rdhA genes during organohalide respiration with 1,2-DCA, 1,2-DCP and 1,2,3-TCP as electron acceptors. FEMS Microbiol lett, 2014. 351: p. 111-118.

109. Bowman, K.S., et al., Dehalogenimonas alkenigignens sp. nov., a chlorinated-alkane-dehalogenating bacterium isolated from groundwater. Int J Syst Evol Microbiol, 2013. 63(Pt 4): p. 1492-1498.

110. Wu, Q., K.R. Sowers, and H.D. May, Establishment of a polychlorinated biphenyl-dechlorinating microbial consortium, specific for doubly flanked chlorines, in a defined, sediment-free medium. Appl Environ Microbiol, 2000. 66(1): p. 49-53.

111. Wu, Q., et al., Identification of a bacterium that specifically catalyzes the reductive dechlorination of polychlorinated biphenyls with doubly flanked chlorines. Appl Environ Microbiol, 2002. 68(2): p. 807812.

112. Cutter, L.A., et al., Identification of a microorganism that links its growth to the reductive dechlorination of 2,3,5,6 - chlorobiphenyl. Environ Microbiol, 2001. 3(11): p. 699-709.

113. Fagervold, S.K., et al., Sequential reductive dechlorination of meta-chlorinated polychlorinated biphenyl congeners in sediment microcosms by two different Chloroflexi phylotypes. Appl Environ Microbiol, 2005. 71(12): p. 8085-8090.

114. Fagervold, S.K., H.D. May, and K.R. Sowers, Microbial reductive dechlorination of Aroclor 1260 in Baltimore Harbor sediment microcosms is catalyzed by three phylotypes within the phylum Chloroflexi. Appl Environ Microbiol, 2007. 73(9): p. 3009-3018.

115. Wu, Q., et al., Dechlorination of chlorobenzenes by a culture containing bacterium DF-1, a PCB dechlorinating microorganism. Environ Sci Technol, 2002. 36(15): p. 3290-3294.

116. Miller, G.S., et al., Reductive dechlorination of tetrachloroethene to trans-dichloroethene and cisdichloroethene by PCB-dechlorinating bacterium DF-1. Environ Sci Technol, 2005. 39(8): p. 26312635.

117. Kjellerup, B.V., et al., Spatial distribution of $P C B$ dechlorinating bacteria and activities in contaminated soil. Appl Environ Soil Sci, 2012. 2012: p. 584970.

118. Payne, R.B., H.D. May, and K.R. Sowers, Enhanced reductive dechlorination of polychlorinated biphenyl impacted sediment by bioaugmentation with a dehalorespiring bacterium. Environ Sci Technol, 2011. 45(20): p. 8772-8779.

119. Sanford, R.A., J.R. Cole, and J.M. Tiedje, Characterization and description of Anaeromyxobacter dehalogenans gen. nov., sp. nov., an aryl-halorespiring facultative anaerobic myxobacterium. Appl Environ Microbiol, 2002. 68(2): p. 893-900.

120. Cole, J.R., et al., Isolation and characterization of a novel bacterium growing via reductive dehalogenation of 2-chlorophenol. Appl Environ Microbiol, 1994. 60(10): p. 3536-3542.

121. Thomas, S.H., et al., The mosaic genome of Anaeromyxobacter dehalogenans strain 2CP-C suggests an aerobic common ancestor to the delta-Proteobacteria. PLoS One, 2008. 3(5): p. e2103.

122. Krumholz, L.R., Desulfuromonas chloroethenica sp. nov. uses tetrachloroethylene and trichloroethylene as electron acceptors. Int J Syst Bacteriol, 1997. 47(4): p. 1262-1263.

123. Krumholz, L.R., R. Sharp, and S.S. Fishbain, A freshwater anaerobe coupling acetate oxidation to tetrachloroethylene dehalogenation. Appl Environ Microbiol, 1996. 62(11): p. 4108-4113.

124. Rotaru, D.E.H., et al., Geobacter: the microbe electric's physiology, ecology, and practical applications Adv Microb Physiol, 2011. 59: p. 1. 
125. Lovley, D.R., et al., Geobacter metallireducens gen. nov. sp. nov., a microorganism capable of coupling the complete oxidation of organic compounds to the reduction of iron and other metals. Arch Microbiol, 1993. 159(4): p. 336-344.

126. De Wever, H., et al., Reductive dehalogenation of trichloroacetic acid by Trichlorobacter thiogenes gen. nov., sp. nov. Appl Environ Microbiol, 2000. 66(6): p. 2297-2301.

127. Sung, Y., et al., Geobacter lovleyi sp. nov. strain SZ, a novel metal-reducing and tetrachloroethenedechlorinating bacterium. Appl Environ Microbiol, 2006. 72(4): p. 2775-2782.

128. Wagner, D.D., et al., Genomic determinants of organohalide-respiration in Geobacter lovleyi, an unusual member of the Geobacteraceae. BMC Genomics, 2012. 13(1): p. 200.

129. Duhamel, M. and E.A. Edwards, Microbial composition of chlorinated ethene-degrading cultures dominated by Dehalococcoides. FEMS Microbiol Ecol, 2006. 58(3): p. 538-549.

130. Suzuki, D., et al., Desulfoluna butyratoxydans gen. nov., sp. nov., a novel Gram-negative, butyrateoxidizing, sulfate-reducing bacterium isolated from an estuarine sediment in Japan. Int J Syst Evol Microbiol, 2008. 58(4): p. 826-832.

131. Ahn, Y.-B., L.J. Kerkhof, and M.M. Häggblom, Desulfoluna spongiiphila sp. nov., a dehalogenating bacterium in the Desulfobacteraceae from the marine sponge Aplysina aerophoba. Int J Syst Evol Microbiol, 2009. 59(9): p. 2133-2139.

132. Ahn, Y.-B., et al., Reductive dehalogenation of brominated phenolic compounds by microorganisms associated with the marine sponge Aplysina aerophoba. Appl Environ Microbiol, 2003. 69(7): p. 41594166.

133. Sun, B., J.R. Cole, and J.M. Tiedje, Desulfomonile limimaris sp. nov., an anaerobic dehalogenating bacterium from marine sediments. Int J Syst Evol Microbiol, 2001. 51(2): p. 365-371.

134. Shelton, D.R. and J.M. Tiedje, Isolation and partial characterization of bacteria in an anaerobic consortium that mineralizes 3-chlorobenzoic acid. Appl Environ Microbiol, 1984. 48(4): p. 840-848.

135. Linkfield, T. and J. Tiedje, Characterization of the requirements and substrates for reductive dehalogenation by strain DCB-1. J Ind Microbiol, 1990. 5(1): p. 9-15.

136. DeWeerd, K., F. Concannon, and J.M. Suflita, Relationship between hydrogen consumption, dehalogenation, and the reduction of sulfur oxyanions by Desulfomonile tiedjei. Appl Environ Microbiol, 1991. 57(7): p. 1929-1934.

137. Boyle, A.W., C.D. Phelps, and L. Young, Isolation from estuarine sediments of a Desulfovibrio strain which can grow on lactate coupled to the reductive dehalogenation of 2,4,6-tribromophenol. Appl Environ Microbiol, 1999. 65(3): p. 1133-1140.

138. Sun, B., et al., Isolation and characterization of Desulfovibrio dechloracetivorans sp. nov., a marine dechlorinating bacterium growing by coupling the oxidation of acetate to the reductive dechlorination of 2-chlorophenol. Appl Environ Microbiol, 2000. 66(6): p. 2408-2413.

139. Baxter-Plant, V., et al., Dehalogenation of chlorinated aromatic compounds using a hybrid bioinorganic catalyst on cells of Desulfovibrio desulfuricans. Biotechnol Lett, 2004. 26(24): p. 1885 1890.

140. Schumacher, W., P.M. Kroneck, and N. Pfennig, Comparative systematic study on "Spirillum" 5175, Campylobacter and Wolinella species. Arch Microbiol, 1992. 158(4): p. 287-293.

141. Scholz-Muramatsu, H., et al., Isolation and characterization of Dehalospirillum multivorans gen. nov., sp. nov., a tetrachloroethene-utilizing, strictly anaerobic bacterium. Arch Microbiol, 1995. 163(1): p. 48-56.

142. Luijten, M.L., et al., Description of Sulfurospirillum halorespirans sp. nov., an anaerobic, tetrachloroethene-respiring bacterium, and transfer of Dehalospirillum multivorans to the genus Sulfurospirillum as Sulfurospirillum multivorans comb. nov. Int J Syst Evol Microbiol, 2003. 53(3): p. 787-793.

143. Holliger, C., G. Wohlfarth, and G. Diekert, Reductive dechlorination in the energy metabolism of anaerobic bacteria. FEMS Microbiol Rev, 1998. 22(5): p. 383-398.

144. Kräutler, B., et al., The cofactor of tetrachloroethene reductive dehalogenase of Dehalospirillum multivorans is norpseudo- $B_{12}$, a new type of a natural corrinoid. Helv Chim Acta, 2003. 86(11): p. 3698-3716.

145. Keller, S., et al., Exogenous 5,6-dimethylbenzimidazole caused production of a non-functional tetrachloroethene reductive dehalogenase in Sulfurospirillum multivorans. Environ Microbiol, 2014. 16(11): p. 3361-3369

146. Zhao, J.S., et al., Phylogeny of cyclic nitramine - degrading psychrophilic bacteria in marine sediment and their potential role in the natural attenuation of explosives. FEMS Microbiol Ecol, 2004. 49(3): p. 349-357. 
147. Zhao, J.-S., et al., Shewanella sediminis sp. nov., a novel $\mathrm{Na}^{+}$-requiring and hexahydro-1,3,5-trinitro1,3,5-triazine-degrading bacterium from marine sediment. Int J Syst Evol Microbiol, 2005. 55(4): p. 1511-1520.

148. Lohner, S.T. and A.M. Spormann, Identification of a reductive tetrachloroethene dehalogenase in Shewanella sediminis. Phil Trans R Soc B, 2013.368(1616): p. 20120326.

149. Cordova, C.D., et al., Partial functional replacement of CymA by SirCD in Shewanella oneidensis MR-1. J Bacteriol, 2011. 193(9): p. 2312-2321.

150. Zhao, J.-S., et al., Shewanella spp. genomic evolution for a cold marine lifestyle and in-situ explosive biodegradation. PLoS One, 2010. 5(2): p. e9109.

151. Chen, K., et al., Molecular characterization of the enzymes involved in the degradation of a brominated aromatic herbicide. Mol Microbiol, 2013. 89(6): p. 1121-1139.

152. Payne, K.A., et al., Reductive dehalogenase structure suggests a mechanism for B12-dependent dehalogenation. Nature, 2014. 517(7535): p. 513-516.

153. Lee, P.K.H., et al., Comparative genomics of two newly isolated Dehalococcoides strains and an enrichment using a genus microarray. ISME J, 2011. 5(6): p. 1014-1024.

154. Richardson, R.E., et al., Phylogenetic characterization of microbial communities that reductively dechlorinate TCE based upon a combination of molecular techniques. Environ Sci Technol, 2002. 36(12): p. 2652-2662.

155. Cheng, D., W.L. Chow, and J. He, A Dehalococcoides-containing co-culture that dechlorinates tetrachloroethene to trans-1,2-dichloroethene. ISME J, 2009. 4(1): p. 88-97.

156. Lee, P.K.H., et al., Isolation of two new Dehalococcoides mccartyi strains with dissimilar dechlorination functions and their characterization by comparative genomics via microarray analysis. Environ Microbiol, 2013. 15(8): p. 2293-2305.

157. He, J., et al., Complete detoxification of vinyl chloride by an anaerobic enrichment culture and identification of the reductively dechlorinating population as a Dehalococcoides species. Appl Environ Microbiol, 2003. 69(2): p. 996-1003.

158. Adrian, L., et al., Physiological characterization of a bacterial consortium reductively dechlorinating 1,2,3- and 1,2,4-trichlorobenzene. Appl Environ Microbiol, 1998. 64(2): p. 496-503.

159. Adrian, L., et al., Bacterial dehalorespiration with chlorinated benzenes. Nature, 2000. 408: p. $580-583$.

160. Bunge, M., et al., Enrichment of a dioxin-dehalogenating Dehalococcoides species in two-liquid phase cultures. Environ Microbiol, 2008. 10(10): p. 2670-2683.

161. He, J., et al., Isolation and characterization of Dehalococcoides sp. strain FL2, a trichloroethene (TCE)- and 1,2-dichloroethene-respiring anaerobe. Environ Microbiol, 2005. 7(9): p. 1442-1450.

162. Sung, Y., et al., Quantitative PCR confirms purity of strain GT, a novel trichloroethene-to-ethenerespiring Dehalococcoides isolate. Appl Environ Microbiol, 2006. 72(3): p. 1980-1987.

163. Rosner, B., P. McCarty, and A. Spormann, In vitro studies on reductive vinyl chloride dehalogenation by an anaerobic mixed culture. Appl Environ Microbiol, 1997. 63(11): p. 4139-4144.

164. Cheng, D. and J. He, Isolation and characterization of "Dehalococcoides" sp. strain MB, which dechlorinates tetrachloroethene to trans-1,2-dichloroethene. Appl Environ Microbiol, 2009. 75(18): p. 5910-5918.

165. Sanford, R.A., et al., Characterization of Desulfitobacterium chlororespirans sp. nov., which grows by coupling the oxidation of lactate to the reductive dechlorination of 3-chloro-4-hydroxybenzoat. Appl Environ Microbiol, 1996. 62(10): p. 3800-3808.

166. De Wildeman, S., et al., Stereoselective microbial dehalorespiration with vicinal dichlorinated alkanes. Appl Environ Microbiol, 2003. 69(9): p. 5643-5647.

167. Shelobolina, E.S., C.G. Vanpraagh, and D.R. Lovley, Use of ferric and ferrous iron containing minerals for respiration by Desulfitobacterium frappieri Geomicrobiol J, 2003. 20(2): p. 143-156.

168. Chang, Y.C., et al., Degradation of a variety of halogenated aliphatic compounds by an anaerobic mixed culture. J Ferment Bioeng, 1998. 86(4): p. 410-412.

169. Fletcher, K.E., et al., Resolution of culture Clostridium bifermentans DPH-1 into two populations, a Clostridium sp. and tetrachloroethene-dechlorinating Desulfitobacterium hafniense strain JH1. Appl Environ Microbiol, 2008. 74(19): p. 6141-6143.

170. Gerritse, J., et al., Influence of different electron donors and acceptors on dehalorespiration of tetrachloroethene by Desulfitobacterium frappieri TCE1. Appl Environ Microbiol, 1999. 65(12): p. 5212-5221.

171. Breitenstein, A., et al., Analysis of a 2,4,6-trichlorophenol-dehalogenating enrichment culture and isolation of the dehalogenating member Desulfitobacterium frappieri strain TCP-A. Arch Microbiol, 2001. 175: p. 133-142. 
172. Finneran, K.T., et al., Desulfitobacterium metallireducens sp. nov., an anaerobic bacterium that couples growth to the reduction of metals and humic acids as well as chlorinated compounds. Int J Syst Evol Microbiol, 2002. 52(6): p. 1929-1935.

173. Yoshida, N., et al., Isolation and quantitative detection of tetrachloroethene (PCE)-dechlorinating bacteria in unsaturated subsurface soils contaminated with chloroethenes. J Biosci Bioeng, 2007. 104(2): p. 91-97.

174. Gerritse, J., et al., Desulfitobacterium sp. strain PCE1, an anaerobic bacterium that can grow by reductive dechlorination of tetrachloroethene or ortho- chlorinated phenols. Arch Microbiol, 1996. 165 p. 132-140.

175. Miller, E., G. Wohlfarth, and G. Diekert, Comparative studies on tetrachloroethene reductive dechlorination mediated by Desulfitobacterium sp. strain PCE-S. Arch Microbiol, 1997. 168(6): p. 513-519.

176. Ding, C., S. Zhao, and J. He, A Desulfitobacterium sp. strain PR reductively dechlorinates both 1,1,1trichloroethane and chloroform. Environ Microbiol, 2014. 16(11): p. 3387-3397.

177. Löffler, F.E., K.M. Ritalahti, and J.M. Tiedje, Dechlorination of chloroethenes is inhibited by 2bromoethanesulfonate in the absence of methanogens. Appl Environ Microbiol, 1997. 63(12): p. 49824985.

178. Futagami, T., et al., Dehalogenation activities and distribution of reductive dehalogenase homologous genes in marine subsurface sediments. Appl Environ Microbiol, 2009. 75(21): p. 6905-6909.

179. Krzmarzick, M.J., et al., Natural niche for organohalide-respiring Chloroflexi. Appl Environ Microbiol, 2012. 78(2): p. 393-401.

180. Johnson, R.L., et al., Migration of chlorophenolic compounds at the chemical waste disposal site at Alkali Lake, Oregon - 2. Contaminant distributions, transport, and retardation. Groundwater, 1985. 23(5): p. 652-666.

181. Abelson, P.H., Inefficient remediation of ground-water pollution. Science, 1990. 250(4982).

182. Grøn, C., Organic halogens in Danish groundwaters. Lect Notes in Earth Sci, 1991. 33: p. 495-506.

183. Thornton, S.F., et al., Attenuation of landfill leachate by UK Triassic Sandstone aquifer materials: 2. Sorption and degradation of organic pollutants in laboratory columns. J Contam Hydrol, 2000. 43(3): p. 355-383.

184. Edmunds, W., et al., The natural (baseline) quality of groundwater: a UK pilot study. Sci Total Environ, 2003. 310(1): p. 25-35.

185. Emmanuel, E., M.G. Pierre, and Y. Perrodin, Groundwater contamination by microbiological and chemical substances released from hospital wastewater: Health risk assessment for drinking water consumers. Environ Int, 2009. 35(4): p. 718-726.

186. Löffler, F.E., et al., $16 S$ rRNA gene-based detection of tetrachloroethene-dechlorinating Desulfuromonas and Dehalococcoides species. Appl Environ Microbiol, 2000. 66(4): p. 1369-1374.

187. Fennell, D.E., et al., Assessment of indigenous reductive dechlorinating potential at a TCEcontaminated site using microcosms, polymerase chain reaction analysis, and site data. Environ Sci Technol, 2001. 35(9): p. 1830-1839.

188. Major, D.W., et al., Field demonstration of successful bioaugmentation to achieve dechlorination of tetrachloroethene to ethene. Environ Sci Technol, 2002. 36(23): p. 5106-5116.

189. Lendvay, J., et al., Bioreactive barriers: a comparison of bioaugmentation and biostimulation for chlorinated solvent remediation. Environ Sci Technol, 2003. 37(7): p. 1422-1431.

190. Nijenhuis, I., et al., Assessment of the natural attenuation of chlorinated ethenes in an anaerobic contaminated aquifer in the Bitterfeld/Wolfen area using stable isotope techniques, microcosm studies and molecular biomarkers. Chemosphere, 2007. 67(2): p. 300-311.

191. Rouzeau-Szynalski, K., J. Maillard, and C. Holliger, Frequent concomitant presence of Desulfitobacterium spp. and "Dehalococcoides" spp. in chloroethene-dechlorinating microbial communities. Appl Microbiol Biotechnol, 2011.90(1): p. 361-368.

192. Krajmalnik - Brown, R., et al., Environmental distribution of the trichloroethene reductive dehalogenase gene (tceA) suggests lateral gene transfer among Dehalococcoides. FEMS Microbiol Ecol, 2007. 59(1): p. 206-214.

193. McMurdie, P.J., et al., Site-specific mobilization of vinyl chloride respiration islands by a mechanism common in Dehalococcoides. BMC genomics, 2011. 12(1): p. 287.

194. Lee, P.K., et al., Quantifying genes and transcripts to assess the in situ physiology of "Dehalococcoides" spp. in a trichloroethene-contaminated groundwater site. Appl Environ Microbiol, 2008. 74(9): p. 2728-2739.

195. Ritalahti, K.M., et al., Quantitative PCR targeting $16 S$ rRNA and reductive dehalogenase genes simultaneously monitors multiple Dehalococcoides strains. Appl Environ Microbiol, 2006. 72(4): p. 2765-2774. 
196. Smits, T.H., et al., Development of a real-time PCR method for quantification of the three genera Dehalobacter, Dehalococcoides, and Desulfitobacterium in microbial communities. J Microbiol Methods, 2004. 57(3): p. 369-378.

197. Scheutz, C., et al., Concurrent ethene generation and growth of Dehalococcoides containing vinyl chloride reductive dehalogenase genes during an enhanced reductive dechlorination field demonstration. Environ Sci Technol, 2008. 42(24): p. 9302-9309.

198. Lookman, R., et al., Ground water transfer initiates complete reductive dechlorination in a PCE - contaminated aquifer. Ground Water Monit Remediat, 2007. 27(3): p. 65-74.

199. Schneidewind, U., et al., Kinetics of dechlorination by Dehalococcoides mccartyi using different carbon sources. J Contam Hydrol, 2014. 157: p. 25-36.

200. Courbet, C., et al., Complementing approaches to demonstrate chlorinated solvent biodegradation in a complex pollution plume: Mass balance, PCR and compound-specific stable isotope analysis. J Contam Hydrol, 2011. 126(3): p. 315-329.

201. van der Zaan, B., et al., Correlation of Dehalococcoides 16S rRNA and chloroethene-reductive dehalogenase genes with geochemical conditions in chloroethene-contaminated groundwater. Appl Environ Microbiol, 2010. 76(3): p. 843-850.

202. Damgaard, I., et al., Identification of chlorinated solvents degradation zones in clay till by high resolution chemical, microbial and compound specific isotope analysis. J Contam Hydrol, 2013. 146: p. 37-50.

203. Hug, L.A., et al., Community genomic analyses constrain the distribution of metabolic traits across the Chloroflexi phylum and indicate roles in sediment carbon cycling. Microbiome, 2013. 1(1): p. 22.

204. King, G.M., Dehalogenation in marine sediments containing natural sources of halophenols. Appl Environ Microbiol, 1988. 54(12): p. 3079-3085.

205. Boyle, A.W., M.M. Häggblom, and L.Y. Young, Dehalogenation of lindane ( $\gamma$-hexachlorocyclohexane) by anaerobic bacteria from marine sediments and by sulfate - reducing bacteria. FEMS Microbiol Ecol, 1999. 29(4): p. 379-387.

206. Berkaw, M., K.R. Sowers, and H.D. May, Anaerobic ortho dechlorination of polychlorinated biphenyls by estuarine sediments from Baltimore harbor. Appl Environ Microbiol, 1996. 62(7): p. 2534-2539.

207. Øfjord, G., J.A. Puhakka, and J.F. Ferguson, Reductive dechlorination of Aroclor 1254 by marine sediment cultures. Environ Sci Technol, 1994. 28(13): p. 2286-2294.

208. Watts, J.E., et al., A PCR-based specific assay reveals a population of bacteria within the Chloroflexi associated with the reductive dehalogenation of polychlorinated biphenyls. Microbiology, 2005. 151(6): p. 2039-2046.

209. Zanaroli, G., et al., A Chloroflexi bacterium dechlorinates polychlorinated biphenyls in marine sediments under in situ-like biogeochemical conditions. J Hazard Mater, 2012. 209: p. 449-457.

210. Ahn, Y.-B., M.M. Häggblom, and L.J. Kerkhof, Comparison of anaerobic microbial communities from estuarine sediments amended with halogenated compounds to enhance dechlorination of 1,2,3,4tetrachlorodibenzo-p-dioxin. FEMS Microbiol Ecol, 2007. 61(2): p. 362-371.

211. Inagaki, F., et al., Microbial communities associated with geological horizons in coastal subseafloor sediments from the Sea of Okhotsk. Appl Environ Microbiol, 2003. 69(12): p. 7224-7235.

212. Jorgensen, S.L., et al., Correlating microbial community profiles with geochemical data in highly stratified sediments from the Arctic Mid-Ocean Ridge. Proc Natl Acad Sci USA, 2012. 109(42): p. E2846-E2855.

213. Webster, G., et al., Prokaryotic community composition and biogeochemical processes in deep subseafloor sediments from the Peru Margin. FEMS Microbiol Ecol, 2006. 58(1): p. 65-85.

214. Wasmund, K., et al., Development and application of primers for the class Dehalococcoidia (phylum Chloroflexi) reveals highly diverse and stratified sub-group distributions in the marine subsurface. Environ Microbiol, 2015. 17(10): p. 3540-3556.

215. Futagami, T., et al., Distribution of dehalogenation activity in subseafloor sediments of the Nankai Trough subduction zone. Phil Trans R Soc B, 2013. 368(1616): p. 20120249.

216. Kawai, M., et al., High frequency of phylogenetically diverse reductive dehalogenase-homologous genes in deep subseafloor sedimentary metagenomes. Front Microbiol, 2014. 5: p. 80.

217. Suflita, J.M., et al., Dehalogenation: a novel pathway for the anaerobic biodegradation of haloaromatic compounds. Science, 1982. 218(4577): p. 1115-1117.

218. Horowitz, A., J.M. Suflita, and J.M. Tiedje, Reductive dehalogenations of halobenzoates by anaerobic lake sediment microorganisms. Appl Environ Microbiol, 1983. 45(5): p. 1459-1465.

219. Lorah, M.M. and L.D. Olsen, Natural attenuation of chlorinated volatile organic compounds in a freshwater tidal wetland: Field evidence of anaerobic biodegradation. Water Res, 1999. 35(12): p. 3811-3827. 
220. Lendvay, J.M., S.M. Dean, and P. Adriaens, Temporal and spatial trends in biogeochemical conditions at a groundwater-surface water interface: Implications for natural bioattenuation. Environ Sci Technol, 1998. 32(22): p. 3472-3478.

221. Conant, B., J.A. Cherry, and R.W. Gillham, A PCE groundwater plume discharging to a river: influence of the streambed and near-river zone on contaminant distributions. J Contam Hydrol, 2004. 73(1): p. 249-279.

222. Hamonts, K., et al., Factors determining the attenuation of chlorinated aliphatic hydrocarbons in eutrophic river sediment impacted by discharging polluted groundwater. Environ Sci Technol, 2009. 43(14): p. 5270-5275.

223. Abe, Y., et al., Evaluating the fate of chlorinated ethenes in streambed sediments by combining stable isotope, geochemical and microbial methods. J Contam Hydrol, 2009. 107(1): p. 10-21.

224. Hamonts, K., et al., Temporal variations in natural attenuation of chlorinated aliphatic hydrocarbons in eutrophic river sediments impacted by a contaminated groundwater plume. Water Res, 2012. 46(6): p. $1873-1888$.

225. Atashgahi, S., et al., Evaluation of solid polymeric organic materials for use in bioreactive sediment capping to stimulate the degradation of chlorinated aliphatic hydrocarbons. Appl Microbiol Biotechnol, 2014. 98(5): p. 2255-2266.

226. Atashgahi, S., et al., Small - scale oxygen distribution determines the vinyl chloride biodegradation pathway in surficial sediments of riverbed hyporheic zones. FEMS Microbiol Ecol, 2013. 84(1): p. 133142 .

227. Hamonts, K., et al., Determinants of the microbial community structure of eutrophic, hyporheic river sediments polluted with chlorinated aliphatic hydrocarbons. FEMS Microbiol Ecol, 2014. 87(3): p. 715-732.

228. Taş, N., et al., Tracking functional guilds: "Dehalococcoides" spp. in European river basins contaminated with hexachlorobenzene. Appl Environ Microbiol, 2009. 75(14): p. 4696-4704.

229. Kranzioch, I., et al., Dechlorination and organohalide-respiring bacteria dynamics in sediment samples of the Yangtze Three Gorges Reservoir. Environ Sci Poll R, 2013. 20(10): p. 7046-7056.

230. McNamara, P.J. and M.J. Krzmarzick, Triclosan enriches for Dehalococcoides-like Chloroflexi in anaerobic soil at environmentally relevant concentrations. FEMS Microbiol Lett, 2013. 344(1): p. 4852.

231. Delgado, A.G., et al., Selective enrichment yields robust ethene-producing dechlorinating cultures from microcosms stalled at cis-dichloroethene. PLoS One, 2014. 9(6): p. e100654.

232. Lanthier, M., et al., Geographic distribution of Desulfitobacterium frappieri PCP-1 and Desulfitobacterium spp. in soils from the province of Quebec, Canada. FEMS Microbiol Ecol, 2001. 36(2-3): p. 185-191.

233. Lévesque, M.-J., et al., Rapid method for detecting Desulfitobacterium frappieri strain PCP-1 in soil by the polymerase chain reaction. Appl Environ Microbiol, 1997. 47(6): p. 719-725.

234. Yoshida, N., et al., Polyphasic characterization of a PCP-to-phenol dechlorinating microbial community enriched from paddy soil. Sci Total Environ, 2007. 381(1): p. 233-242.

235. Krzmarzick, M.J., et al., Novel Firmicutes group implicated in the dechlorination of two chlorinated xanthones, analogues of natural organochlorines. Appl Environ Microbiol, 2014. 80(3): p. 1210-1218.

236. Rowe, A.R., et al., Characterization of the community structure of a dechlorinating mixed culture and comparisons of gene expression in planktonic and biofloc-associated "Dehalococcoides" and Methanospirillum species. Appl Environ Microbiol, 2008. 74(21): p. 6709-6719.

237. Hug, L.A., et al., Comparative metagenomics of three Dehalococcoides-containing enrichment cultures: the role of the non-dechlorinating community. BMC Genomics, 2012. 13: p. 327.

238. Stams, A.J. and C.M. Plugge, Electron transfer in syntrophic communities of anaerobic bacteria and archaea. Nat Rev Microbiol, 2009. 7(8): p. 568-577.

239. Fennell, D.E. and J.M. Gossett, Modeling the production of and competition for hydrogen in a dechlorinating culture. Environ Sci Technol, 1998. 32(16): p. 2450-2460.

240. Smatlak, C.R., J.M. Gossett, and S.H. Zinder, Comparative kinetics of hydrogen utilization for reductive dechlorination of tetrachloroethene and methanogenesis in an anaerobic enrichment culture. Environ Sci Technol, 1996. 30(9): p. 2850-2858.

241. Drzyzga, O., et al., Coexistence of a sulphate - reducing Desulfovibrio species and the dehalorespiring Desulfitobacterium frappieri TCE1 in defined chemostat cultures grown with various combinations of sulphate and tetrachloroethene. Environ microbiol, 2001. 3(2): p. 92-99.

242. Drzyzga, O. and J.C. Gottschal, Tetrachloroethene dehalorespiration and growth of Desulfitobacterium frappieri TCE1 in strict dependence on the activity of Desulfovibrio fructosivorans. Appl Environ Microbiol, 2002. 68(2): p. 642-649. 
243. Mägli, A., M. Wendt, and T. Leisinger, Isolation and characterization of Dehalobacterium formicoaceticum gen. nov. sp. nov., a strictly anaerobic bacterium utilizing dichloromethane as source of carbon and energy. Arch Microbiol, 1996. 166(2): p. 101-108.

244. Men, Y., et al., Characterization of four TCE-dechlorinating microbial enrichments grown with different cobalamin stress and methanogenic conditions. Appl Microbiol Biotechnol, 2013. 97: p. 1-12.

245. Shani, N., P. Rossi, and C. Holliger, Correlations between environmental variables and bacterial community structures suggest Fe (III) and vinyl chloride reduction as antagonistic terminal electronaccepting processes. Environ Sci Technol, 2013. 47(13): p. 6836-6845.

246. Cornelissen, G., et al., Extensive sorption of organic compounds to black carbon, coal, and kerogen in sediments and soils: mechanisms and consequences for distribution, bioaccumulation, and biodegradation. Environ Sci Technol, 2005. 39(18): p. 6881-6895.

247. Bunge, M. and U. Lechner, Anaerobic reductive dehalogenation of polychlorinated dioxins. Appl Microbiol Biotechnol, 2009. 84(3): p. 429-444.

248. Pöritz, M., et al., Genome sequences of two dehalogenation specialists-Dehalococcoides mccartyi strains BTF08 and DCMB5 enriched from the highly polluted Bitterfeld region. FEMS Microbiol lett, 2013. 343(2): p. 101-104.

249. Maillard, J., et al., Reductive dechlorination of tetrachloroethene by a stepwise catalysis of different organohalide respiring bacteria and reductive dehalogenases. Biodegradation, 2011. 22(5): p. 949-960.

250. van der Zee, F.P. and F.J. Cervantes, Impact and application of electron shuttles on the redox (bio) transformation of contaminants: a review. Biotechnol Adv, 2009. 27(3): p. 256-277.

251. Aulenta, F., et al., The humic acid analogue antraquinone-2, 6-disulfonate (AQDS) serves as an electron shuttle in the electricity-driven microbial dechlorination of trichloroethene to cisdichloroethene. Bioresour Technol, 2010. 101(24): p. 9728-9733.

252. Zhang, C. and A. Katayama, Humin as an electron mediator for microbial reductive dehalogenation. Environ Sci Technol, 2012. 46(12): p. 6575-6583.

253. Tobiszewski, M. and J. Namieśnik, Abiotic degradation of chlorinated ethanes and ethenes in water. Environ Sci Poll R, 2012. 19(6): p. 1994-2006.

254. Hosseinkhani, B., et al., Biogenic nanopalladium based remediation of chlorinated hydrocarbons in marine environments. Environ Sci Technol, 2014. 48(1): p. 550-557.

255. Ni, S., J.K. Fredrickson, and L. Xun, Purification and characterization of a novel 3-chlorobenzoatereductive dehalogenase from the cytoplasmic membrane of Desulfomonile tiedjei DCB-1. J Bacteriol, 1995. 177(17): p. 5135-5139.

256. Neumann, A., G. Wohlfarth, and G. Diekert, Purification and characterization of tetrachloroethene reductive dehalogenase from Dehalospirillum multivorans. J Biol Chem, 1996. 271(28): p. 1651516519.

257. Christiansen, N., et al., Purification and characterization of the 3-chloro-4-hydroxy-phenylacetate reductive dehalogenase of Desulfitobacterium hafniense. FEBS letters, 1998. 436(2): p. 159-162.

258. Okeke, B.C., et al., Purification, cloning, and sequencing of an enzyme mediating the reductive dechlorination of tetrachloroethylene (PCE) from Clostridium bifermentans DPH-1. Can J Microbiol, 2001. 47(5): p. 448-456.

259. Boyer, A., et al., Purification, cloning and sequencing of an enzyme mediating the reductive dechlorination of 2, 4, 6-trichlorophenol from Desulfitobacterium frappieri PCP-1. Biochem. J, 2003. 373: p. 297-303.

260. Neumann, A., G. Wohlfarth, and G. Diekert, Tetrachloroethene dehalogenase from Dehalospirillum multivorans: cloning, sequencing of the encoding genes, and expression of the pceA gene in Escherichia coli. J Bacteriol, 1998. 180(16): p. 4140-4145.

261. Kruse, T., et al., Complete genome sequence of Dehalobacter restrictus PER-K23 ${ }^{T}$. Stand Genomic Sci, 2013. 8(3): p. 375-88.

262. Berks, B.C., F. Sargent, and T. Palmer, The Tat protein export pathway. Mol Microbiol, 2000. 35(2): p. 260-274.

263. Edgar, R.C., MUSCLE: a multiple sequence alignment method with reduced time and space complexity. BMC bioinformatics, 2004. 5(1): p. 113.

264. Eddy, S.R., Profile hidden Markov models. Bioinformatics, 1998. 14(9): p. 755-763.

265. Stamatakis, A., RAxML-VI-HPC: maximum likelihood-based phylogenetic analyses with thousands of taxa and mixed models. Bioinformatics, 2006. 22(21): p. 2688-2690.

266. Stamatakis, A., P. Hoover, and J. Rougemont, A rapid bootstrap algorithm for the RAxML web servers. Syst Biol, 2008. 57(5): p. 758-771.

267. Letunic, I. and P. Bork, Interactive Tree Of Life (iTOL): an online tool for phylogenetic tree display and annotation. Bioinformatics, 2007. 23(1): p. 127-128. 
268. Letunic, I. and P. Bork, Interactive Tree Of Life v2: online annotation and display of phylogenetic trees made easy. Nucleic Acids Res, 2011: p. W475-478.

269. Tang, S. and E.A. Edwards, Identification of Dehalobacter reductive dehalogenases that catalyse dechlorination of chloroform, 1, 1, 1-trichloroethane and 1, 1-dichloroethane. Phil Trans R Soc B, 2013. 368(1616): p. 20120318.

270. Ding, C. and J. He, Molecular techniques in the biotechnological fight against halogenated compounds in anoxic environments. Microbial Biotechnol, 2012. 5(3): p. 347-367.

271. Regeard, C., J. Maillard, and C. Holliger, Development of degenerate and specific PCR primers for the detection and isolation of known and putative chloroethene reductive dehalogenase genes. J Microbiol Methods, 2004. 56(1): p. 107-118.

272. von Wintzingerode, F., et al., Development of primers for amplifying genes encoding CprA-and PceAlike reductive dehalogenases in anaerobic microbial consortia, dechlorinating trichlorobenzene and 1 , 2-dichloropropane. FEMS Microbiol Ecol, 2001. 35(2): p. 189-196.

273. Smidt, H., Molecular Characterization of Anaerobic Dehalogenation by Desulfitobacterium dehalogenans. PhD thesis, Wageningen University, The Netherlands, 2001.

274. Hölscher, T., et al., Multiple nonidentical reductive-dehalogenase-homologous genes are common in Dehalococcoides. Appl Environ Microbiol, 2004. 70(9): p. 5290-5297.

275. Thompson, J.D., D.G. Higgins, and T.J. Gibson, CLUSTAL W: improving the sensitivity of progressive multiple sequence alignment through sequence weighting, position-specific gap penalties and weight matrix choice. Nucleic Acids Res, 1994. 22(22): p. 4673-4680.

276. Grostern, A. and E.A. Edwards, Characterization of a Dehalobacter coculture that dechlorinates 1, 2dichloroethane to ethene and identification of the putative reductive dehalogenase gene. Appl Environ Microbiol, 2009. 75(9): p. 2684-2693.

277. Lohner, S.T. and A.M. Spormann, Identification of a reductive tetrachloroethene dehalogenase in Shewanella sediminis. Phil Trans R Soc B, 2013. 368(1616): p. 20120326.

278. Waller, A.S., et al., Multiple reductive-dehalogenase-homologous genes are simultaneously transcribed during dechlorination by Dehalococcoides-containing cultures. Appl Environ Microbiol, 2005. 71(12): p. 8257-8264.

279. Futamata, H., et al., Phylogenetic and transcriptional analyses of a tetrachloroethene-dechlorinating " Dehalococcoides" enrichment culture TUT2264 and its reductive-dehalogenase genes. Microbes Environ, 2009. 24(4): p. 330-337.

280. Marzorati, M., et al., Response of 1, 2-dichloroethane-adapted microbial communities to ex-situ biostimulation of polluted groundwater. Biodegradation, 2006. 17(2): p. 41-56.

281. Wagner, A., et al., Transcription analysis of genes encoding homologues of reductive dehalogenases in "Dehalococcoides" sp. strain CBDB1 by using terminal restriction fragment length polymorphism and quantitative PCR. Appl Environ Microbiol, 2009. 75(7): p. 1876-1884.

282. Fletcher, K.E., et al., Resolution of culture Clostridium bifermentans DPH-1 into two populations, a Clostridium sp. and tetrachloroethene-dechlorinating Desulfitobacterium hafniense strain JH1. Appl Environ Microbiol, 2008. 74(19): p. 6141-6143.

283. Rhee, S.K., et al., Detection by PCR of reductive dehalogenase motifs in a sulfidogenic 2-bromophenoldegrading consortium enriched from estuarine sediment. FEMS Microbiol Ecol, 2003. 43(3): p. $317-$ 324.

284. Kimoto, H., et al., Cloning of a novel dehalogenase from environmental DNA. Biosci Biotechnol Biochem, 2010. 74(6): p. 1290-1292.

285. Hug, L.A. and E.A. Edwards, Diversity of reductive dehalogenase genes from environmental samples and enrichment cultures identified with degenerate primer PCR screens. Front Microbiol, 2013. 4: p. 341.

286. Buttet, G.F., C. Holliger, and J. Maillard, Functional genotyping of Sulfurospirillum spp. in mixed cultures allowed the identification of a new tetrachloroethene reductive dehalogenase. Appl Environ Microbiol, 2013. 79(22): p. 6941-6947.

287. Maphosa, F., Chasing Organohalide Respirers: Ecogenomics Approaches to Assess the Bioremediation Capacity of Soils. PhD thesis, Wageningen University, The Netherlands, 2010.

288. Mayer-Blackwell, K., et al., Nanoliter qPCR Platform for Highly Parallel, Quantitative Assessment of Reductive Dehalogenase Genes and Populations of Dehalogenating Microorganisms in Complex Environments. Environ Sci Technol, 2014. 48: p. 9659-9667.

289. Johnson, D.R., et al., An internal reference technique for accurately quantifying specific mRNAs by real-time PCR with application to the tceA reductive dehalogenase gene. Appl Environ Microbiol, 2005. 71(7): p. 3866-3871. 
290. Holmes, V.F., et al., Discrimination of multiple Dehalococcoides strains in a trichloroethene enrichment by quantification of their reductive dehalogenase genes. Appl Environ Microbiol, 2006. 72(9): p. 5877-5883.

291. Maillard, J., C. Regeard, and C. Holliger, Isolation and characterization of Tn - Dha1, a transposon containing the tetrachloroethene reductive dehalogenase of Desulfitobacterium hafniense strain TCE1. Environ Microbiol, 2005. 7(1): p. 107-117.

292. Banerjee, R. and S.W. Ragsdale, The many faces of vitamin $B_{12}$ : catalysis by cobalamin-dependent enzymes. Annu Rev Biochem, 2003. 72: p. 209-47.

293. Zhang, Y., et al., Comparative genomic analyses of nickel, cobalt and vitamin $B_{12}$ utilization. BMC Genomics, 2009. 10: p. 78.

294. Moore, S.J. and M.J. Warren, The anaerobic biosynthesis of vitamin $B_{12}$. Biochem Soc Trans, 2012. 40(3): p. 581-6.

295. Scott, A.I., Discovering nature's diverse pathways to vitamin B B: a 35-year odyssey. J Org Chem, 2003. 68(7): p. 2529-39.

296. Schumacher, W., et al., Redox chemistry of cobalamin and iron-sulfur cofactors in the tetrachloroethene reductase of Dehalobacter restrictus. FEBS Lett, 1997. 409(3): p. 421-5.

297. Kräutler, B., et al., The cofactor of tetrachloroethene reductive dehalogenase of Dehalospirillum multivorans is norpseudo- $B_{12}$, a new type of a natural corrinoid. Helvetica Chimica Acta, 2003. 86(11): p. 3698-3716.

298. Reinhold, A., et al., Impact of Vitamin B12 on Formation of the Tetrachloroethene Reductive Dehalogenase in Desulfitobacterium hafniense Strain Y51. Appl Environ Microbiol, 2012. 78(22): p. 8025-32.

299. Yan, J., et al., Guided cobalamin biosynthesis supports Dehalococcoides mccartyi reductive dechlorination activity. Philos Trans R Soc Lond B Biol Sci, 2013. 368(1616): p. 20120320.

300. Men, Y., et al., Sustainable growth of Dehalococcoides mccartyi 195 by corrinoid salvaging and remodeling in defined lactate-fermenting consortia. Appl Environ Microbiol, 2014. 80(7): p. 2133-41.

301. Choudhary, P.K., et al., Diversity of cobalamin riboswitches in the corrinoid-producing organohalide respirer Desulfitobacterium hafniense. J Bacteriol, 2013. 195(22): p. 5186-5195.

302. Goris, T., et al., Insights into organohalide respiration and the versatile catabolism of Sulfurospirillum multivorans gained from comparative genomics and physiological studies. Environ Microbiol, 2014. 16(11): p. 3562-80.

303. Tang, S., Y. Gong, and E.A. Edwards, Semi-automatic in silico gap closure enabled de novo assembly of two Dehalobacter genomes from metagenomic data. PLoS One, 2012. 7(12): p. e52038.

304. Deshpande, N.P., et al., Genome sequence of Dehalobacter UNSWDHB, a chloroform-dechlorinating bacterium. Genome Announc, 2013. 1(5): p. e00720-13.

305. Altschul, S.F., et al., Basic local alignment search tool. J Mol Biol, 1990. 215(3): p. 403-10.

306. Larkin, M.A., et al., Clustal $W$ and Clustal X version 2.0. Bioinformatics, 2007. 23(21): p. $2947-8$.

307. Tamura, K., et al., MEGA5: molecular evolutionary genetics analysis using maximum likelihood, evolutionary distance, and maximum parsimony methods. Mol Biol Evol, 2011. 28(10): p. 2731-9.

308. Burge, S.W., et al., Rfam 11.0: 10 years of RNA families. Nucleic Acids Res, 2013. 41: p. D226-32.

309. Carver, T.J., et al., ACT: the Artemis Comparison Tool. Bioinformatics, 2005. 21(16): p. 3422-3.

310. Prat, L., et al., An unusual tandem-domain rhodanese harbouring two active sites identified in Desulfitobacterium hafniense. FEBS J, 2012. 279(15): p. 2754-67.

311. Sambrook, J., E.F. Fritsch, and T. Maniatis, Molecular cloning : a laboratory manual. 2nd ed. 1989, New York: Cold Spring Harbor Laboratory.

312. Rappsilber, J., M. Mann, and Y. Ishihama, Protocol for micro-purification, enrichment, prefractionation and storage of peptides for proteomics using StageTips. Nat Protoc, 2007. 2(8): p. 1896906.

313. Lu, J., et al., Filter-aided sample preparation with dimethyl labeling to identify and quantify milk fat globule membrane proteins. J Proteomics, 2011. 75(1): p. 34-43.

314. Cox, J. and M. Mann, MaxQuant enables high peptide identification rates, individualized p.p.b.-range mass accuracies and proteome-wide protein quantification. Nat Biotechnol, 2008. 26(12): p. 1367-72.

315. Rutherford, K., et al., Artemis: sequence visualization and annotation. Bioinformatics, 2000. 16(10): p. 944-5.

316. Smaczniak, C., et al., Proteomics-based identification of low-abundance signaling and regulatory protein complexes in native plant tissues. Nat Protoc, 2012. 7(12): p. 2144-58.

317. Fan, C. and T.A. Bobik, The PduX enzyme of Salmonella enterica is an L-threonine kinase used for coenzyme B12 synthesis. J Biol Chem, 2008. 283(17): p. 11322-9.

318. Poehlein, A., et al., An ancient pathway combining carbon dioxide fixation with the generation and utilization of a sodium ion gradient for ATP synthesis. PLoS One, 2012. 7(3): p. e33439. 
319. Nahvi, A., J.E. Barrick, and R.R. Breaker, Coenzyme $B_{12}$ riboswitches are widespread genetic control elements in prokaryotes. Nucleic Acids Res, 2004. 32(1): p. 143-50.

320. Schipp, C.J., et al., Organic cofactors in the metabolism of Dehalococcoides mccartyi strains. Philos Trans R Soc Lond B Biol Sci, 2013. 368(1616): p. 20120321.

321. Stupperich, E., H.J. Eisinger, and B. Kräutler, Diversity of corrinoids in acetogenic bacteria. Pcresolylcobamide from Sporomusa ovata, 5-methoxy-6-methylbenzimidazolylcobamide from Clostridium formicoaceticum and vitamin $B_{12}$ from Acetobacterium woodii. Eur J Biochem, 1988. 172(2): p. 459-64.

322. Ragsdale, S.W. and E. Pierce, Acetogenesis and the Wood-Ljungdahl pathway of $\mathrm{CO}_{2}$ fixation. Biochim Biophys Acta, 2008. 1784(12): p. 1873-98.

323. Johnson, D.R., et al., Transcriptomic microarray analysis of corrinoid responsive genes in Dehalococcoides ethenogenes strain 195. FEMS Microbiol Lett, 2009. 294(2): p. 198-206.

324. He, J., et al., Influence of vitamin B12 and cocultures on the growth of Dehalococcoides isolates in defined medium. Appl Environ Microbiol, 2007. 73(9): p. 2847-53.

325. Men, Y., et al., Sustainable syntrophic growth of Dehalococcoides ethenogenes strain 195 with Desulfovibrio vulgaris Hildenborough and Methanobacterium congolense: global transcriptomic and proteomic analyses. ISME J, 2012. 6(2): p. 410-21.

326. Barber, J.L., et al., Hexachlorobenzene in the global environment: Emissions, levels, distribution, trends and processes. Sci Total Environ, 2005. 349(1-3): p. 1-44.

327. Malcolm, H., P. Howe, and S. Dobson, Chlorobenzenes other than hexachlorobenzene: environmental aspects. 2004: World health organization.

328. Wang, G., et al., Hexachlorobenzene sources, levels and human exposure in the environment of China. Environ Int, 2010. 36(1): p. 122-130.

329. Jiang, L., et al., Influence of degradation behavior of coexisting chlorobenzene congeners pentachlorobenzene, 1, 2, 4, 5-tetrachlorobenzene, and 1, 2, 4-trichlorobenzene on the anaerobic reductive dechlorination of hexachlorobenzene in dye plant contaminated soil. Water Air Soil Pollut, 2015. 226(9): p. 1-9.

330. Ramanand, K., M. Balba, and J. Duffy, Reductive dehalogenation of chlorinated benzenes and toluenes under methanogenic conditions. Appl Environ Microbiol, 1993. 59(10): p. 3266-3272.

331. Taş, N., et al., Role of "Dehalococcoides" spp. in the anaerobic transformation of hexachlorobenzene in European rivers. Appl Environ Microbiol, 2011. 77(13): p. 4437-4445.

332. Taş, N., et al., Concurrent hexachlorobenzene and chloroethene transformation by endogenous dechlorinating microorganisms in the Ebro River sediment. FEMS Microbiol Ecol, 2010. 74(3): p. $682-$ 692.

333. Nowak, J., et al., Total reductive dechlorination of chlorobenzenes to benzene by a methanogenic mixed culture enriched from Saale river sediment. Appl Microbiol Biotechnol, 1996. 45(5): p. 700-709.

334. Fathepure, B.Z., J.M. Tiedje, and S.A. Boyd, Reductive dechlorination of hexachlorobenzene to tri-and dichlorobenzenes in anaerobic sewage sludge. Appl Environ Microbiol, 1988. 54(2): p. 327-330.

335. Fennell, D.E., et al., Dehalococcoides ethenogenes strain 195 reductively dechlorinates diverse chlorinated aromatic pollutants. Environ Sci Technol, 2004. 38(7): p. 2075-2081.

336. Zhou, X., et al., Polyphasic characterization of an anaerobic hexachlorobenzene-dechlorinating microbial consortium with a wide dechlorination spectrum for chlorobenzenes. J Biosci Bioeng, 2015. 120(1): p. 62-8.

337. Fung, J.M., et al., Reductive dehalogenation of dichlorobenzenes and monochlorobenzene to benzene in microcosms. Environ Sci Technol, 2009. 43(7): p. 2302-2307.

338. Adrian, L. and H. Görisch, Microbial transformation of chlorinated benzenes under anaerobic conditions. Res Microbiol, 2002. 153(3): p. 131-137.

339. Beurskens, J.E., et al., Dechlorination of chlorinated benzenes by an anaerobic microbial consortium that selectively mediates the thermodynamic most favorable reactions. Environ Sci Technol, 1994. 28(4): p. 701-706.

340. Vandermeeren, P., et al., Diversity of dechlorination pathways and organohalide respiring bacteria in chlorobenzene dechlorinating enrichment cultures originating from river sludge. Biodegradation, 2014: p. 1-20.

341. Yonezawa, Y., et al., Dechlorination of 1, 2, 4-trichlorobenzene in the sediment of Ise Bay. Chemosphere, 1994. 28(12): p. 2179-2184.

342. Masunaga, S., S. Susarla, and Y. Yonezawa, Dechlorination of chlorobenzenes in anaerobic estuarine sediment. Water Sci Technol, 1996. 33(6): p. 173-180.

343. Pavlostathis, S.G. and M.T. Prytula, Kinetics of the sequential microbial reductive dechlorination of hexachlorobenzene. Environ Sci Technol, 2000. 34(18): p. 4001-4009. 
344. Zanaroli, G., et al., Microbial dehalogenation of organohalides in marine and estuarine environments. Curr Opin Biotech, 2015. 33: p. 287-295.

345. Liang, X., et al., Pathway-dependent isotope fractionation during aerobic and anaerobic degradation of monochlorobenzene and 1, 2, 4-trichlorobenzene. Environ Sci Technol, 2011. 45(19): p. 8321-8327.

346. Ramiro-Garcia J, et al., NG - Tax, a highly accurate and validated pipeline for analysis of $16 \mathrm{~S} r \mathrm{RNA}$ amplicons from complex biomes. Submitted for publication, 2016.

347. Edgar, R.C., Search and clustering orders of magnitude faster than BLAST. Bioinformatics, 2010. 26(19): p. 2460-2461.

348. Quast, C., et al., The SILVA ribosomal RNA gene database project: improved data processing and webbased tools. Nucleic Acids Res, 2013. 41(D1): p. D590-D596.

349. Caporaso, J.G., et al., QIIME allows analysis of high-throughput community sequencing data. Nature Methods, 2010. 7(5): p. 335-336.

350. Tamura, K., et al., MEGA4: molecular evolutionary genetics analysis (MEGA) software version 4.0. Mol Biol Evol, 2007. 24(8): p. 1596-1599.

351. Lu, Y., et al., Effect of temperature on the structure and activity of a methanogenic archaeal community during rice straw decomposition. Soil Biol Biochem, 2015. 81: p. 17-27.

352. Sutton, N.B., et al., Microbial community response of an organohalide respiring enrichment culture to permanganate oxidation. PLoS One, 2015. 10(8): p. e0134615.

353. Dolfing, J. and B. Keith Harrison, Redox and reduction potentials as parameters to predict the degradation pathway of chlorinated benzenes in anaerobic environments. FEMS Microbiol Ecol, 1993. 13(1): p. 23-29.

354. Middeldorp, P., et al., Enrichment and properties of a 1, 2, 4-trichlorobenzene-dechlorinating methanogenic microbial consortium. Appl Environ Microbiol, 1997. 63(4): p. 1225-1229.

355. Duan, T.H. and L. Adrian, Enrichment of hexachlorobenzene and 1, 3, 5-trichlorobenzene transforming bacteria from sediments in Germany and Vietnam. Biodegradation, 2013. 24(4): p. 513-520.

356. Rahman, N.A., et al., A phylogenomic analysis of the bacterial phylum Fibrobacteres. Front Microbiol, 2015. 6: p. 1469.

357. Ronen, Z., et al., Metabolism of the explosive hexahydro-1, 3, 5-trinitro-1, 3, 5-triazine (RDX) in a contaminated vadose zone. Chemosphere, 2008. 73(9): p. 1492-1498.

358. Cupples, A.M., RDX degrading microbial communities and the prediction of microorganisms responsible for RDX bioremediation. Int Biodeterior Biodegradation, 2013. 85: p. 260-270.

359. Jeong, S., et al., Survival of introduced phosphate-solubilizing bacteria (PSB) and their impact on microbial community structure during the phytoextraction of Cd-contaminated soil. J Haz Mat, 2013. 263: p. 441-449.

360. Abbasian, F., et al., The biodiversity changes in the microbial population of soils contaminated with crude oil. Current Microbiol, 2016: p. 1-8.

361. Pérez-de-Mora, A., et al., Bioremediation of chlorinated ethenes in fractured bedrock and associated changes in dechlorinating and nondechlorinating microbial populations. Environ Sci Technol, 2014. 48(10): p. 5770-5779.

362. Grabowski, A., et al., Petrimonas sulfuriphila gen. nov., sp. nov., a mesophilic fermentative bacterium isolated from a biodegraded oil reservoir. Int J Syst Evol Microbiol, 2005. 55(3): p. 1113-1121.

363. Zhang, Y., et al., Trichloroethylene removal and bacterial variations in the up-flow anaerobic sludge blanket reactor in response to temperature shifts. Appl Microbiol Biotechnol, 2015. 99(14): p. 6091102.

364. Shivaji, S., et al., Sphingobacterium antarcticus sp. nov., a psychrotrophic bacterium from the soils of Schirmacher Oasis, Antarctica. Int J Syst Bacteriol, 1992. 42(1): p. 102-106.

365. Macbeth, T.W., et al., Molecular characterization of a dechlorinating community resulting from in situ biostimulation in a trichloroethene-contaminated deep, fractured basalt aquifer and comparison to a derivative laboratory culture. Appl Environ Microbiol, 2004. 70(12): p. 7329-7341.

366. Bunge, M., et al., Enrichment of a dioxin-dehalogenating Dehalococcoides species in two - liquid phase cultures. Environ Microbiol, 2008. 10(10): p. 2670-2683.

367. Livermore, J.A., et al., Microbial community dynamics during acetate biostimulation of RDXcontaminated groundwater. Environ Sci Technol, 2013. 47(14): p. 7672-7678.

368. Merlino, G., et al., Diverse reductive dehalogenases are associated with Clostridiales-enriched microcosms dechlorinating 1, 2-dichloroethane. BioMed Res Int, 2015. 2015: p. 242856.

369. Cichocka, D., et al., Tetrachloroethene conversion to ethene by a Dehalococcoides-containing enrichment culture from Bitterfeld. FEMS Microbiol Ecol, 2010. 72(2): p. 297-310.

370. Zhang, C., et al., Polyphasic characterization of two microbial consortia with wide dechlorination spectra for chlorophenols. J Biosci Bioeng, 2012. 114(5): p. 512-517. 
371. van den Bogert, B., et al., Microarray analysis and barcoded pyrosequencing provide consistent microbial profiles depending on the source of human intestinal samples. Appl Environ Microbiol, 2011. 77(6): p. 2071-2080.

372. Daims, H., et al., The domain-specific probe EUB338 is insufficient for the detection of all Bacteria: development and evaluation of a more comprehensive probe set. Syst Appl Microbiol, 1999. 22(3): p. 434-444.

373. Tian L, S.J., Borewicz K, van den Bogert B, Smidt H, Scheurink AJW, Gruppen H, Schols H Effects of pectin supplementation on the fermentation patterns of different structural carbohydrates in rats. Under revision, 2016.

374. Stroo, H.F., et al., Chlorinated ethene source remediation: Lessons learned. Environ Sci Technol, 2012. 46(12): p. 6438-6447.

375. Kielhorn, J., et al., Vinyl chloride: Still a cause for concern. Environ Health Persp, 2000. 108(7): p. 579-588.

376. Löffler, F.E. and E.A. Edwards, Harnessing microbial activities for environmental cleanup. Curr Opin Biotechnol, 2006. 17(3): p. 274-284.

377. Ellis, D.E., et al., Bioaugmentation for accelerated in situ anaerobic bioremediation. Environ Sci Technol, 2000. 34(11): p. 2254-2260.

378. Atashgahi, S., Y. Lu, and H. Smidt, Overview of known organohalide-respiring bacteria-phylogenetic diversity and environmental distribution, in Organohalide respiring bacteria. Accepted for publication. 2016.

379. Damgaard, I., et al., Performance of Full-Scale Enhanced Reductive Dechlorination in Clay Till. Groundwater Monit Remediat, 2013. 33(1): p. 48-61.

380. Révész, K.M., et al., Integration of stable carbon isotope, microbial community, dissolved hydrogen gas, and ${ }^{2} \mathrm{H}_{\mathrm{H} 2 \mathrm{O}}$ tracer data to assess bioaugmentation for chlorinated ethene degradation in fractured rocks. J Contam Hydrol, 2014. 156: p. 62-77.

381. Ritalahti, K.M., et al., Comparing on-site to off-site biomass collection for Dehalococcoides biomarker gene quantification to predict in situ chlorinated ethene detoxification potential. Environ Sci Technol, 2010. 44(13): p. 5127-5133.

382. Sutton, N.B., et al., Microbial dynamics during and after in situ chemical oxidation of chlorinated solvents. Groundwater, 2015. 53(2): p. 261-270.

383. Rahm, B.G., et al., Molecular characterization of microbial populations at two sites with differing reductive dechlorination abilities. Biodegradation, 2006. 17(6): p. 523-534.

384. Lee, P.K.H., et al., Phylogenetic microarray analysis of a microbial community performing reductive dechlorination at a TCE-contaminated site. Environ Sci Technol, 2012. 46(2): p. 1044-1054.

385. Bowman, K.S., et al., Bacterial diversity of an acidic Louisiana groundwater contaminated by dense nonaqueous-phase liquid containing chloroethanes and other solvents. FEMS Microbiol Ecol, 2006. 58(1): p. 120-133.

386. Dojka, M.A., et al., Microbial diversity in a hydrocarbon- and chlorinated-solvent- contaminated aquifer undergoing intrinsic bioremediation. Appl Environ Microbiol, 1998. 64(10): p. 3869-3877.

387. Hohnstock-Ashe, A.M., et al., Further biogeochemical characterization of a trichloroethenecontaminated fractured dolomite aquifer: Electron source and microbial communities involved in reductive dechlorination. Environ Sci Technol, 2001. 35(22): p. 4449-4456.

388. Imfeld, G., et al., Assessment of in situ degradation of chlorinated ethenes and bacterial community structure in a complex contaminated groundwater system. Water Res, 2008. 42(4-5): p. 871-882.

389. Lowe, M., et al., Geochemistry and microbial diversity of a trichloroethene-contaminated Superfund site undergoing intrinsic in situ reductive dechlorination. FEMS Microbiol Ecol, 2002. 40(2): p. 123134.

390. Rossi, P., et al., Ecology and biogeography of bacterial communities associated with chloroethenecontaminated aquifers. Front Microbiol, 2012. 3: p. 260.

391. Imfeld, G., et al., Characterization of groundwater microbial communities, dechlorinating bacteria, and in situ biodegradation of chloroethenes along a vertical gradient. Water Air Soil Pollut, 2011. 221(1-4): p. 107-122.

392. Shade, A., et al., Fundamentals of microbial community resistance and resilience. Front Microbiol, 2012. 3: p. 417.

393. Aeppli, C., et al., Quantifying in situ transformation rates of chlorinated ethenes by combining compound-specific stable isotope analysis, groundwater dating, and carbon isotope mass balances. Environ Sci Technol, 2010. 44(10): p. 3705-3711.

394. Anderson, M.J., A new method for non-parametric multivariate analysis of variance. Austral Ecol, 2001. 26(1): p. 32-46. 
395. Clarke, K.R., Non-parametric multivariate analyses of changes in community structure. Aust J Ecol, 1993. 18(1): p. 117-143.

396. Jones, D., The Fathom Toolbox for MATLAB: software for multivariate ecological and oceanographic data analysis. College of Marine Science, University of South Florida, Tampa, FL, USA. Available from: http://www.marine.usf.edu/user/djones/. 2015.

397. Dejean S, G.I., Lê Cao K-A, Monget P., mixOmics: Omics data integration project. In: $R$ Package version. Available: http://cran.r-project.org/. 2011: p. 2-9.

398. Lê Cao, K.A., I. González, and S. Déjean, IntegrOmics: An R package to unravel relationships between two omics datasets. Bioinformatics, 2009. 25(21): p. 2855-2856.

399. Hunkeler, D., et al., A guide for assessing biodegradation and source identification of organic ground water contaminants using compound specific isotope analysis (CSIA). EPA 600/R-08/148. 2008: Office of Research and Development, National Risk Management Research Laboratory, US Environmental Protection Agency.

400. Dugat-Bony, E., et al., In situ TCE degradation mediated by complex dehalorespiring communities during biostimulation processes. Microbial Biotechnol, 2012. 5(5): p. 642-653.

401. Campbell, B.J., et al., The versatile e-proteobacteria: Key players in sulphidic habitats. Nature Rev Microbiol, 2006. 4(6): p. 458-468.

402. Canfield, D.E., The evolution of the Earth surface sulfur reservoir. Am J Sci, 2004. 304(10): p. 839-861.

403. Kodama, Y. and K. Watanabe, Sulfuricurvum kujiense gen. nov., sp. nov., a facultatively anaerobic, chemolithoautotrophic, sulfur-oxidizing bacterium isolated from an underground crude-oil storage cavity. Int J Syst Evol Microbiol, 2004. 54(6): p. 2297-2300.

404. Kao, C.M., et al., The change of microbial community from chlorinated solvent-contaminated groundwater after biostimulation using the metagenome analysis. J Haz Mat, 2016. 302: p. 144-150.

405. Tsai, T.T., et al., Application of polycolloid-releasing substrate to remediate trichloroethylenecontaminated groundwater: A pilot-scale study. J Haz Mat, 2014. 268: p. 92-101.

406. Bernardet, J.F. and P.A.D. Grimont, Deoxyribonucleic acid relatedness and phenotypic characterization of Flexibacter columnaris sp. nov., nom. rev., Flexibacter psychrophilus sp. nov., nom. rev., and Flexibacter maritimus Wakabayashi, Hikida, and Masumura 1986. Int J Syst Bacteriol, 1989. 39(3): p. 346-354.

407. Bernardet, J.F., et al., Cutting a gordian knot: Emended classification and description of the genus Flavobacterium, emended description of the family Flavobacteriaceae, and proposal of Flavobacterium hydatis nom. nov. (basonym, Cytophaga aquatilis Strohl and Tait 1978). Int J Syst Bacteriol, 1996. 46(1): p. 128-148.

408. Biebl, H., Fermentation of glycerol by Clostridium pasteurianum - Batch and continuous culture studies. J Ind Microbiol Biotechnol, 2001. 27(1): p. 18-26.

409. Moe, W.M., et al., Pelosinus defluvii sp. nov., isolated from chlorinated solvent-contaminated groundwater, emended description of the genus Pelosinus and transfer of Sporotalea propionica to Pelosinus propionicus comb. nov. Int J Syst Evol Microbiol, 2012. 62(6): p. 1369-1376.

410. van Gelder, A.H., et al., 1,3-Propanediol production from glycerol by a newly isolated Trichococcus strain. Microbial Biotechnol, 2012. 5(4): p. 573-578.

411. Wilkens, E., et al., High-level production of 1,3-propanediol from crude glycerol by Clostridium butyricum AKR102a. Appl Environ Microbiol, 2012.93(3): p. 1057-1063.

412. Rabus, R., T.A. Hansen, and F. Widdel, Dissimilatory sulfate- and sulfur-reducing prokaryotes, in In The Prokaryotes: Prokaryotic Physiology and Biochemistry. 2013, Springer. p. 309-404.

413. Qatibi, A.I., et al., Anaerobic degradation of glycerol by Desulfovibrio fructosovorans and D. carbinolicus and evidence for glycerol-dependent utilization of 1,2-propanediol. Current Microbiol, 1998. 36(5): p. 283-290.

414. Qatibi, A.I., A. Bories, and J.L. Garcia, Sulfate reduction and anaerobic glycerol degradation by a mixed microbial culture. Current Microbiol, 1991. 22(1): p. 47-52.

415. Hoelen, T. and M. Reinhard, Complete biological dehalogenation of chlorinated ethylenes in sulfate containing groundwater. Biodegradation, 2004. 15(6): p. 395-403.

416. Zhou, J., et al., Stochasticity, succession, and environmental perturbations in a fluidic ecosystem. Proc Natl Acad Sci USA, 2014. 111(9): p. E836-E845.

417. Handley, K.M., et al., Biostimulation induces syntrophic interactions that impact $C$, $S$ and $N$ cycling in a sediment microbial community. ISME J, 2013. 7(4): p. 800-816.

418. Briée, C., D. Moreira, and P. López-García, Archaeal and bacterial community composition of sediment and plankton from a suboxic freshwater pond. Res Microbiol, 2007. 158(3): p. 213-227.

419. Elshahed, M.S., et al., Metagenomic analysis of the microbial community at Zodletone Spring (Oklahoma): Insights into the genome of a member of the novel candidate division OD1. Appl Environ Microbiol, 2005. 71(11): p. 7598-7602. 
420. Peura, S., et al., Distinct and diverse anaerobic bacterial communities in boreal lakes dominated by candidate division OD1. ISME J, 2012. 6(9): p. 1640-1652.

421. Gihring, T.M., et al., A limited microbial consortium is responsible for extended bioreduction of uranium in a contaminated aquifer. Appl Environ Microbiol, 2011. 77(17): p. 5955-5965.

422. Wrighton, K.C., et al., Fermentation, hydrogen, and sulfur metabolism in multiple uncultivated bacterial phyla. Science, 2012. 337(6102): p. 1661-1665.

423. Wrighton, K.C., et al., Metabolic interdependencies between phylogenetically novel fermenters and respiratory organisms in an unconfined aquifer. ISME J, 2014. 8(7): p. 1452-1463.

424. Dennis, P.C., et al., Phylogenetic analysis of bacterial populations in an anaerobic microbial consortium capable of degrading saturation concentrations of tetrachloroethylene. Can J Microbiol, 2003. 49(1): p. 15-27.

425. Duhamel, M., K. Mo, and E.A. Edwards, Characterization of a highly enriched Dehalococcoidescontaining culture that grows on vinyl chloride and trichloroethene. Appl Environ Microbiol, 2004. 70(9): p. 5538-5545.

426. Gu, A.Z., et al., Analysis and comparison of the microbial community structures of two enrichment cultures capable of reductively dechlorinating TCE and cis-DCE. Environ Microbiol, 2004. 6(1): p. 4554.

427. Yang, Y., et al., Identification of microorganisms involved in reductive dehalogenation of chlorinated ethenes in an anaerobic microbial community. Water Res, 2005. 39(16): p. 3954-3966.

428. Daprato, R.C., F.E. Löffler, and J.B. Hughes, Comparative analysis of three tetrachloroethene to ethene halorespiring consortia suggests functional redundancy. Environ Sci Technol, 2007. 41(7): p. 22612269.

429. Freeborn, R.A., et al., Phylogenetic analysis of TCE-dechlorinating consortia enriched on a variety of electron donors. Environ Sci Technol, 2005. 39(21): p. 8358-8368.

430. van den Bogert, B., et al., Microarray analysis and barcoded pyrosequencing provide consistent microbial profiles depending on the source of human intestinal samples. Appl Environ Microbiol, 2011. 77(6): p. 2071-2080.

431. Daims, H., et al., The domain-specific probe EUB338 is insufficient for the detection of all bacteria: Development and evaluation of a more comprehensive probe set. Syst Appl Microbiol, 1999. 22(3): p. 434-444.

432. Lovelock, J., Natural halocarbons in the air and in the sea. Nature, 1975. 256: p. 193-194.

433. Biderre-Petit, C., et al., Distribution of Dehalococcoidia in the Anaerobic Deep Water of a Remote Meromictic Crater Lake and Detection of Dehalococcoidia-Derived Reductive Dehalogenase Homologous Genes. PLoS One, 2016. 11(1): p. e0145558.

434. Weissflog, L., et al., Sediments of salt lakes as a new source of volatile highly chlorinated C1/C2 hydrocarbons. Geophys Res Lett, 2005. 32(1): p. L01401.

435. Rücker, A., et al., Halogenated hydrocarbon formation in a moderately acidic salt lake in Western Australia-role of abiotic and biotic processes. Environ Chem, 2015. 12(4): p. 406-414.

436. Dyall-Smith, M., The Halohandbook: protocols for haloarchaeal genetics. Available from: http://www.haloarchaea.com/resources/halohandbook/index.html. 2009.

437. Rhew, R.C., B.R. Miller, and R.F. Weiss, Natural methyl bromide and methyl chloride emissions from coastal salt marshes. Nature, 2000. 403(6767): p. 292-295.

438. Rhew, R. and O. Mazéas, Gross production exceeds gross consumption of methyl halides in northern California salt marshes. Geophys Res Lett, 2010.37(18).

439. Kotte, K., et al., Organohalogen emissions from saline environments-spatial extrapolation using remote sensing as most promising tool. Biogeosciences, 2012. 9(3): p. 1225-1235.

440. Weigold, P., et al., Ribosomal tag pyrosequencing of DNA and RNA reveals 'rare'taxa with high protein synthesis potential in the sediment of a hypersaline lake in Western Australia. Geomicrobiol J, 2015. 0: p. 1-15.

441. Mebius, L.J., A rapid method for the determination of organic carbon in soil. Anal Chim Acta, 1960. 22 p. $120-124$

442. Amstaetter, K., T. Borch, and A. Kappler, Influence of humic acid imposed changes of ferrihydrite aggregation on microbial Fe (III) reduction. Geochim Cosmochim Acta, 2012. 85: p. 326-341.

443. Emmerich, M., et al., Abundance, distribution, and activity of Fe (II)-oxidizing and Fe (III)-reducing microorganisms in hypersaline sediments of Lake Kasin, southern Russia. Appl Environ Microbiol, 2012. 78(12): p. 4386-4399.

444. Team, R.C., R: A language and environment for statistical computing. Available from: https://www.rproject.org/. 2014. 
445. Muyzer, G., E.C. De Waal, and A.G. Uitterlinden, Profiling of complex microbial populations by denaturing gradient gel electrophoresis analysis of polymerase chain reaction-amplified genes coding for 16S rRNA. Appl Environ Microbiol, 1993. 59(3): p. 695-700.

446. Yu, Y., et al., Group - specific primer and probe sets to detect methanogenic communities using quantitative real - time polymerase chain reaction. Biotechnol Bioeng, 2005. 89(6): p. 670-679.

447. Amos, B.K., et al., Detection and quantification of Geobacter lovleyi strain SZ: implications for bioremediation at tetrachloroethene-and uranium-impacted sites. Appl Environ Microbiol, 2007. 73(21): p. 6898-6904.

448. Azizian, M.F., et al., Comparison of lactate, formate, and propionate as hydrogen donors for the reductive dehalogenation of trichloroethene in a continuous-flow column. J Cont Hydrol, 2010. 113(1): p. 77-92.

449. Uchino, Y., et al., Complete genome sequencing of Dehalococcoides sp. strain UCH007 using a differential reads picking method. Stand Genomic Sci, 2015. 10(1): p. 1.

450. Wong, Y.K., et al., Isolation and characterization of Dehalobacter sp. strain UNSWDHB capable of chloroform and chlorinated ethane respiration. Environ Microbiol, 2016.

451. Nichols, D., et al., Use of ichip for high-throughput in situ cultivation of "uncultivable" microbial species. Appl Environ Microbiol, 2010. 76(8): p. 2445-2450.

452. Ingham, C.J., et al., The micro-Petri dish, a million-well growth chip for the culture and highthroughput screening of microorganisms. Proc Natl Acad Sci USA, 2007. 104(46): p. 18217-18222.

453. Fullerton, H. and C.L. Moyer, Comparative single-cell genomics of Chloroflexi from the Okinawa Trough deep-subsurface biosphere. Appl Environ Microbiol, 2016. 82(10): p. 3000-3008.

454. Bommer, M., et al., Structural basis for organohalide respiration. Science, 2014. 346(6208): p. 455458.

455. Yang, C., et al., Reductive dehalogenation of oligocyclic phenolic bromoaromatics by Dehalococcoides mccartyi Strain CBDB1. Environ Sci Technol, 2015. 49(14): p. 8497-8505.

456. Dolfing, J., Thermodynamic considerations for dehalogenation, in Dehalogenation: microbial processes and environmental applications. 2003, Springer. p. 89-114.

457. Simon, C. and R. Daniel, Metagenomic analyses: past and future trends. Appl Environ Microbiol, 2011. 77(4): p. 1153-1161.

458. de Menezes, A., N. Clipson, and E. Doyle, Comparative metatranscriptomics reveals widespread community responses during phenanthrene degradation in soil. Environ Microbiol, 2012. 14(9): p. 2577-2588.

459. Bastida, F., et al., Soil metaproteomics: a review of an emerging environmental science. Significance, methodology and perspectives. Eur J Soil Sci, 2009. 60(6): p. 845-859.

460. Maron, P.-A., et al., Metaproteomics: a new approach for studying functional microbial ecology. Microb Ecol, 2007. 53(3): p. 486-493.

461. Mao, X., et al., Efficient metabolic exchange and electron transfer within a syntrophic TCE degrading co-culture of Dehalococcoides mccartyi 195 and Syntrophomonas wolfei. Appl Environ Microbiol, 2015. 81: p. 2015-2024.

462. Adriaens, P., et al., Bacterial dehalogenation of chlorobenzoates and coculture biodegradation of 4, 4'dichlorobiphenyl. Appl Environ Microbiol, 1989. 55(4): p. 887-892.

463. Krzmarzick, M.J., et al., Abundance and diversity of organohalide-respiring bacteria in lake sediments across a geographical sulfur gradient. FEMS Microbiol Ecol, 2013. 84(2): p. 248-258.

464. François Thomas, et al., Environmental and gut bacteroidetes: the food connection. Front Microbiol, 2011. 2: p. 93.

465. Megharaj, M., et al., Bioremediation approaches for organic pollutants: a critical perspective. Environ Int, 2011. 37(8): p. 1362-1375.

466. Lefebvre, O., et al., Treatment of hypersaline industrial wastewater by a microbial consortium in a sequencing batch reactor. Environ Technol, 2004. 25(5): p. 543-553. 


\section{Summary}

Many halogenated organic compounds (organohalides) are known environmental pollutants, threatening the health of humans and other animals. To address the environmental problems caused by organohalide pollution, successful application of bioremediation with efficient microbes is essential. Organohalide-respiring bacteria (OHRB) are able to breathe organohalides persistent in a broad range of oxygen-depleted environments and therefore present considerable potential for organohalide bioremediation. Nevertheless, to assess and adjust in situ bioremediation strategies, thorough understanding of ecophysiology of OHRB and their interaction with surrounding biotic and abiotic forces is necessary. To this end, this thesis focused on exploring ecophysiology and environmental distribution of OHRB and unraveling their interactions with the co-existing microbial guilds in the community and geochemical parameters by application of a suite of physiological, molecular and geochemical analyses.

Firstly, we provided a comprehensive overview of currently known OHRB isolates and highlighted their environmental distribution and factors impacting it (Chapter 2). In contrast to the limitted/distinct locations sampled for isolation, no restriction for the geographical distribution of OHRB was observed in molecular surveys. The important environmental factors that affect the distribution and activity of OHRB were discussed, and chasing novel OHRB from extreme environments was proposed as the known OHRB isolates survive/thrive in moderate $\mathrm{pH}$ and salinity range in laboratory culture. Therefore, in Chapter 7, with the organohalide respiration (OHR) potential was surveyed in hypersaline sediments collected from Lake Strawbridge, Western Australia, to assess the presence of OHRB. As a result, for the first time, the dechlorination of chloroform and perchloroethene (PCE) to dichloromethane and trichloroethene was documented from an alkaline hypersaline pristine environment. Combined with former report on biological production of organohalides at that site, Chapter 7 provided intriguing evidence of potentially interdependent biological chlorination and dechlorination in local halogen cycles occurring in hypersaline lakes.

Most molecular surveys for the presence and functionality of OHRB employ primer-based detection of their 16S rRNA and reductive dehalogenase (RDase) encoding genes. A comprehensive overview of degenerate/specific primers designed to target RDase encoding genes and primer-dependent techniques applied to date was provided Chapter 3. Further, the current trend in application of primer-independent, high-throughput approaches to expand our 
knowledge of the OHRB and their RDase genes pool was discussed. Due to pros and cons associated with primer-(in)dependent methods, a combined application is more advantages.

Dehalobacter restrictus PER-K23 ${ }^{\mathrm{T}}$ was applied as a model strain to study corrinoid auxotrophy in Chapter 4. Corrinoid at fifty $\mu \mathrm{g} / \mathrm{L}$ was found essential for sufficient PCE dechlorination. Transcriptional analysis and shotgun proteomics confirmed that the cobalamin biosynthesis pathway in $D$. restrictus is non-functional due to a 101-bp truncation in the cbiH gene, and suggested up-regulation of relevant cobalamin salvaging and transport pathways to ensure sufficient corrinoid supply under partial corrinoid starvation.

The importance of syntrophic interactions to support robust growth of OHRB was described in Chapters 5\&6. In Chapter 5, bacteria belonging to the Bacteroidetes phylum and Clostridiales order as well as sulfate-reducing members of Deltaproteobacteria class were suggested as putative stimulating guilds that provide electron donor and/or organic cofactors to chlorinated benezenes dechlorinators, i.e. D. mccartyi and Dehalobacter, in sediment microcosms and transferred cultures derived from contaminated harbor sludge. In Chapter 6, a similar syntrophic interaction between $D$. mccartyi and fermenting and/or sulfate reducing bacteria were noticed for robust dechlorination of chlorinated ethenes after in situ biostimulation by glycerol injection. Furthermore, the appearance of Epsilonproteobacteria and Deferribacteres as putative oxidizers of reduced sulfur compounds in the late stage of the filed study suggested that on one hand they might serve as detoxifiers of sulfide stimulating $D$. mccartyi, but on the other hand could be inhibitory as successors of the more important syntrophs belonging to fermenters and sulfate reducers.

In conclusion, this thesis expanded our understanding of ecophysiology and environmental distribution of OHRB, addressing their presence in pristine environments as well as providing further evidence for their dependencies on other microbial community members in order to meet their nutritional requirements. Hence, research described here contributes to increasing our knowledge as a foundation for evaluating and optimizing strategies for the bioremediation of organohalide contaminated sites. 


\section{Appendices}

Nederlandse samenvatting $\quad 221$

Acknowledgments 225

About the author 231

List of publication 233

$\begin{array}{ll}\text { Co-author affiliations } & 235\end{array}$

$\begin{array}{ll}\text { SENSE Diploma } & 237\end{array}$ 


\section{Nederlandse samenvatting}

Veel organische halogeenverbindingen zijn vervuilend voor het milieu en gevaarlijk voor de gezondheid van mensen en dieren. Om de problemen van vervuiling met organische halogeenverbindingen te overkomen, is het essentieel dat er succesvol bioremediatie met efficiënte microben wordt toegepast. Organische halogeenverbinding-gebruikende bacteriën (OHGB) kunnen deze verbindingen gebruiken als elektronen acceptor in een groot scala aan zuurstof arme milieus en kunnen daardoor worden ingezet voor bioremediatie. Echter, voor een optimalisatie van bioremediatie strategieën moet er nog beter begrip komen van de ecofysiologie van OHGB en de interactie met biotische en a-biotische factoren. Om dit te bereiken wordt in dit proefschrift de ecofysiologie en de verspreiding van OHGB onderzocht en hun interactie en samenleving met de lokale microbiële gemeenschap en geochemische parameters. Dit wordt onderzocht met behulp van een scala aan fysiologische, moleculaire en geochemische benaderingen.

Om te beginnen, hebben wij een uitgebreid overzicht gemaakt van de huidige bekende OHGB isolaten en hebben daarbij de nadruk gelegd op hun verspreiding in het milieu en de effecten die dat heeft (Hoofdstuk 2). Ook al zijn OHRB tot nu toe nog maar van een gelimiteerd aantal locaties geïsoleerd, blijkt er volgens kweek-onafhankelijke moleculaire analyses geen beperking te zijn voor hun geografische verspreiding. De belangrijkste omgevingsfactoren die de verspreiding en activiteit van de OHGB beïnvloeden worden besproken. Daarnaast worden de mogelijkheden om nieuwe OHGB te vinden in extreme milieus voorgesteld, aangezien de huidige OHGB isolaten bij neutrale pH's en lage zout concentraties overleven en groeien. Hieruit voortvloeiend is er in Hoofdstuk 7 de potentie voor het gebruik van organische halogeenverbindingen bekeken in de extreem zoute sedimenten van Lake Strawbridge, Australië. Dit resulteerde in de eerste beschrijving van de dechlorinering van chloroform en perchloroetheen (PCE) naar dichloromethaan en trichloroetheen uit een ongerepte alkalische en extreem zoute omgeving. In combinatie met de eerder door anderen beschreven productie van organische halogeenverbindingen op deze plek geeft Hoofdstuk 7 een interessant bewijs van de mogelijkheid van onafhankelijke biologische chlorinering en dechlorinering in halogeen- cycli die zich in extreem zoute meren afspelen.

De meeste moleculaire onderzoeken om de aanwezigheid en functionaliteit van OHGB aan te tonen, gebruiken een primer gebaseerde detectie van $16 \mathrm{~S}$ ribosomaal RNA- en reductief dehalogenase (RDase) coderende genen. Een uitgebreid overzicht van de specifieke primers 
voor RDase coderende genen en de toepassing van primer-afhankelijke technieken wordt gegeven in Hoofdstuk 3. Daarnaast bespreken we de huidige trend in het gebruik van primeronafhankelijke technieken om onze kennis over OHGB en hun RDase genen te vergroten. Door de voor- en nadelen van primer-afhankelijke en -onafhankelijke methodes is het beter om een combinatie van beide te gebruiken.

Dehalobacter restrictus PER-K23 $3^{\mathrm{T}}$ is gebruikt in Hoofdstuk 4 als model organisme om corrinoïde auxotrofie te bestuderen. Corrinoïdes zijn essentiële co-factoren van RDase enzymen. Vijftig $\mu \mathrm{g} / \mathrm{L}$ corrinoïde is de essentiële hoeveelheid voor het dechloreren van PCE. Dit kon worden bevestigd door transcriptie en proteoom analyses. Genoom analyse heeft verder uitgewezen dat de niet functionele cobalamine biosynthese in D. restrictus wordt veroorzaakt door een verkorting van $101 \mathrm{bp}$ in het $c b i H$ gen. Dit suggereert een verhoogde regulatie van cobalamine opname en transport systemen, om aan de corrinoïde behoefte te kunnen voldoen.

Het belang van syntrofe interacties voor goede groei van OHGB staat in de Hoofdstukken 5 en 6 beschreven. In Hoofdstuk 5 zijn dit bacteriën die behoren tot het Bacteroidetes phylum, de Clostridiales orde en sulfaat reduceerders uit de Deltaproteobacteria klasse. Deze bacteriën zouden elektronen en/of cofactoren kunnen bieden aan OHGB die chloor-benzeen kunnen gebruiken, zoals Dehalococcoides mccartyi en Dehalobacter. De bacteriën werden gevonden in ophopingen die werden verkregen van verontreinigd rivier- en havensedimenten. In Hoofdstuk 6 is een vergelijkbare syntrofe interactie geobserveerd tussen D. mccartyi en fermenterende en/of sulfaat reducerende bacteriën. De syntrofie tussen deze bacteriën bestond uit een robuuste dechlorering van gechloreerde ethenen welke ontstond na in situ biostimulatie door middel van glycerol injectie. Daarnaast dienen bacteriën behorend bij de Epsilonproteobacteria en Deferribacteres, welke in een laat stadium van de studie zijn gaan groeien, mogelijk als detoxificeerders van sulfide en stimuleren daarmee D. mccartyi. Echter deze bacteriën zouden mogelijk ook inhibeerders kunnen zijn en verschijnen na de belangrijkere syntrofen die de feitelijke fermentatie en sulfaat reductie hebben gedaan.

Concluderend, verbreedt deze thesis ons begrip binnen de ecofysiologie en verspreiding van OHGB, waarbij hun aanwezigheid is gevonden in de meeste milieus. Daarnaast geven we meer bewijs voor hun afhankelijkheid van nutriënten van andere microben in het ecosysteem. Daarmee vergroot dit onderzoek onze kennis over de anaërobe microbiële afbraak van 
organische halogeneenverbindingen, en kunnen zo strategieën voor bioremediatie worden geëvalueerd en verder worden verbeterd. 


\section{Acknowledgments}

Four years is not a short time period, and it was not easy to make the decision to leave my family half a world away and pursue my own dream here as a $\mathrm{PhD}$ candidate, especially for a person who had never ever leave her own country before. But I made it! And we made it! The past four years are beautiful time and so much great memories we created together. I would like to take this valuable opportunity to express my sincere appreciation for all the great persons I met, to thank you for the countless help and support.

Hauke, thank you very much for giving me the chance to work with you as a $\mathrm{PhD}$ student. You can't image how happy I was when you said ' yes' in our first Skype meeting and invited me to join your group. You are a great scientist and a respectable person, and it is highly appreciated for your patience, support and guidance. I don't remember how many times I am impressed by your profound knowledge and your passion into science. And you always give me freedom, let me think by myself and encourage me to be an independent and creative scientist. Thank you for all the mid-night emails, all the inspiring discussions and everything you have done for me.

Siavash, it is a great pleasure to work with you. I am super lucky to have you as my daily supervisor and get to know you as a good friend. You are dedicated in science, and you are super organized. It is great to have you in the supervision team, who always believe in me, push me forward and keep me on the right track. Thanks for being available whenever I dropped by for questions, and fast responding to emails and whatever I needed. Thanks for backing me up and helping me going through all the difficult time in my $\mathrm{PhD}$. When my first co-culture project failed, when I was depressed with writing for book chapter and called you, when I was worried about completing the thesis on time, it was you being there to lead the project with tons of good ideas and to encourage me to be confident again with my $\mathrm{PhD}$. I certainly owe you a lot, and for sure I couldn't finish my $\mathrm{PhD}$ in four years if without your help. Thanks for all the professionally and personally guides.

I would like to thanks all the external collaborators for your valuable contribution to this thesis. Aamani, Julien and Christof, thanks for inviting me joining the cool project of Dehalobacter restrictus, it was a valuable opportunity especially considering the failure of my own Dehalobacter project. Sjef, thanks for your technical guide on proteomics. Laura, thanks for your inputs and comments on the book chapter. Pieter, Steffi, Danuta and Dirk, your contribution made the chlorobenzene story possible, I appreciate that. Edoardo, Maria, 
Heinrich, Martin, Dirk and Winnie, thanks for your inputs on the field study. Alexander and Andreas, the collabration with you on the hypersaline story was a great experience.

I am glad to join the MOLECO family. First of all, special acknowledgment to our dehalo team; it was nice to have our monthly Dehalo-meeting for fruitful discussion. Farai, thanks to be my daily supervisor in my first year of $\mathrm{PhD}$. I am also grateful that you showed me how to prepare anaerobic media and the tips to work with the co-culture. Thomas, thanks to teach me all the molecular techniques and I certainly learned a lot from you. As you said, I can be counted as ' 0.5 ' student of you. Ying, thanks for taking care of my experiment when I went back China for holidays, and you are the best roommate ever! Peng, thanks for taking over the hypersaline sediment project when I was stuck in front of the computer to write $\mathrm{PhD}$ thesis. Also Tian, Gilbert and Tom Bosma deserve thanks. The weekly MOLECO meeting is a great chance to communicate with the colleagues and you always surprise me with your amazing ideas and suggestions, therefore, I would like to thank all the MOLECO people. And I will never forget all the great time we had during the lunch breaks, MOLECO cake time in the secret corner/rabbit garden and the lovely Christmas dinners. Last but not least, I would like to thank Javier for your support on the bioinformatics analysis, which helped to build up three scientific chapters in this thesis. And Detmer, thanks for the nice discussions and your input in the hypersaline story.

I would like to take this chance to thank all the MICPHYS people for your help, since I did most of my anaerobic works in MICPHYS lab for the past four years and every now and then, there is an idea coming to my mind that I belong to you instead MOLECO;-) Fons, thanks for allowing me working in the MICPHYS lab and your contribution in the field study is highly appreciated. Special thanks to Ton for the excellent technical assistance on anaerobic tent and all the HPLC and GCs, and thanks for your patience to answer all my stupid questions. Monika, everyone agrees that you are doing a great job as a technician, thanks for your guide in the lab.

Willem, thanks for arranging the $\mathrm{PhD}$ meeting and all the comments/suggestions on my presentations. Anja, thanks for your help on countless paper works, and you are always nice to me whenever I go to the secretary room, I really appreciate that. Carolien, thanks for helping me out when Anja is not around, and good luck with your new job. Wim, if without you I don't know how the whole MIB department can operate, thanks for taking care of the PCs and arranging the table for my student. Ineke, you are always enthusiastic and willing to 
help, thanks for ordering lab materials for me. Philippe, I really like the lab tour you gave, and thank you for giving me second chance when I failed the examination on security quizzes. Also thanks to Sjon, Hans, Jorn, Steven and Tom for your technical assistance.

I would like to give special acknowledgment to my officemates. Jing, you don't know how grateful I was to have you in the same office and being a close friend. You were already supper helpful even before my arrival here. You helped me to quickly adapted to the new environment and introduced me to all your friends. I will never forget your kindness. Floor, thanks for taking care of all my Dutch paper works including the Dutch summary in this thesis, and many times you helped me to call the Dutch company for the bills and NS card. My life in The Netherlands is much easier to have you around. And thanks for giving me the bouquet in your wedding and being my paranymph. Mauricio, you brought a lot of laugh to the office and I will miss our fights on the heating... All the office dinners we had with you guys and Tommer and Fernanda, those were great time, I really enjoyed. Alex (U.), even though you joined later, you were a great friend to talk with, scientific discussion or small chats, and you always bring fresh ideas to my life. Your passion on fishing, archery and 'choking' people down, I think I will never meet a same person like you. Thanks for making that nice heatmap for the hypersaline lake chapter and I am really happy that you can be my paranymph. Joan, I miss your pancakes! And your help on editing the propositions and final checking the whole thesis book is highly appreciated. Angela, Alessandra and Ioannis (K.), even though it was only for a short time period, I am really happy to know you and to share the same office. Thanks to LooWee, Johanna, Jie, Sudarshan and Dat, my new officemates in Helix.

The PhD trip to USA and Canada in 2013 was a great memory. All the credits go to the PhD trip committee, thanks for the organization. Thanks Stam, who was our leader. Corina, Laura and Melvin, thanks for being our driver. Janneke, orchestra concert in Boston was impressive, thanks for the planning. Sebastiaan, thanks for organizing the trip to New York. Jueeli, Teresita, Monir, Teunke and Kal, it was glad to know you more in person during the trip and we had a lot of fun together. Susakul, Nam and Jing, it was a nice experience to share the same hotel room with you, and I will never forget all those happy night talks we had together.

Dennis and Siavash, we had nice time in FEMS conference 2013 in Leipzig, Germany. Thanks for your accompany. 
Benoit, Monika and Tijn, it was amazing time that we spent together to organize the lab trip in 2014. The wine tasting in cellar and museum, visiting Historische kelders in Anrhem, playing games in Avontuurfabriek and end up with the dinner in hof van wageningen, I think we did pretty good job!

Irene, Peer, Teresita and Javier, our trip to Seoul, South Korea for the ISME conference 2014 was great. I will never forget all the lovely food (e.g. spicy chicken feet) and sauna bath we tried together besides the great scientific programs in the conference.

Alex (K.), Nico, Kees, Anna, Jasper (S.), Maarten and Ruben, it was great experience to work with you in the commetee for $\mathrm{PhD}$ trip 2015. Alex (K.), our chairman, your leadership made us being a team. Jasper (S.) and Kees, thanks for taking care of the sponsors. Anna and Maarten, really cool scientific program you made for the trip. Ruben, working with you to find the best/cheapest flights, hotels and car renting companies in western USA was a lot of fun. Last but not least, Nico, thanks for taking over all the rest works, you are a superman.

The best car pool ever, Detmer, Romy, Alex (U.), Ying and LooWee, I was so lucky to stay in the same car with you and our trip from Santa Cruz to Los Angeles was the highlight of the whole journey. Thanks for creating such a good atmosphere and all the funny chats.

A two-week journey with another 11 people from 11 different countries was definitely a unique experience. Our travelling from San Diego all the way to Yellowstone was amazing. Thanks to Michael, Johanna, Alex (U.), LooWee, Lara, Klaudyna, Ying, Alex (K.), Nico, Anna and Ioannis (M.), that went without a big fight and thank God, we are still friends.

Yolande and Jan, we had lots of fun, the mahjong nights, the tour to Zuiderzee, and those nice foods we tried together with my parents. It was great to know you in person.

Katija, it was nice experience to be paranymph with you for Floor's defence.

Thanks to Ying, Siavash, Marcelle and Indra, supervising BSc/MSc practical courses with you was a lot of fun. Thanks to Serve and Carolien for inviting us to join your courses.

Anna, Brenda and Celia, our small team 'Girls up and out' turned out not being that successful in 'Steptember' acitivity 2015. But we had fun together and we contributed to support treatment for cerebral palsy, which is meaningful. 
Thanks to Alex (U.), Gerben, Ioannis (K.), Hanne, LooWee, Klaudyna and Javier to make our 'Molecutes' team composition in We day 2016, and thanks to Nikolas who joined us in the last minute. Even though we got totally wet in the first game and went to speed dating immediately, our team was in the top 5 in the end. Good job!

Our small squash playing group, Denny, Peer, Sudarshan, LooWee and Lara, playing with you was a great release for me. Thanks for the competitions.

I don't remember since when we have started organizing the girl's dinners. Thanks to Teresita, Catalina, Ying, Anna, Jing, Nam, Irene, Cristina, Yuan, Jueeli, Brenda, Tika, Monika, Maria, Lara, Rebecca, Susakul, Irene, Dorett and Diana, certainly every time we all enjoyed a lot of the home-made foods from different countries.

I would like thank my Chinese friends who are not mentioned above. YiFan, Jie, Yue Han and Prof. Hao, it was nice to get to know you in the lab. Thanks to JunCai Chen, YuanYuan Zhang, Fang Gou, YiQian Fu, Yin Song, HuChen Li, GuoZhi Bi, LiHe Xu, Peng Wang, YongFu Tang \& JiaCui, we came to WUR more or less the same time and we are going through the same stages in our $\mathrm{PhD}$ studies, so it is great to have you around.

Jackson Yi, you are much younger than me, but I certainly learned a lot from you. Looking at you chasing your own dream and being a fabulous person have encouraged me to keep moving forward. Especially in the last period of my $\mathrm{PhD}$, it was dark time with endless writing and you light up my life. Stay in Asteroid B-612, and be the little prince.

Last but no least, I am enormously grateful to my family! 首先我要感谢给了我生命的爸爸 妈妈, 谢谢你们一路以来所给予的无限关爱和支持。我今天所获得的一切都离不开你 们的辛勤付出和言传身教。如果没有你们的鼓励和鞭策, 我应该不会鼓起巨大的勇气 来到离家这么远的地方追求自己的梦想。还有亲爱的爷爷奶奶, 谢谢你们一手带大了 我, 会在天上笑着祝福我吧。还要感谢所有的家人们, 谢谢叔叔, 阿姨, 姑姑, 阳 叔, 满满和小尤阿姨的爱护, 谢谢外婆, 外公, 大姨和舅舅的关心, 还有谢谢鲁婧, 欧阳泽斌, 黄秋实, 楚佳恒和李瑜的陪伴! 我很庆幸出生在这么有爱的大家庭里, 谢 谢你们, 我爱你们! 


\section{About the author}

Yue Lu was born on April 9th, 1989, in Shaoshan, Hunan province, China. In 2006, she started her bachelor study at the Department of Resources and Environmental Science, China Agricultural University (CAU), Beijing. In 2009, she started her BSc thesis entitled "Transcriptional response of methanogenic community to oxygen exposure in rice field soil", under the supervision of Prof. Dr. Yahai Lu in the Lab of Molecular Ecology-Max Planck Partner Group. In 2010, she obtained her BSc degree of Science and received the award of Outstanding Undergraduate in Beijing. Inspired by the great experience of her stay in the lab of Prof. Dr. Yahai Lu, she continued with her MSc studies in the same year and completed her MSc thesis entitled "Effect of temperature on the structure and activity of a methanogenic archaeal community during rice straw decomposition", which resulted in a first-author publication in the journal of Soil Biology and Biochemistry. After obtaining her MSc degree in 2012, she moved to the Netherlands and started a PhD project with the scholarship from China Scholarship Council at the Laboratory of Microbiology, Wageningen University, under the supervision of Prof. Dr. Hauke Smidt and Dr. Siavash Atashgahi.

During her study time, Yue Lu has been actively involved in diverse social activities. She joined the Student Union in Department of Resources and Environmental Sciences in CAU for two years, and she served as a volunteer in 29th Olympic Games, Beijing and in China Environmental Culture Promotion Association (member of the Ministry of Environmental Protection of China). 


\section{List of Publications}

Yue Lu, Li Fu, Yahai Lu, Floor Hugenholtz, Ke Ma (2015). Effect of temperature on the structure and activity of a methanogenic archaeal community during rice straw decomposition. Soil Biology and Biochemistry 81: 17-27. doi: 10.1016/j.soilbio.2014.10.031

Aamani Rupakula*, Yue Lu*, Thomas Kruse, Sjef Boeren, Christof Holliger, Hauke Smidt, Julien Maillard (2015). Functional genomics of corrinoid starvation in the organohaliderespiring bacterium Dehalobacter restrictus strain PER-K23. Frontiers in Microbiology 5:751. doi: 10.3389/fmicb.2014.00751.

Yue Lu*, Siavash Atashgahi*, Laura A. Hug, Hauke Smidt (2015). Primer that target functional genes of organohalide-respiring bacteria. In: T.J. McGenity et al. (eds.) Hydrocarbon and Lipid Microbiology Protocols, Springer Protocols Handbooks. Springer. doi: 10.1007/8623_2015_75

Siavash Atashgahi*, Yue Lu*, Hauke Smidt (2016). Overview of known organohalide respiring bacteria - phylogenetic diversity and environmental distribution. In: L. Adrian, F.E. Löffler (eds.) Organohalide Respiring Bacteria. Springer.

Yue Lu, Javier Ramiro-Garcia, Pieter Vandermeeren, Steffi Herrmann, Danuta Cichocka, Dirk Springael, Siavash Atashgahi, Hauke Smidt (2016). Dehalogenation of three tetrachlorobenzenes isomers by contaminated harbor sludge-derived enrichment cultures follows thermodynamically favorable reactions. Under review.

Siavash Atashgahi, Yue Lu, Ying Zheng, Edoardo Saccenti, Maria Suarez Diaz, Javier Ramiro-Garcia, Heinrich Eisenmann, Martin Elsner, Alfons JM Stams, Dirk Springael, Winnie Dejonghe, Hauke Smidt (2016). Geochemical and microbial analysis of groundwater at a site with biostimulated reductive dechlorination. Under review.

Siavash Atashgahi, Yue Lu*, Javier Ramiro-Garcia*, Peng Peng, Farai Maphosa, Detmer Sipkema, Winnie Dejonghe, Hauke Smidt, Dirk Springael (2016). Geochemical parameters and reductive dechlorination determine cometabolic vs metabolic aerobic VC biodegradation at oxic/anoxic interface of hyporheic zones. Submitted for publication. 
Yue Lu*, Peng Peng* ${ }^{*}$ Alexander Martin Ruecker, Javier Ramiro-Garcia, Alexandr Umanetc, Andreas Kappler, Detmer Sipkema, Siavash Atashgahi, Hauke Smidt (2016). Organohalide respiration in pristine hypersaline lakes. Manuscript in preparation.

Ying Zheng, Yue Lu, Maria Suarez Diaz, Alfons JM Stams, Siavash Atashgahi, Hauke Smidt (2016). Anaerobic biodegradation of long-chain fatty acid methyl esters by an oleatedegrading enrichment culture. Manuscript in preparation.

Peng Peng, Jasper Koehorst, Yue Lu, Detmer Sipkema, Jaap S. Sinninghe Damste, Alfons JM Stams, Siavash Atashgahi, Hauke Smidt (2016). Isolation, identification and genomic characterization of an organohalide-respiring Desulfoluna bacterium. Manuscript in preparation.

*These authors contributed equally to this work 


\section{Co-author affiliations}

Siavash Atashgahi 1,2,3

Sjef Boeren ${ }^{4}$

Danuta Cichocka ${ }^{3}$

Jaap S. Sinninghe Damste ${ }^{5,6}$

Winnie Dejonghe ${ }^{2}$

Maria Suarez Diaz ${ }^{1}$

Heinrich Eisenmann ${ }^{7}$

Martin Elsner ${ }^{8}$

$\mathrm{Li} \mathrm{Fu}^{9}$

Steffi Herrmann ${ }^{3}$

Christof Holliger ${ }^{10}$

Laura A. Hug ${ }^{11}$

Floor Hugenholtz ${ }^{1,12,13}$

Andreas Kappler 12, 14

Jasper Koehorst ${ }^{15}$

Thomas Kruse ${ }^{1}$

Yahai $\mathrm{Lu}^{9}$

$\mathrm{Ke} \mathrm{Ma}^{9}$

Julien Maillard ${ }^{10}$

Farai Maphosa ${ }^{1}$

Peng Peng ${ }^{1}$

Javier Ramiro-Garcia ${ }^{1,15}$

Alexander Martin Ruecker ${ }^{16,17}$

Aamani Rupakula ${ }^{10}$

Edoardo Saccenti ${ }^{15}$

Detmer Sipkema $^{1}$

Hauke Smidt ${ }^{1}$

Dirk Springael $^{3}$

Alfons JM Stams ${ }^{1}$

Alexandr Umanetc ${ }^{1}$

Pieter Vandermeeren ${ }^{3}$

Ying Zheng ${ }^{1}$

${ }^{1}$ Laboratory of Microbiology, Wageningen University, The Netherlands

${ }^{2}$ Separation and Conversion Technology, Flemish Institute for Technological Research (VITO), Belgium

${ }^{3}$ Division of Soil and Water Management, KU Leuven, Belgium

${ }^{4}$ Laboratory of Biochemistry, Wageningen University, The Netherlands

${ }^{5}$ Department of Marine Organic Biogeochemistry, NIOZ Royal Netherlands Institute for Sea Research, The Netherlands 
${ }^{6}$ Department of Earth Sciences, Utrecht University, The Netherlands

${ }^{7}$ Isodetect GmbH, Germany

${ }^{8}$ Institute of Groundwater Ecology, Helmholtz Zentrum München-National Research Center for Environmental Health, Germany

${ }^{9}$ College of Resources and Environmental Sciences, China Agricultural University, China

${ }^{10}$ Laboratory for Environmental Biotechnology, Ecole Polytechnique Fédérale de Lausanne, Switzerland

${ }^{11}$ Department of Earth and Planetary Science, University of California, Berkeley, USA

${ }^{12}$ Netherlands Consortium for Systems Biology, The Netherlands

${ }^{13}$ TI Food and Nutrition, The Netherlands

${ }^{14}$ Center for Geomicrobiology, Department of Bioscience, Aarhus University, Denmark

${ }^{15}$ Laboratory of Systems and Synthetic Biology, Wageningen University, The Netherlands

${ }^{16}$ Geomicrobiology, Center for Applied Geosciences, University of Tuebingen, Germany

${ }^{17}$ Biogeochemistry-Baruch Institute of Coastal Ecology and Forest Science, Clemson University, USA 


\section{SENSE}

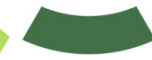

Netherlands Research School for the

Socio-Economic and Natural Sciences of the Environment

\section{I P L O M A}

For specialised PhD training

The Netherlands Research School for the

Socio-Economic and Natural Sciences of the Environment

(SENSE) declares that

\section{Yue Lu}

born on 9 April 1989 in Hunan, China

has successfully fulfilled all requirements of the Educational Programme of SENSE.

Wageningen, 13 September 2016

the Chairman of the SENSE board

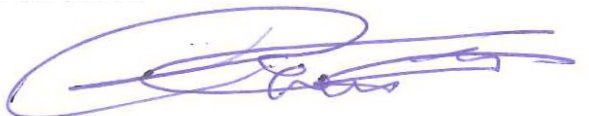

Prof. dr. Huub Rijnaarts the SENSE Director of Education

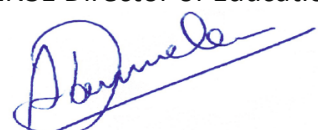

Dr. Ad van Dommelen

The SENSE Research School has been accredited by the Royal Netherlands Academy of Arts and Sciences (KNAW)

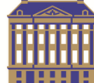




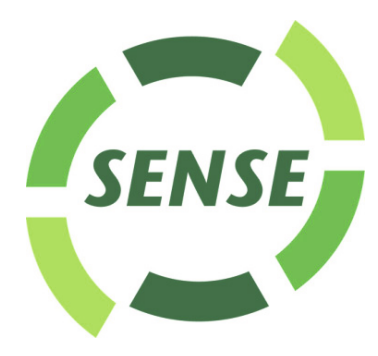

The SENSE Research School declares that Ms Yue Lu has successfully fulfilled all requirements of the Educational PhD Programme of SENSE with a work load of 37.7 EC, including the following activities:

\section{SENSE PhD Courses}

- Environmental research in context (2013)

o Research in context activity: 'Co-organising PhD trip 2015 to Californian research institutes', Laboratory of Microbiology and Laboratory of Systems and Synthetic Biology of Wageningen University (2015)

\section{Other PhD and Advanced MSc Courses}

o Information literacy including EndNote, Wageningen University (2012)

- Systems biology: statistical analysis of omics data, Wageningen University (2012)

- Hydrogen sulphide gas (H2S) course, Netherlands Safety Education BV (2013)

- Techniques for writing and presenting scientific papers, Wageningen University (2013)

- Interpersonal communication for PhD students, Wageningen University (2013)

- Metagenomics approaches and data analysis, Netherlands Bioinformatics Centre (NBIC) (2013)

- Basic training in software packages ARB and SILVA, Wageningen University (2014)

\section{Management and Didactic Skills Training}

- Teaching practical course 'Microbial Physiology', Wageningen University (2013)

- Supervising MSc student with thesis entitled 'Enrichment of chlorobenzene degrading cultures', Wageningen University (2014)

- Teaching practical course 'Research Methods Microbiology', Wageningen University (2015-2016)

\section{Oral Presentations}

- Functional genomics of Dehalobacter sp. E1 in syntrophic interaction with Sedimentibacter sp. B4. FOR 1530 Research Unit Conference - Friedrich Schiller University Jena, 19-20 July 2013, Leipzig, Germany

- Chasing organohalide respirng bacteria (OHRB) from pristine hypersaline lakes. FOR 1530 Research Unit Meeting - Friedrich Schiller University Jena, 14-15 April 2016, Weimar, Germany

\section{SENSE Coordinator PhD Education}


The work of Yue Lu was performed at the Laboratory of Microbiology, Wageningen University, The Netherlands, and financially supported by a grant of BE-Basic-FES Funds from the Dutch Ministry of Economic Affairs. Yue Lu was sponsored by a CSC Fellowship.

Cover photo: DingSheng Li (李定胜)

Thesis layout: Yue Lu

Printed by: GVO drukkers \& vormgevers, Ede, The Netherlands

Financial support from the Laboratory of Microbiology, Wageningen University, for printing of this thesis is gratefully acknowledged 
